\title{
Versuche über Längsschub und Querbiegung in Druckplatten von Betonträgern
}

\section{Working Paper}

Author(s):

Badawy, Maher; Bachmann, Hugo

Publication date:

1977

Permanent link:

https://doi.org/10.3929/ethz-a-000124642

Rights / license:

In Copyright - Non-Commercial Use Permitted

Originally published in:

Bericht / Institut für Baustatik und Konstruktion ETH Zürich 6504(8) 
Versuche über Längsschub und Querbiegung in Druckplatten von Betonträgern

Maher Badawy

Hugo Bachmann 


\title{
Versuche über Längsschub und Querbiegung in Druckplatten von Betonträgern
}

\author{
von \\ Dr. sc. techn. M. Badawy \\ Prof. Dr. H. Bachmann \\ Institut für Baustatik und Konstruktion \\ Eidgenőssische Technische Hochschule Zürich
}


1. Einleitung

1.1 Allgemeines

1.2 Zielsetzung

1.3 Bemessungsmodelle

1.3.1 Längsschub allein

1.3.2 Längsschub mit Querbiegung

1.4 Versuchsplanung

1.4.1 Konzeption der Versuchsträger

1.4.2 Parameteranalyse

2. Versuchsbalken

2.1 Beschreibung

2.1.1 Abmessungen, Armierungen

2.2 Baustoffe

2.2.1 Armierungsstahl

2.2 .2 Beton

2.3 Bemessung und rechnerische werte 9

2.3.1 Berechnete Lasten 9

2.3.2 Bemessung der Stegarmierung 10

2.3.3 Bemessung der Plattenarmierung 11

2.3.4 Konstruktive Ergänzungen der Modellarmierungen 11

$\begin{array}{ll}\text { 3. Versuchsdurchführung } & 13\end{array}$

3.1 Versuchsanlage $\quad 13$

3.2 Laststufen und Versuchsablauf 13

$\begin{array}{ll}3.3 \text { Messungen } & 15\end{array}$

3.3.1 Lasten 15

3.3.2 Verformungen $\quad 15$

3.3.3 Risse 16

$\begin{array}{lr}\text { 4. Versuchsresultate } & 17\end{array}$

$\begin{array}{ll}4.1 \text { Allgemeines Tragverhalten } & 17\end{array}$

4.2 Traglasten 18

$\begin{array}{ll}4.3 \text { Plattenfliesslasten } & 18\end{array}$

$\begin{array}{lr}4.4 \text { Verformungen } & 19\end{array}$

4.4.1 Allgemeines $\quad 19$

4.4.2 Dehnungen der Stegarmierung 20

4.4.3 Durchbiegungen des Steges und der Platte. 20

4.4.4 Betonstauchungen in der Platte 20

4.4.5 Dehnungen der Plattenarmierung 21

4.5 Kräfte in der Plattenarmierung 23 
4.6 Risseverhalten $\quad 23$

$\begin{array}{ll}4.7 & \text { Bruchverhalten }\end{array} 25$

5. Vergleiche zwischen Versuchsergebnissen und Modellen 26

5.1 Längsschub allein (Balken Q1 und Q2) 26

5.2 Längsschub mit Querbiegung (Balken Q3, Q4, Q5) 27

6. Ergänzungen 29

6.1 Interpretation der Versuchsergebnisse 29

6.1 .1 Ausbreitwinkel 29

6.1 .2 Interaktion Biegung - Längsschub 29

6.2 Obere Schubspannungsgrenze 29

7. Folgerungen und vorläufige Empfehlungen 31

ZUSAMMENFASSUNG

RESUME

$\begin{array}{ll}\text { SUMMARY } & 37\end{array}$

$\begin{array}{ll}\text { VERDANKUNGEN } & 39\end{array}$

$\begin{array}{lr}\text { LITERATURVERZEICHNIS } & 40\end{array}$

$\begin{array}{ll}\text { BEZEICHNUNGEN } & 41\end{array}$

$\begin{array}{ll}\text { TABELLEN 1-8 } & 44\end{array}$

$\begin{array}{lll}\text { BILDER } & 1-66 & 49\end{array}$

$\begin{array}{lr}\text { ANHANG } & 120\end{array}$ 
Bei dünnwandigen Plattenbalken- und Hohlkastenquerschnitten aus Stahlbeton ist die Bemessung der Druckplatten im Anschlussbereich zu den Längsstegen ein noch ungelöstes Problem. Betrachtet man als einfaches Beispiel ein. Element des Trägers gemäss Bild 1, so können in einem Schnitt I-I je nach der äusseren Belastung folgende Schnittgrässen auftreten:

\section{a) Längsschub allein}

Wirkt eine Belastung $P$ in der Symmetrieebene des T-Trägers, so entsteht eine Längsschubkraft $\tau_{y x} \cdot d$.

\section{b) Längsschub mit Querbiegung}

Wirkt ferner eine Belastung $\bar{P}$ ausserhalb des Steges, so entsteht zum Längsschub hinzu ein Querbiegemoment $M_{q}$. (Die ebenfalls auftretende vertikale Schubkraft $\tau_{y x} \cdot d$ wird in der vorliegenden Untersuchung vernachlässigt.)

\section{c) Längsschub mit Querbiegung und Quervorspannung}

Ist die Platte in Querrichtung noch vorgespannt, so wirkt nebst Längsschub und Querbiegung zusätzlich noch die Vorspannkraft $V$.

Die Bemessung des Anschlussbereiches Platte - Steg bzw. die Berechnung der notwendigen Armierungen kann nach verschiedenen Modellen erfolgen. Diese führen indessen zu sehr unterschiedlichen und widersprüchlichen Resultaten. Daher wurden in den Jahren 1974 und 1975 am Institut für Baustatik und Konstruktion der ETH Zürich Versuche an insgesamt sieben Betonträgern durchgeführt (Bild 10). An zwei Trägern wurde das Problem "Längsschub allein" erforscht (Bild 18). Bei drei Trägern wurde zusätzlich eine Querbiegung aufgebracht (Bild 19). Die Ergebnisse dieser Versuche bildeten die Basis für die Prüfung von zwei weiteren Trägern, die in der Druckplatte die Beanspruchung "Längsschub mit Querbiegung und teilweiser Quervorspannung" aufwiesen.

Im vorliegenden Bericht wird über die Versuche "Längsschub allein" sowie "Längsschub mit Querbiegung" berichtet. Die Versuche mit Quervorspannung werden in einem separaten Bericht, [2], behandelt.

\subsection{Zielsetzung}

Das Ziel der hier beschriebenen Versuche war die Erforschung des Verformungs- und Tragverhaltens von Druckplatten in Betonträgern unter Längsschub sowie unter Längsschub und Querbiegung. Hierbei sollten verschiedene Bemessungsmodelle überprüft und anhand der Versuchsresultate verglichen werden. 
1.3 Bemessungsmodel le

Die wichtigsten Modelle, nach denen man die Beanspruchungen bzw. die Armierung im Anschlussbereich Platte - Steg bei Längsschub sowie bei Längsschub mit Querbiegung ermitteln kann, werden im folgenden zusammenfassend erklärt und diskutiert. Dies geschieht am Beispiel eines symmetrischen einfach gelagerten Plattenbalkens, der den durchgeführten Versuchen entspricht.

\subsubsection{Längsschub allein}

\section{a) Klassisches Modell}

Im Falle von Längsschub werden in der Praxis die Beanspruchungen im Anschlussbereich normalerweise nach dem klassischen Modell (Einfache Balkentheorie, Hauptzugspannung genl ermittelt, das in Bild 2 dargestellt ist. Die Normalspannung in der PlattenMittelebene $\sigma_{x}$ und die mittlere Schubspannung im Anschlussbereich $\tau_{y x}$ werden berechnet und daraus die Hauptzugspannung $\sigma_{h z}$ bestimmt. Betrachtet man ein Sägeschnittelement mit der Länge ds, dann wirkt an der Seite mit der Hauptzugspannung $\sigma_{h z}{ }^{\circ} d i e Z^{\prime}{ }^{-}$ kraft $z=\sigma_{h z} \cdot d \cdot d s \cdot \cos \varphi$. Normalerweise wird die Armierung nicht in der Richtung der Hauptzugspannung verlegt, sondern senkrecht zur Trägerachse. Ersetzt man nun die Kraft $Z$ durch Kräfte in der Armierungs- und Risserichtung (den zwei tragfähigen Richtungen\}, dann wirkt im Anschlussbereich die Zugkraft

$$
z_{B}=\sigma_{h z} \cdot d \cdot d s \quad \text { pro Länge ds }
$$

bzw. $\quad S=\frac{Z_{B}}{d s}=\sigma_{h z} \cdot d \quad$ pro Längeneinheit.

$Z_{B}$ muss von einer Plattenquerarmierung aufgenommen werden, die normalerweise je zur Hälfte oben und unten in die Platte eingelegt wird. Da die Zugkraft $S$ proportional zur Hauptzugspannung $\sigma_{h z}$ ist, wird die Verteilung der Plattenquerarmierung über die Balkenlänge affin zur $\sigma_{h z}$-Verteilung sein (Bild 2). Dies bedeutet viel Armierung in der Platte im Auflagerbereich und wenig, bzw. keine Armierung im mittleren Bereich.

Gegen dieses Modell können folgende Einwände vorgebracht werden (Bild 3 ):

Die aus der Längsbelastung $P$ nach klassischer Theorie an den zwei Plattenteilen 1 und 2 wirkenden Spannungen bzw. Kräfte sind in Bild 3a eingezeichnet. Würden diese Spannungen bzw. Kräfte tatsächlich auftreten, so wären die zwei Scheiben 1 und 2 im Gleichgewicht. In Wirklichkeit kann aber an der Stirnfläche des Plattenbalkens keine Schubspannung entstehen, da dort eine freie Oberfläche vorliegt. (Allenfalls könnte ein Querträger diese Aufgabe übernehmen; er würde dann auf Zug beansprucht.) Dadurch wird das Gleichgewicht der Scheibe 1 gestört $(\Sigma Y \neq 0, \Sigma M \neq 0)$. Ausserdem zeigt sich entlang des Lastquerschnittes $A-A$ eine Diskontinuität in der Schubspannung $\tau_{y x}$ : Links von A-A ergibt sich eine Schubspannung, rechts davon jedoch nicht. In Wirklichkeit werden die Spannungen links und rechts des Schnittes A-A jedoch entgegengesetzt gleich sein müssen, d.h. entlang $A-A$ sind entweder Schubspannungen oder keine Schubspannungen vorhanden.

Betrachtet sei vorerst der Fall, wo entlang A-A keine Schubspannungen wirken sollen (Bild 3b, Fall 1). Damit das Gleichgewicht der Scheibe 1 erhalten bleibt, können zwei Kräfte $C$ (Druck) und $Z$ ( $Z u g$ ) eingeführt werden. Im rechten Teil dieser Scheibe treten also zu den Längsschubspannungen auch noch Zugspannungen quer zur Balkenaxe auf. 
Wirken entlang A-A jedoch Schubspannungen, so entsteht in der Platte in diesem Schnitt eine Schubkraft T1 und eine Druckkraft D' (Bild 3b, Fall 2). Scheibe 2 ist erst dann im Gleichgewicht, wenn zusätzlich nebst einer Längsschubkraft $T$ " auch noch eine Zugkraft Z" eingeführt wird. Auch bei Scheibe 1 müssen zur Erhaltung des Gleichgewichtes beispielsweise eine Zugkraft $Z^{\prime}$ und eine Druckkraft $C^{\prime}$ eingeführt werden.

Aus diesen Betrachtungen geht deutlich hervor, dass im Anschlussbereich Platte - Steg nicht nur Schubspannungen, sondern zusätzliche Druck- und Zugkräfte wirken müssen. Diese treten in der Tat nicht konzentriert auf, sondern sie stellen die Resultierenden von Druck- bzw. Zugspannungen dar, welche über den Auflagerbereich bzw. mittleren Bereich der Platte verteilt sind. Der Verlauf dieser Spannungen wird von Verträglichkeitsbedingungen beeinflusst und kann daher auf einfache Weise nicht genauer angegeben werden. Fest steht aber, dass durch diese Normalspannungen, welche in der klassischen Theorie nicht berücksichtigt werden, das Bild der Hauptzugspannungen entlang der Platte verändert wird.

Bereits Mörsch hat in [1] darauf hingewiesen, dass im Anschlussbereich ausser Schubspannungen auch noch Druck- bzw. Zugkräfte entstehen müssen.

\section{b) Flanschfachwerk-Modell}

Besser geeignet als das klassische Modell erscheint ein Flanschfachwerk-Modell. Die Grundidee dieses Modells vermittelt Bild 4 (siehe auch [3]). Die horizontalen Kompnenten der Druckdiagonalenkräfte des Steges müssen sich in der Druckplatte ausbreiten. Dadurch entstehen Umlenkkräfte Z1, welche die Platte bzw. den Anschlussbereich Platte - Steg auf Zug beanspruchen. Nach Bild 4 beträgt die Zugkraft 21 jeweils $\frac{Q}{2} \cdot \cot \alpha \cdot \operatorname{tg} \beta$. Die Neigung der Stegdruckdiagonalen wird mit $\alpha=45^{\circ}$ angenommen ( $\alpha$ hat jedoch auf die total entstehende Querzugkraft keinen Einfluss). Der Ausbreitungswinkel $\beta$ lässt sich anhand Bild $5 \mathrm{zu} \beta=\operatorname{arc} \operatorname{tg} 0.5$ abschätzen. Somit ist im Flanschfachwerk die Zugkraft $Z 1=\frac{Q}{4}$. Die totale auftretende Zugkraft im Anschlussbereich (Bild 6) ergibt sich $z u \frac{Q}{4} \times$ Anzahl der Zugkräfte 21 , somit $Z_{\text {tot }}=\frac{Q}{4} \cdot \frac{a}{y}$. Dabei ist zu beachten, dass diese Zugkräfte $Z 1$ jeweils erst in einem Abstand b/2 vom Angriffspunkt der entsprechenden Stegdruckdiagonalen auftreten (Bild 6). Die erste Zugkraft tritt also bei $x=y+\frac{b}{2}$, die letzte bei $x=a+\frac{b}{2}$ auf. In Wirklichkeit wirken die Kräfte der Stegdruckdiagonalen nicht konzentriert wie in Bild 4, sondern verteilt, und wir nehmen an, dass sie über eine Länge (a-y) "verschmiert" in die Platte eingeleitet werden (Bild 6). Dementsprechend verteilen sich auch die im Anschlussbereich auftretenden Querzugkräfte über die Länge (a-y). Die pro Längeneinheit auftretende Zugkraft 22 beträgt somit

$$
Z 2=\frac{\text { totale Zugkraft }}{a-y}=\frac{Q}{4 y} \cdot \frac{a}{(a-y)} \text {. }
$$

Man kann dieses Modell verfeinern, indem der Einfluss der Stegbreite bo berücksichtigt wird: ein Teil der horizontalen Komponente der Stegdruckdiagonalen entsprechend der Plattenquerschnittsfläche über dem Steg (schraffierte Fläche rechts oben in Bild 6) wird sich nämlich nicht ausbreiten und verursacht somit keine Zugkräfte. Dadurch reduziert sich die im Anschlussbereich pro Längeneinheit auftretende Zugkraft auf $Z$, wobei

$$
z=z 2 \cdot \frac{b-b_{0}}{b}=\frac{Q}{4 y} \cdot \frac{a}{a-y} \cdot \frac{b-b_{o}}{b}
$$

Diese Zugkräfte $Z$ treten zwischen $x=y+\frac{b}{2}$ und $x=a+\frac{b}{2}$ auf und werden als massgebend für die Beanspruchung des Anschlussbereiches angesehen. 
Wie beim klassischen Modell kann die resultierende Plattenquerarmierung je zur Hälfte oben und unten in die Platte eingelegt werden.

\section{c) Vergleich Klassisches Modell - Flanschfachwerk-Modell}

Aus dem Flanschfachwerk-Modell ergibt sich, dass in der Platte im Bereich der Einzellast Zugkräfte entstehen, während der Auflagerbereich unbeansprucht bleibt (Bild 6). Diese Feststellung steht total im Widerspruch mit dem klassischen Modell: grösste Beanspruchung der Platte im Auflagerbereich und kleine bzw. keine Beanspruchung im mittleren Bereich (Bild 2).

Vergleicht man ferner die Höhe der Beanspruchungen nach den beiden Modellen, so ergibt sich folgendes: Das klassische Modell entspricht dort wo $\sigma_{x}=0$ ist, d.h. über dem Auflager, einer Kraftausbreitung unter $45^{\circ}$ (reiner Schubspannungszustand, $\sigma_{h z}=\tau$, $\operatorname{tg} \beta=1.0\}$. Die notwendige Armierung ist etwa doppelt so gross wie diejenige nach dem Fachwerk-Modell, die sich indessen im Balkeninnern ergibt. Beim Fachwerk-Modell ist die Querzugkraft unabhängig von der Grösse der Normalkraft im Druckflansch, während beim klassischen Modell $\sigma_{x} z u$ einer starken Abminderung der erforderlichen Armierung führt.

\subsubsection{Längsschub mit Querbiegung}

Für den Fall Längsschub mit Querbiegung kann die Plattenarmierung nach einem der folgenden drei Modelle bestimmt werden (Bild 7):

\section{a) Superposition der Armierungen aus Hauptzugspannungs-Modell und Querbiegung}

Die Armierung für Längsschub wird nach dem klassischen Modell (Bild 2) ermittelt und je zur Hälfte oben und unten in der Platte vorgesehen. Die Armierung für das Querbiegemoment $M_{q}$ wird nach der normalen Biegetheorie bestimmt und, in Anlehnung an die Norm SIA 162, Art. 3.12, derjenigen für Längsschub überlagert (Bild 7a). Es braucht also eine obere und eine untere Armierungslage in der Platte. Nach diesem Modell wird heute in der Praxis normalerweise bemessen.

\section{b) Superposition der Armierungen aus Flanschfachwerk-Modell und Querbiegung}

Die Armierung für Längsschub wird für die Kraft $Z$ gemäss Flanschfachwerk-Modell berechnet und wiederum je zur Hälfte der oberen und unteren Lage in der Platte zugewiesen. Die Armierung für das Querbiegemoment wird wie unter a) beschrieben bemessen. Beide Armierungen werden dann im Querschnitt superponiert (Bild 7b). Auch hier braucht es zwei Armierungslagen.

\section{c) Armierung für Querbiegung mit Querzugkraft aus Flanschfachwerk-Modell}

Die axiale Zugkraft $Z$ (aus Flanschfachwerk-Modell) und das Querbiegemoment $M_{q}$ werden in einer Resultierenden $N$ zusammengefasst, die am Querschnitt exzentrisch wirkt (Bild 7c). Die Armierung wird darauf nach den Regeln der Biegung mit Axialzugkraft ermittelt. Demgemäss wird im Querschnitt eine obere Armierung benötigt, während unten (sofern $N$ ausserhalb von $F_{\text {eo }}$ liegt) eine Druckkraft entsteht und somit keine rechnerische Armierung notwendig wird. 


\subsubsection{Konzeption der Versuchsträger}

Um das Verformungs - und Tragverhalten von Druckplatten im Anschlussbereich mit dem Steg abzuklären, und die im letzten Abschnitt beschriebenen Modelle für "Längsschub allein" sowie für "Längsschub mit Querbiegung" zu überprüfen, wurde ein Versuchsprogramm ausgearbeitet. Dieses sah die Prüfung von fünf Stahlbeton-Plattenbalken, Q1 bis Q5, vor. Die ersten zwei Balken, Q1 und Q2, wurden dem Problem "Längsschub allein" zugeordnet, die übrigen drei, Q3 bis Q5, dem Problem "Längsschub mit Querbiegung". Abmessungen. Betonqualität und Stegarmierung wurden bei allen fünf Balken gleich gehalten. Die Plattenarmierung dagegen wurde jeweils nach einem bestimmten Modell konzipiert und zwar:

Balken Q1: nach dem klassischen Modell (Hauptzugspannungsmodell)

Balken Q2: nach dem Flanschfachwerk-Modell

Balken Q3: nach dem Modell "Superposition der Armierungen aus HauptzugspannungsModell und Querbiegung"

Balken Q4: nach dem Modell "Superposition der Armierungen aus FlanschfachwerkModell und Querbiegung"

Balken Q5: nach dem Modell "Armierung für Querbiegung mit Querzugkraft aus Flanschfachwerk-Modell"

\subsubsection{Parameteranalyse}

Die Abmessungen der Balken und die Belastungsanordnung sollten so. gewählt werden, dass eine gegebene Belastung ( $P$ bzw. Q) möglichst grosse Hauptzugspannungen bzw. Zugspannungen infolge Längsschub im Anschlussbereich Platte - Steg hervorruft. Deshalb wird im folgenden die Abhängigkeit dieser Spannungen von den verschiedenen Balkenparametern untersucht.

\section{a) Aufgrund des Klassischen Modells}

Nach diesem Modell ist die Hauptzugspannung $\sigma_{h z}$ für die Beanspruchung des Anschlussbereiches massgebend. Sie ist am grössten beim Auflager, wo sie gleich gross ist wie die dort wirkende Schubspannung $\tau_{x y}$ (Bild 2). Beim Auflager gilt also:

$$
\sigma_{h z A}=\tau_{x y}=\frac{Q \cdot S_{i}}{I_{i} \cdot d}=\frac{Q \cdot\left(S_{i} / S_{i D}\right)}{\left(I_{i} / S_{i D}\right) \cdot d},
$$

wobei: $\quad S_{i} \quad$ statisches Moment des Plattenteils rechts vom Schnitt a-a bezüglich $n-n$ (Bild 8 )

$=\frac{b-b o}{2} \cdot d \cdot\left(x-\frac{d}{2}\right)$

$S_{i D} \quad=$ statisches Moment der Druckzone bezüglich $n-n$

$=b \cdot d\left(x-\frac{d}{2}\right)+b_{0}(x-d)\left(\frac{x-d}{2}\right)$

$I_{i} \quad=$ ideelles Trägheitsmoment des Querschnittes bezüglich $n-n$

$I_{i} / S_{i D}=y=$ Hebelarm der inneren $\operatorname{Kräfte}($ siehe [3]). 
Vernachlässigt man im Ausdruck für $S_{i D}$ den zweiten Summanden (Anteil der Druckzone unter der Platte, schraffierte Fläche in Bild 8), so ist die Hauptzugspannung

$$
\sigma_{h z A}=\tau_{x y}=\frac{Q}{y \cdot d} \cdot \frac{b-b a}{2 b}=\frac{Q}{2} \cdot \frac{1}{y \cdot d}\left(1-\frac{b}{b}\right)
$$

Diese Formel drückt die Abhängigkeit der Hauptzugspannung beim Auflager, $\sigma_{h z} A^{\text {, von }}$ den verschiedenen Parametern aus und zeigt, dass bei gegebener Querkraft $Q$ die Hauptzugspannung mit kleineren Werten von $y$, $d$ und $b_{0} / b$ zunimmt.

Wird ein Schnitt unmittelbar ausserhalb der Last betrachtet, so kann die dortige Hauptzugspannung näherungsweise wie folgt ausgedrückt werden (siehe [3]):

$$
\sigma_{h z} \simeq \tau_{x y}-\frac{\sigma_{x}}{3}
$$

Es gilt: $\quad \sigma_{x}=\frac{M}{y} \cdot \frac{1}{b \cdot d}=\frac{Q \cdot a}{y} \cdot \frac{1}{b \cdot d}$

Damit wird

$$
\sigma_{h z}=\frac{Q}{2} \cdot \frac{1}{y d}\left[\left(1-\frac{b o}{b}\right)-\frac{2}{3} \cdot \frac{a}{b}\right]
$$

Daraus ergibt sich zusätzlich zu (5), dass für eine grosse Hauptzugspannung das Verhältnis der Schubspannweite a zur Flanschbreite b möglichst klein gewählt werden sollte.

\section{b) Aufgrund des Flanschfachwerk-Modells}

Die nach diesem Modell im Anschlussbereich auftretende Zugspannung $\sigma_{z}$ beträgt gemäss Gleichung (4)

$$
\begin{aligned}
& \sigma_{z}=\frac{z}{d}=\frac{Q}{4} \cdot \frac{1}{y} \cdot \frac{a}{a-y} \cdot \frac{b-b}{b} \cdot \frac{1}{d}=\frac{Q}{4} \cdot \frac{1}{y} \cdot \frac{a / y}{(a / y)-1} \cdot\left(1-\frac{b o}{b}\right) \cdot \frac{1}{d}, \text { somit } \\
& \sigma_{z}=\frac{Q}{4} \cdot \frac{1}{y \cdot d} \cdot\left(1-\frac{b}{b}\right) \cdot \frac{m}{m-1}
\end{aligned}
$$

Darin bedeutet $m$ die Anzahl der Stegdruckdiagonalen innerhalb a (siehe Bild 9). Aus dieser Formel ist ersichtlich, dass wie bei (5) und (6) bei einer gegebenen Querkraft $Q$ die Spannung $\sigma_{z}$ mit der Abnahme von $y$, $d$ und $b_{0}$ /b zunimmt. Eine weitere Zunahme ergibt sich mit abnehmendem $m$. Dieser Einfluss von $m$ ist in Bild 9 dargestellt.

Ein Vergleich der Ergebnisse für die beiden Modelle zeigt, dass die Beanspruchungen im Anschlussbereich Platte - Steg in ähnlicher Weise durch die verschiedenen Balkenparameter beeinflusst werden. Für die endgültige Wahl der Balkenabmessungen sowie der Längs- und Schubarmierung des Steges mussten selbstverständlich noch zusätzliche, d.h. vor allem Versuchs- und ausführungstechnische Aspekte berücksichtigt werden. 
2.1 Beschreibung

2.1.1 Abmessungen, Armierungen

Die Abmessungen und Armierungen gehen aus den Bildern 10 und 11 hervor.

\section{Abmessungen:}

Die Betonabmessungen waren bei allen fünf Balken gleich (Bild 10). Der Balken war $7.04 \mathrm{~m}$ lang und $62 \mathrm{~cm}$ hoch, der Steg $20 \mathrm{~cm}$ breit. Die Breite und Dicke der Platte betrugen $100 \mathrm{~cm} \mathrm{bzw.} 10 \mathrm{~cm}$.

\section{Stegarmierengng:}

Auch die Biege- und Schubarmierungen waren bei allen Balken dieselbe (Bild 10). Die Biegearmierung bestand aus $6 \emptyset 30 \mathrm{~mm}\left(\mu_{L}=0.77 \%\right)$, die Schubarmierung aus Bügeln von $12 \mathrm{~mm}$ Durchmesser und $10 \mathrm{~cm}$ Abstand $\left(\mu_{\mathrm{B}}=1.13 \%\right)$.

\section{Platțenarmiererung:}

Die nach den entsprechenden Modellen bemessenen und teilweise ergänzten Plattenquerarmierungen (siehe 1.4.1,2.3.3 und 2.3.4) sind in Bild 11 aufgezeichnet. Die Armierung wurde in zwei Lagen eingelegt, d.h. oben und unten in der Platte. Dabei wurde nur ein einziger Stabdurchmesser verwendet, nämlich $\emptyset 6 \mathrm{~mm}$. Die genauen Abstände der Plattenarmierungsstäbe vom näheren Plattenende sind in Tabelle 8 angegeben.

\subsubsection{Herstellung und Lagerung}

Für die Herstellung der Balken Q1 bis Q5 wurde eine Holzschalung verwendet. Die pro Balken notwendige Betonmenge wurde in einem Zwangsmischer in drei Mischchargen hergestellt. Der Beton wurde nach dem Einbringen mit Nadelvibratoren verdichtet. Jeweils eine Woche nach dem Betonieren wurden die Balken ausgeschalt und blieben in der Betonierhalle, wo sie auch für die Prüfung vorbereitet wurden. Diese erfolgte jeweils im Alter von 28 Tagen.

\subsection{Baustoffe}

\subsubsection{Armierungsstahl}

Die Stegarmierung aller Balken war aus naturhartem StahI (Box-Ultra). Die Plattenarmierung bestand hingegen aus einem ursprünglich kaltverformten Stahl (Tor 50, $\emptyset 6 \mathrm{~mm}$ ), der jedoch durch eine spezielle Wärmebehandlung im Werk wieder eine ausgeprägte Fliessgrenze erlangte. Vor den Balkenversuchen durchgeführte konventionelle Zugversuche an fünf Stäben dieser Armierung ergaben im Mittel eine Fliessgrenze van $4.74 \mathrm{t}^{\mathrm{c}} \mathrm{cm}^{2}$ und eine Zugfestigkeit von $5.24 \mathrm{t} / \mathrm{cm}^{2}$. Der Wert von $4.74 \mathrm{t} / \mathrm{cm}^{2}$ wurde für die Dimensionierung der Plattenarmierung verwendet.

Im Anschluss an die Balkenversuche wurden in einer dehnungsgesteuerten Zugmaschine Armierungsstähle der verwendeten Durchmesser ( $\emptyset=6,12$ und $30 \mathrm{~mm}$ ) geprüft. Dabei 
wurde die Dehnungsgeschwindigkeit auf $1.25 \%$ pro Minute festgelegt. Im Fliessbereich und bei der Höchstlast wurde die Dehngeschwindigkeit gleich null gesetzt. Während einer Wartezeit von zwei Minuten fiel der sogenannte dynamische Festigkeitswert ( $\sigma_{f d}$ bzw. $\beta_{z d}$ bei $\dot{\varepsilon} \neq 0$ ) auf den statischen Wert ( $\sigma_{f s}$ bzw. $\beta_{z s}$ bei $\dot{\varepsilon}=0$ ) ab. Wartezeiten länger als zwei Minuten haben keinen Einfluss auf die statischen Festigkeitswerte [4]. Die so erhaltenen Werte sind in Tabelle 4 zusammengestellt. Ein Beispiel der für die Stäbe $\emptyset 6 \mathrm{~mm}$ erhaltenen $\sigma-\varepsilon-0$ iagramme ist in Bild 12 angegeben. In Bild 13 ist das für die Stäbe $\emptyset 6 \mathrm{~mm}$ gemittelte idealisierte $\sigma-\varepsilon-\square i a g r a m m a u f g e z e i c h n e t$.

\subsection{Beton}

Die Zusammensetzung des Frischbetons war für alle fünf Balken dieselbe und ist in Tabelle 1 angegeben. Der Zuschlagstoff, der aus gut gewaschenem und getrocknetem Material bestand, wurde getrennt nach drei Komponenten beigegeben. Die Siebkurve der Mischung entsprach ungefähr der Fuller-Kurve. Als Bindemittel wurde normaler Portlandzement verwendet.

Zur Bestimmung der Betonfestigkeiten wurden von den drei Mischungen jedes Balkens je sechs Prismen $(12 \times 12 \times 36 \mathrm{~cm})$ und zwei Zylinder $(15 \mathrm{~cm} \emptyset$ und $30 \mathrm{~cm}$ Höhe $)$ hergestellt. Im Alter von 28 Tagen (entspricht dem Prüfalter der Balken) wurden pro Versuchsträger auf konventionelle Weise folgende Proben geprüft:

6 Prismen zur Bestimmung der Biegezugfestigkeit $\beta_{b z}$ und der Würfeldruckfestigkeit $\beta^{12}$ (ermittelt an den aus Biegezugversuchen anfallenden Prismenhälften, Druckfläche $12 \times 12 \mathrm{~cm})$. G Prismen zur Bestimmung der Prismendruckfestigkeit $\beta_{p}$. 6 Zylinder zur Bestimmung der Querzugfestigkeit $B_{q z}$.

Die Mittelwerte dieser Festigkeiten sind in Tabelle 2 zusammengestellt. Der Variationskoeffizient in $\%$, der als Mass für die Streuung gilt, ist in Klammern angegeben.

Um das Spannungs-Dehnungs-Diagramm des Betons zu ermitteln, wurde pro Versuchsbalken zusätzlich ein weiteres Prisma im Alter von fünf Wochen geprüft. Die Prüfmethode ist in [5] ausführlich dargestellt und wird hier nur kurz beschrieben. Die Belastung wurde vorerst stufenweise so aufgebracht, dass eine mittlere Betonspannung von $5,25,50$, $75,100,5,100 \mathrm{~kg} / \mathrm{cm}^{2}$ resultierte. Bei den folgenden Laststufen bis zum Bruch wurde jeweils die belastung so verändert, dass eine zusätzliche Stauchung von $0.15 \times 10^{-3}$ während einer Minute aufgebracht und anschliessend die Stauchung während zwei Minuten konstant gehalten wurde. Durch diese Prüfmethode erhält man für Beton ein dynamisches $(\dot{\varepsilon} \neq 0)$ und ein statisches $(\dot{\varepsilon}=0)$ Spannungs-Dehnungs-Diagramm. Ein Beispiel zeigt Bild 14. In Tabelle 3 sind die wichtigsten Ergebnisse dieser Versuche zusammengestellt.

Das gemittelte Spannungs-Dehnungs-Diagramm des verwendeten Betons wurde durch die kubische Parabel

$$
\frac{\sigma_{b}}{\beta_{p}}=\varphi\left(\frac{\varepsilon_{b}}{\varepsilon_{u}}\right)\left[1-\left(2-\frac{3}{\varphi}\right)\left(\frac{\varepsilon_{b}}{\varepsilon_{u}}\right)+\left(1-\frac{2}{\varphi}\right)\left(\frac{\varepsilon_{b}}{\varepsilon_{u}}\right)^{2}\right] \text { angenähert. }
$$

$$
\text { wobei } \varphi=\varepsilon_{u} \cdot \frac{E_{b o}}{\beta_{p}} \text {, gültig für } 1.5<\varphi<3.0 \text {. }
$$

Auch hier wird zwischen einem dynamischen und einem statischen Diagramm unterschie- 
den. Das dynamische Diagramm ist von der Prüfmethode sowie von der Dẹnungsgeschwindigkeit abhängig und wird mit dynamischen Festigkeitswerten $(\dot{\varepsilon} \neq 0)$ gerechnet. In Gleichung $(B)$ wird daher für $\beta_{p}$ die mittlere dynamische Prismenfestigkeit $\beta_{p d}=$ $244 \mathrm{~kg} / \mathrm{cm}^{2}$ (Tabelle 3 ) eingesetzt. Für $\varepsilon_{u}$ wird der Mittelwert $\varepsilon_{u}=1.69 \%$ verwendet. $E_{\text {bo }}$ bzw. $\varphi$ wurde so bestimmt, dass die Betonstauchung bei $100 \mathrm{~kg} / \mathrm{cm}^{2}$ nach $\mathrm{Gl}$. (8) mit dem gemessenen Mittelwert übereinstimmt. Das erhaltene Diagramm a ist in Bild 15 aufgezeichnet.

Das statische Diagramm wird mit einem statischen Festigkeitswert $(\dot{\varepsilon}=0)$ gerechnet. Da für Beton dieser Wert auch von der Dehngeschwindigkeit, d.h. insbesondere von der Wartezeit bei jeder Laststufe abhängig ist, gehört zu jeder Wartezeit ein anderes Diagramm. Für Wartezeiten von 2 Minuten und 30 Minuten wurden die zwei Diagramme b und $c$ gerechnet (Bild 15). Bei b wurde in Gl. (8) ein $\beta_{p s}\left(2^{\prime}\right)=219 \mathrm{~kg} / \mathrm{cm}^{2}$ eingesetzt, bei c ein $\beta_{p s}\left(30^{\prime}\right)=188 \mathrm{~kg} / \mathrm{cm}^{2}$ (Tabelle 3$)$. $E_{\text {bo }}$ und $\varepsilon_{u}$ wurden gleich gross wie beim dynamischen Diagramm angenommen.

\subsection{Bemessung und rechnerische Werte}

\subsubsection{Berechnete Lasten}

Der Bemessung der Balken wurden die mit den vorermittelten (Plattenquerarmierung) bzw. angenommenen Materialkennwerten berechneten theoretischen Last- und Schnittkraftgrössen zugrunde gelegt (vorberechnete Grössen). In Spalte 1 und 3 von Tabelle 7 sind die entsprechenden Längs- und Querbelastungen für den Bruch des Steges ( $P$ Th) und für das Fliessen der Platte $\left(P_{f}^{T h}, \bar{P}_{f}^{T h}\right.$ ) aufgeführt. Die bei diesen Lasten im Steg bzw. im Anschlussbereich Platte - Steg sich ergebenden nominellen Schubspannungen sind in den Spalten 2 und 4 der Tabelle 7 angegeben. Diese nominellen Schubspannungen wurden wie folgt definiert:

- Nominelle Schubspannung im Steg: $\tau_{s}=\frac{Q}{b_{0} \cdot h}=\frac{P^{T h}}{b_{0} \cdot h}$, wobei h = statische Höhe des Trägers $=55 \mathrm{~cm}$.

- Nominelle Längsschubspannung im Anschluss Platte/Steg (siehe Gleichung (5)): $\tau_{x y}=\frac{Q}{2} \frac{1}{y \cdot d}\left(1-\frac{b}{b}\right)=\frac{P}{2 \cdot y \cdot d}\left(1-\frac{b}{b}\right)$, wobei $y=$ Hebelarm der inneren Kräfte im Steg $=$ $50 \mathrm{~cm}$.

- Nominelle vertikale Schubspannung im Anschiuss Platte/Steg:

$$
\begin{aligned}
& \tau_{y z}=\frac{\bar{Q}}{l \cdot h_{P}}=\frac{\bar{P}_{f}^{T h}}{l \cdot h_{P}} \text {, wobei l = Abstand zwischen den Pressenkräften der Querbe- } \\
& \text { lastung }=88 \mathrm{~cm}(\text { siehe Bild } 17), h_{p}=\text { statische Hähe in der Platte }=8.7 \mathrm{~cm} .
\end{aligned}
$$

Die Querbelastung $\bar{P}$ erzeugt im Anschlussbereich keinen Längsschub; sie erzeugt lediglich eine geringe vertikale Schubspannung $\tau_{y z}$, die für $\bar{P}=2.91 \mathrm{t} n u r 3.80 \mathrm{~kg} / \mathrm{cm}^{2}$ beträgt (siehe Tabelle 7).

Zum Zwecke eines direkten Vergleiches mit den Versuchsresultaten enthält Tabelle 7 ferner die mit den effektiv ermittelten Materialkennwerten berechneten Grössen (nachberechnete Grässen). Hierbei wurde in Anlehnung an den Versuchsablauf (Abschnitt 3.2) 
zwischen Anfangs- und Endlasten unterschieden. Für die Längsbelastung. P wurde die Anfangsbruchlast des Steges $P_{u A}^{T h}$ bzw. das zugehörige Biegemoment mittels der dynamischen Fliessgrenze der Biegearmierung $\sigma_{f d}(T a b .4)$ und des dynamischen SpannungsDehnungs-Diagrammes des Betons (Kurve a in Bild 15) gerechnet. Die Endbruchlast $P_{u E}^{T h}$ wurde dagegen aus der statischen Fliessgrenze des Stahles $\sigma_{f s}$ und einem SpannungsDehnungs-Diagramm des Betons entsprechend einer Wartezeit von 30 Minuten bei den verschiedenen Laststufen bestimmt (Kurve $c$ in Bild 15). Diese 30 Minuten entsprechen dem Mittel für die Dauer der Messungen bei den verschiedenen Laststufen der Balkenversuche.

Diese Berechnung ergibt bei den Balken $Q 1$ und $Q 2$ eine Anfangsbruchlast ( $P$ Th ) von 61 t und eine Endbruchlast $\left(P_{U E}^{T h}\right)$ von 57 t. Bei den Balken Q3, Q4 und $Q 5$ sind die entsprechenden Bruchlasten infolge der in den Druckplatten für die Querbelastung angeordneten Zugstangen-Aussparungen etwas tiefer, nämlich $P_{u A}^{T h}=60 t b z w$. $P_{u E}^{T h}=55 t$ (Schwächung der Druckzone).

Analog wie für die Stegbruchlasten wurden für die Balken Q1 und Q2 die Lasten für den Fliessbeginn der Plattenarmierung $P_{f A}^{T h}$ und $P_{f E}^{T h}$ nachberechnet. Sie betragen $44.2 t$ bzw. 42.7 t. Diese Werte gelten auch für die Balken Q3, Q4 und Q5, sofern gleichzeitig eine Querbelastung $\bar{P}_{f A}^{T h}$ bzw. $\bar{P}_{f E}^{T h}$ wirkt.

Die Querbiegebelastung $\bar{P}_{f}^{T h}$, die bei den Balken $Q 3$, Q4 und $Q 5$ zum Fliessen der Plattenarmierung im Bereich reiner Biegung führt, wurde ebenfalis auf diese Art nachberechnet. $\vec{P}_{f A}^{T h}$ beträgt $3.0 \mathrm{t} /$ Kolben, $\bar{P}_{f E}^{T h} 2.92 \mathrm{t} /$ Kolben (Kap. 3). Die nachgerechnete Fliesslast der Plattenarmierung $\bar{P}_{f A}^{T h}$ liegt nur ca. $3 \%$ über derjenigen, welche mit angenommenen Materialkennwerten ermittelt wurde $\left(\bar{P}_{f}^{T h}=2.91\right.$ t/Kolben), und die beim Versuch tatsächlich aufgebracht worden war.

\subsubsection{Bemessung der Stegarmierung}

Da die Hauptzugspannung bzw. die Zugspannung im Anschlussbereich Platte/Steg mit der Querkraft zunimmt (siehe Abschnitt 1.4.2), wurden die Balken auf eine möglichst grosse Querkraft bemessen. Als "Bemessungslast" der Balken wurde daher jene Last angenommen, unter der die nominelle Schubspannung im Steg ( $\left.\tau^{*}\right)$ gleich gross wird wie die obere Schubspannungsgrenze $\left(5 \tau_{1}^{*}\right)$ nach Richtlinie 17 der Norm SIA 162 [6]. Bei den gewählten Querschnittsabmessungen (Bild 10) sowie mit einer angenommenen Würfeldruckfestigkeit von $\beta_{w}=300 \mathrm{~kg} / \mathrm{cm}^{2}$, wird die obere Schubspannungsgrenze bei einer Last $P=55 \mathrm{t}$ erreicht (Eigengewicht vernachlässigt). Da bei den Balken mit Querbiegung (Q3 $\div$ Q5) die Querbelastung $\bar{P}$ als "Eigenspannungszustand" des Querschnittes aufgebracht wurde (s. Abschnitt 3.1) erzeugt sie im Steg keine Querkraft. Daher wurde die Schubarmierung des Steges bei allen Balken nach der oben erwähnten Richtlinie für $P=55 t$ bemessen. Die Biegearmierung liess sich für die gleiche Belastung nach den üblichen Regeln der plastischen Bemessung bestimmen (siehe z.B. [3]). Dabei wurde für Stahl eine Fliessspannung von $5.2 \mathrm{t} / \mathrm{cm}^{2}$ sowie für Beton eine Würfeldruckfestigkeit von

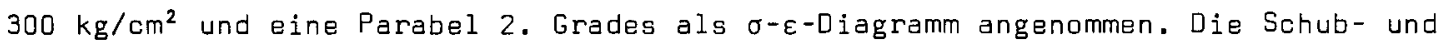
Biegearmierungen des Steges sind aus Bild 10 ersichtlich. Der Armierungsgehalt für die Längsarmierung $\mu_{L}$ beträgt $0.77 \%$, derjenige der Schubarmierung $\mu_{B} 1.13 \%$. 


\subsubsection{Bemessung der Plattenarmierung}

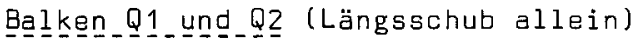

Beim Balken Q1 wurde die Plattenarmierung nach dem Klassischen "HauptzugspannungsModell" bemessen. Die Armierung wurde so dimensioniert, dass sie wie beim Balken Q2 bei einer Last von $P=42.8 \mathrm{t}$ (siehe unten) zum Fliessen kommen sollte.

Der Balken Q2 wurde wie folgt bemessen: Nach Fachwerkmodell (s. Abschnitt 1.3.1) wirkt im Anschlussbereich pro Längeneinheit eine Zugkraft

$$
z=\frac{Q}{4 y} \cdot \frac{a}{a-y} \cdot \frac{b-b}{b}
$$

Wertet man diesen Ausdruck für die vorliegenden Versuchsträger aus, so wird die Zugkraft $Z=\frac{16}{15} \cdot \frac{Q}{4 y}$. Für die Dimensionierung der Plattenarmierung wurde indessen von $Z=\frac{Q}{4 y}$ ausgegangen. Weil Fliessen der Armierung zuerst in der Platte und nicht im Steg erfolgen sollte, wurde die Plattenarmierung so bemessen, dass sie bei einer Last von etwa $75 \%$ der Biege- bzw. der rechnerischen Stegbruchlast fliessen sollte. Es wurde eine zweilagige Plattenarmierung von $2 \times 8 \emptyset 6 \mathrm{~mm}$ pro Meter Balkenlänge gewählt, d.h. $2 \square 6 \mathrm{~mm}$ im Abstand $t=12.5 \mathrm{~cm}$. Diese Armierung fliesst bei einer Belastung $P$ bzw. einer Querkraft $Q=4 y \times$ Fliesskraft der Armierung pro Meter Balkenlänge. Mit $y \simeq 50 \mathrm{~cm}$ und $\sigma_{f}=4.74 \mathrm{t} / \mathrm{cm}^{2}$ errechnet sich diese Belastung $z u P=42.8 \mathrm{t}$.

Die Plattenarmierungen der Balken Q1 und Q2 sind in Bild 11a dargestellt.

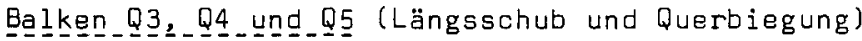

In denjenigen Plattenteilen längs dieser drei Träger, wo infolge der Längsbelastung $P$ gemäss den Modellen (siehe 1.3.1) im Anschlussbereich keine Längsschubbeanspruchung entsteht, wurde oben in der Platte einzig eine Armierung von $10 \emptyset 6 \mathrm{~mm} / \mathrm{m}$ zur Aufnahme des Querbiegemomentes eingelegt. Das Fliessmoment (bei $\varepsilon_{e}$ max $=\varepsilon_{f}$ ) dieses Plattenquerschnittes betrug $1.09 \mathrm{mt} / \mathrm{m}^{\prime}$. Der Armierungsgehalt $\mu=F_{e} /$ bh der Platte im Bereich reiner Biegung ergibt sich zu $0.32 \%$.

In den übrigen Plattenteilen, wo zur Querbiegung noch Längsschub aus der Längsbelastung wirkt, wurden die Beanspruchungen im Anschlussbereich jeweils nach einem der im Abschnitt 1.3.2 dargestellten Modelle berechnet. Die Plattenarmierung in diesen Bereichen wurde bei allen drei Balken so bemessen, dass sie - immer gemäss den betreffenden Modellen - unter einer Längsbelastung $P=42.8 \mathrm{t}$ und einer Querbelastung $\bar{P}=$ $2.91 \mathrm{t} /$ Kolben (entspricht einer Querbelastung von $3.31 \mathrm{t} / \mathrm{m}^{\prime}$ ) zum Fliessen kommen sollte.

Die Plattenarmierungen der Balken Q3, Q4 und Q5 sind in Bild 11b dargestellt.

\subsubsection{Konstruktive Ergänzungen der Modellarmierungen}

Bei den vier Balken Q1, Q3, Q4 und Q5 wurde die Armierung sowahl bezüglich Stärke als auch bezüglich Lage genau den entsprechenden Bemessungsmodellen angepasst. In den Bereichen, wo nach den Modellen keine Armierung notwendig gewesen wäre, wurde oben und unten in der Platte eine konstruktive Minimalarmierung ( $(6 \mathrm{~mm}, t=27.5 \mathrm{~cm}$ ) angeordnet, was einem $\mu=0.12 \%$ entspricht. 
armierung $(2 \emptyset 6 \mathrm{~mm}$ je $12.5 \mathrm{~cm}$ ) über die ganze Länge der Platte durchgezogen.

Ein Vergleich zwischen den nach den Modellen erforderlichen Armierungen und denjenigen, die effektiv eingelegt wurde, ergibt sich aus Bild 16. 


\section{VERSUCHSDURCHFUEHRUNG}

\subsection{Versuchsanlage}

Die Balkenversuche wurden auf dem Aufspannboden in der Prüfhalle der Eidgenössischen Materialprüfungs- und Versuchsanstalt (EMPA) in Dübendorf (ZH) durchgeführt. Die Balken wurden auf zwei blockierbare Rollenlager gesetzt, die abwechslungsweise gelöst bzw. blockiert wurden, um im Laufe des Versuchs eine symmetrische und zwängungsfreie Verschiebung der beiden Balkenauflager zu gewährleisten. Die Belastung $P$ zur Erzeugung des Längsschubes - jeweils "Längsbelastung" genannt - erfalgt durch zwei 100 tDruckpressen in den Drittelspunkten der Spannweite (Bild 17). Damit die Druckkolben auch bei zunehmender Balkenkrümmung zwängungsfrei arbeiten konnten, wurde zwischen dem Versuchsbalken und den Pressen ein Lastverteilträger auf zwei Rollenlagern eingebaut. Um das Abrollen dieses Verteilträgers zu verhindern, wurde er mittels einer Rohrstange an einem festen Punkt ausserhalb des Versuchsbalkens festgehalten. Eine Gesamtansicht der Versuchsanlage für die Balken Q1 und Q2 zeigt Bild 18.

Um bei den Balken Q3, Q4 und Q5 in der Platte ein Querbiegemoment zu erzeugen, wurden pro Balken symmetrisch zur Balkenaxe und im Abstand von $88 \mathrm{~cm} 16$ Zugkolben von je $15 \mathrm{t}$ Höchstlast eingebaut (Bilder 19 und 17). Diese wurden an eine zweite unabhängige Pumpenanlage angeschlossen. Jeder Kolben zieht die Platte mit der Kraft $\bar{P}$ - jeweils "Querbelastung" genannt - nach unten, wobei die Reaktion dieser Kraft von unten auf den Steg des Plattenbalkens wirkt. Je zwei einander gegenüberliegende Kräfte $\bar{P}$, die am Plattenrand angreifen, bilden mit der Reaktion $2 \bar{P}$ gewissermassen einen Eigenspannungszustand und verursachen somit in der Längsrichtung des Balkens keine Schnittkräfte, bzw. keine zusätzlichen Längsschubspannungen $\tau_{y x}$ im Anschlussbereich. Abgesehen von versuchstechnischen Vorteilen wird damit ein direkter Vergleich zwischen den Balken mit "Längsschub und Querbiegung" und jenen mit "Längsschub allein" möglich.

Um zu erreichen, dass im Anschlussbereich der Platte längs des Balkens möglichst ein konstantes Querbiegemoment entsteht, wurde die Anzahl der Einleitungspunkte für die Zugkräfte $\bar{P}$ durch kleine Lastverteilträger HEB 100 verdoppelt.

\subsection{Laststufen und Versuchsablauf}

Vorerst sollen für die Längsbelastung $P$ wie auch für die Querbelastung $\bar{P}$ die gewählten wichtigsten Laststufen angegeben werden. Diese sind:

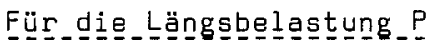

$P_{I}=P_{I I I} / 1.8 \simeq 23.6 t$
$P_{I I}=P_{I I I} / 1.3 \simeq 33.2 t$
$P_{I I I}=P_{f}^{T h}$

wobei $P_{f}^{T h}=$ Theoretische Ladt für Fliessen der Plattenarmierung im Falle von Längsschub allein (s. Tabelle 7, Spalte 3 und Abschnitt 2.3.3*)).

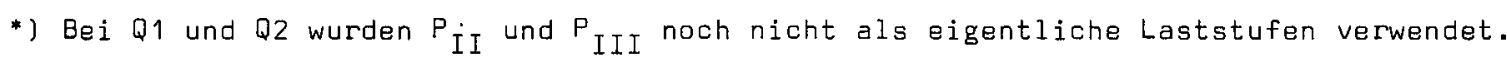
Die Versuchsresultate für $P_{\text {II }}$ und $P_{\text {III }}$ mussten daher aus benachbarten Laststufen interpoliert werden. 


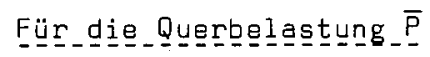

$\bar{P}_{I}=\bar{P}_{I I I} / 1.8 \simeq 1.64 \mathrm{t} /$ Kolben

$\bar{P}_{I I}=\bar{P}_{I I I} / 1.3 \simeq 2.28 \mathrm{t} /$ Kolben

$\bar{P}_{I I I}=\bar{P}_{f}^{T h} \simeq 2.91 \mathrm{t} /$ Kolben,

wobei $\bar{P}_{f}^{T h}=$ Theoretische Last für Fliessen der Plattenarmierung im Bereich reiner Biegung (s. Tabelie 7, Spalte 3 und Abschnitt 2.3.3).

Die Längsbelastung $P$ wurde stufenweise aufgebracht. Am Anfang des Versuches wurden die Laststufen durch gegebene, von vorneherein bestimmte Lasten festgelegt (lastgestevert). Später und bis zum Bruch der Balken wurde die Belastung jeweils so verändert, dass die Durchbiegung in Balkenmitte um einen bestimmten Betrag zunahm (deformationsgesteuert). Dieser Betrag variierte zwischen drei und zehn Millimetern. Die Umstellung von last- auf verformungsgesteuerte Versuchsdurchführung erfolgte bei den Balken $Q 1$ und $Q 2$ bei $P=39 t$, bei $Q 3, Q 4$ und $Q 5$ bei $P=P_{\text {III }}=42.8 \mathrm{t}$.

Die Querbelastung $\bar{P}$ wurde während des ganzen Versuchsablaufs lediglich auf die drei oben erwähnten Lasten $\bar{P}_{I}, \bar{P}_{I I}$ und $\bar{P}_{\text {III }}$ gebracht (lastgesteuert), ohne Einschaltung von Zwischenstufen.

Bei $P=P_{I}=23.6 \mathrm{t}$ (Q1 und $\left.Q 2\right)$, bzw. $P_{I}=23.6 t$ und $\bar{P}_{I}=1.64 t$ (Q3-Q5) wurden zehnmal Belastungs- und Entlastungszyklen zwischen diesen Lasten und den Grundlasten eingeschaltet um das Verhalten im Gebrauchszustand bei wiederholter Belastung zu erfassen.

Als Beispiele für die Belastungsprogramme sind diejenigen für die Balken Q1 (Längsschub allein) und Balken Q4 (Längsschub und Querbiegung) in den Bildern 21 und 22 angegeben. Die Anfangs- und Endlasten $P_{A}$ und $P_{E}$, bzw. $\bar{P}_{A}$ und $\bar{P}_{E}$ sind für die Balken $Q 1$ bis $Q 5$ in den Tabellen 5 und 6 aufgéführt.

Bei jeder Laststufe kam für die Längsbelastung $P$ wie auch für die Querbelastung $\bar{P}$ folgendes Vorgehen zur Anwendung (siehe Bild 20):

Phase 1: Verändern der Last innerhalb van ca. 2 Minuten bis zum Erreichen der vorgesehenen Last- bzw. Verformungsgrösse. Ablesen der Anfangslast $P_{A}$ resp. $\bar{P}_{A}$.

Phase 2: Konstanthalten der Anfangslast $P_{A}$ resp. $\bar{P}_{A}$ während 2 Minuten.

Phase 3: Konstanthalten einer Deformationsgrässe (für P Durchbiegung in Balkenmitte, für $\bar{P}$ relative Durchbiegung der Platte zum Steg) für die Dauer der Messungen (30 - 60 Minuten); infolge Relaxation entsprechender Abfall der Last. Nach Beendigung der Messungen Ablesen der Endlast $P_{E}$ bzw. $\bar{P}_{E}$ dieser Laststufe.

Die Prüfung der Balken erfolgte im Alter von 28 Tagen und dauerte jeweils zwei bis drei Tage. 


\subsubsection{Lasten}

Die in den Balken wirksamen Schnittkräfte wurden aus den gemessenen Lasten $P$ und $\bar{P}$ ermittelt. Diese konnten jeweils an den Pendelmanometern der zwei voneinander unabhängigen Pumpenanlagen abgelesen werden. Die aufgebrachte Querbelastung $\bar{P}$ wurde zusätzlich noch durch elektrische Dehnmessstreifen an den Verbindungsstangen zwischen der Platte und den Zugkolben kontrolliert.

\subsubsection{Verformungen}

Es wurden folgende Verformungsmessungen durchgeführt:

Stegdurchbiegung: Die Durchbiegung des Steges wurde jeweils in der symmetrieebene des Balkens an fünf Stellen mittels festverdrahteter induktiver Weggeber gemessen (Ablesegenauigkeit $0.005 \mathrm{~mm}$ ).

Plattendurchhbiegung: Entlang des Balkens wurde die Plattendurchbiegung im seitlichen Abstand von $5 \mathrm{~cm}$ vom Steg, bzw. Plattenrand, ermittelt (Bild 24). Hierfür wurde ein spezielles elektronisches Deformeter mit grosser veränderlicher Basis verwendet (Ablesegenauigkeit $0.01 \mathrm{~mm}$ ).

Qehnung der_Längsarmierung: Bei allen fünf Balken wurde die Dehnung eines Eckstabes über die Hälfte der Balkenlänge gemessen (Bild 23).

Qehnung_der_eügelarmierung: Die Bügeldehnung wurde ebenfalls entlang einer Balkenhälfte auf einer Stegseite gemessen (Bild 23).

Betonstauchung: An der oberen und unteren Seite der Platte wurde jeweils die Betonstauchung gemessen (Bilder 25 - 29). Die inneren Messreihen waren $3 \mathrm{~cm}$ vom Steg entfernt, die äusseren $3 \mathrm{~cm}$ vom Plattenrand. Die Dehnungs-bzw. Stauchungsmessungen an Längs- und Bügelarmierungen bzw. am Beton wurden mit einem elektronischen Deformeter von $20 \mathrm{~cm}$ Basislänge und $0.001 \mathrm{~mm}$ Ablesegenauigkeit vorgenommen.

Dehnung der_Plattenarmierung: Die Dehnung der oberen und unteren Plattenarmierung wurde in verschiedenen Querschnitten über die Balkenlänge gemessen (Bild 30). Die Lage der Messstellen im Messquerschnitt bei den fünf untersuchten Balken Q1-Q5 geht aus den Bildern 31, 32, 34, 35 und 36 hervor. Während die Messung der Dehnungen der oberen Plattenarmierung im interessierenden Bereich Platte - Steg sehr gut möglich war (z.B. Bild 31b), konnte an der unteren Armierung erst im Abstand von $10 \mathrm{~cm}$ (Mitte der Messstrecke) rechts bzw. links des Steges die Dehnung erfasst werden. Die Dehnung der Plattenarmierung wurde mit einem Deformeter von $10 \mathrm{~cm}$ Basislänge und $0.001 \mathrm{~mm}$ Ablesegenauigkeit gemessen.

Bei sämtlichen Messungen mittels Deformetern wurden diese auf Messbolzen aufgesetzt, die mit einem Schnellklebestoff auf der freiliegenden Stahl-bzw. Betonoberfläche aufgeklebt waren. Mit diesem Verfahren konnten die Messungen bis zum Bruch der Balken einwandfrei vorgenommen werden. 


\subsubsection{Risse}

Die in der Platte und im Steg auftretenden Risse wurden mit schwarzem Filzstift nachgezeichnet. Die Breiten der Risse in der Platte wurden jeweils mit einem Rissemassstab ausgemessen. Die ermittelten Werte wurden - wenn > 0.05 mm - auf klebern notiert und neben den Messpunkten fixiert. Das Rissebild und damit auch die Rissbreiten wurden bei jeder Laststufe photographisch festgehalten (z.B. Bilder 43 und 51 ). 
4. VERSUCHSRESULTATE

In diesem Kapitel werden die Resultate der fünf untersuchten Balken wiedergegeben. Die Druckplatten der Balken $Q 1$ und Q2 ("Längsschub allein") wurden nach dem Hauptzugspannungs- bzw. Fachwerk-Modell konzipiert. Die Platten der übrigen Balken, Q3, Q4 und Q5 ("Längsschub mit Querbiegung") wurden nach folgenden Modellen bemessen: Q3 nach dem Modell "Superposition der Armierungen aus Hauptzugspannungs-Modell und Querbiegung", Q4 nach dem Modell "Superposition der Armierungen aus Flanschfachwerk-Modell und Querbiegung" und Q5 nach dem Modell "Armierung für Querbiegung mit Querzugkraft aus Flanschfachwerk-Modell". Zur Erzeugung des Längsschubes wurden die Balken durch zwei Einzellasten in den Drittelspunkten der Spannweite belastet (Bild 18). Bei den Balken Q3 - Q5 wurde zudem eine Querbiegebelastung der Platte aufgebracht (Bild 19).

4.1 Allgemeines Tragverhalten

Beim Balken $Q 1$ trat der erste Riss von der Balkenmitte hergesehen etwas ausserhalb der Einzellast bei einer Längsbelastung von $P=35 \mathrm{t}$ auf (Bild 42). Diese Anrisslast, welche im Auflagerquerschnitt eine rechnerische Hauptzugspannung $\sigma_{\mathrm{hz}}$ von $26 \mathrm{~kg} / \mathrm{cm}^{2}$ im Anschlussbereich Platte - Steg erzeugt, liegt höher als die im gleichen Querschnitt mit $\sigma_{h z}=20 \mathrm{~kg} / \mathrm{cm}^{2}$ gerechnete "theoretische" Anrisslast von $27 \mathrm{t}$ (Tabelle 7). Das nachfolgende Verhalten dieses Balkens war sehr ungünstig; die maximale Rissbreite betrug bei der Höchstlast $3 \mathrm{~mm}$. Der Bruch erfolgte schliesslich wegen der aufgrund des klassischen Modells falsch plazierten und erheblich geflossenen Plattenarmierung durch Abscheren der Platte vom Steg (Bild 45). Die Höchstlast des Balkens liegt unter der rechnerischen Biegebruchlast (Tabelle 7).

Beim Balken Q2 trat der erste Riss praktisch an der gleichen Stelle auf wie beim Balken $Q 1$ (Bild 46). Die für diese Stelle nach dem Fachwerk-Modell ( $\left.P=Q=4 \cdot y \cdot d \cdot \sigma_{z}\right)$ gerechnete und die experimentelle Anrisslast stimmen hier gut überein ( $P_{R}$ gerechnet $=40 \mathrm{t}, P_{R}$ gemessen $=37 \mathrm{t}$, siehe Tabelle 7 ). Für die Berechnung der Anrisslast wurde analog wie bei $Q 1$ für die Zugfestigkeit des Betons der Wert $20 \mathrm{~kg} / \mathrm{cm}^{2}$ eingesetzt. In der Folge verhielt sich der Balken wesentlich besser als Q1. Bei der Höchstlast betrug die maximale Rissbreite nur $0.3 \mathrm{~mm}$. Die Plattenarmierung gemäss Fachwerk-Modell verhinderte ein Abscheren der Platte vom Steg solange, bis in Balkenmitte ein Biegebruch auftrat (Bild 49). Die erreichte Höchstlast entspricht nahezu der rechnerischen Biegebruchlast.

Beim Balken Q3 entstanden erwartungsgemäss infolge Querbiegung schon relativ früh die ersten Risse. Die Plattenarmierung floss zuerst in der Nähe der Einzellasten. wo gemäss Modell nur wenig Armierung eingelegt war. Dies trat bei einer Belastung $P=33.2 \mathrm{t}, \bar{P}=2.91 \mathrm{t}$ ein; demgegenüber betragen die theoretischen Fliesslasten $P=44.2 t, \bar{P}=3.0 \mathrm{t}$. Dank Umlagerung der Kräfte in der Plattenarmierung konnte je doch die Längsbelastung noch wesentlich gesteigert werden. Das Risseverhalten dieses Balkens war indessen ziemlich ungünstig, bei der Höchstlast betrug die maximale Rissbreite $1.1 \mathrm{~mm}$.

Die Balken Q4 und Q5 zeigten gegenüber Q3 ein besseres Risse- und Tragverhalten. Die Fliesslast $P_{f A}^{E X}$ der Plattenarmierung lag wesentlich höher: $51.2 \mathrm{t}$ bei Balken $Q 4$ und 47.5 t bei Balken Q5 (s. Tabelle 7). Unter der Höchstlast betrug die maximale Rissbreite $0.3 \mathrm{~mm}$ bei Balken $Q 4$ und $0.4 \mathrm{~mm}$ bei Balken $Q 5$. 


\subsection{Traglasten}

Die maximalen Anfangswerte der Längsbelastung $P_{U A}^{E x}=P_{U}$, und die zugehörigen Endwerte $P$ EE (vergleiche Bilder 21 und 22 ) sind für die fünf geprüften Balken $Q 1$ bis Q5 in Tabelle, 8 zusammengestellt.

Der Vergleich zwischen der Höchstlast $P_{u}$ und der entsprechenden nachgerechneten Anfangslast $P_{u A}^{T h}$ bei den verschiedenen Trägern zeigt, dass $P_{u}$ stets tiefer liegt als $\mathrm{P}_{u A}^{T h}$. Bei den Balken $\mathrm{Q} 2$ bis $Q 5$, welche Biegebrüche aufwiesen, ist diese Unterschreitung auf folgende drei Faktoren zurückzuführen:

1. Bei den in Tabelle 7 angegebenen Stegmaximallasten ist das Eigengewicht des Balkens und der Versuchseinrichtungen nicht berücksichtigt. Dieses entspricht bezüglich des maximalen Momentes einer Längsbelastung von etwa $P=1.5$ t. Bei dem Vergleich zwischen Versuch und Rechnung sollten daher die gemessenen Werte um $1.5 \mathrm{t}$ erhöht werden.

2. Die Biegearmierung im Querschnitt dürfte etwas häher gelegen haben als theoretisch angenommen. Somit wird der tatsächliche Hebelarm der inneren Kräfte etwas kleiner.

3. Die Dehngeschwindigkeit des Stahles war während der Lasterhähung bei den Balken ( 0.1 \%o/Min.) erheblich kleiner als jene bei den Zugversuchen (1.25\%/Min.), an denen die für die Rechnung benützte Fliessspannung $\sigma_{f d}$ ermittelt wurde. Da $\sigma_{f d}$ mit kleinerer Dehngeschwindigkeit abnimmt, ist die in der Berechnung von $P_{U A}^{T h}$ eingesetzte Fliessspannung grösser als die effektive bei den Balken.

4. Die theoretischen Werte $P_{U A}^{T h}$ (auch $P_{U E}^{T h}$ ) wurden unter der Annahme "Ebenbleiben der Querschnitte" gerechnet, welche beim Plattenbalken bekanntlich nicht zutrifft (ungleichmässige Verteilung der Beanspruchungen der Druckplatte).

Beim Balken Q1, bei dem keine Biegebruch auftrat, liegt $P_{U}\left(=P_{U A}^{E x}\right)$ wesentlich tiefer als $P_{U A}^{T h}$. Die Ursache hiefür ist neben den oben erwähnten Faktoren - die Bruchart dieses Trägers: er versagte infolge eines Schubbruchs in der Platte (Abscheren der Platte vom Steg).

Für die Querbelastung $\bar{P}$ gibt es bei den Belken mit Querbiegung (Q3 bis Q5) keine entsprechenden maximalen Lasten, da $\overline{\mathrm{P}}$ nicht über"die theoretische Fliesslast der Platte $\left(\bar{P}_{f}^{T h}=\bar{P}_{\text {III }}=2.91\right.$ t/Kolben $)$ hinaus gesteigert wurde.

\subsection{Plattenfliesslasten}

In Tabelle 7 sind die Lasten $P_{f A}^{E x}$ bzw. Lastkombinationen $P_{f A}^{E x}$, $\bar{P}_{f A}^{E x}$ jener Laststufen aufgeführt, bei denen in den Balkenbereichen "Längsschub allein" (Q1, Q2) bzw. "Längsschub mit Querbiegung" (Q3, Q4, Q5) die Plattenarmierung erstmals zum Fliessen kam.

Beim Balken Q1 floss die Plattenarmierung bei der theoretischen Fliesslast: $P$ fA $=$ $42.8 \mathrm{t}, \mathrm{P}_{\mathrm{fA}}^{\mathrm{Th}}=44.2 \mathrm{t}$. $\mathrm{P}_{\mathrm{fA}}^{\mathrm{Ex}}$ konnte nur deshalb so hoch ausfallen, weil die Platte bis $P=35 t$ ungerissen war, und die Armierung erst mit dem Fortschreiten der Rissebildung einen grösseren Teil der Querzugkräfte aufnahm. Der Balken Q3 zeigte demgegen- 
über ein anderes Verhalten: bei einer Querbelastung $\bar{P}=2.91 \mathrm{t} /$ Kolben war die experimentelle Fliesslast bedeutend tiefer als die theoretische $\left(P_{f A}^{E x}=33.2 t, P_{f A}^{T h}=44.2 t\right)$. Die Platte war infolge der Querbiegung fast von Anfang an gerissen, so dass die entstehenden Querzugkräfte weitgehend von der Armierung übertragen werden mussten.

Bei den Balken Q2, Q4 und Q5 waren die experimentellen Fliesslasten stets höher als die theoretischen (vgl. Tabelle 7). Bei Q2 trat das Fliessen der Plattenarmierung sogar erst bei der Höchstlast des Balkens ein. Dies dürfte auch damit zusammenhängen, dass bei diesem Balken die konstruktive Ergänzungsarmierung sehr stark war, d.h. die Modellarmierung über den ganzen Balken "durchgezogen".

\section{4 Verformungen}

\subsubsection{Allgemeines}

Die Verformungen der verschiedenen Laststufen werden in diesem Bericht der jeweiligen Anfangslast zugeordnet. Charakteristische Lasten bzw. Lastkombinationen für die Darstellung der Versuchsresultate sind:

\section{Balken Q1 und Q2}

Gebrauchslast der Platte: $P_{I}=\frac{P I I}{1.8} \simeq 23.6 \mathrm{t}$

Nächste Laststufe nach Anreissen der Platte: $P_{\Gamma}=$ Versuchsresultat

Theoretische Fliesslast der Plattenarmierung: $P_{f}^{T h}=P_{\text {III }} \simeq 42.8 \mathrm{t}$

Höchstlast des Versuches: $P_{U A}^{E X}=P_{U}=$ Versuchsresultat

Balken Q3, Q4, Q5

Konstantes Querbiegemoment im Anschlussbereich: $\bar{P}_{I}=\frac{\bar{P}_{I I I}}{1.8} \simeq 1.64 \mathrm{t} ; P=1.0 \mathrm{t}$ Gebrauchslasten der Platte: $\bar{P}_{I}=\frac{\bar{P}_{I I I}}{1.8} \simeq 1.64 t ; P_{I}=\frac{P_{I I I}}{1.8} \simeq 23.6 \mathrm{t}$

Laststufe zwischen Gebrauchs- und Fliesslasten der Platte:

$$
\bar{P}_{I I}=\frac{\bar{P}_{I I I}}{1.3} \simeq 2.28 \mathrm{t} ; P_{I I}=\frac{P_{I I I}}{1.3} \simeq 33.2 \mathrm{t}
$$

Theoretische Fliesslasten der Plattenarmierung:

$$
\bar{P}_{f}^{T h}=\bar{P}_{I I I} \simeq 2.91 \mathrm{t} ; P_{f}^{T h}=P_{I I I} \simeq 42.8 t
$$

Höchstlasten des Versuches: $\bar{P}_{f}^{T h}=\bar{P}_{I I I} \simeq 2.91 \mathrm{t} ; P_{U A}^{E X}=P_{U}=$ Versuchsresultat

Bei allen diesen Lasten bzw. Lastkombinationen, mit Ausnahme von $P_{\text {II }}$ bei den Balken $Q 1$ und Q2, waren Laststufen vorhanden, d.h. die darzustellenden Verformungen sind direkt gemessen warden.

Bei den Balken Q1 und Q2 wurde die Dehnung der Plattenarmierung bei P III $\simeq 42.8 t$ durch lineare Interpolation zwischen den benachbarten Laststufen erhalten.

In den Bildern, in denen Stahldehnungen dargestellt sind (z.B. Bild 23, 31a-d), 
wurde jeweils als Vergleichsgrässe die Dehnung des Stahles beim Fliessbeginn aufgetragen. Da die gemessenen Stahldehnungen mittlere Dehnungen über bestimmte Messstrecken sind, müssen sie mit einer ebenfalls "mittleren" Dehnung beim Fliessbeginn verglichen werden. Diese wurde durch Multiplikation der tatsächlichen Fliessdehnung $\varepsilon_{f}=\sigma_{f} / E_{e}$ mit dem Wert des Verbundkoeffizienten $\kappa$ erhalten. $\kappa$ wurde mit 0.9 angenommen (siehe Anhang).

\subsubsection{Dehnungen der Stegarmierung}

\section{Längsarmierung}

Als Beispiel für die gemessenen Dehnungen eines Eckstabes der aus $6 \square 30$ mm bestehenden Biegearmierung sind diejenigen von Balken $Q 2$ in Bild 23 angegeben.

\section{Bügelarmierung}

Von den an einem Bügel an zwei Messstrecken gemessenen Dehnungen wurde jeweils der grössere Wert als massgebend für die Bügelbeanspruchung betrachtet. Als Beispiel sind wiederum für den Balken Q2 die Bügeldehnungen in Bild 23 dargestellt. Man sieht daraus, dass die maximale Bügeldehnung bei der Höchstlast $P_{u}$ unmittelbar bei der Fliessdehnung des Stahles liegt.

\subsubsection{Durchbiegungen des Steges und der Platte}

Bei allen fünf Balken stimmten die neben dem Steg gemessenen Durchbiegungen $\delta_{s}$ (Bild 24) sehr gut mit den in Stegmitte gemessenen überein, so dass nur die ersteren dargestellt wurden. Als Beispiel dient der Balken $Q 4$. Die Stegdurchbiegung $\delta_{s}$ längs des Balkens ist in Bild 24 angegeben.

Die relative Plattendurchbiegung $\delta_{r}$ - als Differenz der absoluten Durchbiegung des Plattenrandes und des Steges ermittelt - ist für die Balken Q1 und Q4 bei verschiedenen Belastungen ebenfalls in Bild 24 aufgetragen. Im Maximum beträgt $\delta_{r} c a .0 .8 \mathrm{~mm}$ beim Balken Q1 (Längsschub allein) und ca. $2.0 \mathrm{~mm}$ beim Balken Q4 (Längsschub und Querbiegung). Beim Balken $Q 1$ wird $\delta_{r}$ durch die Umlenkkräfte der Druckkraft in der durch die Balkendurchbiegung verformten Platte erzeugt. Der Vollständigkeit halber sei noch erwähnt, dass die in Bild 24 dargestellten Durchbiegungen stets Mittelwerte aus den Messungen an beiden Plattenhälften sind.

\subsubsection{Betonstauchungen in der Platte}

Der Verlauf der gemessenen Stauchung entlang der äusseren und inneren Messreihen der oberen und unteren Seite der platte ist für die fünf Balken in den Bildern 25 - 29 dargestellt. Die aufgetragenen Werte sind jeweils das Mittel aus je zwei bezüglich Balkenachse symmetrischen Messungen. Es sind die Stauchungen bei der theoretischen Fliesslast der Platte und bei der Höchstlast des Trägers aufgetragen. Bei den Balken Q1 und Q2 war genau bei $P_{\text {III }}\left(P_{\text {III }}=42.8 \mathrm{t}\right.$ ) keine Laststufe vorhanden, weshalb jeweils die Stauchungen bei der nächsten Laststufe dargestellt wurden $(P=44 \mathrm{t}$ bzw. $P=45 t$, Bilder 25 und 26). Beim Balken Q2 wurden ausserdem infolge Ausfalls einiger Messstellen bei $P_{u}\left(P_{u}=56.1 \mathrm{t}\right)$ die Stauchungen der vorangegangenen Laststufe $(P=56.0 t)$ dargestellt.

Interessant sind die Unterschiede zwischen den Stauchungen beim Steg (innen) und am Plattenrand (aussen). Man erkennt, dass bei allen Balken von den Auflagern her gegen die Lasten $P$ zu die Stauchung beim Steg gegenüber jener am Plattenrand immer stärker überwiegt. Hingegen sind in Balkenmitte bei den Balken ohne Querbiegung (Q1 und Q2) 
die Verhältnisse gerade umgekehrt, d.h. beim Steg ist die Stauchung kleiner als am Plattenrand. Dies ist eine Folge der Plattenverformung. Die horizontale Biegelinie einer Plattenhälfte nimmt etwa die Form des Stahldehnungsverlaufs an (z.B. Bild 31a). Infolge dieser Biegewirkung wird in Balkenmitte die Stauchung beim Steg verkleinert und am Plattenrand vergrössert.

Im vorliegenden Bericht werden die Diagramme der Betonstauchung (Bilder 25 - 29) nicht mehr weiter verwendet. Sie wurden trotzdem in den Bericht aufgenommen, da sie Anhaltspunkte für den Schubflussverlauf im Schnitt Platte - Steg bilden (Schubfluss = differentielle Druckkraft in der Platte). Dieser Schubflussverlauf ist wichtig für eine weitere Verfeinerung des Flanschfachwerk-Modelles.

\section{4 .5 Dehnungen der Plattenarmierung}

Die für die gewählte Zielsetzung besonders wichtigen Dehnungen der Plattenarmierung sind in den Bildern 31, 32, 34, 35 und 36 dargestellt.

\section{Balken Q1 und Q2}

Die Verteilung der an der oberen und unteren Plattenarmierung gemessenen Dehnungen über die Querschnittsbreite wurde zunächst mit Hilfe des elektronischen Plotters des ETH-Rechenzentrums für alle ausgemessenen Querschnitte und Laststufen aufgezeichnet. Es zeigte sich erwartungsgemäss, dass bei der oberen Armierung die grösste Dehnung fast immer im Anschlussbereich Platte - Steg auftritt. Als Beispiel ist die Verteilung der Stahldehnung in den drei Querschnitten $A, B$ und $C$ angegeben (Bilder 31b und 32b). Der Querschnitt A liegt etwa in der Mitte zwischen Auflager und Einzellast (Bilder 31a und 32a), B bei der Einzellast und C in Balkenmitte, wo sowohl die klassische Theorie als auch das Flanschfachwerk-Modell keine Beanspruchung in der Platte liefern.

In allen drei Querschnitten $A, B$ und $C$ wurden ferner diejenigen Messstellen ausgewählt, welche bei der Höchstlast die grösste Dehnung erfuhren (Messstellen 1, 2 und 3 oben in den Bildern $31 a$ und 32a). Für diese Messstellen wurde der Verlauf der Dehnung in Abhängigkeit der Last unten auf den Bildern 31 a und 32 a aufgetragen. Dabei ist interessant festzustellen, dass bis etwa zur Anrisslast der Platte die Messstelle 3 im theoretisch unbeanspruchten Plattenbereich die grössere Dehnung aufweist. Nach dem Anreissen steigen dann aber die Dehnungen der anderen zwei Messstellen ( 1 und 2) viel schneller an, wobei Messstelle 2, welche sich unmittelbar ausserhalb der Einzellast befindet, den grösseren Wert anzeigt.

Schliesslich wurde für sämtliche ausgemessenen Querschnitte jeweils pro Armierungsstab aus den Dehnungen der sieben Messstrecken die maximale herausgesucht. Diese Maximaldehnung wird als massgebend für die Beanspruchung des betrachteten Stabes angesehen. Der Verlauf der Maximaldehnung der oberen Armierung über die Balkenlänge wurde für alle Laststufen ermittelt. Damit konnte festgestellt werden, bei welcher Laststufe die Plattenarmierung zum Fliessen kam. Beim Balken Q1 betrug diese Fliesslast $\left(P_{f A}^{E X}\right) 42.8 \mathrm{t}$, beim Balken Q2 $56.1 \mathrm{t}$. Diese zwei Lasten sind in Tabelle 7 aufgeführt.

In der Mitte der Bilder 31a und 32a ist der Verlauf der Maximaldehnung der oberen Armierung längs der Platte bei den Lasten $P_{r}=39 t$, $P_{\text {III }}=42.8 t$ und bei der Hächstlast $P_{u}$ dargestellt. 


\section{Balken Q3, Q4 und Q5}

Bei der Auswertung der Dehnungsmessungen der Plattenarmierung bei diesen drei Trägern mit Längsschub und Querbiegung wurde der Einfluss der Plattenkrümmung infolge der Querbiegung auf die Messwerte berücksichtigt (Bild 33). Die an der oberen und unteren plattenarmierung ermittelten Dehnungen $\varepsilon_{0}$ und $\varepsilon_{u}$ sind nicht in der Mittellinie des Armierungsstabes gemessen, sondern auf der Höhe der Messbolzen. Infolge der Plattenkrümmung sind $\varepsilon_{0}$ und $\varepsilon_{u}$ stets grösser als die gesuchten Stahldehnungen $\varepsilon_{\text {eo }}$ und $\varepsilon_{\text {eu }}$, welche den Kräften im Stahl entsprechen. Für verschiedene Querschnitte und Laststufen bzw. Lastkombinationen sowie Schnitte A-A wurden aus den gemessenen Dehnungen die effektiven Stahldehnungen $\varepsilon_{\text {eo }}$ und $\varepsilon_{\text {eu }}$ gerechnet. Dabei hat sich gezeigt, dass $\varepsilon_{\text {eo }}$ unabhängig von Balken, Belastung und Lage des untersuchten Schnittes etets etwa $0.9 \varepsilon_{0}$ beträgt. Zur Abschätzung von $\varepsilon_{\text {eu }}$ hingegen kann nicht ein ähnlicher Faktor eingeführt werden, da die untere Stahllage sehr nahe bei der Neutralachse des Querschnittes liegt und somit das Verhältnis $\varepsilon_{e u} / \varepsilon_{u}$ stark von der Dehnungsebene abhängt.

Aufgrund dieser Feststellung wurde die Mittelliniendehnung $\varepsilon_{\text {eo }}$ der oberen Armierung jeweils durch Multiplikation der gemessenen Werte mit dem Faktor 0.9 ermittelt. Die Dehnung $\varepsilon_{e u}$ der unteren Armierung musste hingegen - wenn benötigt - anhand der jeweiligen Dehnungsebene abgeschätzt werden.

Bei der Darstellung der Dehnungen dieser drei Balken wurde im übrigen genau gleich wie bei den Balken $Q 1$ und Q2 vorgegangen. Die Verteilung der Stahldehnung quer zur Platte in den vier Schnitten $A, B, C$ und $D$ (Schnitt $D$ wurde gegenüber Q1 und Q2 zusätzlich im Bereich reiner Biegung hinzugefügt) ist in den Bildern 34b, 35b und 36b für die wichtigsten Belastungen $\left(\bar{P}_{I}, P_{I}\right),\left(\bar{P}_{I I I}, P_{I I I}\right)$ und $\left(\bar{P}_{I I I}, P_{u}\right)$ angegeben. Dabei sind die Dehnungen der unteren Plattenarmierung nur bei der theoretischen Fliesslast ( $\bar{P}_{\text {III }}$, $P_{\text {III }}$ ) punktweise aufgetragen. Die Dehnungen der Messstellen 1, 2, 3 und 4, welche jeweils in den Schnitten $A, B, C$ und $D$ unter der Höchstlast ( $\bar{P}_{I I I}, P_{U}$ ) die grössten Werte aufwiesen, sind in den Bildern $34 \mathrm{c}, 35 \mathrm{c}$ und $36 \mathrm{c}$ in Abhängigkeit der Lasten $P, \bar{P}$ aufgezeichnet. Die Last, bei der die obere Armierung im Bereich kombinierter Beanspruchung zum Fliessen kommt, ist für die drei Balken Q3 bis Q5 in Tabelle 7 aufgeführt.

Der Verlauf der in der oberen Plattenarmierung auftretenden maximalen Dehnung über die Balkenlänge ist in den Bildern 34a, 35a und 36a angegeben. Aus diesen Bildern kann man ablesen, dass unter der Gebrauchslast $\left(\bar{P}_{I}, P_{I}\right)$, die maximale Dehnung in der Platte $1.25 \%$ beim Balken Q3 beträgt (tritt bei der Einzellast auf) bzW. $0.9 \% 0$ und $1.0 \%$ bei den Balken $Q 4$ und $Q 5$ (tritt im Bereich reiner Biegung auf). Die unter Berücksichtigung eines Verbundkoeffizienten $k=0.9$ (siehe 4.5) gerechnete entsprechende Stahlspannung beträgt $2.92 \mathrm{t} / \mathrm{cm}^{2}$ bei $Q 3 \mathrm{bzw} .2 .10 \mathrm{t} / \mathrm{cm}^{2}$ und $2.33 \mathrm{t} / \mathrm{cm}^{2}$ bei Q4 und Q5. Unter der Gebrauchslast der Platte wird damit beim Balken Q3 die rechnerische Spannung für $\bar{P}_{I}, P_{I}$ von $4.74 / 1.8=2.63 \mathrm{t} / \mathrm{cm}^{2}$ um rund $10 \%$ überschritten, während bei Q4 und Q5 die vorhandene Spannung unter dem rechnerischen Wert bleibt. 
Die in den Bildern 31, 32, 34, 35 und 36 dargestellten Stahldehnungen sind jeweils mittlere Dehnungen $\varepsilon_{\text {em }}$ über Messstrecken von $10 \mathrm{~cm}$ Länge. Da innerhalb dieser Messstrecken mit zunehmender Belastung Risse auftreten (sichtbare und Mikrorisse), ist die Dehnung im Rissquerschnitt $\varepsilon_{e}$ max grösser als die mittlere, über die ganze Messstrecke gemessene Dehnung. Für die Berechnung der Stahlspannung ist die Dehnung

$\varepsilon_{e \max }$ massgebend.

Das Verhältnis zwischen $\varepsilon_{e m}$ und $\varepsilon_{e}$ max wird als Verbundkoeffizient $k$ bezeichnet [3]. Die Grösse dieses Koeffizienten ist von vielen Faktoren abhängig (z.B. Stabdurchmesser, Oberflächenbeschaffenheit des Stahles, Belestungshähe, Länge der Messstrecke, etc.) und es liegen bis jetzt keine diesbezüglichen Versuchsresultate vor. Deshalb wurden im Zusammenhang mit der Auswertung der vorliegenden Balkenversuche zusätzliche Untersuchungen angestellt, um die Grösse von $k$ abzuschätzen. Die Resultate dieser Nebenversuche sind im Anhang zusammengefasst dargestellt. Es ergab sich, dass für die hier verwendeten Stäbe $\emptyset 6 \mathrm{~mm}$ bis zum Fliessbeginn der Armierung $\kappa$ zu 0.9 angenommen werden darf. Im Fliessbereich selbst hat $k$ keinen Einfluss mehr auf die Ermittlung der Stahlspannung.

Die in der oberen Plattenarmierung im Anschlussbereich Platte - Steg festgestellten Dehnungen (Bilder 31a, 32a, 34a, 35a und 36a) wurden mit einem Faktor von 1/0.9 vergrössert und daraus über den E-Modul die Stahlspannung und schliesslich die Kraft pro Längeneinheit des Balkens gerechnet. Die erhaltenen Diagramme sind in den Bildern 37 bis 41 wiedergegeben. Für die Balken $Q 1$ und $Q 2$ sind die Kräfte bei den drei Lasten $P_{r}, P_{I I I}$ und $P_{u}$ dargestellt, für die Balken Q3, Q4 und $Q 5$ bei den Lasten $\left(\bar{P}_{I}, P=\right.$ $1.0 \mathrm{t}),\left(\bar{P}_{I}, P_{I}\right),\left(\bar{P}_{I I I}, P_{I I}\right),\left(\bar{P}_{I I I}, P_{I I I}\right)$ und $\left(\bar{P}_{I I I}, P_{U}\right)$. In den Diagrammen ist jeweils auch der Fliesswiderstand der oberen Armierung angedeutet. Die auf den Bildern 37 - 41 aufgezeichneten experimentell ermittelten Zugkräfte in der oberen Lage der Plattenarmierung werden in Kapitel 5 diskutiert und mit den theoretischen Zugkräften nach den entsprechenden Modellen verglichen.

\subsection{Risseverhalten}

In den Bildern 43, 44, 47 und 48 sind für die zwei Balken $Q 1$ und $Q 2$ die Risse in der Platte jeweils bei den drei Lasten $P_{r}{ }^{P} \simeq P_{I I I}$ und $P_{u}$ photographisch festgehalten. Die entsprechenden Rissebilder für die Balken $Q 3$ bis $Q 5$ sind für die Laststufen ( $\bar{P}_{I}$, $\left.P_{I}\right),\left(\bar{P}_{I I I}, P_{I I I}\right)$ und $\left(\bar{P}_{I I I}, P_{U}\right)$ aus den Bildern 51,52,55, 56, 59 und 60 ersichtlich. Dabei wurden für jeden Balken die Bilder des rechten und des mittleren Plattenbereiches (zwischen den zwei Einzellasten) wiedergegeben. Auf die Wiedergabe der Rissebilder des jeweiligen linken Plattenbereiches wurde verzichtet, da die Risse im linken und im rechten plattenteil sehr ähnlich verliefen.

In den Bildern 42, 46, 50, 54 und 58 wurde für die Balken Q1 bis Q5 jeweils der Verlauf der maximalen Rissbreite längs der Platte bei den drei oben erwähnten Lasten aufgezeichnet. Ferner wurde die Breite des absolut grössten Risses in der Platte in Funktion der Belastung dargestellt. 


\section{Balken Q1 und Q2}

Die ersten Risse in der Platte entstanden bei beiden Balken, unabhängig von der plattenarmierung, am selben Ort, nämlich im Biegeschubbereich in der Nähe der Einzellast (Bilder 42 und 46). Dieser Ort deutet auf die Lage der grössten Beanspruchungen in der Platte hin. Auch die Anrisslast der Platte $\left(P_{R}\right)$ ist praktisch von der Plattenarmierung unabhängig. Sie betrug $35 \mathrm{t}$ beim Balken Q1 und $37 \mathrm{t}$ beim Balken Q2 (Tabelle 7). Das Fortschreiten der Risse in der Platte hängt jedoch stark davon ab, wie die Platte armiert ist. Wie Bild 42 zeigt, sind die Risse beim Balken Q1 (klassisches Modell) ziemlich breit. Sie nahmen bei verhältnismässig geringer Lasterhöhung sehr stark zu. Die maximale Rissbreite beträgt $0.3 \mathrm{~mm}$ bei der nächsten Laststufe nach dem Anreissen $\left(P_{r}=39 \mathrm{t}\right), 0.5 \mathrm{~mm}$ bei $P=44 \mathrm{t}\left(\underline{P_{I I I}}\right)$ und $3 \mathrm{~mm}$ bei der Höchstlast des Balkens $\left(P_{u}=51.8 \mathrm{t}\right.$ ). Demgegenüber sind die Rissbreiten beim Balken Q2 (Flanschfachwerk-Modell) bedeutend geringer (Bild 46); sie erreichen im Maximum $0.05 \mathrm{~mm}$ bei $P=45 \mathrm{t}\left(\underline{P_{I I I}}\right)$ und nur $0.3 \mathrm{~mm}$ bei der Höchstlast $\left(P_{u}=56.1 \mathrm{t}\right)$.

\section{Balken Q3, Q4 und Q5}

Ein Vergleich zwischen den Rissbreiten innerhalb dieser drei Balken (Bilder 50, 54 und 58) zeigt, dass Balken Q3 (Superposition der Armierungen aus HauptzugspannungsModell und Querbiegung) ein ungünstigeres Risseverhalten aufweist als die Balken $Q 4$ und Q5 (Q4: Superposition der Armierungen aus Flanschfachwerk-Modell und Querbiegung, Q5: Armierung für Querbiegung mit Querzugkraft aus Flanschfachwerk-Modell). Beim Balken Q3 (Bild 50) beträgt die maximale Rissbreite rund $0.2 \mathrm{~mm}$ bei der zulässigen Belastung der Platte $\left(\bar{P}_{I}, P_{I}\right), 0.5 \mathrm{~mm}$ bei theoretischer Fliesslast $\left(\bar{P}_{I I I}\right.$, $\left.P_{I I I}\right)$ *) und $1.1 \mathrm{~mm}$ bei der Höchstlast $\left(\bar{P}_{\text {III }}, P_{U}\right)$. Aus Bild 50 ist auch ersichtlich, dass bei allen Lasten die grössten Risse in der Platte im mittleren Bereich zwischen den Einzellasten auftreten, wo wenig Armierung vorhanden ist.

Bei den Balken $Q 4$ und $Q 5$ ist die maximale Rissbreite bei der zulässigen Belastung $\left(\bar{P}_{I}, P_{I}\right)$ und bei der theoretischen Fliesslast der Platte ( $\bar{P}_{I I I}$, P III) für beide Balken gleich gross, und beträgt $0.1 \mathrm{~mm}$ bzw. $0.3 \mathrm{~mm}$ (Bilder 54 und 58). Sie treten vor allem gegen die Balkenenden hin auf. Aber auch in den mittleren Bereichen beider Balken ist bei der Höchstlast die maximale Rissbreite ähnlich und beträgt $0.3 \mathrm{~mm}$. Im Auflagerbereich dagegen ist die maximale Rissbreite beim Balken Q5 etwas grösser als beim Balken Q4 $(0.4 \mathrm{~mm}$ gegenüber $0.3 \mathrm{~mm})$. Da die Platten beider Balken, Q4 und Q5, im Auflagerbereich (Bereich reiner Biegung) genau gleich armiert sind, ist dieser Unterschied von $0.1 \mathrm{~mm}$ in der maximalen Rissbreite wahrscheinlich auf die Messgenauigkeit bei den Rissbreiten (nur mit Rissemassstab gemessen) sowie andere Zufälligkeiten (in Belastung und Querschnitt) zurückzuführen.

*) Beim Balken $Q 3$ wurden bei Laststufe 12 ( $\bar{P}_{\text {III, }} P_{\text {III }}$ ) keine Riss-fotos aufgenommen. Die in Bild 50 bei ( $\bar{P}_{\text {III' }}$ ( PII ) dargesteliten Rissbreiten wurden durch Interpolation zwischen den zwei benachbarten Laststufen (11 und 13) erhalten. 


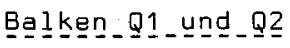

Beim Balken Q1 (klassisches Modell) bildeten sich im Anschlussbereich Platte - Steg rechts von der Einzellast (Bild 43) sehr viele Risse, deren Breite bei der Höchstlast bis $3 \mathrm{~mm}$ betrug. Mit weiterer Belastung entstand entlang der Anschlussflächen eine deutliche relative Verschiebung zwischen dem Steg und der Platte. Schliesslich trat der Bruch im Bereich rechts von der Einzellast - wo gemäss klassischem Modell sehr wenig Armierung in die Platte eingelegt wurde - durch Abscheren der Platte vom Steg auf (Bild 45), und zwar bei einer Last von ca. 50 t. Die erreichte Höchstlast $P_{u}$ betrug $51.8 \mathrm{t}$, was $92 \%$ der Biegekapazität (auf $Q 2$ beogen) entspricht.

Beim Balken Q2 (Flanschfachwerk-Modell) verhinderte die Plattenarmierung einen solchen Abscher-Bruch solange, bis ein Biegebruch in Balkenmitte auftrat (Bild 49). Die Höchstlast betrug hier 56.1 t (Tabelle 7 ).

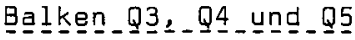

Bei den Balken Q3 und Q5 trat jeweils ein Biegebruch in Balkenmitte ein (Bilder 53 und 61). Der Bruch erfolgte durch Versagen des Betons in der Druckplatte, und zwar ausgehend von einem Querschnitt, der durch die $5 \mathrm{~cm}$ breiten Aussparungen für die Querbiege-Zugstangen geschwächt war. Beim Balken Q4 war zwei Laststufen nach Ueberschreiten der Höchstlast ebenfalls ein Biegebruch zu erwarten. Nach Laststufe 19 (siehe Tabelle 6) wurde aber noch bei konstant gehaltener Längsbelastung $P$ die Querbelastung $\bar{P}$ auf über 3 t gesteigert bis die Platte brach (Bild 57). Die Höchstlasten $\left(P_{u}\right)$ bei diesen drei Balken waren $54.2 t$ beim Balken $Q 3,56 t$ beim Balken $Q 4$ und 52.3 t beim Balken Q5 (Tabelle 7). Die dabei aufgebrachte Querbelastung war immer gleich gross und betrug $\bar{P}=\bar{P}_{\text {III }}=2.91 \mathrm{t} /$ Kolben .

Zu Balken Q5 ist zu bemerken, dass dieser bei Laststufe 14 brach (Tabelle 6), als man die Anfangs-Längsbelastung ( $\left.P_{A}=55 t\right)$ für die Dauer von zwei Minuten konstant halten wollte (Bild 20). Da für diese Laststufe keine Messungen vorliegen, wurde die Last der vorhergehenden Laststufe $P=52.3 \mathrm{t}$ als Höchstlast betrachtet. 
In diesem Kapitel werden für die fünf geprüften Balken Q1 bis Q5 die in der oberen Lage der Plattenarmierung im Anschlussbereich Platte - Steg gemessenen Kräfte, welche in den Bildern 37 bis 41 dargestellt sind, mit denjenigen aus den entsprechenden Bemessungsmodellen verglichen.

\subsection{Längsschub allein (Balken Q1 und Q2}

Für den Balken Q1 (klassisches ModeII) sind die bei den drei Lasten $P_{\Gamma}$. $P_{\text {III }}$ und $P_{u}$ $\left(P_{r}=\right.$ nächste Laststufe nach Anreissen der Platte $=39 t, P_{I I I}=$ theoretische Fliesslast der Platte $=42.8 \mathrm{t}$ und $P_{U}=$ Höchstlast im Versuch $=51.8 \mathrm{t}$ ) in der oberen Lage der Plattenarmierung experimentell ermittelten Zugkräfte in Bild 62 aufgezeichnet und den theoretischen Kräften nach dem Hauptzugspannungsmodell gegenübergestellt: Der Fliesswiderstand der oberen Armierungslage - gerechnet mit der effektiven Fliessspannung $\sigma_{f d}=4.92 \mathrm{t} / \mathrm{cm}^{2}$ - ist ebenfalls aufgetragen. Für den Balken Q2 (Flanschfachwerk-Modell) sind die entsprechenden Diagramme in Bild 63 angegeben. Die theoretischen Kräfte nach Flanschfachwerk-Modell wurden gleich wie bei der Bemessung der Plattenarmierung, $d . h$. nach $Z / 2=1 / 2 \mathrm{Q} / 4 \mathrm{y}$ berechnet. Die hier für die Darstellung verwendeten Laststufen sind $P_{I}=39 t, P_{I I I}=42.8 \mathrm{t}$ und $P_{U}=56.1 \mathrm{t}$.

Beim Balken Q1 (Bild 62) zeigt sich, dass schon bei der Laststufe nach dem ersten Riss ( $P_{I}=39 \mathrm{t}$ ) die grösste Beanspruchung im Anschlussbereich gegen die Einzellasten hin auftritt und nicht im Auflagerbereich, wo die klassische Theorie die grösste Beanspruchung liefert. Die Plattenarmierung kam bei $P_{r}=39 t$ noch nicht ganz zum Fliessen. Schon vor dem Erreichen der theoretischen Fliesslast $P_{\text {III }}=42.8 t$ floss jedoch die Plattenarmierung bereits an einigen Stellen. Mit weiterer Belastung werden die Zugkräfte nach den Auflagern, bzw. der Balkenmitte hin umgelagert, d.h. dorthin, wo im vorliegenden Balken noch unausgenützte Armierung vorhanden war. Bei der Höchstlast $P_{u}=51.8 \mathrm{t}$ fliesst die Plattenarmierung im ganzen mittleren Bereich des Balkens sowie auch über einen grösseren Bereich ausserhalb der Einzellasten. Trotzdem bleibt die starke Plattenarmierung im unmittelbaren Auflagerbereich immer noch unterbeansprucht. Es ist jedoch zu bemerken, dass die erwähnte kräfteumlagerung von grossen Verformungen der Platte begleitet ist, wodurch sehr breite Risse im Anschlussbereich entstehen. Bei $P_{u}=51.8 \mathrm{t}$ erreichte die maximale Rissbreite $3 \mathrm{~mm}$ (Bild 42). Vergleicht man für sämtliche Laststufen die theoretischen Zugkräfte nach dem Hauptzugspannungs-Modell mit den gemessenen, so stellt man durchwegs fest, dass die ersteren vor allem in den Bereichen ausserhalb und zwischen den Einzellasten erreicht bzw. überschritten wurden. Im Auflagerbereich dagegen, wo theoretisch (jedoch ohne Berücksichtigung des Einleitungsproblems der Auflagerkraft) die grössten Beanspruchungen entstehen, sind die gemessenen Kräfte wesentlich kleiner als die theoretischen. Aus Bild 62 geht auch hervor, dass im Bereich zwischen den Einzellasten, wo nach Modell keine Spannungen vorhanden wären, beachtliche Zugkräfte auftreten.

Zusammenfassend kann man folgern, dass das Hauptzugspannungs-Modell die Beanspruchungen in den Bereichen zwischen und ausserhalb der Einzellasten erheblich unterschätzt. Im Auflagerbereich hingegen ist das Gegenteil der Fall.

Beim Balken Q2 (Bild 63) ist zu erkennen, dass die maximalen gemessenen Zugkräfte in 
der Plattenarmierung bei allen Belastungen ( $P_{r}, P_{\text {III }}$ und $P_{u}$ ) knapp ausserhalb der Einzellast auftreten. Diese Stellen mit den grössten Zugkräften liegen etwa in der Mitte des Bereiches, wo nach Fachwerk-Modell die Zugkräfte entstehen. Im Bruchzustand $P_{U}=56.1 \mathrm{t}$ erreichte die maximal gemessene Zugkraft an nur zwei Stełlen die Fliesskraft der Plattenarmierung, so dass keine Umlagerung der Zugkräfte in der Platte infolge Fliessen, wie bei $Q 1$, stattfand. Aus Bild 63 ist ferner ersichtlich, dass die gemessenen Zugkräfte bei $P_{r}=39 t$ sowie bei $P_{I I I}=42.8 \mathrm{t}$ klein sind gegenüber den theoretischen Werten. Die Erklärung dafür ist, dass die Platte bei $\mathrm{P}_{r}=$ $42.8 \mathrm{t}$ nur einige wenige und sehr feine Risse (Breite $\leq 0.05 \mathrm{~mm}$ ) in den Bereichen ausserhalb der Einzellasten aufwies, so dass die Zugkräfte im Anschlussbereich Platte - Steg grösstenteils immer noch vom Beton aufgenommen wurden. Das Fachwerk-Modell bildete sich erst nach erheblicher Rissebildung, d.h. hier im Bereiche der theoretischen Fliesslast, aus. Unter $P_{u}$ betrugen dann die gemessenen Zugkräfte pro Längeneinheit im Maximum immerhin etwa $80 \%$ der theoretischen Kräfte nach FlanschfachwerkModell gemäss der Gleichung $Z=Q / 4 y$. Eine bessere Uebereinstimmung mit den gemessenen Maximalwerten würde sich bei der Berechnung der theoretischen Kräfte nach der den Einfluss der Stegbreite berücksichtigenden Formel

$$
z=\frac{Q}{4 y} \cdot \frac{b-b}{b}
$$

ergeben.

Aus Bild 63 ist ferner ersichtlich, dass in der platte auch ausserhalb des theoretisch beanspruchten Bereiches Querzugkräfte auftreten. Im Auflagerbereich und in Balkenmitte wurden also - im Widerspruch zum Modell - Zugkräfte gemessen, was auf die "Verträglichkeitswirkung" der beiden Plattenhälften zurückzuführen ist.

Bei der Interpretation dieses Versuches ist unbedingt zu beachten, dass ausserhalb der gemäss Fachwerk-Modell beanspruchten Bereiche die gleiche (konstruktive) Armierung wie innerhalb dieser Bereiche eingelegt war $\left(\mu=\mu{ }^{\prime}=0.26 \%\right)$. Wäre ausserhalb der theoretisch notwendigen Armierung eine geringere konstruktive Armierung vorhanden gewesen, so wäre die Beanspruchung der Armierung innerhalb des theoretischen Querzugbereiches zweifellos höher ausgefallen.

\subsection{Längsschub mit Querbiegung (Balken Q3, Q4 und Q5)}

Für diese drei Balken sind die in der oberen Lage der Plattenarmierung experimentell ermittelten Zugkräfte in den Bildern 64 bis 66 aufgetragen und mit den theoretischen Zugkräften - nach dem entsprechenden Bemessungsmodell gerechnet - verglichen. Der Vergleich erfolgte bei den drei Lastkombinationen $\bar{P}_{I}, P_{I}=$ zulässige Lasten der Platte, $\bar{P}_{\text {III' }}$ III $=$ theoretische Fliesslast der Platte und $\bar{P}_{\text {III, }} P_{U}=$ Höchstlasten im Versuch. In diesen Bildern ist jeweils auch der Fliesswiderstand der oberen Armierungslage angegeben.

Die dargestellten theoretischen Zugkräfte wurden folgendermassen berechnet: In den Bereichen, wo kein Längsschub auftritt (Bereich reiner Biegung) wurde die Zugkraft $K_{0}$ in der oberen Plattenarmierung durch die Formel $K_{0}=M_{q} / y_{p}$ ermittelt. Der Hebelarm $y_{p}$ der inneren Kräfte in der Platte wurde aus der Dehnungsebene des Plattenquerschnittes bei Fliessbeginn des Stahls zu $\mathrm{y}_{p}=8.1 \mathrm{~cm}$ gerechnet. Für kleinere Beanspruchungen kann der gleiche Wert verwendet werden. Bei den Balken Q3 und Q4 wurden 
in den Bereichen mit kombinierter Beanspruchung zu dieser Zugkraft aus Querbiegung hinzu noch die Zugkräfte aus Längsschub (für Balken Q3 nach Hauptzugspannungs-Modell und für Balken Q̀4 nach Flanschfachwerk-Modell gemäss $Z / 2=1 / 2 \cdot Q / 4 y$ gerechnet) addiert. Beim Balken $Q 5$ wurden die im Bereich kombinierter Beanspruchung am Plattenquerschnitt wirkenden äusseren kräfte $M_{Q}$ und $Z=Q / 4 y$ bestimmt. Die inneren kräfte im Beton und Stahl wurden unter der Annahme von Ebenbleiben der Querschnitte so errechnet, dass sie den jeweiligen äusseren Kräften äquivalent sind.

Beim Balken Q3 (Superposition der Armierungen aus Hauptzugspannungs-Modell und Querbiegung) zeigt sich (Bild 64), dass theoretisch die Beanspruchung im Bereich der Auflager bei allen Belastungen überschätzt wird. Auch in den Plattenbereichen ausserhalb der Auflager (reine Biegung) werden die theoretischen Kräfte nicht erreicht. Besonders bei höheren Laststufen ist dies darauf zurückzuführen, dass die Platte über dem Auflager infolge der dortigen starken Armierung ziemlich steif ist und somit einen Teil der Querbiegemomente an sich zieht, wodurch die Bereiche ausserhalb der Auflager entlastet werden. Unter zulässiger Belastung kommt noch folgender Effekt hinzu: die Risse sind noch nicht vollständig ausgebildet und der Beton trägt noch einen Teil der Zugkraft; die am Stahl gemessenen effektiven Kräfte werden dadurch kleiner. Im Bereich zwischen den zwei Einzellasten stimmen bei den zulässigen Lasten $\left(\bar{P}_{I}\right.$, $\left.P_{I}\right)$ die gemessenen und die theoretischen Zugkräfte noch einigermassen überein. Bereits unter $\bar{P}_{\text {III }}=2.91 \mathrm{t}$ und $\mathrm{P}_{\text {II }}=33.2 \mathrm{t}$ (Bild 39) fliesst jedoch der ganze Bereich zwischen den zwei Lasten und auch ein kurzer Bereich ausserhalb dieser Lasten. Mit weiterer Belastung schreitet das Fliessen der Plattenarmierung gegen die Balkenenden weiter fort, hört aber vor dem Auflagerbereich auf. In diesem Bereich kommt die Plattenarmierung auch unter den Bruchlasten ( $\bar{P}_{\text {III' }} P_{u}$ ) nicht ins Fliessen.

Bei den Balken Q4 und Q5 (Q4: Superposition der Armierungen aus Flanschfachwerk-Modell und Querbiegung; Q5: Armierung für Querbiegung mit Querzugkraft aus FlanschfachwerkModell) sind die oberen Plattenarmierungen sowohl bezüglich Lage als auch bezüglich Stärke praktisch gleich, die unteren Armierungen hingegen verschieden. Daher verlaufen die in der oberen Armierung gemessenen Zugkräfte bei diesen zwei Balken sehr ähnlich. Bei den zulässigen Lasten ( $\left.\bar{P}_{I}, P_{I}\right)$ liegen die gemessenen Zugkräfte im allgemeinen noch wesentlich tiefer als die theoretischen (Bilder 65 und 66). Dies ist wiederum wie beim Balken Q3 darauf zurückzuführen. dass der Beton noch nicht überall voll durchgerissen ist und daher einen Teil der Zugkräfte übertragen kann. Unter den theoretischen Fliesslasten $\bar{P}_{I I I}$, P III nähern sich indessen die gemessenen Zugkräfte den theoretischen. Die Plattenarmierung kommt aber nur in den Auflagerbereichen (bei Q5 auch in Balkenmitte), wo nach den Modellen lediglich Querbiegemomente wirken, ins Fliessen. Dies kann wiederum mit der "Verträglichkeitswirkung" der Plattenhälften er-

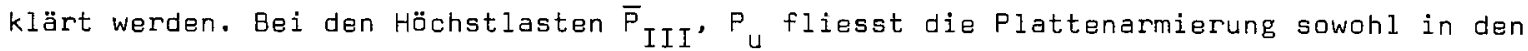
Bereichen mit reiner Biegung als auch in den Bereichen mit Längsschub und Querbiegung, und es ergibt sich vor allem beim Balken Q5 eine gute Uebereinstimmung zwischen den gemessenen und den theoretischen Zugkräften in der Platte. Diese gute Uebereinstimmung deutet auf die Zweckmässigkeit der beiden Bemessungsmodelle hin. Das Modell "Armierung für Querbiegung mit Querzugkraft aus Flanschfachwerk-Modell" (Balken Q5) erscheint grundsätzlich richtiger, da es je nach den massgebenden Schnittkraftkombinationen Längsschub/Querbiegung zu Ersparnissen in der unteren Armierungslage führen kann. Das Modell "Superposition der Armierungen aus Flanschfachwerk-Modell und aus Querbiegung" führt jedach zum gleichen Resultat, sofern der gleiche innere Hebelarm y vorausgesetzt und die untere Zugkraft aus dem Flanschfachwerk-Modell mit der Druckkraft aus Querbiegung "verrechnet" wird (vgl. [2]). 
Beim Vergleich zwischen Versuchsergebnissen und Modellen der gemäss Fachwerk-Modell armierten Balken Q4 und $Q 5$ hat sich gezeigt (siehe 5.2), dass nicht nur im Gebrauchslastbereich, sondern - in geringerem Masse - auch im Bereich der Fliesslasten die theoretischen Werte über den gemessenen liegen. Diese Feststellung gilt auch noch dann, wenn die theoretischen Werte unter Berücksichtigung des Einflusses der Stegbreite gemäss Gleichung ( 8 ) berechnet werden, sofern man die Mittelwerte über den Bereich "Kombinierte Beanspruchung" und nicht die Maximalwerte in Betracht zieht. In den folgenden beiden Abschnitten werden hiezu mögliche Erklärungen angeführt.

\subsubsection{Ausbreitwinkel}

Der "tatsächliche" Ausbreitwinkel B im Flanschfachwerk ist kleiner als der angenommene Wert von arc $\mathrm{tg}$ 0.5. Mit zunehmender Plastifizierung der Querarmierung verringert er sich. Ein plausibler Wert ist beispielsweise arc tg 0.4 . Er führt auf die Bemessungsformel

$$
z=\frac{Q}{5 y} \cdot \frac{b-b_{0}}{b} .
$$

\subsubsection{Interaktion Biegung - Längsschub}

Die Querzugkraft $Z$ infolge Längsschub verlagert sich mit zunehmender Beanspruchung aus der Plattenmitte (bzw. aus dem Schwerpunkt der Längsbiegedruckkraft) gegen die Querbiegedruckzone hin. Dadurch wird die obere Längsschubarmierung entlastet.

Aus den beschriebenen Versuchen können bezüglich dieser beiden Effekte keine endgüItigen Schlüsse gezogen werden. Für die praktische Bemessung der Längsschubarmierung erscheint es jedoch möglich, unter den im folgenden Abschnitt angegebenen Voraussetzungen die Formel (9) zu verwenden.

\subsection{Obere Schubspannungsgrenze}

Die sogenannte obere Schubspannungsgrenze entspricht der Schubspannung, bei der die Schubarmierung "gleichzeitig" mit dem Bruch des Betons ins Fliessen kommt. Liegt die tatsächliche Schubspannung oberhalb dieser Grenze, so versagt der Beton - insbesondere infolge schiefem Druck - bevor die Schubarmierung fliesst, d.h. voll ausgenützt werden kann.

Die obere Schubspannungsgrenze ist in praktisch allen Normen in der einen oder andern Form angegeben. Hiermit wird ein duktiles Verhalten im Bruchzustand angestrebt, d.h. es sollen Veberarmierungen vermieden bzw. Sprödbrüche ausgeschlossen werden. Die obere Schubspannungsgrenze ist massgebend für die erforderlichen Betanmindestabmessungen.

Die vorliegenden Versuche waren nicht darauf angelegt, die Lage der oberen Schubspannungsgrenze in Druckplatten mit Querbiegung ermitteln zu können. Es ist zu ver- 
muten, dass diese Grenze infolge der Schwächung der Platte durch Biegerisse erheblich tiefer liegt als ohne Querbiegung. Bei den Versuchen [7] wurde in Stegen eine solche Abminderung der oberen Schubspannungsgrenze durch gleichzeitig wirkende Querbiegung eindeutig festgestellt. In den hier beschriebenen Versuchen betrugen die bei den stets nach Fliessen der Schubarmierung eingetretenen Brüchen erreichten nominellen Längsschubspannungen in der platte bis $34.2 \mathrm{~kg} / \mathrm{cm}^{2} .56 \mathrm{t} / 42.8 \mathrm{t}=45 \mathrm{~kg} / \mathrm{cm}^{2}(\mathrm{vgl}$. Tabelle 7). Dies entspricht knapp $80 \%$ der in der Norm SIA 162 angegebenen und bereits mit einer gewissen Sicherheit festgelegten oberen Schubspannungsgrenze ( $f u ̈ r$ Q2 und Q4 $B_{W}=360 \mathrm{~kg} / \mathrm{cm}^{2}: \tau^{*}=5 \tau \tau_{1}^{*}=5.11 .2 \mathrm{~kg} / \mathrm{cm}^{2}=56 \mathrm{~kg} / \mathrm{cm}^{2}$ nach Richtlinie 17 bzw. $\tau^{\prime} \cdot 1.3=2.5 \sqrt{ } \beta_{w} \cdot 1.3=62 \mathrm{~kg} / \mathrm{cm}^{2}$ nach Art. 5.13). Aufgrund dessen und gestützt auf die - allerdings nicht direkt übertragbaren - Ergebnisse der Untersuchungen [7] ist zu empfehlen, vorläufig und bis weitere Forschungsergebnisse vorliegen, die obere Schubspannungsgrenze in Druckplatten auf rund $75 \%$ des Wertes ohne Querbiegung abzumindern. 
Aus den vorliegenden Versuchsresultaten können die folgenden Schlüsse gezogen und vorläufigen Empfehlungen gegeben werden:

\section{Längsschub allein}

Es wird vorausgesetzt, dass durch vorangegangene Querbiegung, Zwängungen aus Schwinden. Temperatur, usw. bereits gewisse Risse vorhanden sind. Eine Wirkung der Betonzugfestigkeit wird somit nicht berücksichtigt.

1. Die Bemessung der Plattenquerarmierung aufgrund der Hauptzugspannungen aus der einfachen Balkentheorie führt zu falschen Ergebnissen. Querschitte können erheblich sowohl unter- als auch überbemessen werden, und insgesamt wird die Armierung längs eines Trägers am falschen Ort eingelegt.

2. Ein besseres und zudem einfacheres Bemessungsmodell ist das Flanschfachwerk-Modell (Bild 4). Sofern nicht einzelne Stäbe der Plattenarmierung vor dem Erreichen der Biegekapazität bzw. Stegschubkapazität des betrachteten Trägers ins Fliessen kommen sollen, kann die pro Längeneinheit im Anschlussquerschnitt Platte - Steg erforderliche Querarmierung nach der Formel

$$
z=\frac{Q}{4 y} \cdot \frac{b-b a}{b}
$$

bemessen werden. Diese entspricht einem Ausbreitwinkel der Druckkräfte in der Platte von arc $\operatorname{tg}=0.5$.

Wird ein vorzeitiges Fliessen einzelner Armierungsstäbe in Kauf genommen, so kann die Querarmierung nach der Formel

$$
z=\frac{Q}{5 y} \cdot \frac{b-b o}{b}
$$

bestimmt werden. Dies entspricht einem Ausbreitwinkel von arc tg 0.4. Hiermit dürfte - sofern überall eine Mindestarmierung gemäss 5 . eingelegt ist - noch eine genügende Sicherheit sowohl gegen vorzeitiges Abscheren der Platte vom Steg als auch gegen unzulässige Rissbreiten im Gebrauchszustand gewährleistet sein. Bei einer weiteren Reduktion des Bemessungswinkels würde jedoch - übliche Verhältnisse vorausgesetzt - vor allem die Rissebeschränkung wesentliche Probleme aufwerfen.

3. Gegenüber dem Trägerquerschnitt mit der Querkraft $Q$ ist die Plattenquerarmierung mit einem Versatzmass in Richtung des zunehmenden Momentes von

$$
v=\frac{y}{2 \operatorname{tg} \alpha}+\frac{b}{4 \operatorname{tg} \beta}
$$

anzuordnen. Als praktischer Wert kann

$$
v=0.6(y+b)
$$

angenommen werden. 
4. Die Zugkraft $Z$ gemäss 2. ist grundsätzlich als auf der Höhe des Schwerpunktes der Plattendruckkraft wirkend anzunehmen. Im allgemeinen kann die erforderliche Querarmierung jedoch in zwei gleichen Lagen oben und unten in der Platte angeordnet werden.

5. Die Anwendung der vorstehend gegebenen Regeln setzt voraus, dass längs des ganzen Balkens in der Druckplatte je oben und unten eine konstruktive Mindestarmierung von etwa $0.10-0.15 \%$ vorhanden ist.

6. Die obere Schubspannungsgrenze kann gleich wie in Stegen angesetzt werden.

\section{Längsschub mit Quertiegung}

7. Die Superposition der Armierungen aufgrund der Querbiegung und der Hauptzugspannungen aus der einfachen Balkentheorie führt zu falschen Resultaten. Es gelten die unter 1 . gemachten Feststellungen sinngemäss.

8. Die erforderliche Querarmierung kann durch Superposition der Querbiegearmierung und der Längsschubarmierung nach Flanschfachwerk-Modell gemäss 2., 3. und 4. bestimmt werden. Sofern es die vorhandenen Schnittkraftkombinationen Längsschub/ Querbiegung erlauben (z.B. ständige Querbiegung), kann auf der Biegedruckseite die Längsschubarmierung entsprechend der Wirkung der Biegedruckkraft, jedoch höchstens bis auf die Mindestarmierung gemäss 5 ., vermindert werden.

9. Die obere Schubspannungsgrenze ist aufgrund der Schwächung des Plattenquerschnittes durch Biegerisse und bis eingehendere Versuche zu dieser Frage vorliegen, auf rund $75 \%$ des Wertes ohne Querbiegung abzumindern.

\section{Einschränkungen}

Die obigen Folgerungen sind als vorläufig zu betrachten. Sie müssen in Teilaspekten (z.B. Interaktion Längsschub/Querbiegung im Bruchzustand) noch durch weitere Untersuchungen ergänzt werden. Die Empfehlungen gelten nur für bezüglich Geometrie und Belastung symmetrische T-Querschnitte. Modelle und Empfehlungen für andere Querschnitte sind in Vorbereitung.

Die Ergebnisse sind ferner nur für Druckplatten gültig. Wie erste Versuche gezeigt haben, liegen die Verhältnisse in Zugplatten vor allem bezüglich Ausbreitwinkel wesentlich ungünstiger. 
In Druckplatten von dünnwandigen Plattenbalken- und Hohlkastenquerschnitten treten häufig die Beanspruchungen Längsschub und Querbiegung auf. Zur Erforschung des Verformungs- und Tragverhaltens solcher Druckplatten wurden am Institut für Baustatik und Konstruktion der Eidgenässischen Technischen Hochschule Zürich Versuche an fünf Stahlbetonträgern durchgeführt. Es wurden verschiedene Bemessungsmodelle überprüft und anhand der Versuchsresultate verglichen. In diesem Bericht werden die Ergebnisse der Untersuchungen und vorläufige Empfehlungen für die Bemessung dargelegt.

An zwei Trägern (Q1, Q2) wurde das Problem "Längsschub allein" erforscht (siehe Bild 18). Bei drei Trägern (Q3, Q4 und Q5) wurde die Beanspruchung "Längsschub und Querbiegung" untersucht (Bild 19). Diese Träger waren schlaff armiert. Später wurden noch zwei weitere Träger (Q6, Q7) geprüft, deren Druckplatte eine teilweise Quervorspannung aufwies. Hierüber wird jedoch in einer separaten Publikation [2] berichtet.

Der Bemessung der Plattenquerarmierung wurden die folgenden Modelle zugrunde gelegt:

\section{Längsschub allein:}

Träger Q1: Aufnahme der Hauptzugspannungen aus der einfachen Balkentheorie (Bild 2)

Träger Q2: Aufnahme der Querzugkräfte aus einem Flanschfachwerk-Modell (Bild 4)

\section{Längsschub und Querbiegung:}

Träger Q3: Superposition der Armierungen aus Hauptzugspannungs-Modell und aus Querbiegung (Bild $7 a$ )

Träger Q4: Superposition der Armierungen aus Flanschfachwerk-Modell und aus Querbiegung (Bild 7b)

Träger Q5: Armierung für Querbiegung mit Querzugkraft aus Flanschfachwerk-Modell (Bild 7o)

Alle übrigen Parameter, wie Trägerabmessungen, Biege- und Querkraftarmierungen des Steges, Betonqualität, usw., wurden konstant gehalten (Bild 10).

Aus den Versuchsresultaten können die folgenden Schlüsse gezogen und vorläufigen Empfehlungen gegeben werden:

\section{Längsschub allein}

1. Die Bemessung der Plattenquerarmierung aufgrund der rechnerischen Hauptzugspannungen führt zu falschen Ergebnissen.

2. Die pro Längeneinheit des Balkens erforderliche Querarmierung kann zweckmässigerweise nach der Formel

$$
z=\frac{Q}{4 y} \cdot \frac{b-b}{b}
$$

gemäss Fachwerk-Modell bemessen werden. Dies entspricht einem Ausbreitwinkel in der Platte von arc tg 0.5 .

Sofern ein Fliessen einzelner Armierungsstäbe vor dem Erreichen der Biegekapazität bzw. Stegschubkapazität des betrachteten Trägers in Kauf genommen wird, kann ein 
Ausbreitwinkel von arc $\mathrm{tg} 0.4$ angesetzt werden. Dem entspricht eine Bemessung der Querarmierung nach der Formel

$$
z=\frac{Q}{5 y} \frac{b-b}{b} \text {. }
$$

3. Gegenüber dem Trägerquerschnitt mit der Querkraft Q ist die Plattenquerarmierung um das Versatzmass

$$
v=0.6(y+b)
$$

in Richtung des zunehmenden Momentes zu verschieben.

4. Die gemäss Fachwerk-Modell erforderliche Querarmierung kann im allgemeinen in zwei gleichen Lagen oben und unten in der Platte eingelegt werden.

5. Längs des ganzen Balkens ist in der Druckplatte je oben und unten eine konstruktive Mindestarmierung von etwa $0.10-0.15 \%$ anzuordnen.

6. Die obere Schubspannungsgrenze kann gleich wie in Stegen angesetzt werden.

\section{Längsschub und Querbiegung}

7. Die Superposition der Armierungen aus den Hauptzugspannungen und aus Querbiegung führt zu falschen Resultaten.

8. Die erforderliche Querarmierung kann durch Superposition der Querbiegearmierung und der Längsschubarmierung gemäss Fachwerk-Modell bestimmt werden. Auf der Biegedruckseite kann die Längsschubarmierung entsprechend der gleichzeitig wirkenden Biegedruckkraft abgemindert werden.

9. Die obere Schubspannungsgrenze ist auf rund $75 \%$ des Wertes ohne Querbiegung abzumindern.

Die Ergebnisse und Empfehlungen gelten nur für Druckplatten. Wie erste Versuche gezeigt haben. Iiegen die Verhältnisse in Zugplatten vor allem bezüglich Ausbreitwinkel wesentlich ungünstiger. 
Dans les dalles minces comprimées des profils à Te et des sections évidées interviennent fréquement des sollicitations combinées d'effort tranchant longitudinal et de flexion transversale. Afin de contribuer à la détermination de la capacité portante et de la déformation de telles dalles, cinq essais de profils en béton armé furent exécutés à l'école polytechnique de Zurich. Divers modèles permettant le dimensionnement furent examinés et comparés à l'aide d'essais. Ce rapport expose les résultats de ces investigations et donne des recommandations provisoires pour le dimensionnement.

Deux poutres (Q1, Q2) furent chargées de manière à examiner séparément le comportement à l'effort tranchant longitudinal (Fig. 18). Les trois autres (Q3, Q4, Q5) furent sollicitées simultanément à l'effort tranchant longitudinal et à la flexion transversale. L'armature de ces cinq poutres étaient uniquement constituée d'acier d'armature conventionnelle. Par la suite, deux autres poutres (Q6, Q7) dont la dalle de compression était partiellement précontrainte perpendiculairement à l'axe de la pièce furent encore soumis à des essais. Le compte-rendu de ces derniers essais ne fait pas partie du rapport ci-joint, mais fera l'objet d'une prochaine publication [2] .

Les cinq modèles exposés ci-dessous ont servis de base au dimensionnement de l'armature transversale.

\section{Effort tranchant longitudinal isolé}

Poutre Q1:

Reprise des contraintes principales de traction obliques calculées d'après la théorie classique de la résistance des matériaux (Fig. 2).

Poutre Q2:

Reprise de la traction transversale, déterminée à partir d'un système triangulé (Fig. 4).

\section{Effort tranchant longitudinal et flexion transversale}

Poutre Q3:

Superposition des armatures nécessaires à la reprise des contraintes de traction obliques et de la flexion transversale (Fig. 7a).

Poutre Q4:

Superposition des armatures issues du système triangulé et de la flexion transversale (Fig. 7b).

\section{Poutre 5:}

Dimensionnement de l'armature transversale à la flexion accompagné de traction. La traction est issue de l'influence de l'effort tranchant longitudinal sur le système triangulée.

Tous les autres paramètres, tels que qualité du béton, dimensions de la pièce, armatures de l'âme, etc. pouvant influencer les résultats furent maintenus constants. 
Ces différents essais et l'examen de leurs résultats permettent de tirer et d'émettre les conclusions respectivement recommandations suivantes:

\section{Effort tranchant longitudinal isolé}

1. Le dimensionnement de l'armature transversale selon la théorie classique de la résistance des matériaux conduit à de faux résultats.

2. L'armature transversale nécessaire par unité de longueur de la poutre peut être déterminée de manière satisfaisante par la formule

$$
z=\frac{Q}{4 y} \cdot \frac{b-b_{0}}{b} \text {. }
$$

Cette relation exprime la traction transversale du système triangulé, en tenant compte d'une repartition transversale des efforts spécifiques sous un angle équivalent à arc tg 0.5 . Pour le cas, où l'on accepte dans quelques barres un écoulement avant que l'on atteigne la capacité portante à la flexion ou à l'effort longitudinal, on pourra introduire un angle de répartition transversale égal à arc $\operatorname{tg} 0.4$. La formule prendra alors la forme suivante:

$$
z=\frac{Q}{5 y} \cdot \frac{b-b_{o}}{b} \text {. }
$$

3. L'armature transversale sera par rapport à l'armature d'effort tranchant d'une même section translatée dans la direction croissante des moments de flexion d'une quantité égale à: $v=0.6(y+b)$.

4. L'armature déterminée à l'aide du système triangulé peut être répartie dans la nappe inférieure et supérieure.

5. L'armature minimale transversale de la dalle comprimée sera au moins égale à $0.10-0.15 \%$ pour chacune des deux nappes.

6. La contrainte tangentielle limite de la dalle peut être admise égale à celle de 1 'âme.

\section{Effort tranchant longitudinal et flexion transversale}

7. La superposition des armatures déterminées à l'aide de la théorie classique de la résistance des matériaux et de celle nécessaire à la reprise de la flexion transversale conduit à de faux résultats.

8. L'armature transversale peut être déterminée de manière satisfaisante en superposant l'armature de flexion transversale à celle de l'effort tranchant longitudinal issue du système triangulé. Une réduction de l'armature d'effort tranchant est permise dans la partie comprimée de la dalle sous l'influence de la flexion transversale.

9. La contrainte tangentielle limite sera fixée à $75 \%$ de la valeur correspondante sous flexion transversale.

Ces résultats et recommandations ne sont valables que pour les dalles comprimées. Comme les premiers essais l'ont montrés, les rapports dans les dalles de tractions, en particulier pour l'angle de répartition transversale, sont moins favorables. 
In the compression flanges of T-section and box-section members, the actions of longitudinal shear and transverse bending often occur. To investigate the deformation and load behavior of such compression flanges, tests on five reinforced concrete beams were carried out at the Swiss Federal Institute of Technology Zürich. Different design models were tested and the results compared. The results of these tests and preliminary recommendations for design are presented in this report.

In two beams (Q1, Q2) the problem of "longitudinal shear alone" was examined (see fig. 18). The action of "longitudinal shear and transverse bending" was investigated with three beams (Q3, Q4 and Q5 - fig. 19). These beams were reinforced with conventional reinforcement. Later two further beams were tested (Q6, Q7). The compression flanges of these beams were transversely partially prestressed. This however is reported in a separate publication [2].

The design of the transverse flange reinforcement was based upon the following models: Longitudinal Shear alone

Beam Q1: The taking up of main tensile stresses from simple beam theory (fig. 2). Beam Q2: Taking up of transverse tensile forces from a flange truss model (fig. 4).

Beam Q3: Superposition of reinforcement required from main tensile stress model and for transverse bending ( $f i g$. $7 a$ ).

Beam Q4: Superposition of reinforcement from flange truss model and for transverse bending ( $f i g .7 b)$.

Beam Q5: Reinforcement for transverse bending with transverse tensile force from flange truss model (Fig. 7c).

All other parameters such as beam dimensions, bending and shear reinforcement of the web, concrete quality etc. were held constant (fig. 10).

From the test results the following conclusions can be drawn and preliminary recommendations given:

\section{Longitudinal Shear alone}

1. The design of the slab transverse reinforcement based upon calculated main tensile stresses leads to wrong results.

2. The required transverse reinforcement per unit length of the beam can be appropriately designed with the formula

$$
z=\frac{Q}{4 y} \cdot \frac{b-b_{D}}{b}
$$

according to the truss model. This corresponds to an angle of inclination of the compression diagonals in the flange of arc tan 0.5 .

If the case of a single reinforcement bar yielding before the beam reaches its flexural capacity or web shear capacity is accepted, then an angle of arc tan 0.4 
for the flange compression diagonals can be applied. This corresponds to a design of the transverse reinforcement with the formula

$$
z=\frac{Q}{5 y} \cdot \frac{b-b}{b}
$$

3. The transverse reinforcement of the compression flange for a beam cross-section with shear force $Q$ is displaced by a distance $v=0.6(y+b)$ in the direction of the increasing moment.

4. The required transverse reinforcement according to the truss model can be in general laid in two equal positions, top and bottom, in the flange.

5. A minimum transverse reinforcement of 10 - $15 \%$ should be placed top and bottom in the flange compression flange.

6. The same maximum permissible shear stress as for the web can be used.

Longitudinal Shear and Transverse Bending

7. The superposition of reinforcement for main tensile stress and for transverse bending leads to wrong results.

8. The required transverse reinforcement can be determinated by superposition of the transverse bending reinforcement and the londitudinal shear reinforcement according to the truss model. On the bending compressive face the longitudinal shear reinforcement can be reduced corresponding to the simultaneous bending compressive force.

9. The maximum permissible shear stress is reduced to about $75 \%$ of the value without transverse bending.

The results and recommendations are valid only for compression flanges. First Tests have shown that for each different angle of inclination of the force diagonals the behavior of tension flange is less favourable. 
Der vorliegende Versuchsbericht wurde im Rahmen der zweiten Phase des forschungsprogrammes "Teilweise Vorspannung im Brückenbau" ausgearbeitet. Dieses Programm wird aufgrund eines Beitrages aus dem

"Kredit zur Förderung der Strassenbauforschung"

des Eidgenössischen Departementes des Innern durchgeführt. Für diese grosszügige Unterstützung danken die Verfasser dem Departement des Innern und der Kommission für Strassenbauforschung mit ihrem Präsidenten. Herrn Dr. R. Ruckli, herzlich.

Für die Ueberlassung von Armierungsstählen und Komponenten der Belastungseinrichtung sind die Verfasser den folgenden Firmen zu aufrichtigem Dank verpflichtet:

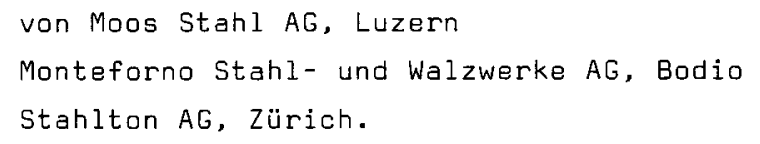

Der experimentelle Teil der Untersuchungen konnte an der Eidgenössischen Materialprüfungs- und Versuchsanstalt (EMPA) in Dübendorf durchgeführt werden. Für dieses Entgegenkommen und die stets gute Zusammenarbeit möchten die Verfasser Herrn Prof. Dr. Th. Erismann, Direktionspräsident, und Herrn Dr. M. Ladner, Vorsteher der Abteilung Massivbau, bestens danken.

Bei der Versuchsdurchführung und der Gestaltung dieses Berichtes haben vor allem die Herren K, Bucher, L. Sieger sowie Frl. S. Burki mitgewirkt. Herr Dipl.Ing. M. Baumann hat die messtechnischen Aufgaben betreut. Herr Dipl. Ing. A. Bacchetta war bei der Vorbereitung der Versuche sowie bei deren Durchführung beteiligt. Für diese Unterstützung sprechen die Verfasser den Genannten ihren herzlichen Dank aus. 


\section{LITERATURVERZE ICHNIS}

[1] Mörsch E.: "Der Eisenbetonbau, seine Theorie und Anwendung". 6. Auflage, 1. Band, II. Hälfte, Seite 260, Stuttgart 1929, Verlag Konrad Wittwer.

[2] Bachetta A., Bachmann H.: "Versuche zur teilweisen Vorspannung für Längsschub und Querbiegung in Druckplatten von Betonträgern". Institut für Baustatik und Konstruktion, ETH Zürich, Bericht Nr. 6504-9, 1977. Birkhäuser Verlag Basel und stuttgart.

[3] Bachmann H.: "Stahlbeton I und II". Vorlesungen an der Abt. für Bauingenieurwesen der ETH Zürich. 2. Auflage 1974/75.

[4] Lampert P., Wegmüller A., Thürlimann B.: "Einfluss der Dehngeschwindigkeit auf Festigkeitswerte von Armierungsstählen". Institut für Baustatik. ETH Zürich, Bericht Nr. 10, April 1967, Birkhäuser Verlag Basel und Stuttgart.

[5] Krauss R., Bachmann H.: "Biege- und Schubversuche an teilweise vorgespannten Leichtbetonbalken". Institut für Baustatik, ETH Zürich, Bericht Nr. 6504-4, Oktober 1971, Birkhäuser Verlag Basel und Stuttgart.

[6] Norm SIA 162 (1968): "Norm für die Berechnung, Konstruktion und Ausführung von Bauwerken aus Beton. Stahlbeton und Spannbeton". Schweizerischer Ingenieurund Architekten-Verein, Zürich.

[7] Kaufmann J., Menn C.: "Versuche über Schub bei Querbiegung". Institut für Baustatik und Konstruktion, ETH Zürich, Bericht Nr. 7201-1, Dezember 1976, Birkhäuser Verlag Basel und Stuttgart. 


\begin{tabular}{|c|c|}
\hline$H$ & Trägerhöhe \\
\hline h & statische Höhe \\
\hline$y, y_{p}$ & Hebelarme \\
\hline$b$ & Plattenbreite \\
\hline$b_{0}$ & Stegbreite \\
\hline d & Plattendicke \\
\hline$I_{i}$ & ideelles Trägheitsmoment \\
\hline $\mathrm{s}_{i}$ & ideelles statisches Moment \\
\hline a & Schubspannweite \\
\hline$F_{e}$ & Plattenquerarmierung \\
\hline $\mathrm{F}_{\mathrm{eB}}$ & Bügelarmierung \\
\hline $\mathrm{F}_{\text {eL }}$ & Längsarmierung \\
\hline$t$ & Abstand \\
\hline$\emptyset$ & Durchmesser \\
\hline
\end{tabular}

\section{Kraftgrössen}

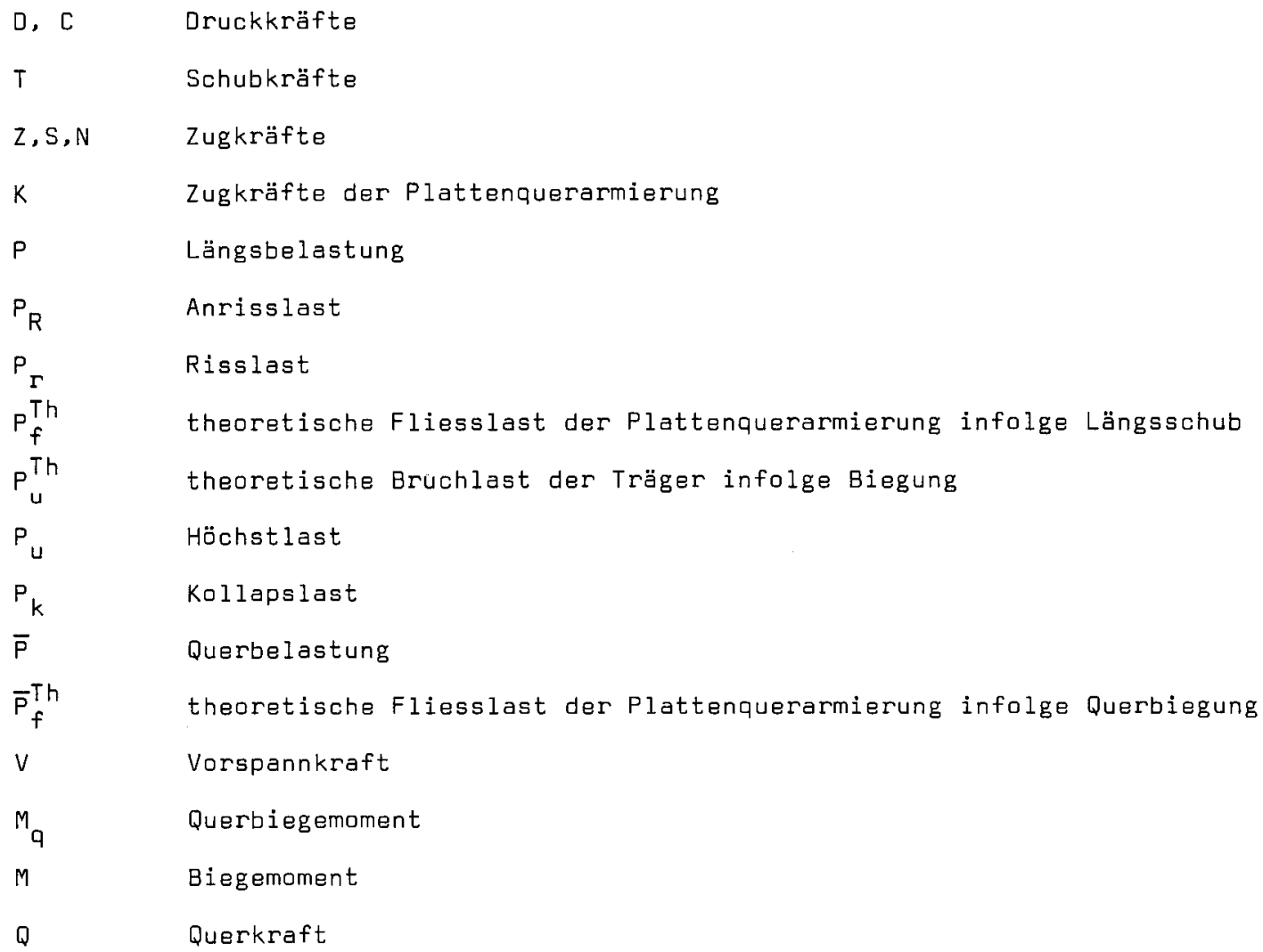




\section{Festigkeitswerte, Spannungen}

$\begin{array}{ll}\sigma_{x} & \text { Druckspannung in der Mittelebene der Platte } \\ \sigma_{z} & \text { Zugspannung } \\ \sigma_{h z} & \text { Hauptzugspannungen } \\ \sigma_{h d} & \text { Hauptdruckspannungen } \\ \beta_{W} & \text { Würfeldruckfestigkeit } \\ \beta_{P} & \text { Prismendruckfestigkeit } \\ \beta_{b z} & \text { Biegezugfestigkeit } \\ \beta_{Q z} & \text { Querzugfestigkeit } \\ \sigma_{e} & \text { Stahlspannung } \\ \sigma_{f} & \text { Fliessspannung } \\ \beta_{z} & \text { Zugfestigkeit } \\ E_{e} & \text { Elastizitätsmodul des Stahls } \\ E_{b o} & \text { Anfangs-Elastizitätsmodul des Betons } \\ \tau_{y x} & \text { Längsschubspannung im Anschluss Platte/Steg } \\ \tau_{y z} & \text { Querschubspannung im Anschluss Platte/Steg } \\ \tau_{s} & \text { Schubspannung im Steg } \\ \tau_{p} & \text { Schubspannung in der Platte } \\ \tau_{1}^{*} & \text { untere Schubspannungsgrenze } \\ 5 \tau_{1}^{*} & \text { obere Schubspannungsgrenze } \\ & \end{array}$

\section{Verformungen}

$\begin{array}{ll}\varepsilon_{b} & \text { Betonstauchungen } \\ \varepsilon_{e} & \text { Stahldehnungen } \\ \varepsilon_{e m} & \text { mittlere Stahldehnungen } \\ \varepsilon_{e \text { max }} & \text { maximale Stahldehnung im Rissequerschnitt } \\ \varepsilon_{f} & \text { Fliessdehnung } \\ \lambda_{G I} & \text { Gleichmassdehnung } \\ \varepsilon_{u} & \text { Dehnung bei Höchstlast } \\ \delta_{s} & \text { Stegdurchbiegung } \\ \delta_{u} & \text { Stegdurchbiegung bei Höchstlast } \\ \delta_{k} & \text { Stegdurchbiegung bei Kollapslast } \\ \delta_{r} & \text { Relativdurchbiegung des Plattenrandes } \\ r & \text { Rissbreite }\end{array}$




\section{Dimensionslose Parameter}

\section{$\varphi$ \\ Neigung der Hauptzugspannungen}

$\alpha$ Neigung der Betondruckdiagonalen im Steg

$\beta$ Ausbreitwinkel der Betondruckdiagonalen im Druckflansch Armierungsgehalt der Plattenquerarmierung

$\mu_{L}$ Längsarmierungsgehalt

$\mu_{B}$

Bügelarmierungsgehalt

$\mathrm{m}$ Anzahl Stegdruckdiagonalen

K Verbundkoeffizient

\section{Indizes}

I, II, III Stufen der Längs-bzw. Querbelastung

b Beton

e Stahl

Th theoretisch

Ex experimentell

A Anfangslast

Endlast

fliessen

Höchstlast

Kollapslast

d dynamisch statisch

0 oben unten längs

B Bügel




\begin{tabular}{|ll|}
\hline Sond $0 \div 4 \mathrm{~mm}$ & $829 \mathrm{~kg} / \mathrm{m}^{3}$ \\
Sond $4 \div 8 \mathrm{~mm}$ & $452 \mathrm{~kg} / \mathrm{m}^{3}$ \\
Kies $8 \div 16 \mathrm{~mm}$ & $602 \mathrm{~kg} / \mathrm{m}^{3}$ \\
Zement & $300 \mathrm{~kg} / \mathrm{m}^{3}$ \\
Wasser & $187 \mathrm{~kg} / \mathrm{m}^{3}$ \\
W/Z - Foktor & 0.62 \\
Romgewicht & $2400 \mathrm{~kg} / \mathrm{m}^{3}$ \\
Konsistenz & plastisch \\
\hline
\end{tabular}

Tabelle 1: Zusammensetzung des Frischbetons

\begin{tabular}{|c|c|c|c|c|}
\hline Balken & $\begin{array}{c}\beta_{w}^{12} \\
\mathrm{~kg} / \mathrm{cm}^{2}\end{array}$ & $\begin{array}{c}\beta_{p} \\
\mathrm{~kg} / \mathrm{cm}^{2}\end{array}$ & $\underset{\mathrm{kg} / \mathrm{cm}^{2}}{\beta_{\mathrm{bz}}}$ & $\begin{array}{c}\beta_{q z} \\
\mathrm{~kg} / \mathrm{cm}^{2}\end{array}$ \\
\hline Q1 & $\begin{array}{l}3.21 \\
(1.9)\end{array}$ & $\begin{array}{c}261 \\
(2.3)\end{array}$ & $\begin{array}{c}32 \\
(9.4)\end{array}$ & $\begin{array}{c}22 \\
(11.4)\end{array}$ \\
\hline Q 2 & $\begin{array}{r}356 \\
(3.9)\end{array}$ & $\begin{array}{l}298 \\
(6.0)\end{array}$ & $\begin{array}{c}39 \\
(7.7)\end{array}$ & $\begin{array}{c}25 \\
(10.0)\end{array}$ \\
\hline Q 3 & $\begin{array}{l}297 \\
(3.4)\end{array}$ & $\begin{array}{c}234 \\
(5.6)\end{array}$ & $\begin{array}{c}32 \\
(12.5)\end{array}$ & $\begin{array}{c}19 \\
(7.9)\end{array}$ \\
\hline Q 4 & $\begin{array}{l}364 \\
(3.3)\end{array}$ & $\begin{array}{r}285 \\
(4.9)\end{array}$ & $\begin{array}{c}41 \\
(14.6)\end{array}$ & $\begin{array}{c}21 \\
(9.5)\end{array}$ \\
\hline Q5 & $\begin{array}{l}346 \\
(4.0)\end{array}$ & $\begin{array}{r}280 \\
(5.4)\end{array}$ & $\begin{array}{c}40 \\
(12.5)\end{array}$ & $\begin{array}{c}22 \\
(20.4)\end{array}$ \\
\hline $\begin{array}{l}\text { Mittelwert } \\
\mathrm{Q} 1 \div \mathrm{Q} 5\end{array}$ & $\begin{array}{c}337 \\
(8.0)\end{array}$ & $\begin{array}{r}271 \\
(9.6)\end{array}$ & $\begin{array}{c}37 \\
(16.2)\end{array}$ & $\begin{array}{c}22 \\
(13.6)\end{array}$ \\
\hline
\end{tabular}

Tabelle 2: Konventionelle Festigkeits werte des Betons nach 28 Tagen

\begin{tabular}{|c|c|c|c|c|c|c|c|c|c|c|}
\hline Prismo & $\begin{array}{c}\dot{\varepsilon}=\Delta \mathcal{E} / \Delta t \\
10^{-3} / \mathrm{min}\end{array}$ & $\begin{array}{c}\mathrm{t}_{w} \\
\mathrm{~min}\end{array}$ & $\begin{array}{c}\beta_{p d} \\
\mathrm{~kg} / \mathrm{cm}^{2}\end{array}$ & $\begin{array}{c}\beta_{p s}\left(2^{\prime}\right) \\
\mathrm{kg} / \mathrm{cm}^{2}\end{array}$ & $\begin{array}{c}\beta_{p s}\left(30^{\prime}\right) \\
\mathrm{kg} / \mathrm{cm}^{2}\end{array}$ & $\begin{array}{c}\beta_{\mathrm{ps}}\left(2^{\prime}\right) \\
\beta_{p d}\end{array}$ & $\frac{\beta_{p s}\left(30^{\prime}\right)}{\beta_{p d}}$ & $\begin{array}{c}\varepsilon_{u} \\
\%\end{array}$ & $\begin{array}{c}\varepsilon_{\max } \\
\%\end{array}$ & $\begin{array}{c}E_{b}(5 \div 100) \\
t / \mathrm{cm}^{2}\end{array}$ \\
\hline 01 & 0.15 & 2 & 240 & 215 & & 0.90 & & 1.65 & 3.75 & 320 \\
02 & 0.15 & 2 & 282 & 253 & & 0.90 & & 1.82 & 2.25 & 302 \\
03 & 0.15 & 2 & 209 & 186 & & 0.89 & & 1.54 & 3.36 & 291 \\
Q4 & 0.15 & 2 & 248 & 223 & & 0.90 & & 1.70 & 2.70 & 317 \\
05 & 0.15 & 2 & 242 & 219 & & 0.90 & & 1.72 & 2.70 & 259 \\
\hline
\end{tabular}

* Wert ous früheren Versuchen übernommen

Tabelle 3: Spannungs - Dehnungs - Verhalten der Betonprismen

\begin{tabular}{|c|c|c|c|c|c|c|c|c|c|c|c|}
\hline $\begin{array}{c}\text { Nenn- } \\
\varnothing \\
\mathrm{mm}\end{array}$ & $\begin{array}{l}\text { Anzahl } \\
\text { Stábe }\end{array}$ & $\begin{array}{l}F_{\text {eff }} \\
\mathrm{cm}^{2}\end{array}$ & $\begin{array}{c}\varepsilon \\
\% / \min \end{array}$ & $\begin{array}{l}\sigma_{\mathrm{fd}}(\dot{\xi}) \\
t / \mathrm{cm}^{2}\end{array}$ & $\begin{array}{c}\sigma_{\mathrm{fs}}(\dot{\dot{\varepsilon}}=0) \\
t / \mathrm{cm}^{2}\end{array}$ & $\begin{array}{l}\beta_{z \alpha}(\dot{\varepsilon}) \\
\dagger / \mathrm{cm}^{2}\end{array}$ & $\begin{array}{c}\beta_{z s}(\dot{\varepsilon}=0) \\
t / \mathrm{cm}^{2}\end{array}$ & $\begin{array}{c}\varepsilon_{\text {ev }} \\
\%\end{array}$ & $\begin{array}{c}\lambda \\
\text { gemessen } \\
\%\end{array}$ & $\begin{array}{l}\psi \\
\%\end{array}$ & $\begin{array}{l}\text { Stahl- } \\
\text { qualitdt }\end{array}$ \\
\hline 12 & 3 & $\begin{array}{l}0.281 \\
(2.0)\end{array}$ & 1.25 & $\begin{array}{c}4.92 \\
(5.4)\end{array}$ & $\begin{array}{l}4.75 \\
(5.7)\end{array}$ & $\begin{array}{l}5.42 \\
(6.4)\end{array}$ & $\begin{array}{c}5.18 \\
(7.0)\end{array}$ & 2.1 & 6.6 & 62 & $\begin{array}{c}\text { Tor } \\
\text { (wormebe } \\
\text { handelt) }\end{array}$ \\
\hline 12 & 3 & 1.08 & 1.25 & 5.19 & 4.95 & 7.0 & 6.66 & 1.8 & 16.2 & 56 & Box- \\
\hline 30 & 3 & 6.96 & 1.25 & 5.79 & 5.54 & $7.92^{*}$ & $\sim 7.50^{* *}$ & 1.3 & 13.8 & 44 & ultra \\
\hline
\end{tabular}

Klommerwerfe $=$ Varloflonskoeffizienten in \%

* Mittel ous zwei konventionellen Zugversuchen ** geschidtzt

Tabelle 4 : Eigenschaften des verwendeten Stahles (gemäss dehnungsgesteuerten Zugversuchen) 


\begin{tabular}{|c|c|c|}
\hline \multirow{5}{*}{ m } & $\underset{10}{\equiv}$ & 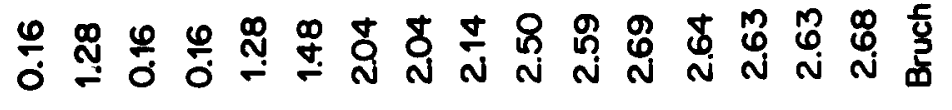 \\
\hline & $\mid \begin{array}{ll}\Xi \\
10^{\alpha}\end{array}$ & 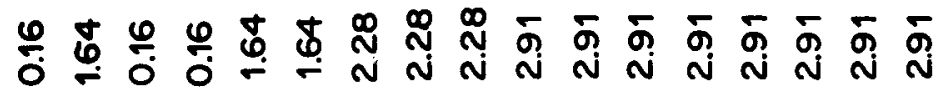 \\
\hline & $\equiv$ & 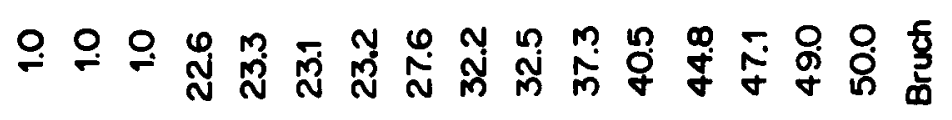 \\
\hline & $\begin{array}{l}\equiv \\
a^{\alpha}\end{array}$ & 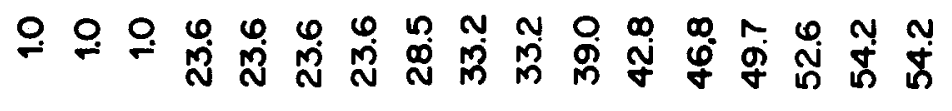 \\
\hline & 3 & $0-N m$ \\
\hline
\end{tabular}

\begin{tabular}{|c|c|c|}
\hline \multirow{3}{*}{ N } & $\equiv$ & 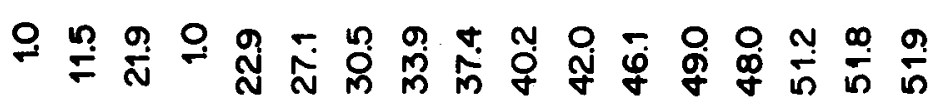 \\
\hline & $\begin{array}{l}\equiv \\
a^{4}\end{array}$ & 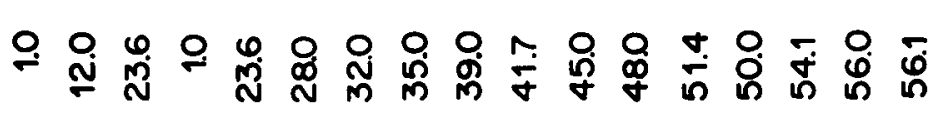 \\
\hline & 3 & 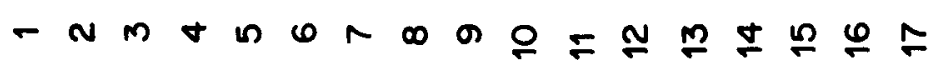 \\
\hline
\end{tabular}




\begin{tabular}{|c|c|c|}
\hline \multirow{5}{*}{ n } & $\sum_{10}$ & 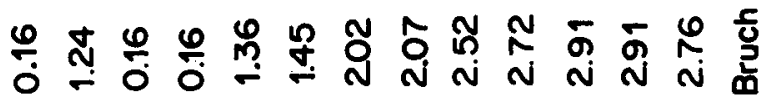 \\
\hline & $\Xi_{10^{\alpha}}$ & 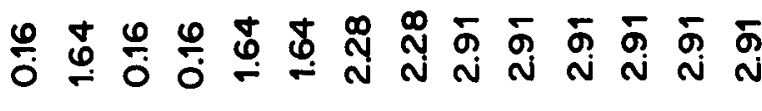 \\
\hline & $\sum_{0}^{\Xi}$ & 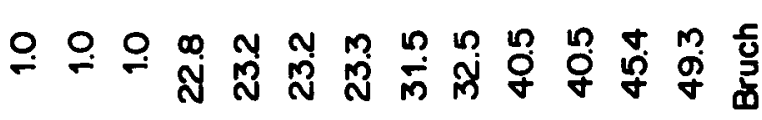 \\
\hline & $\equiv_{Q^{\mathbb{Q}}}$ & 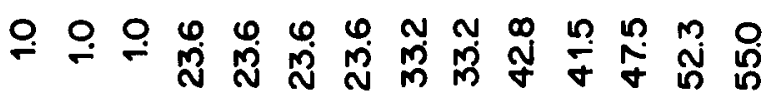 \\
\hline & g & $-N m+n \in \sim \infty \sigma O=\cong \mathbb{I}$ \\
\hline
\end{tabular}

\begin{tabular}{|c|c|c|}
\hline \multirow{5}{*}{$\begin{array}{l}0 \\
0\end{array}$} & $\sum_{10}^{\Xi}$ & 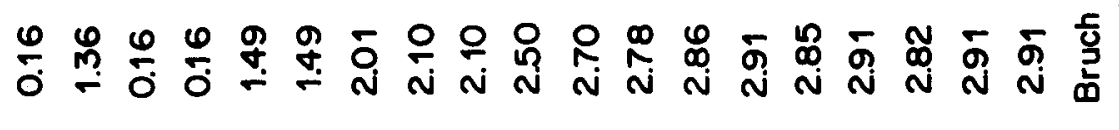 \\
\hline & $\begin{array}{l}\equiv \\
10^{4}\end{array}$ & 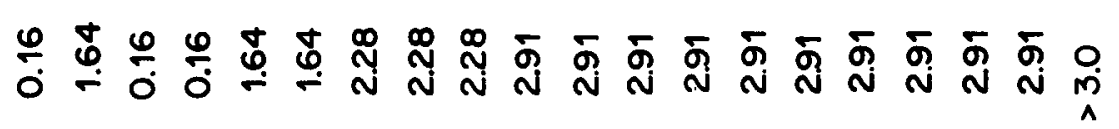 \\
\hline & $\begin{array}{l}\equiv \\
\omega \\
a\end{array}$ & 운 우 N \\
\hline & $\equiv$ & 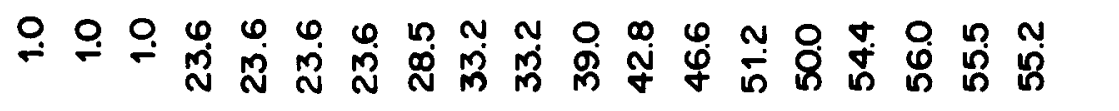 \\
\hline & s & $\stackrel{\infty}{\infty}$ \& \\
\hline
\end{tabular}




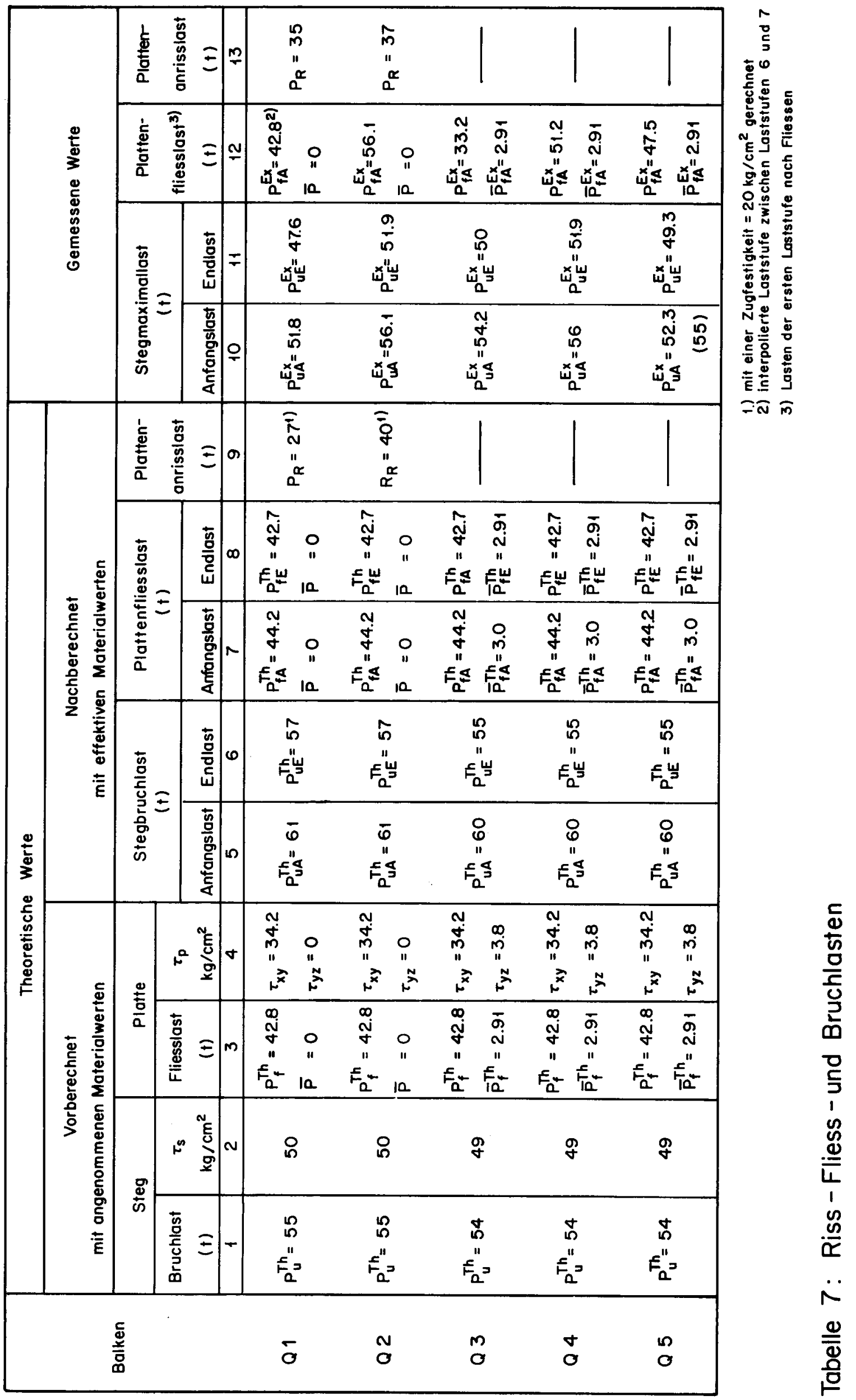




\begin{tabular}{|c|c|c|}
\hline \multirow{2}{*}{$\begin{array}{l}n \\
0\end{array}$} & : & \\
\hline & 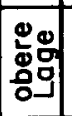 & 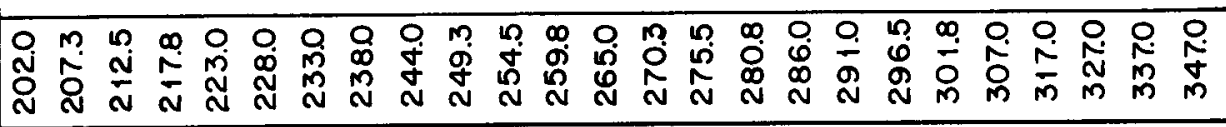 \\
\hline \multirow{2}{*}{$\stackrel{a}{a}$} & 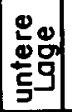 & \\
\hline & 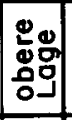 & 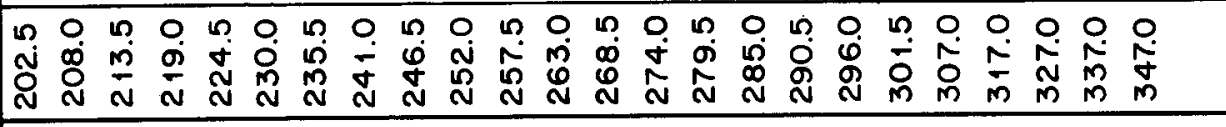 \\
\hline \multirow{2}{*}{ m } & 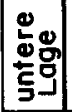 & \\
\hline & 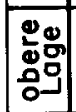 & 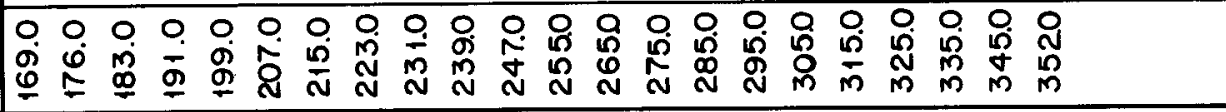 \\
\hline \multirow{2}{*}{$\begin{array}{l}n \\
0\end{array}$} & \begin{tabular}{ll|} 
\\
\\
\end{tabular} & 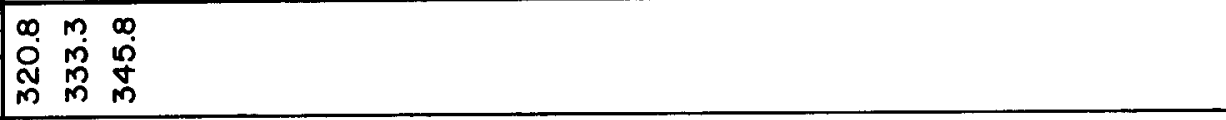 \\
\hline & 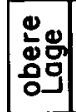 & 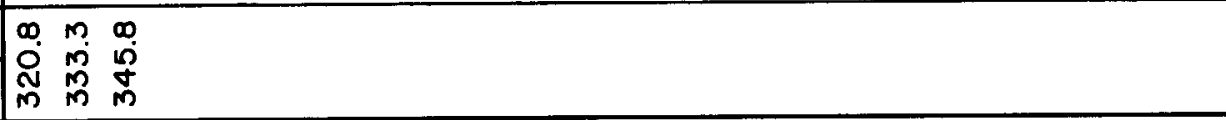 \\
\hline & $\frac{1}{2}$ & 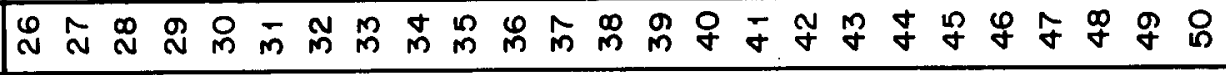 \\
\hline
\end{tabular}

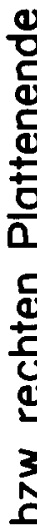

\begin{tabular}{|c|c|c|}
\hline & 产高: & 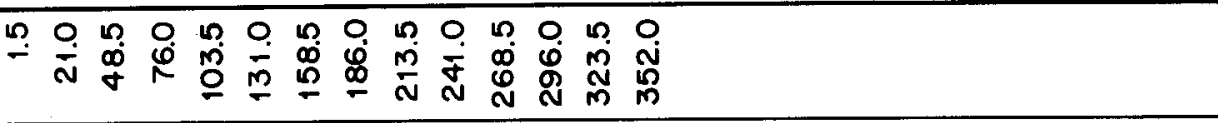 \\
\hline & 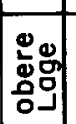 & மூ \\
\hline$\sigma$ & 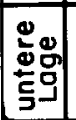 & 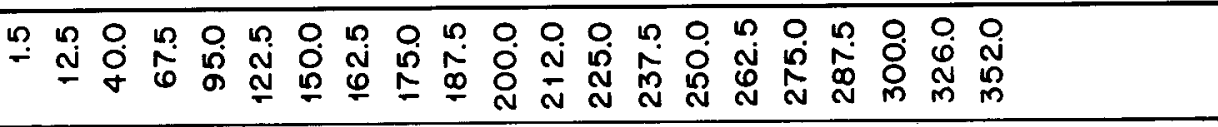 \\
\hline 0 & \begin{tabular}{|l|l|} 
& 0 \\
\\
\end{tabular} & 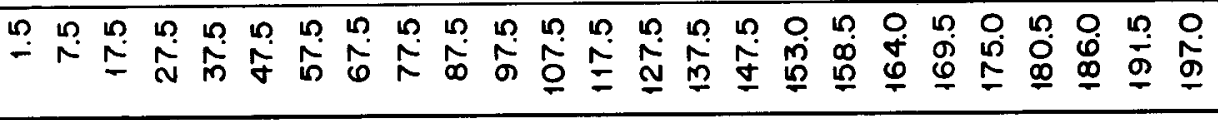 \\
\hline$m$ & 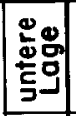 & 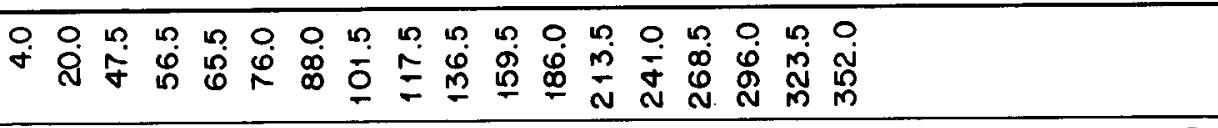 \\
\hline 0 & | - n & 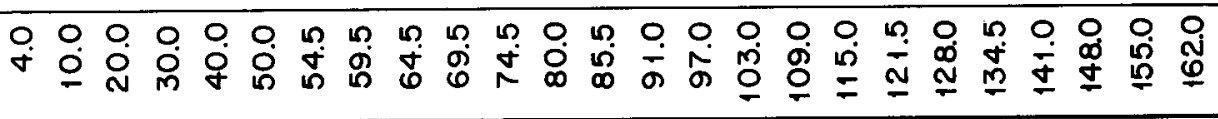 \\
\hline N & 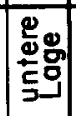 & 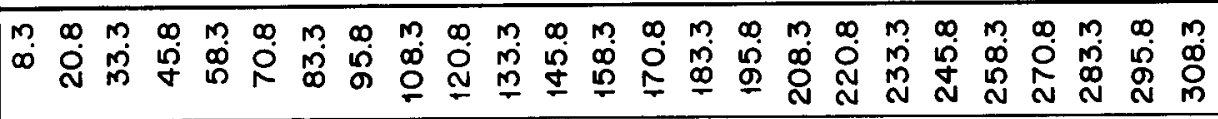 \\
\hline 0 & | & 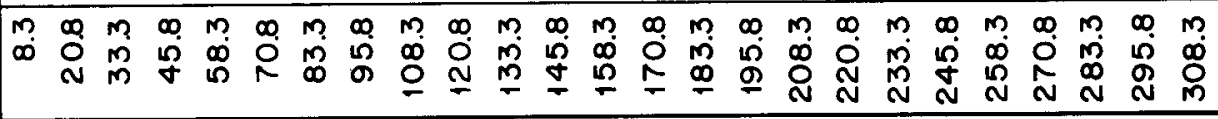 \\
\hline$\sigma$ & 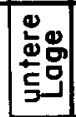 & 品 \\
\hline 0 & $\mid$\begin{tabular}{ll}
0 \\
2 \\
\hdashline
\end{tabular} & O \\
\hline & 5 & 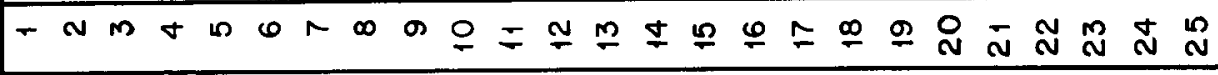 \\
\hline
\end{tabular}



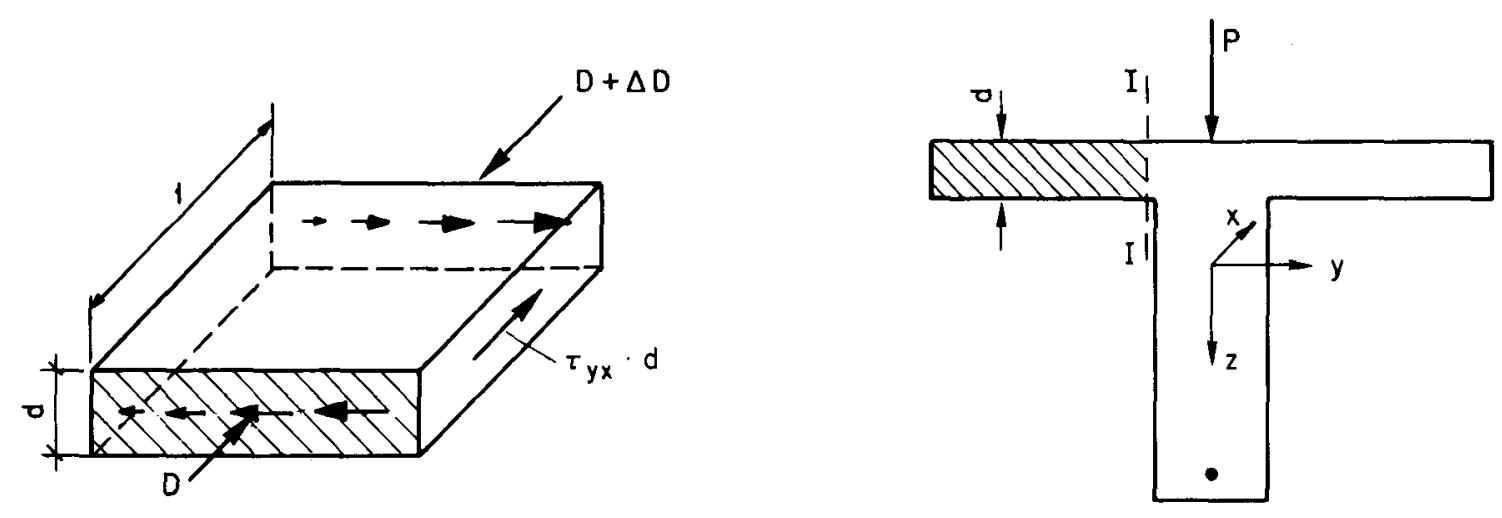

a-Längsschub allein
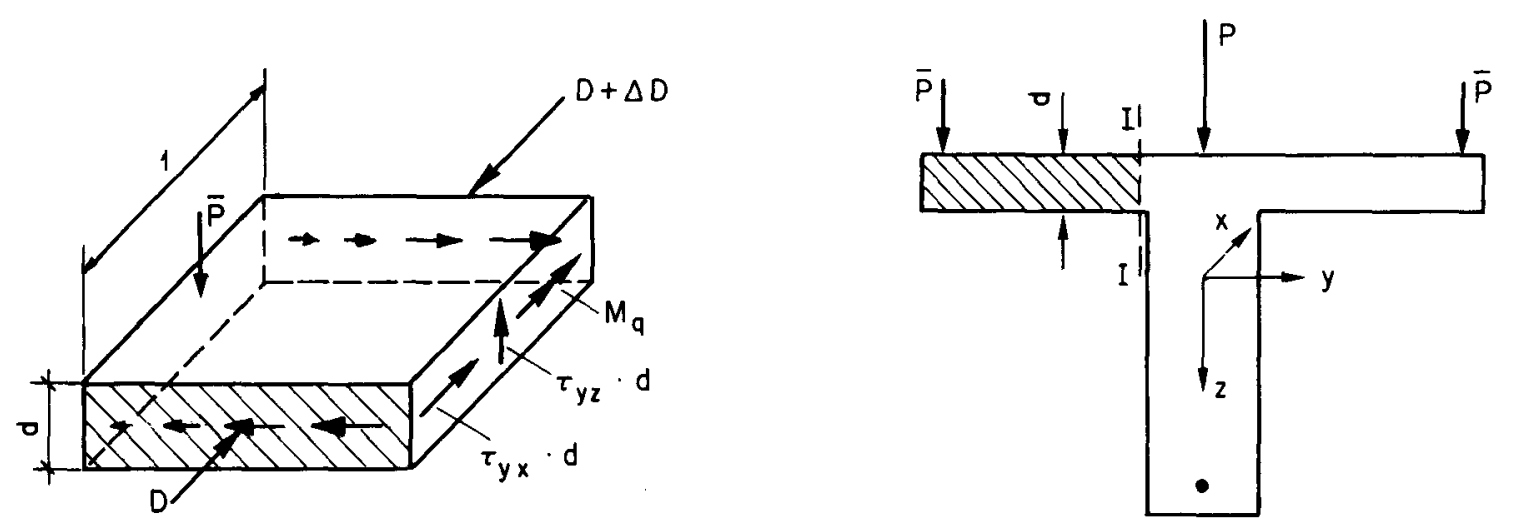

$\underline{b-L a ̈ n g s s c h u b}+$ Querbiegung
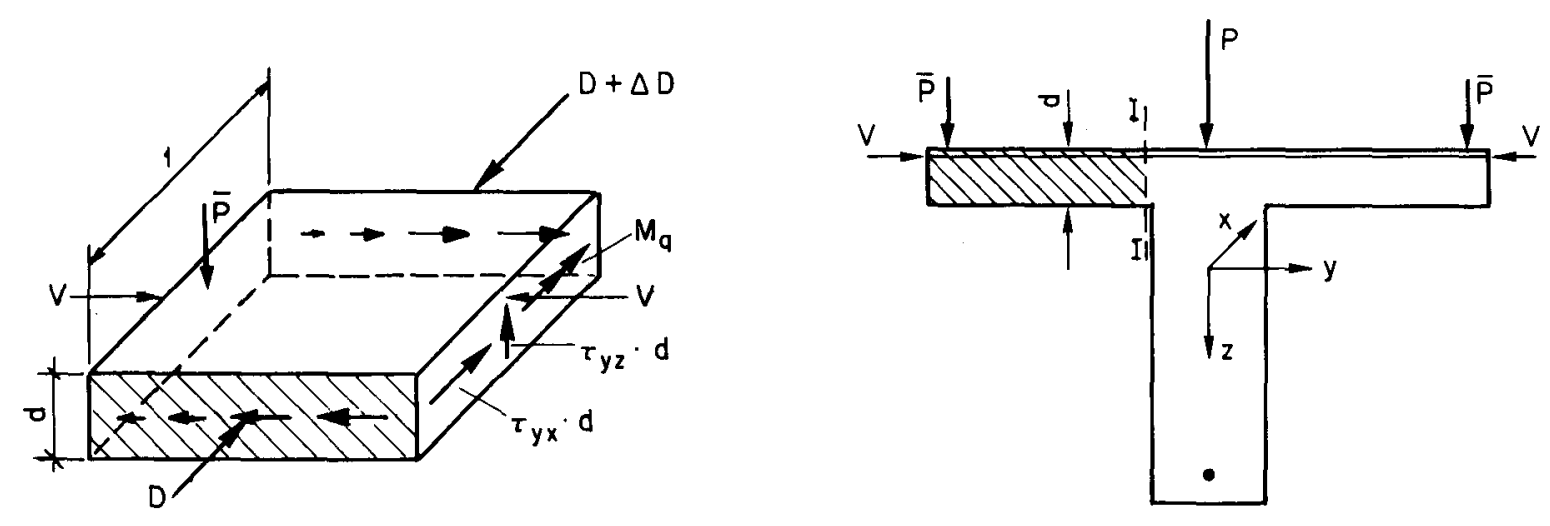

$\underline{c-L a ̈ n g s s c h u b}+\underline{\text { Querbiegung + Quervorspannung }}$

Bild 1: Problemstellung 

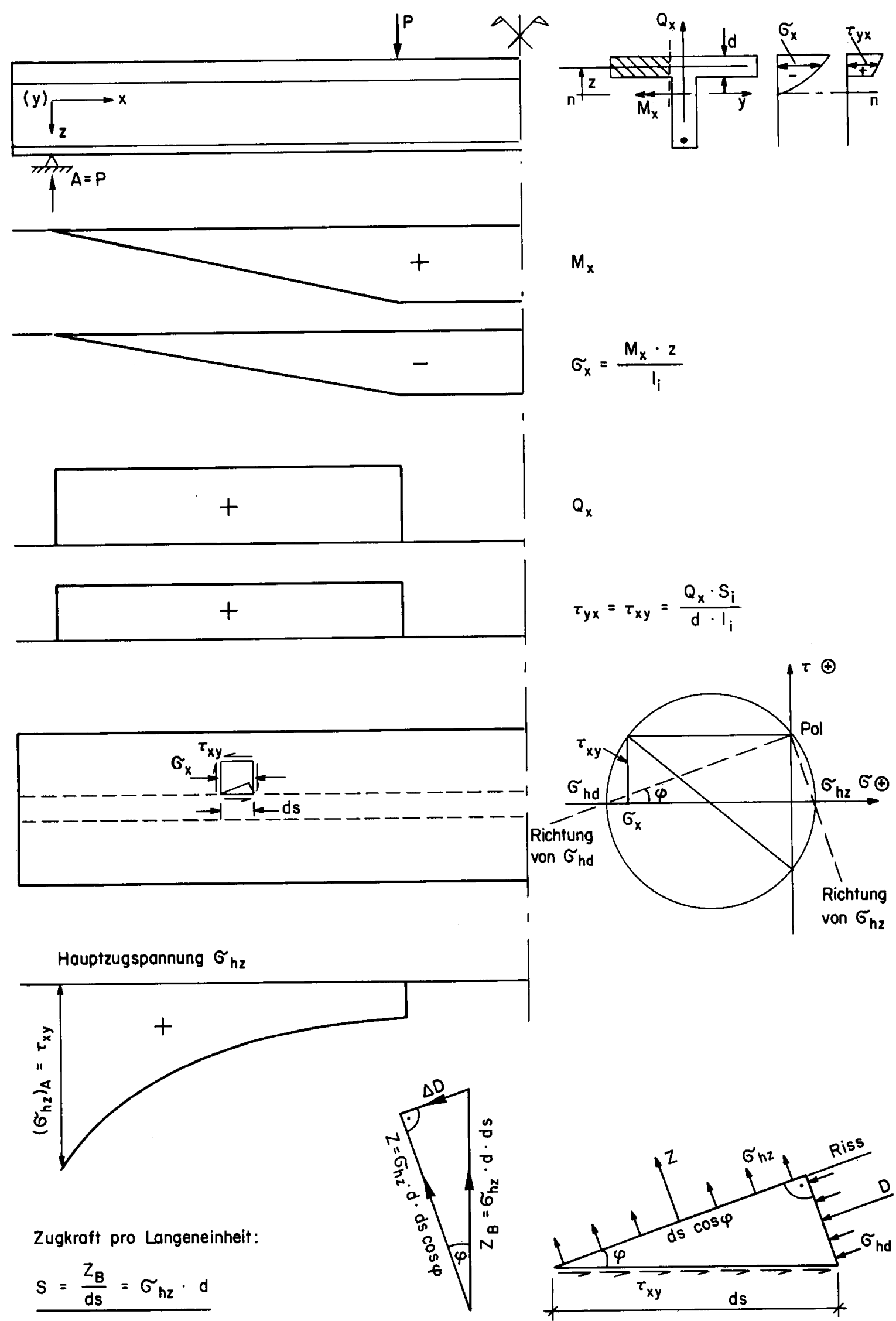

Bild 2: Klassisches Modell: Einfache Balkentheorie, (Hauptzugspannungen). 

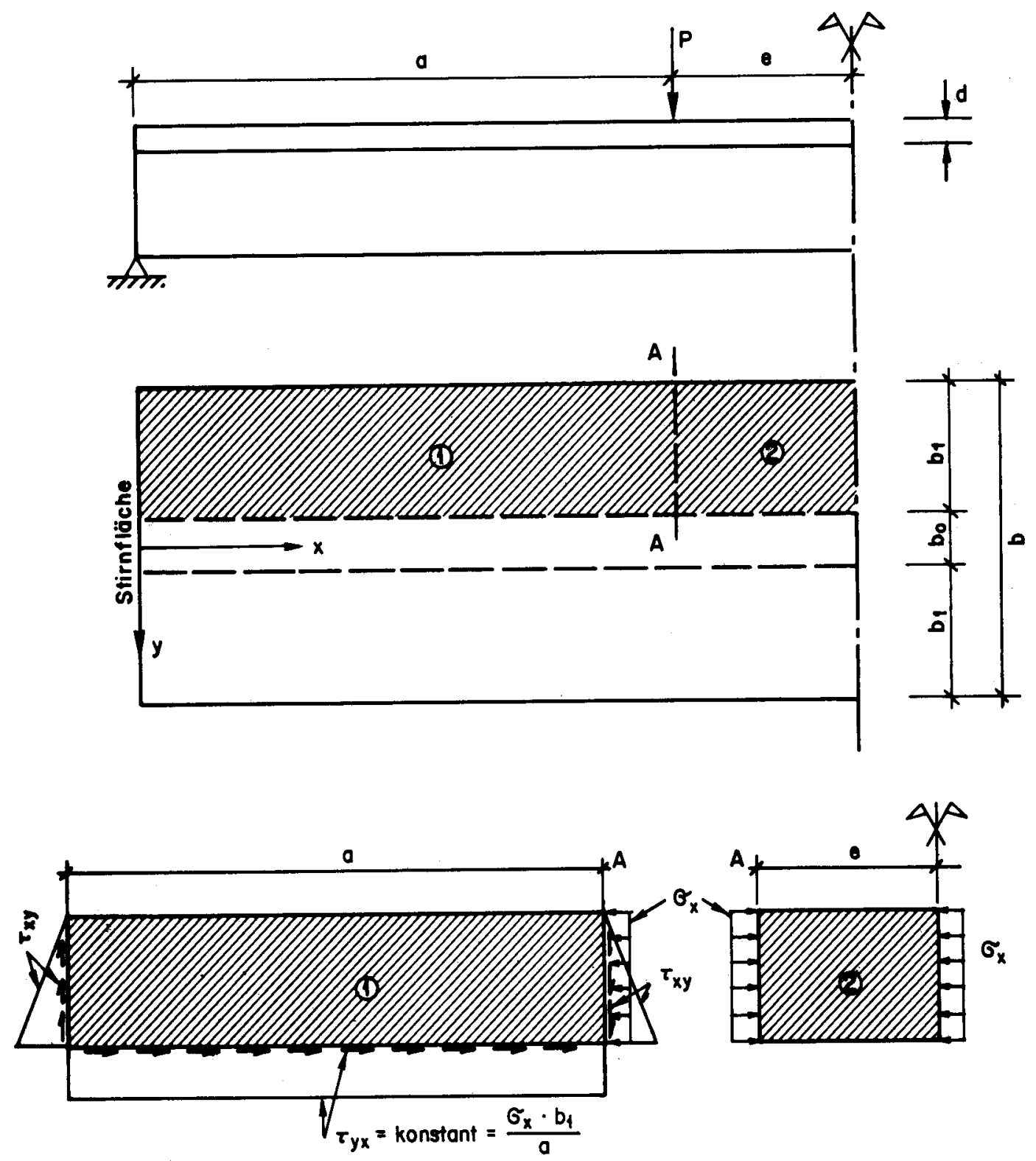

a) Spannungen an den zwei Plattenteilen (1) und (2)

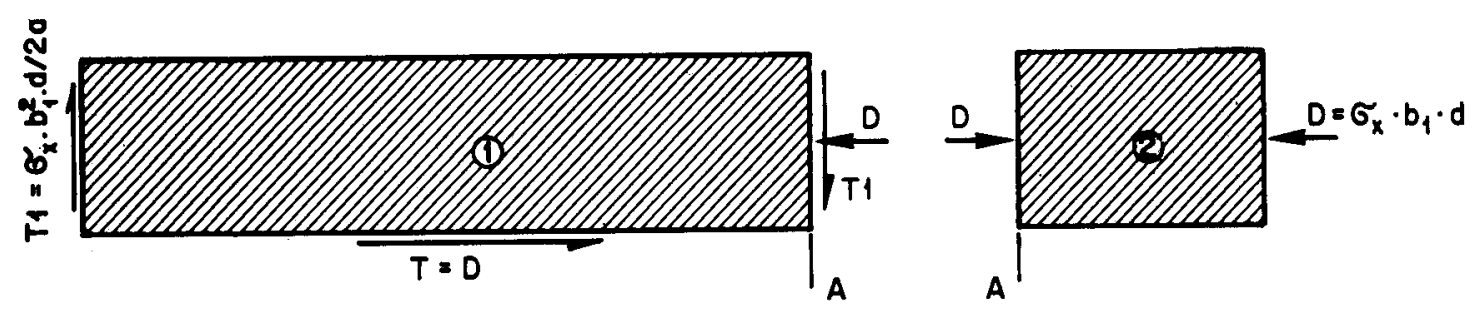

b) Krafte an den zwei Plattenteilen (1) und (2) 

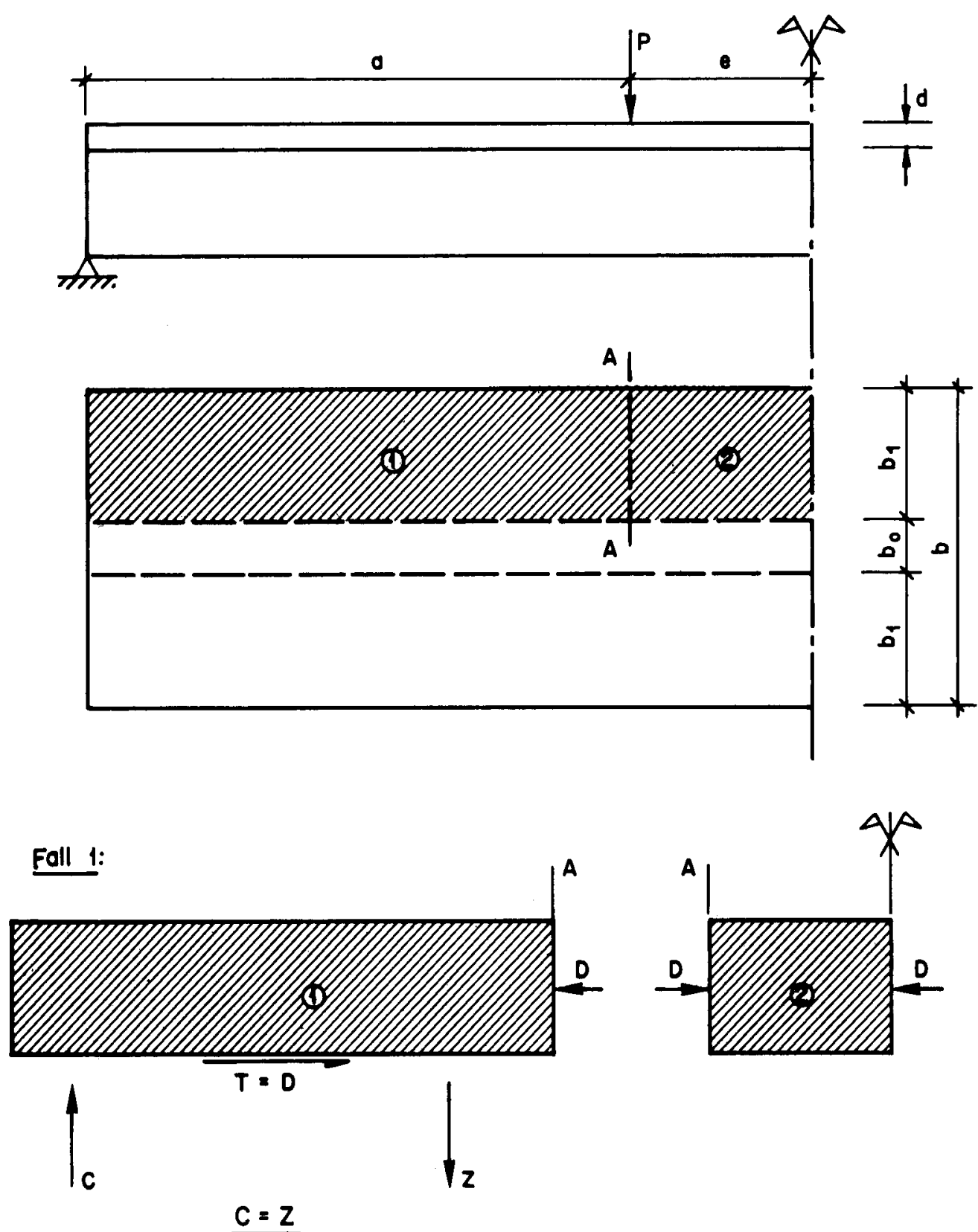

Foll 2:

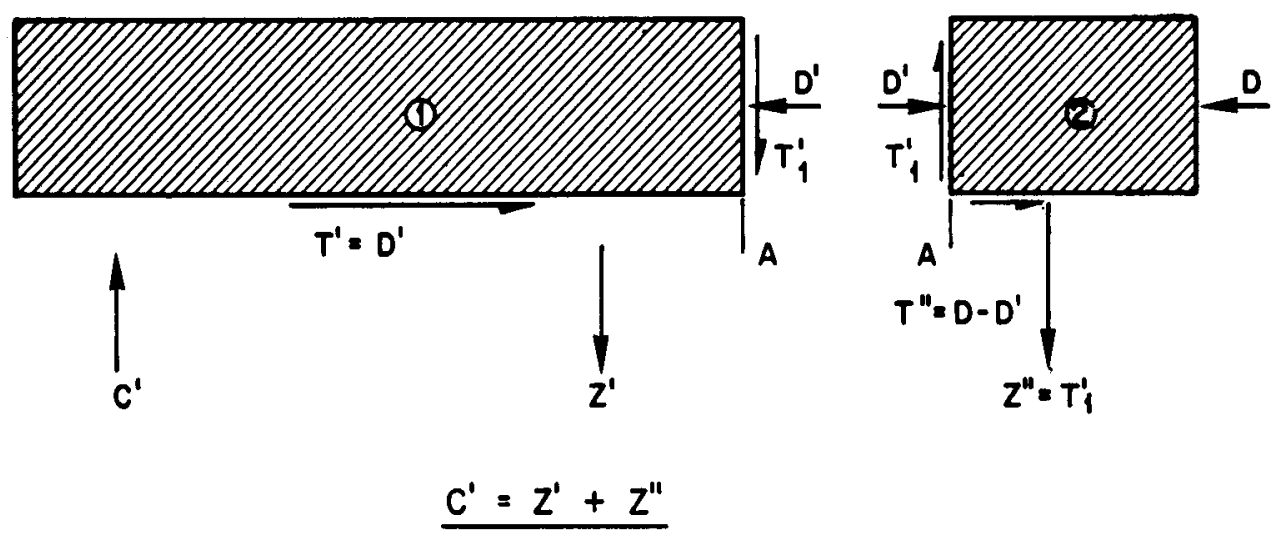

Bild 3-b: Mögliche Gleichgewichtszustände in der Platte 


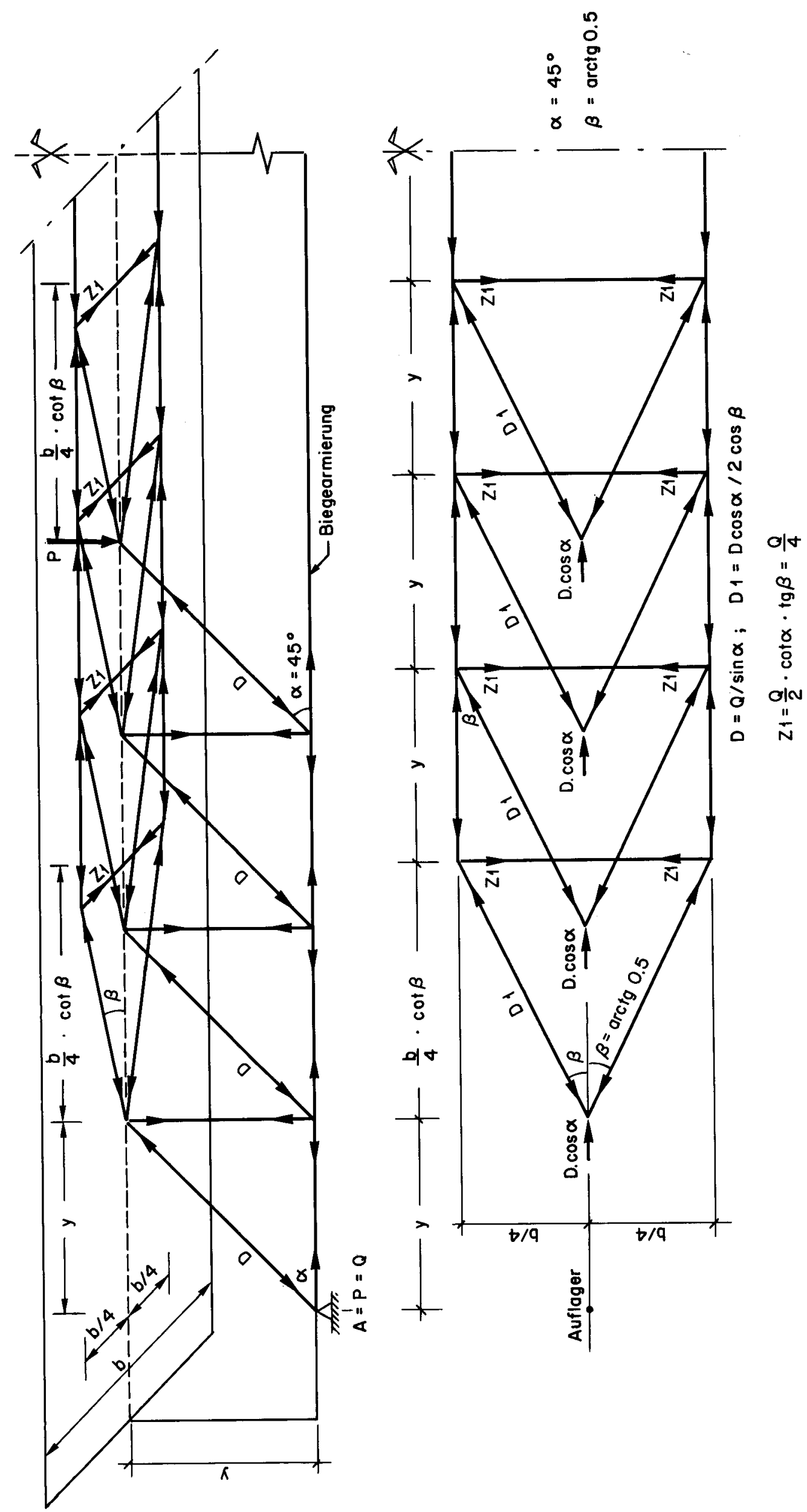

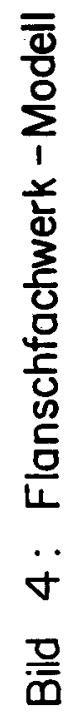




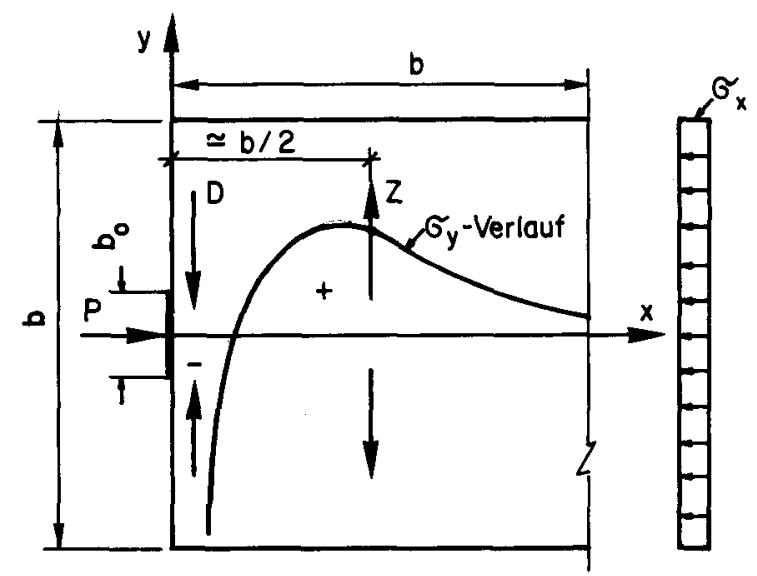

a- elastische Verteilung

von $\sigma_{y}($ bei $y=0)$ für $b_{0} / b=0.2$

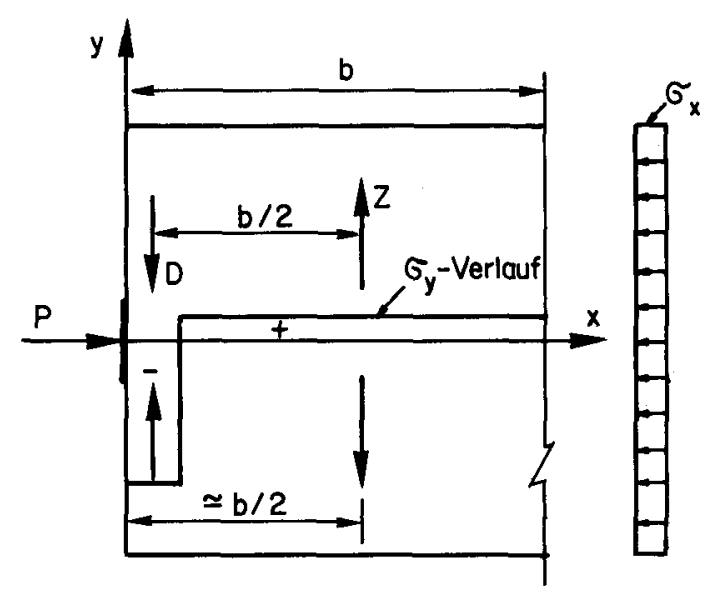

b-plastische Verteilung

von $\sigma_{y}$

nach $a$ und $b$ tritt die Zugkraft $z$ bei $x \simeq \frac{b}{2}$ auf.
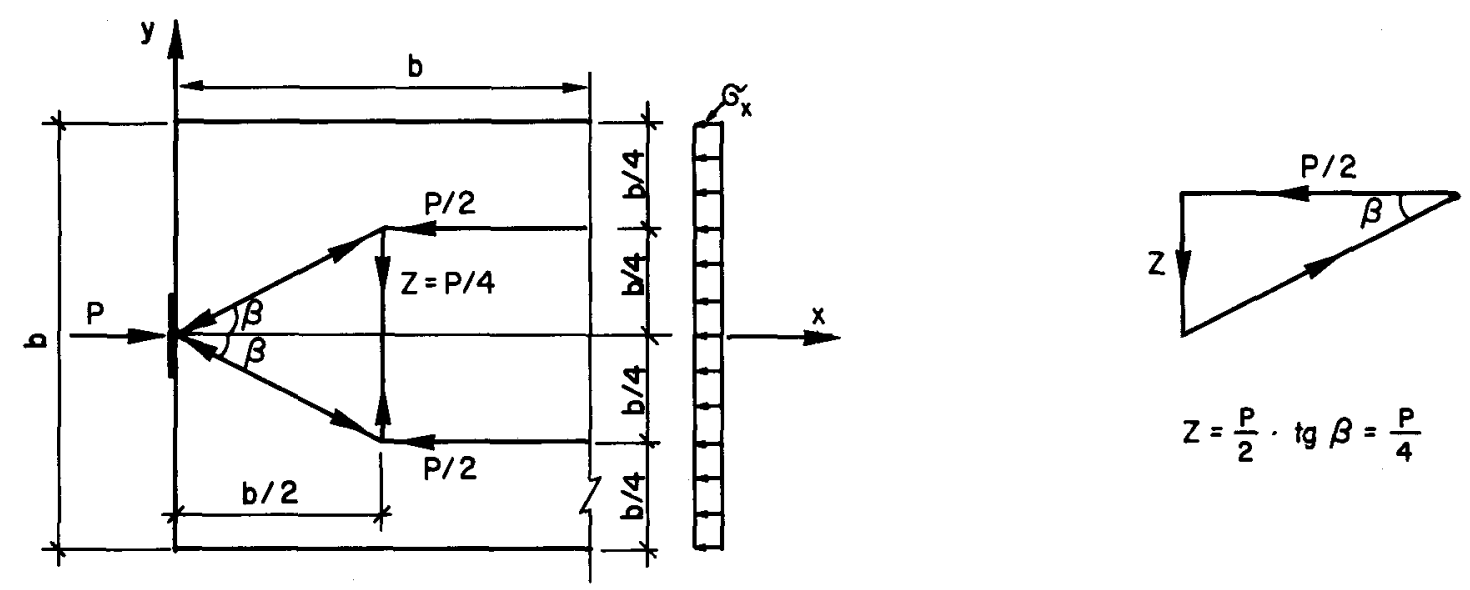

$$
Z=\frac{P}{2} \cdot \operatorname{tg} \beta=\frac{P}{4}
$$

c - Fochwerkmodell

$\operatorname{tg} \beta=\frac{\mathrm{b} / 4}{\mathrm{~b} / 2}=\underline{0.5}$

Bild 5: Abschätzung des Ausbreitungswinkels $(\beta)$ einer Kraft $P$ in Halbstreifen. 


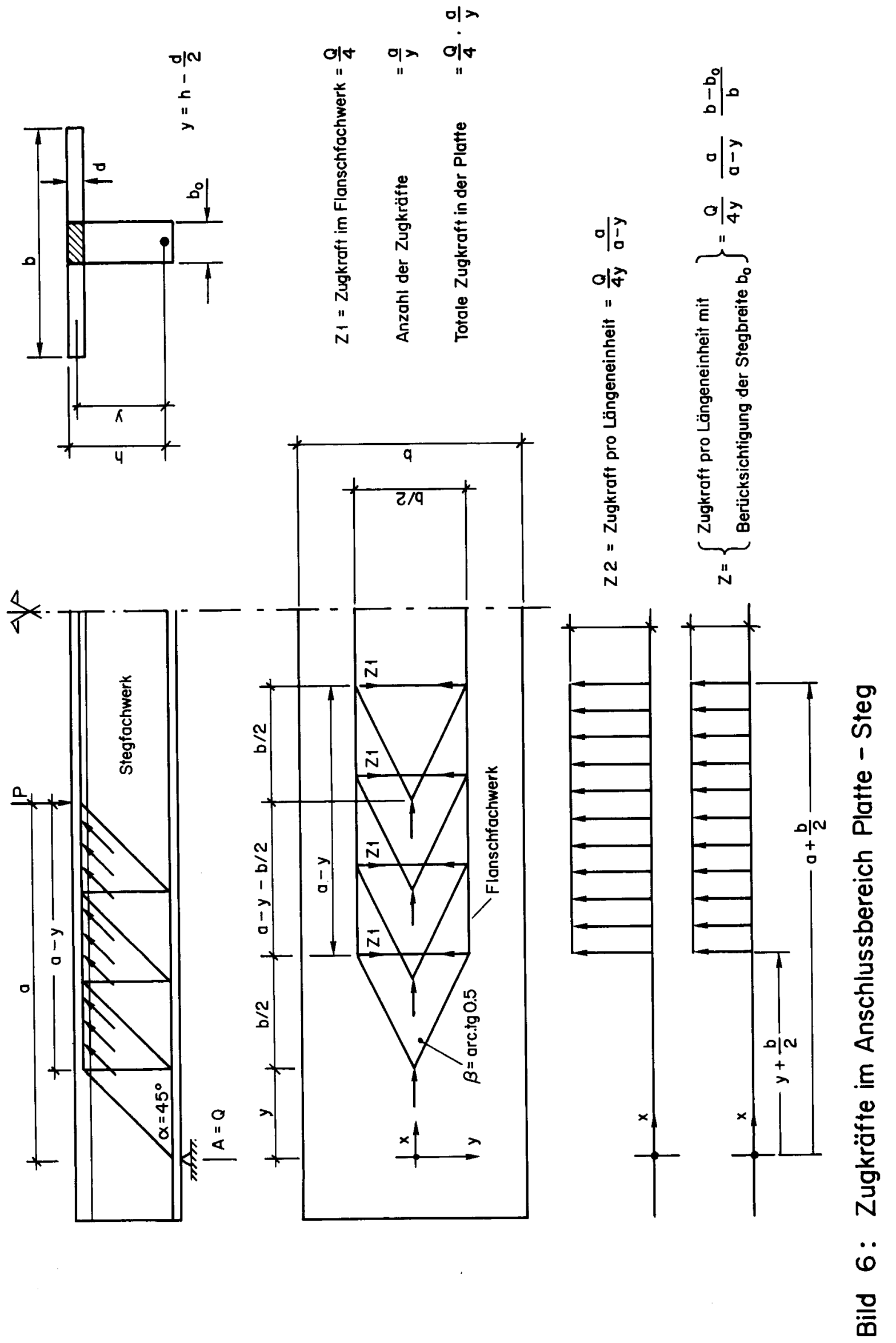



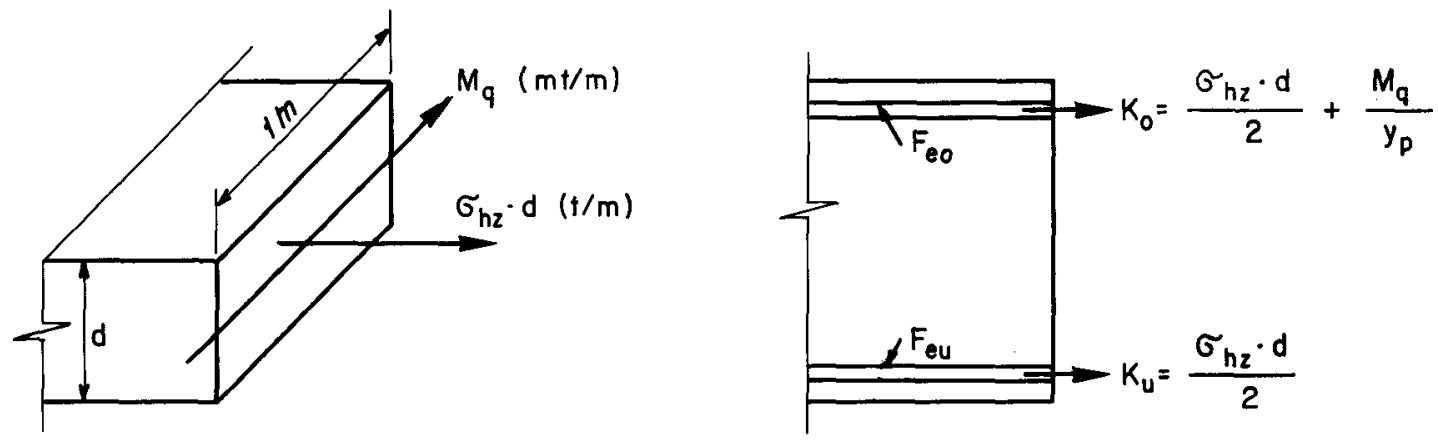

$$
a-\begin{aligned}
& \text { Superposition der Armierungen "klassisch" und Biegung } \\
& F_{e 0}=\frac{1}{2} F_{e}\left(G_{h z}\right)+F_{e}\left(M_{q}\right) ; \quad F_{e u}=\frac{1}{2} F_{e}\left(G_{h z}\right)
\end{aligned}
$$
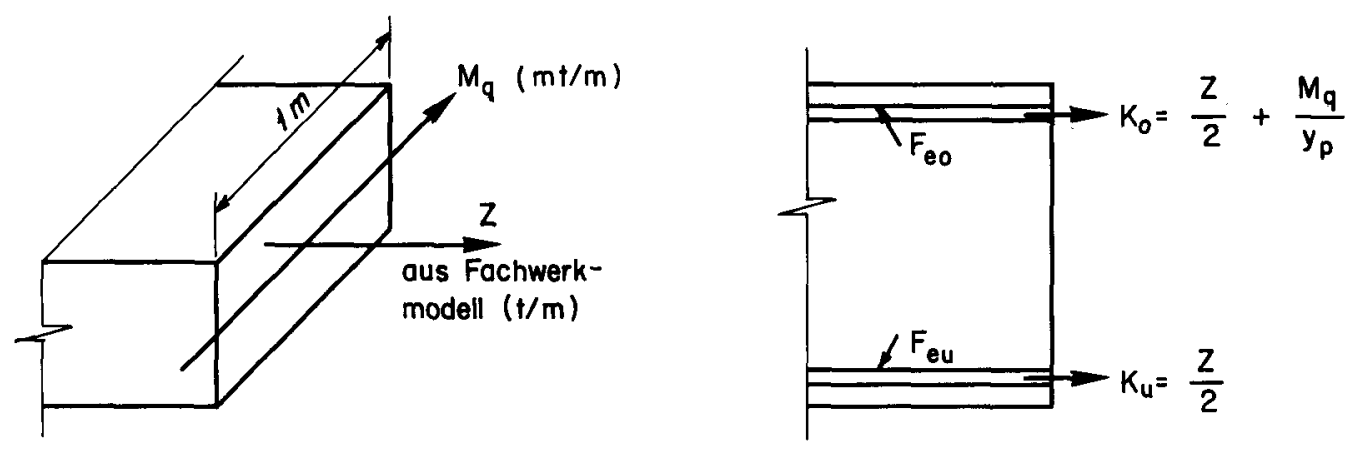

$$
\begin{aligned}
& \text { Superposition der Armierungen "Fachwerk" und Biegung } \\
& F_{e o}=\frac{1}{2} F_{e}(Z)+F_{e}\left(M_{q}\right) ; \quad F_{e u}=\frac{1}{2} F_{e}(Z)
\end{aligned}
$$
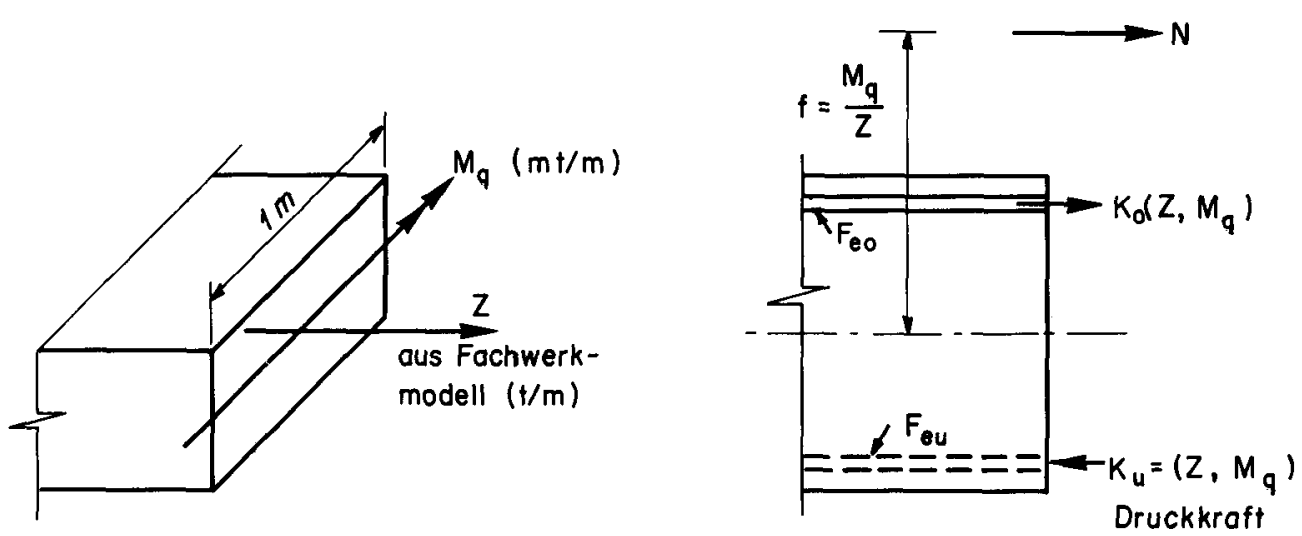

$$
\begin{aligned}
& \text { Biegung mit Axial-Zugkraft "Fachwerk" } \\
& F_{e_{0}}=f\left(Z, M_{q}\right) ; \quad F_{e u}=\text { nicht nötig }
\end{aligned}
$$




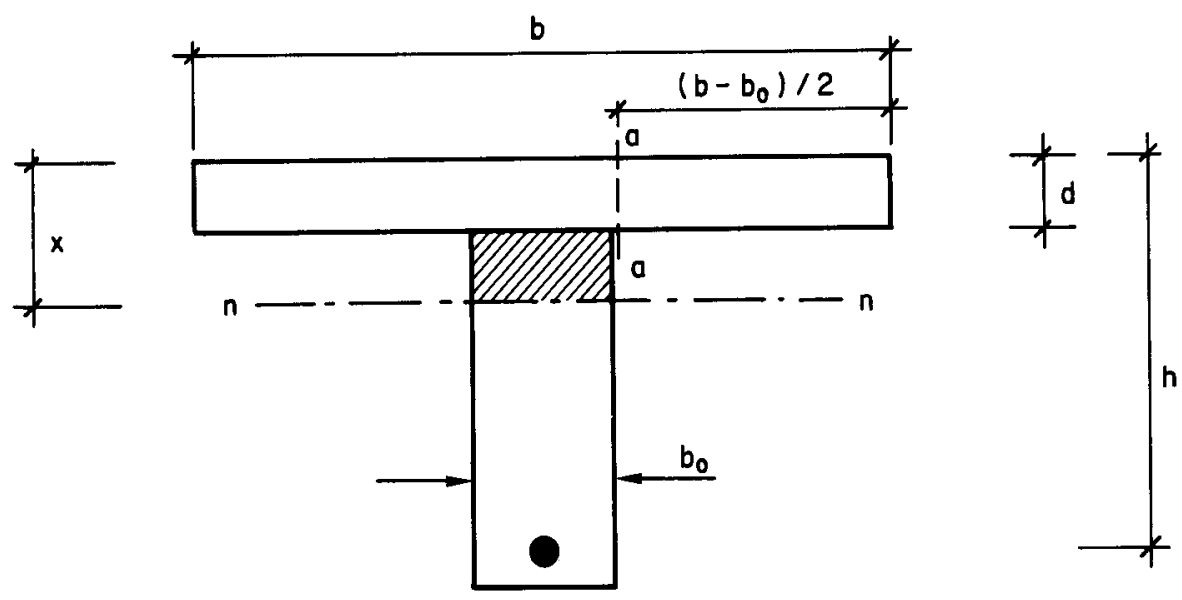

Bild 8: Querschnitt eines Plattenbalkens

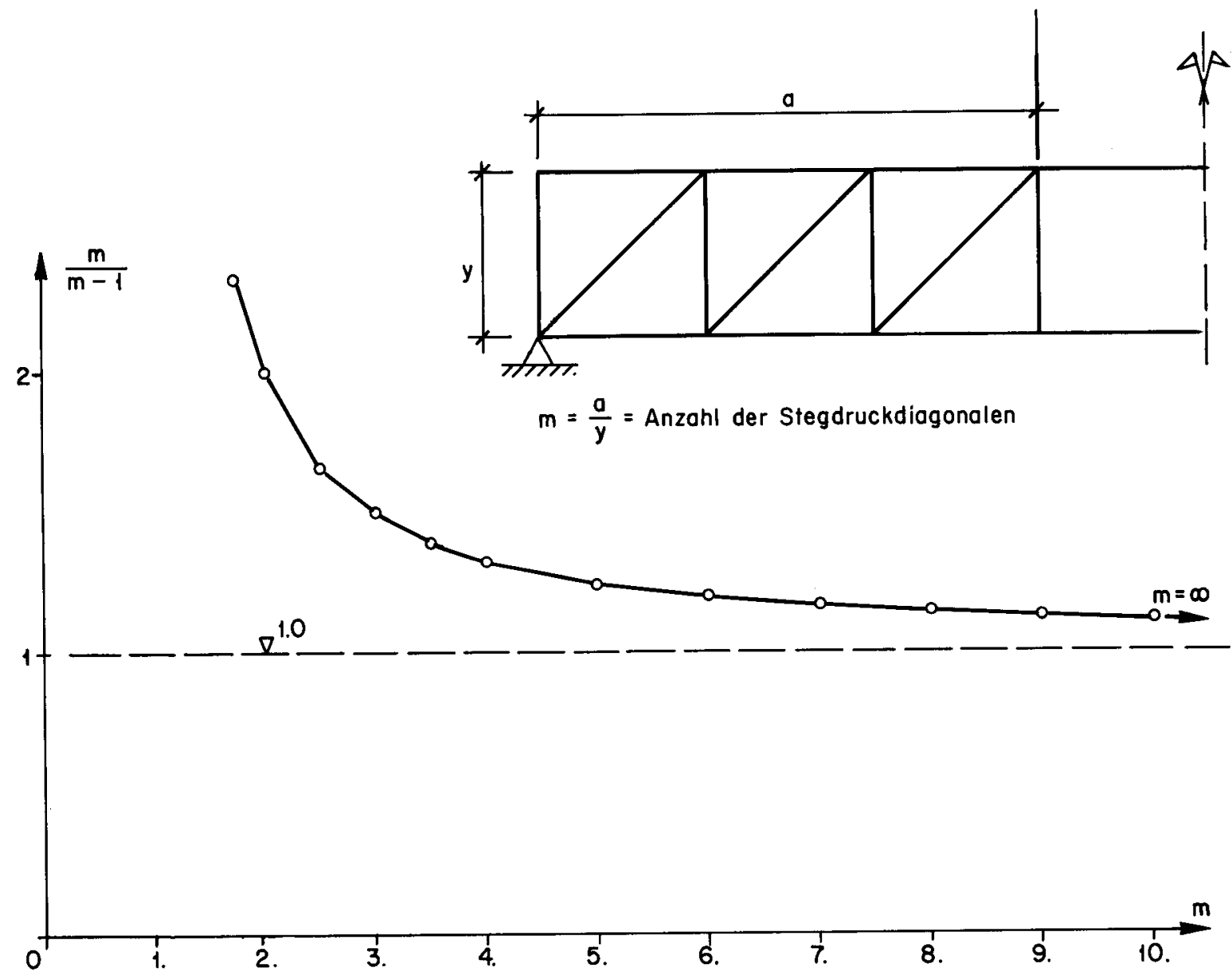

Bild 9 : Einfluss der Stegdruckdiagonalen - Anzahl auf $\sigma_{z}$ 


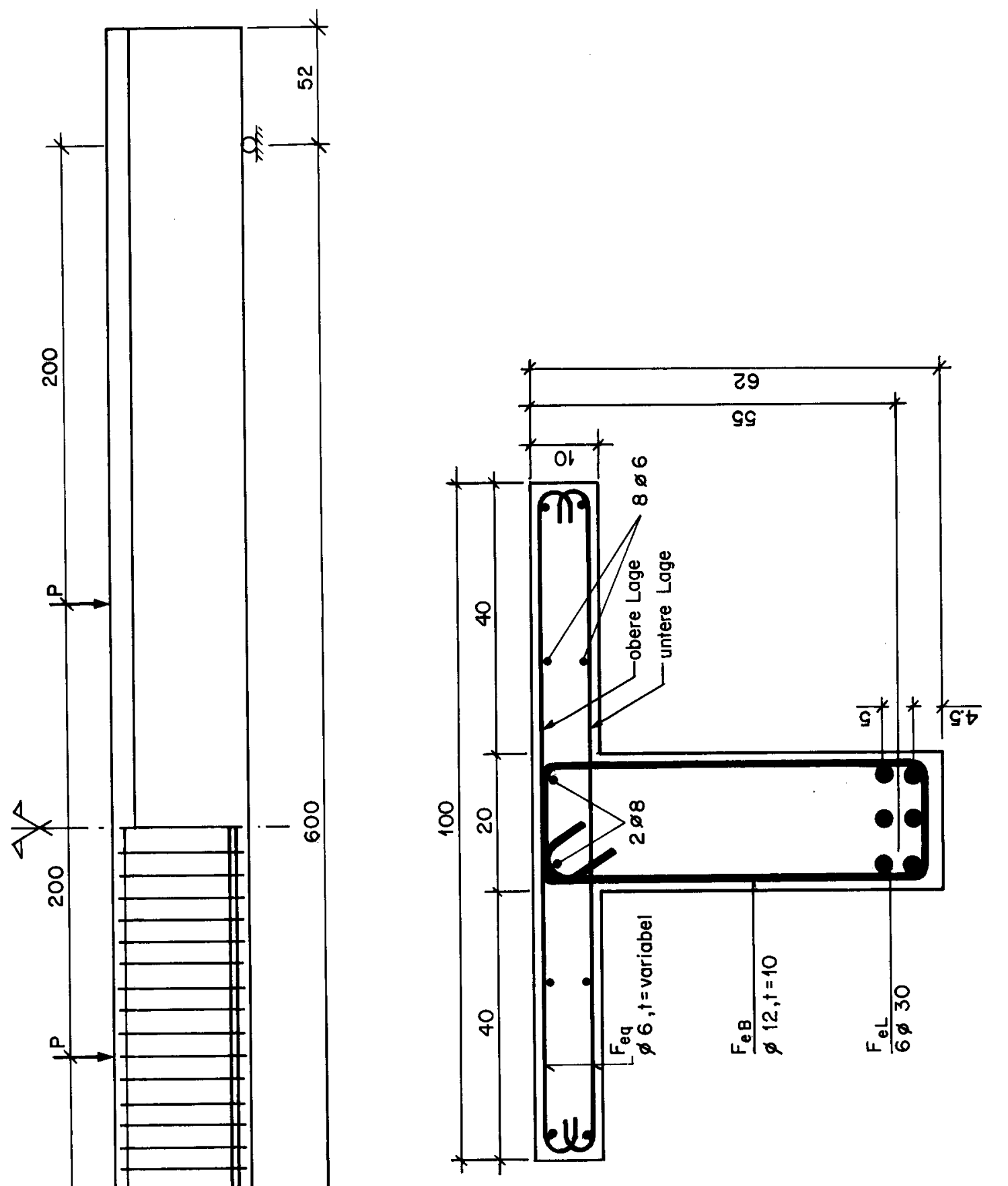

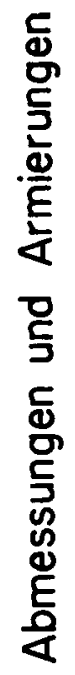

우

产 


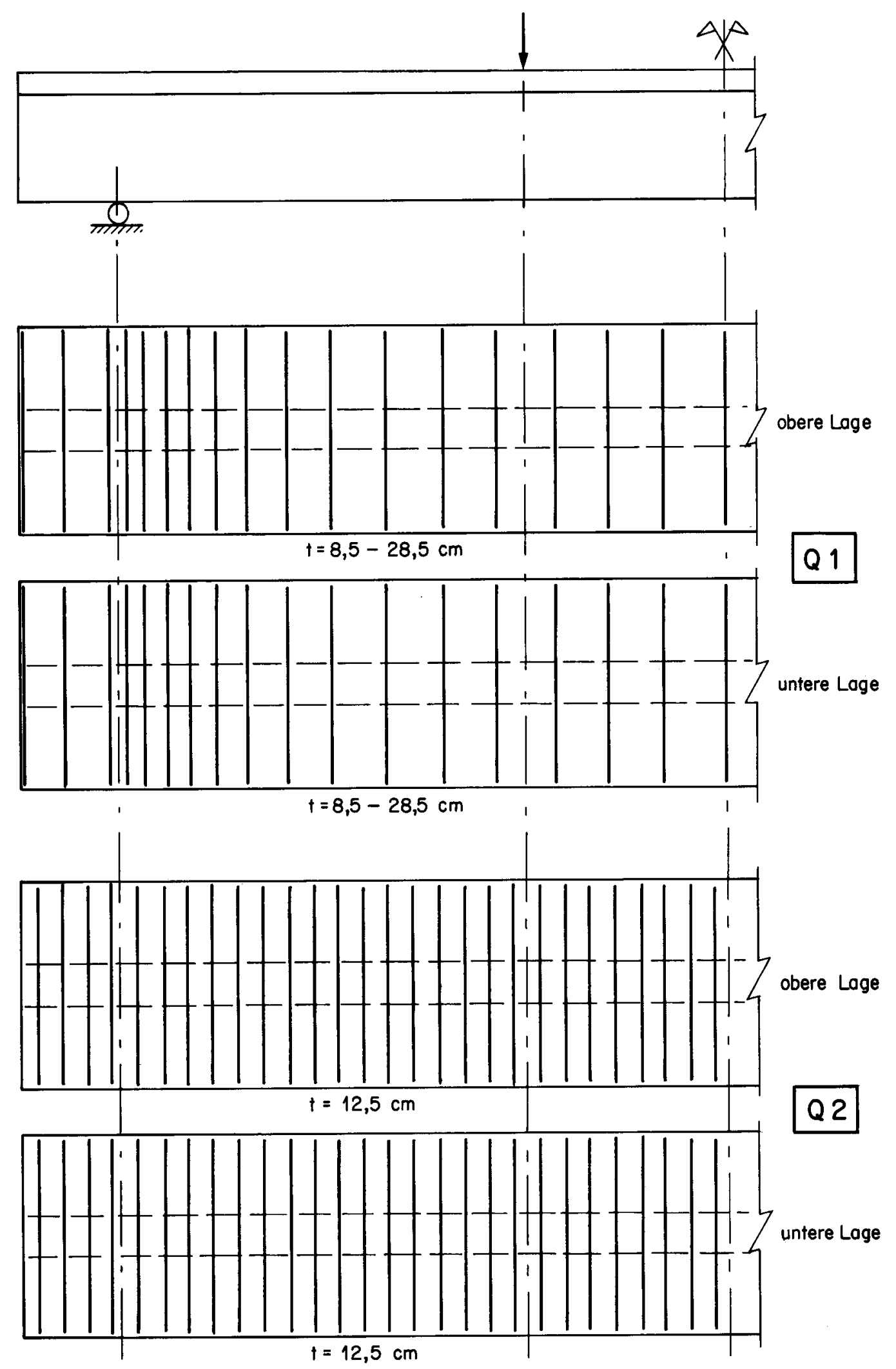

Genaue Eisenabstände siehe Tabelle 8

Bild $11 \mathrm{a}$ : Plattenarmierung $(\phi=6 \mathrm{~mm})$ von $\mathrm{Q} 1, \mathrm{Q} 2$ 

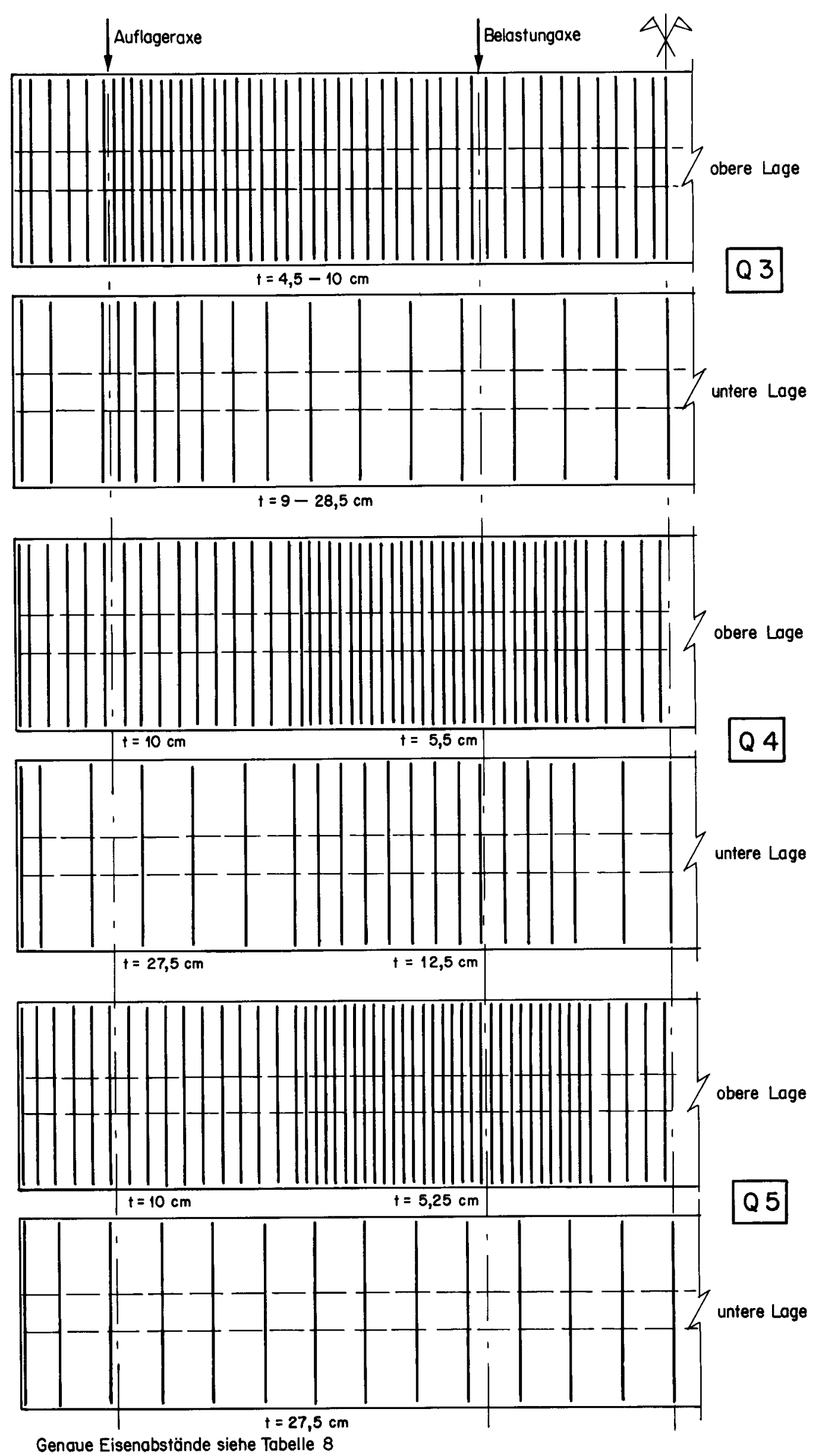

Bild 11b: Plattenarmierung $(\phi=6 \mathrm{~mm})$ von $Q 3, Q 4, Q 5$ 


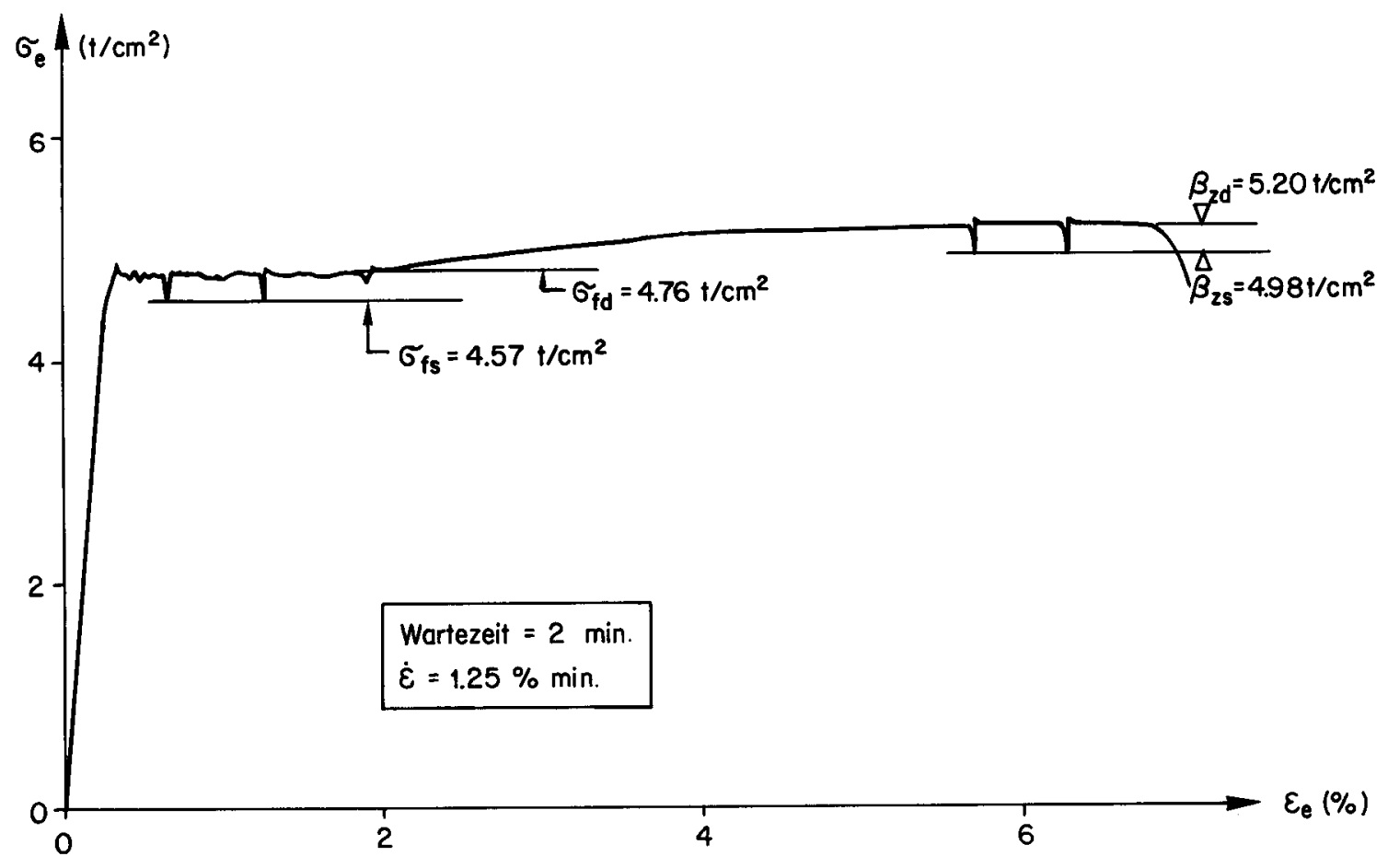

Bild 12: Beispiel für das $\sigma-\varepsilon$-Diagramm des Stahles $\varnothing 6 \mathrm{~mm}$

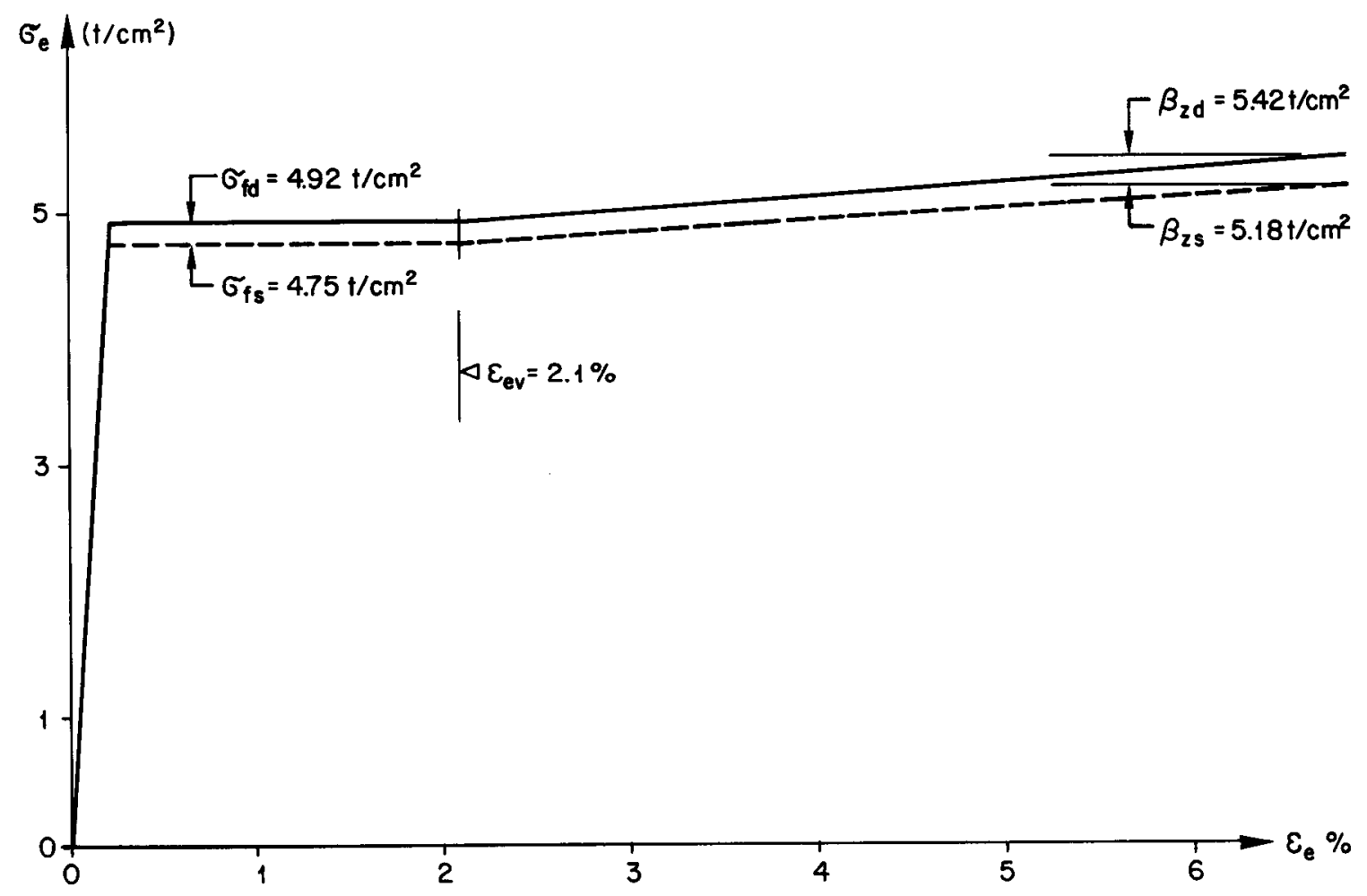

Bild 13 : Gemitteltes idealisiertes $\sigma-\varepsilon$-Diagramm für $\varnothing 6 \mathrm{~mm}$ 


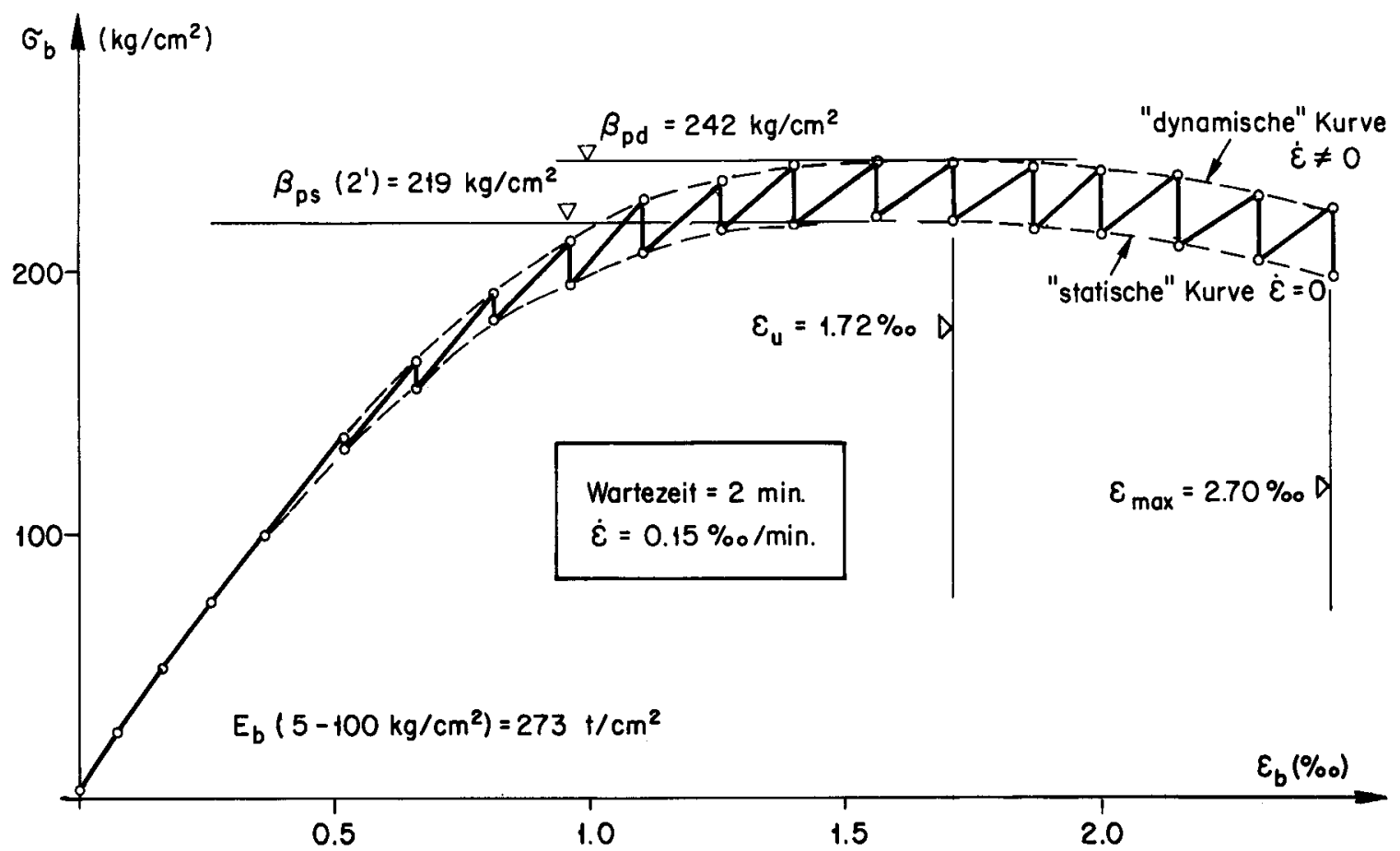

Bild 14: Beispiel für das $\sigma-\varepsilon$-Diagramm eines Betonprismas (Q 5)

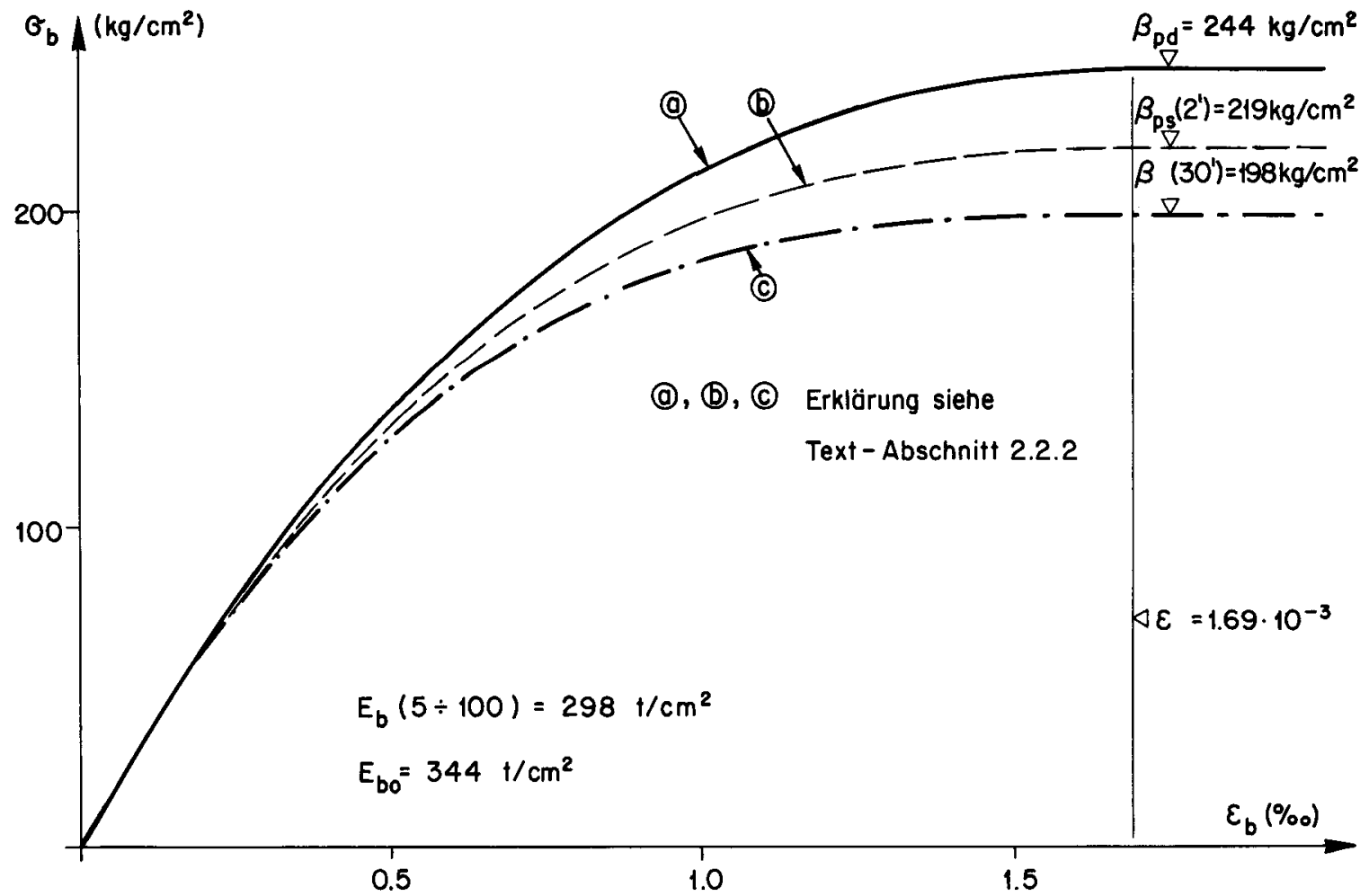

Bild 15: Gemittelte Spannungs-Dehnungs-Diagramme für Auswertung 

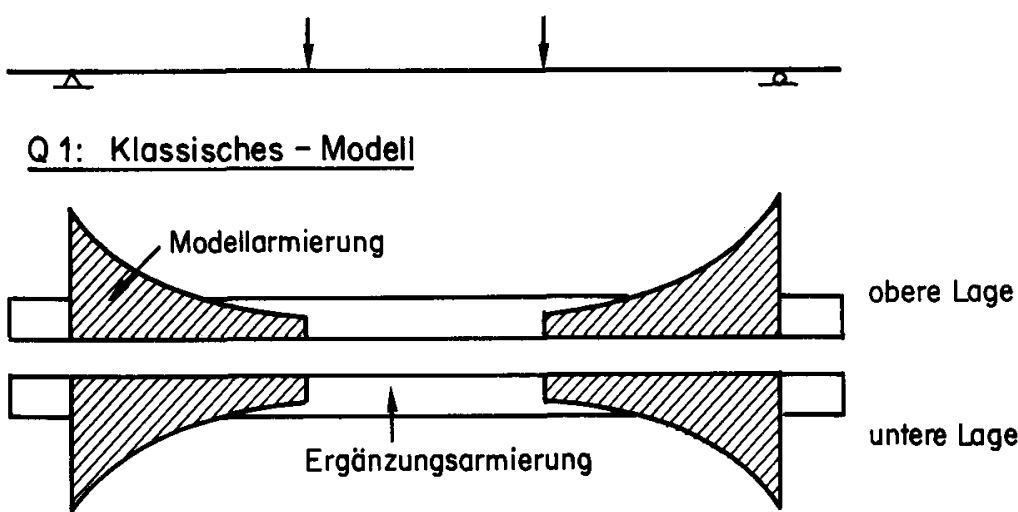

Q2: Fachwerk - Modell

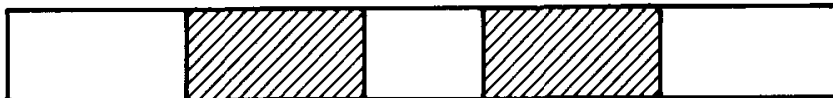

Modell-

ormierung

$\mathrm{cm}^{2}$

Q 1: Klassisches - Modell

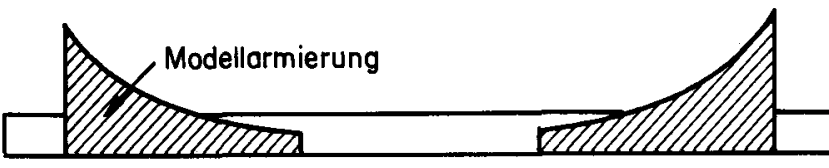

Ergänzungsarmierung

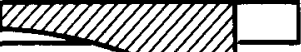

(

untere Lage

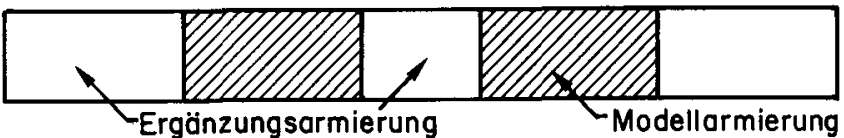

Q3: Superposition der Armierungen "klassisch" und Biegung

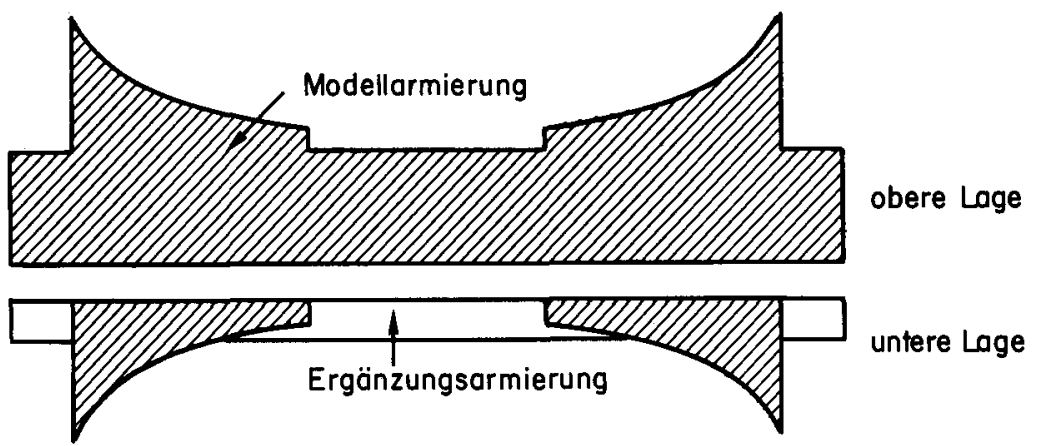

Q4: Superposition der Armierungen "Fachwerk" und Biegung

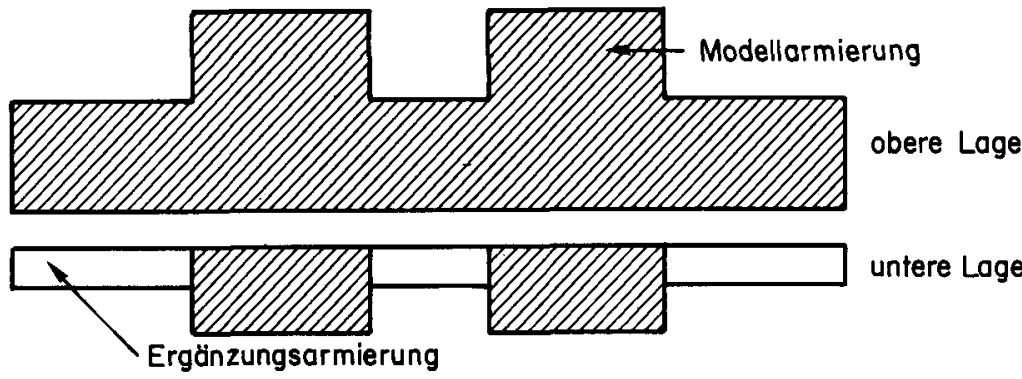

Q5: Biegung mit Axial - Zugkraft "Fachwerk"

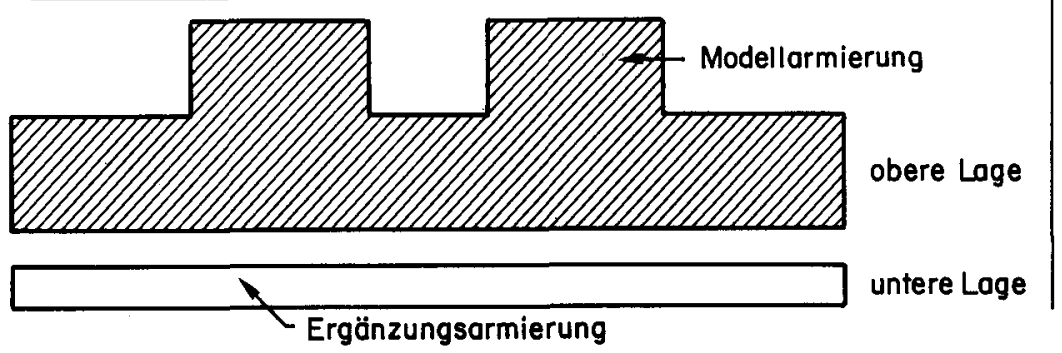
$\mathrm{cm}^{2}$ mierung

Bild 16: Übersicht Modellarmierungen und konstruktive Ergänzungs armierungen 
Loststellung $P$ für Löngsbeanspruchung bei $Q_{1}-Q_{5}$ :

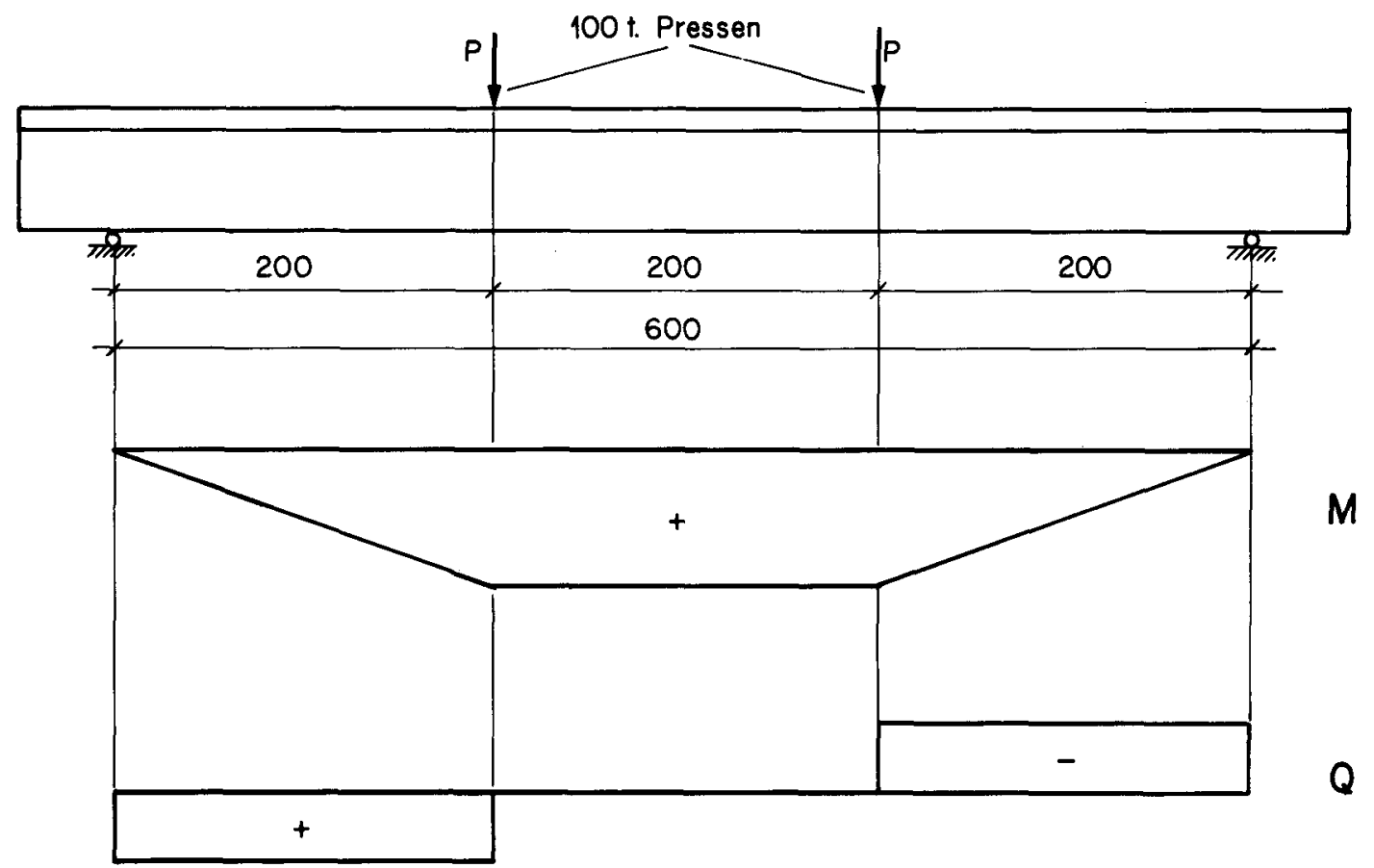

Laststellung $\bar{P}$ für Querbeanspruchung bei $Q_{3}-Q_{5}$ :
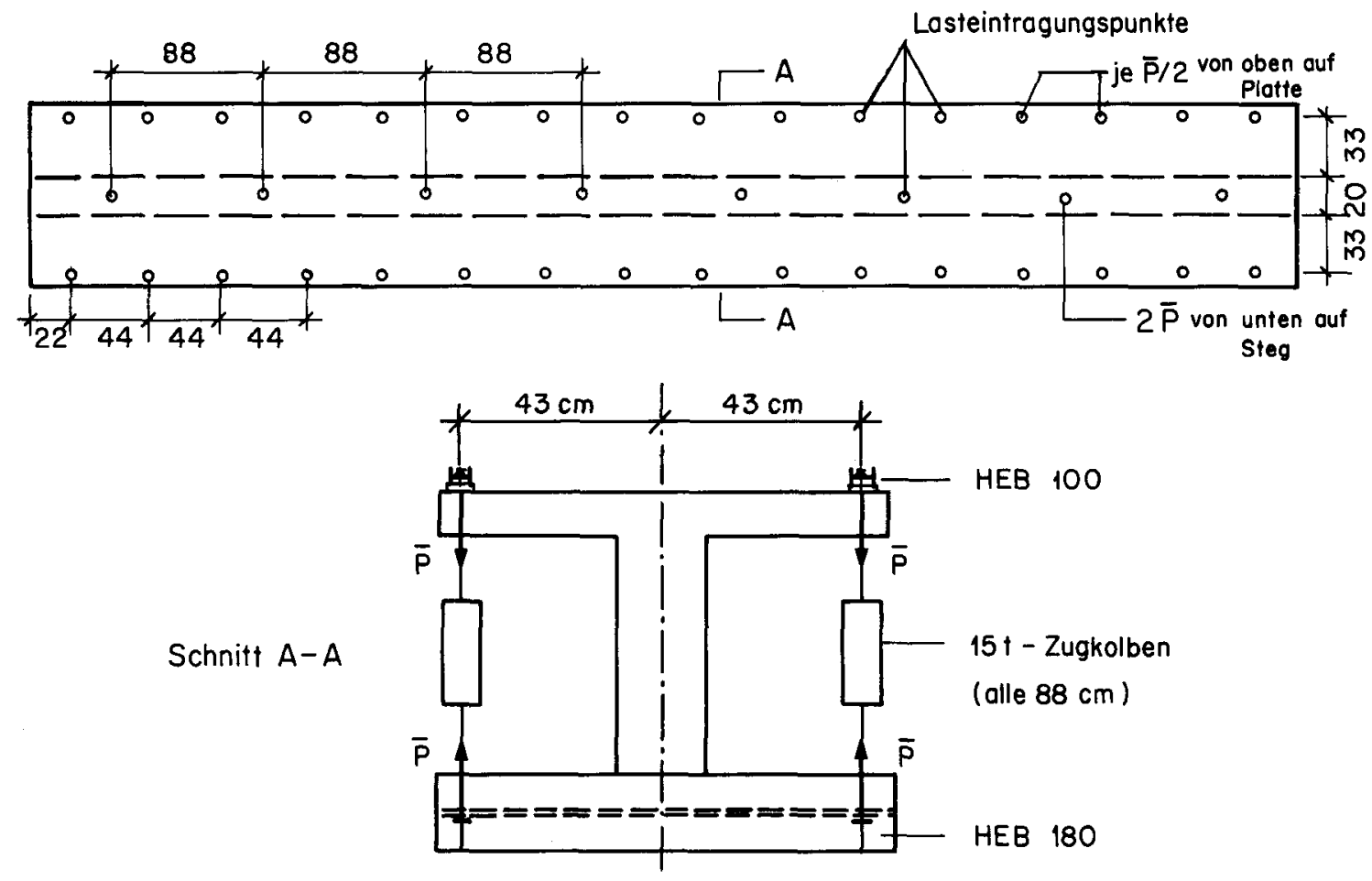

Bild 17: Belastungsanordnung für Längs - und Querbeanspruchung 


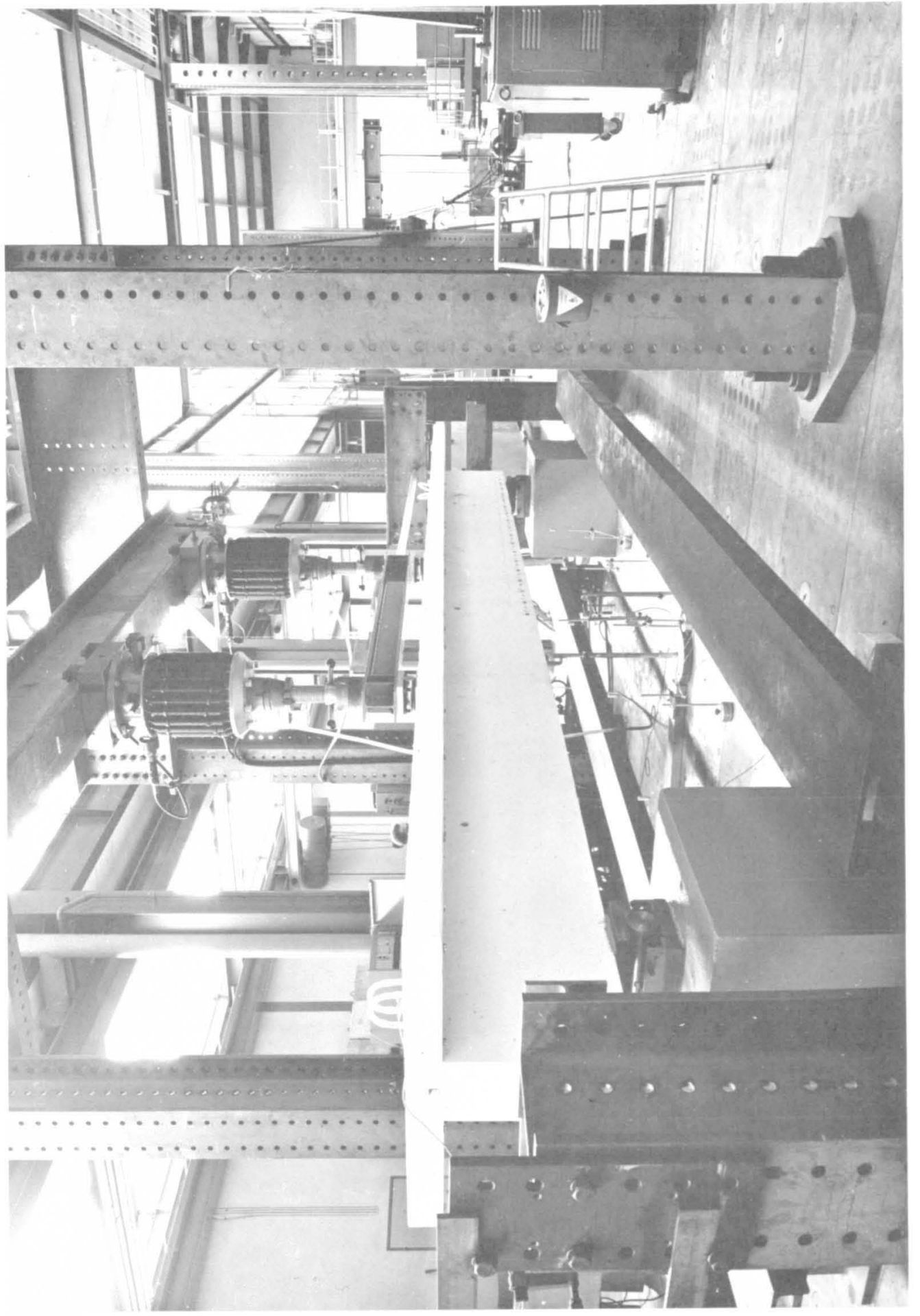

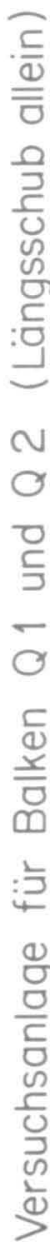




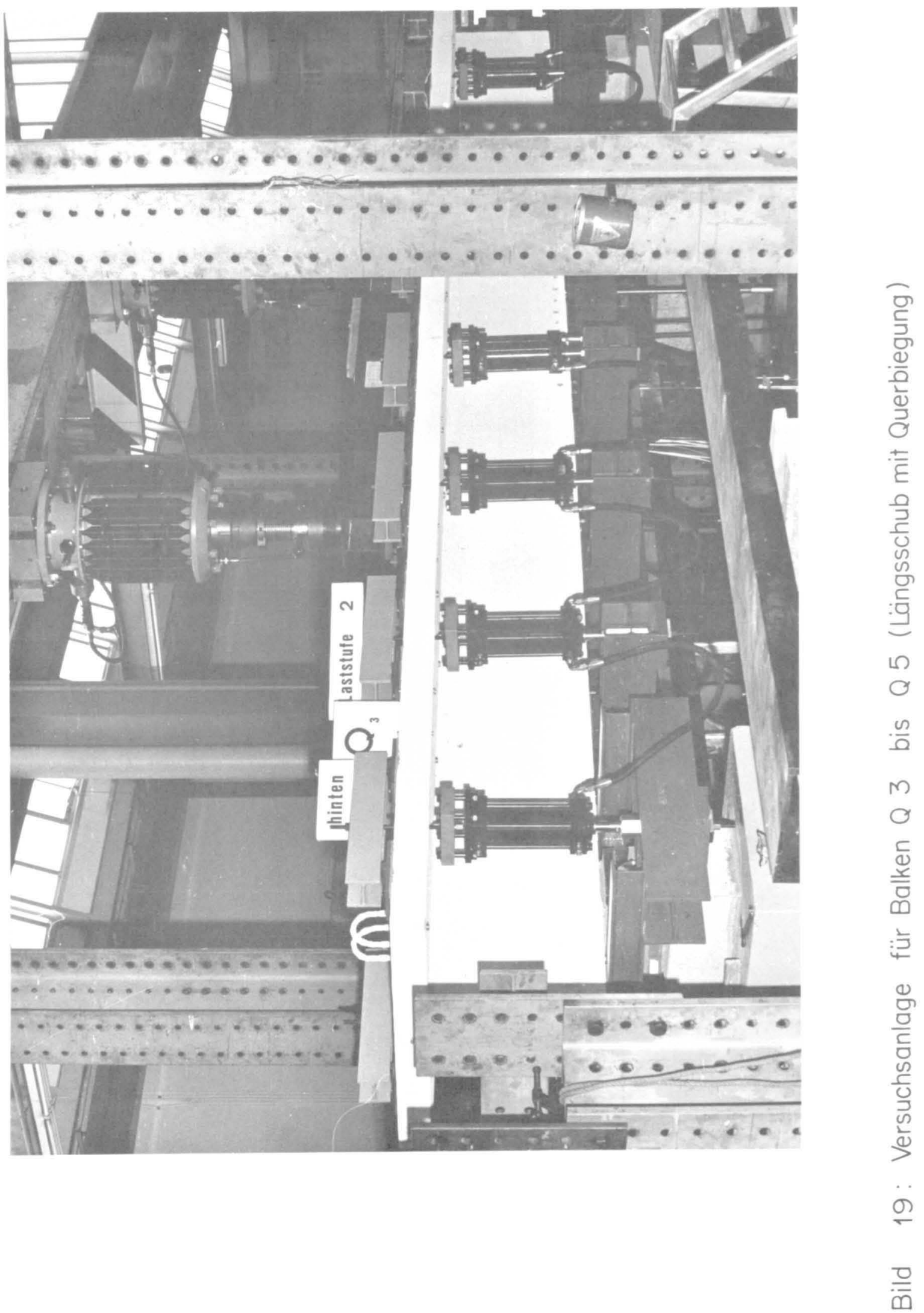



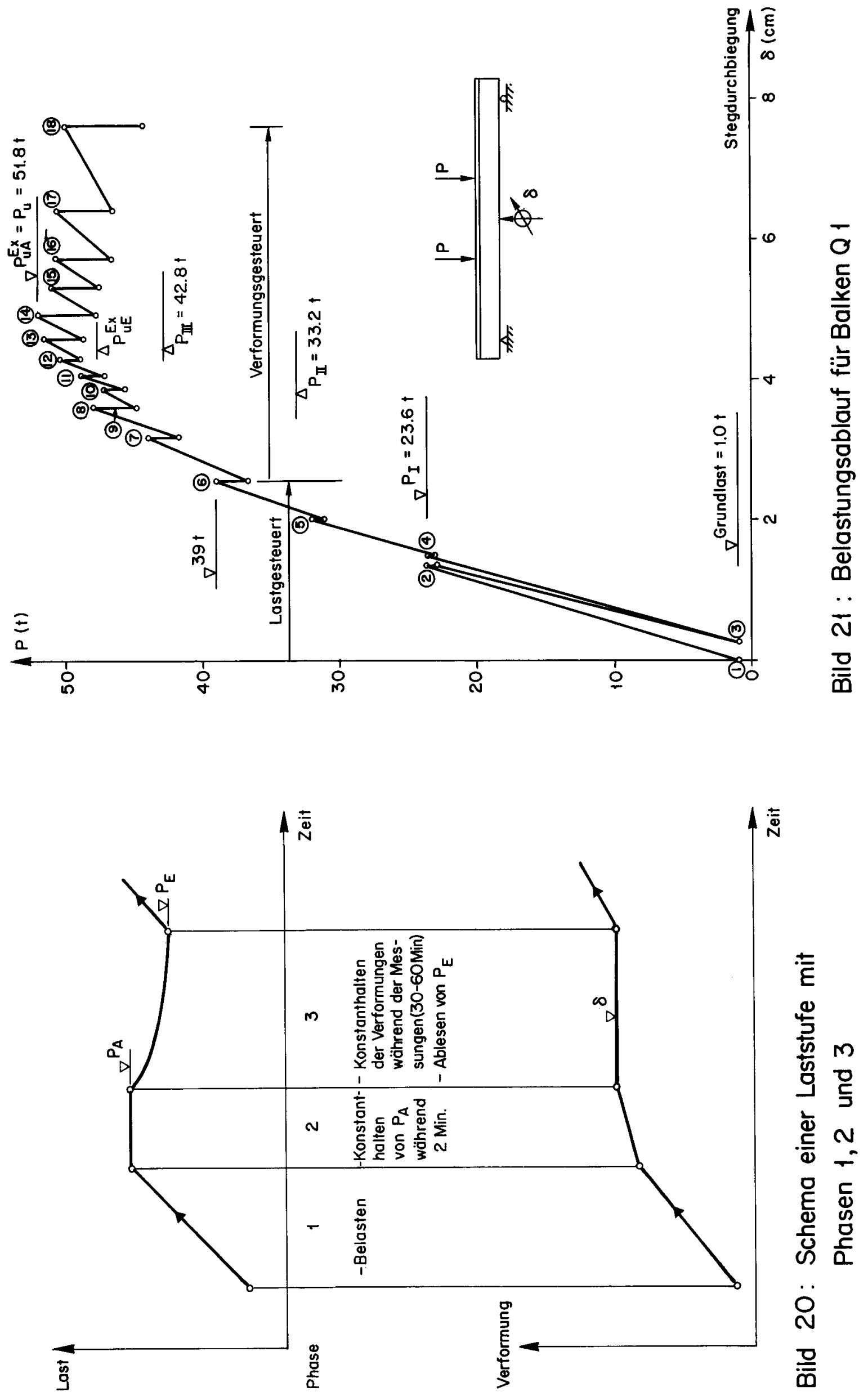

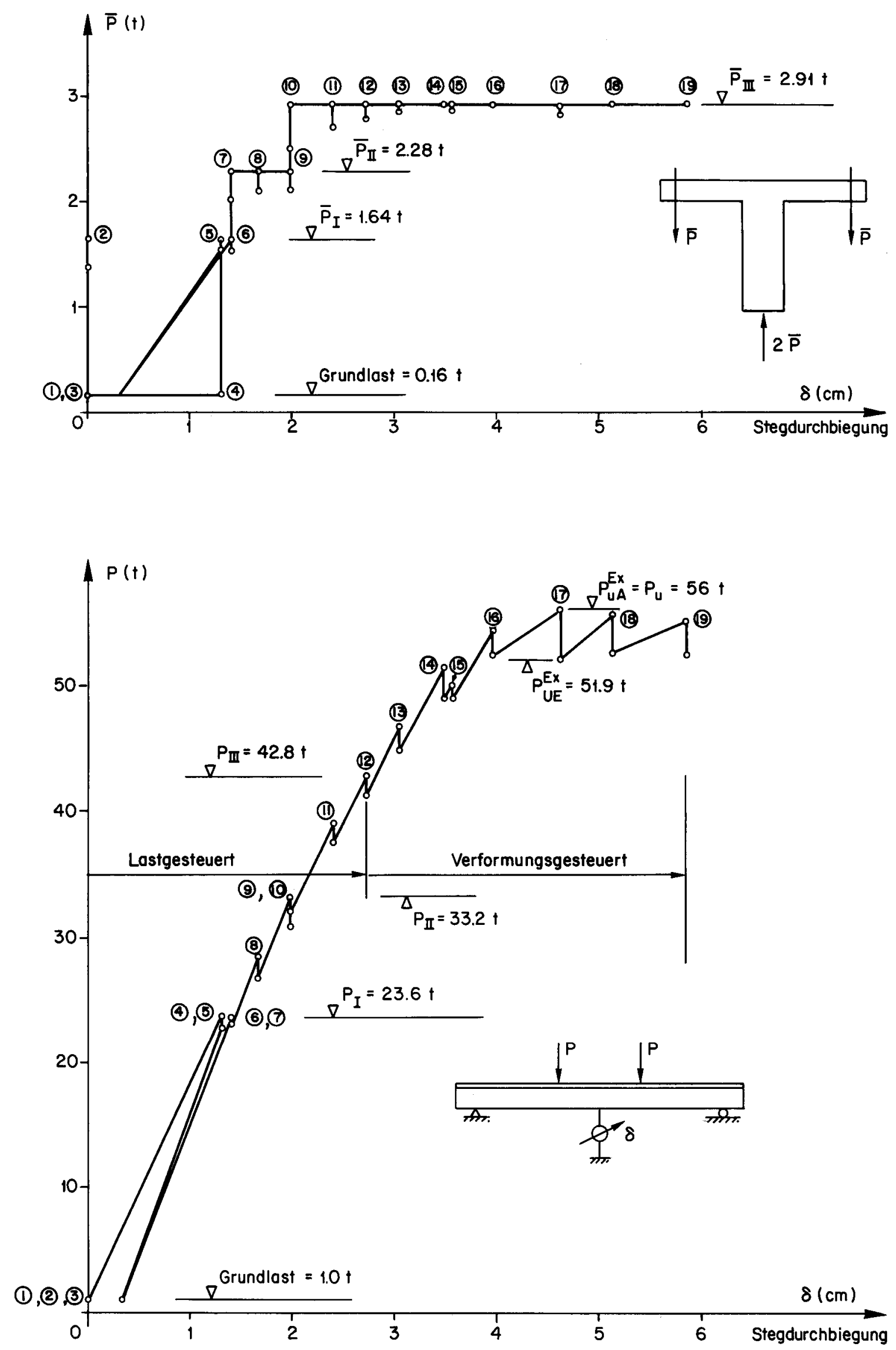

Bild 22: Belastungsablauf für Balken Q 4 

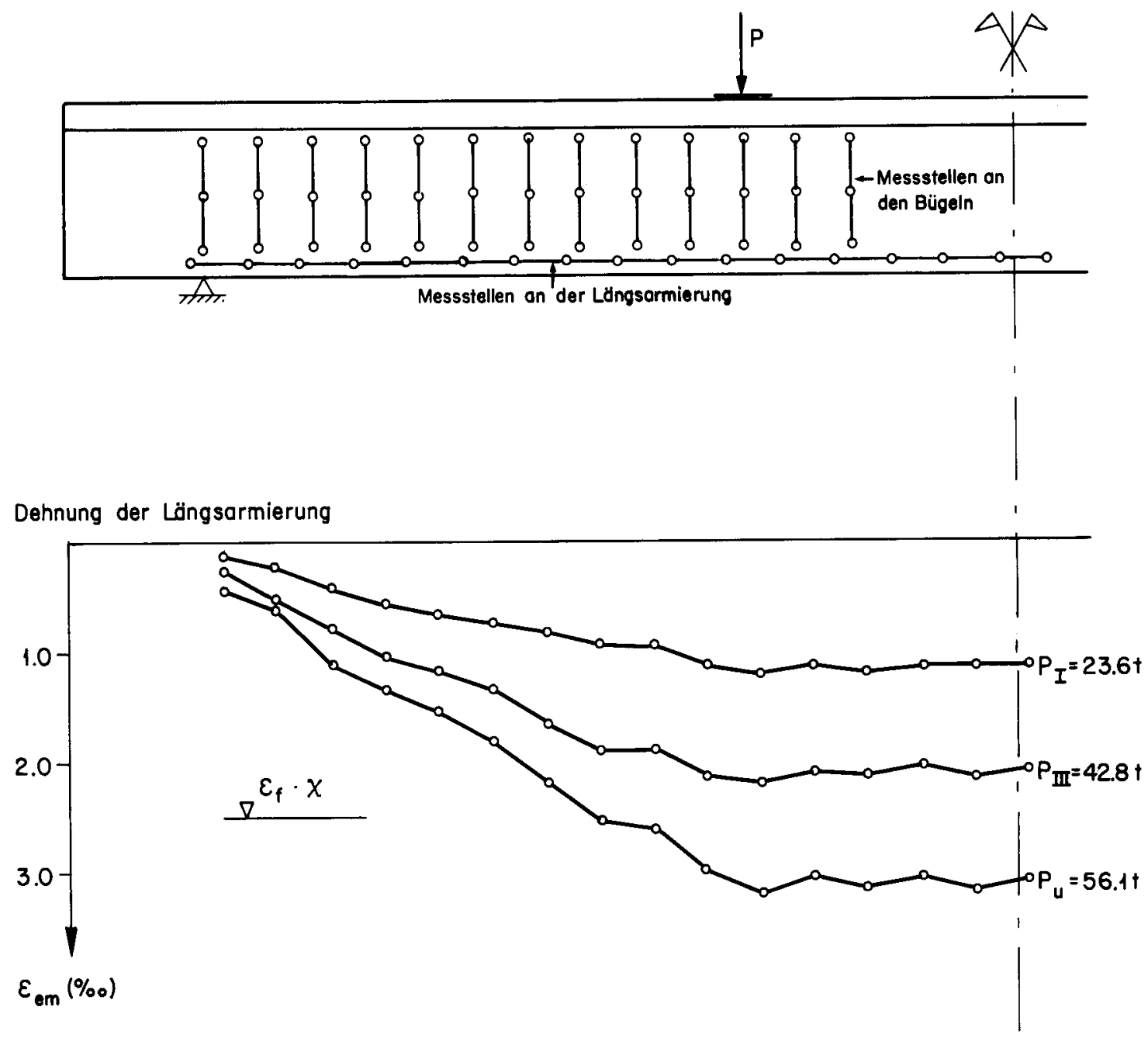

Dehnung der Bügelarmierung

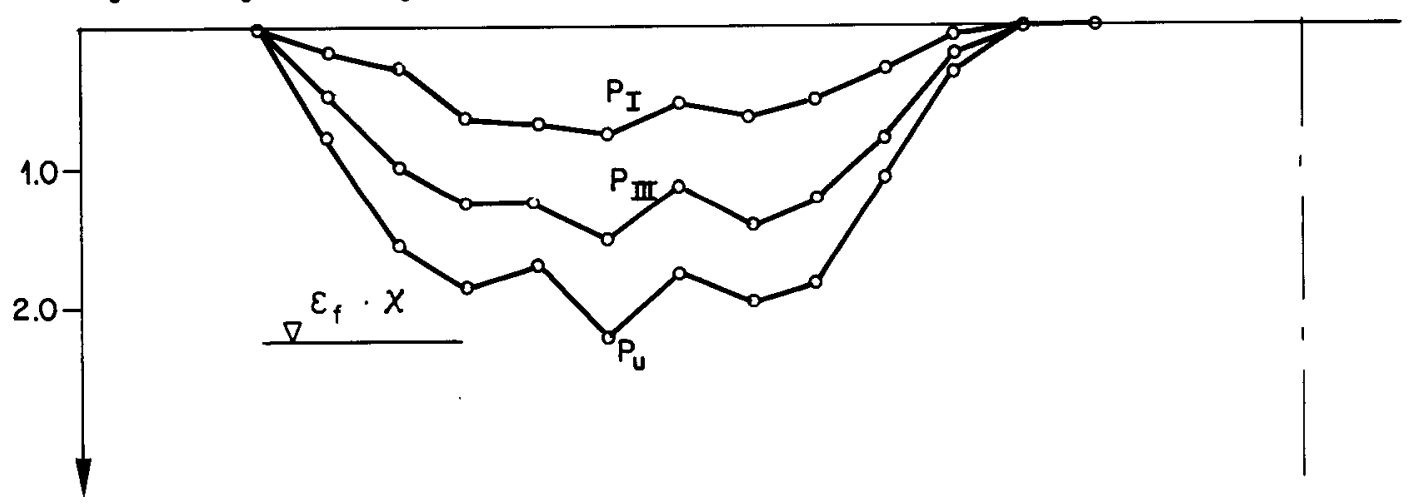

$\varepsilon_{\text {em }}(\% \circ)$

Bild 23: Beispiel für Beanspruchung der Stegarmierung (Balken Q 2) 
Stegdurchbiegung $\delta_{s}$
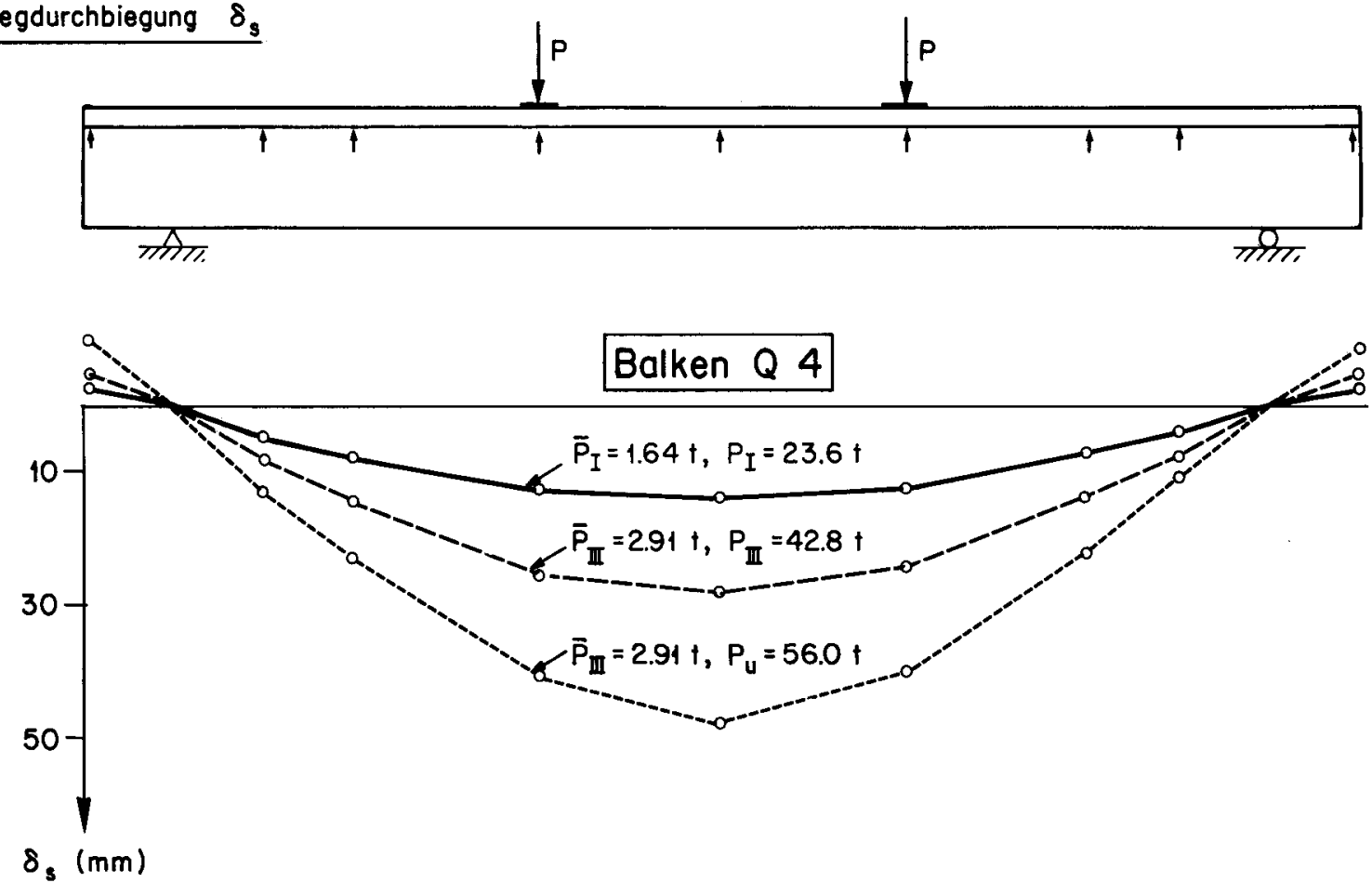

Relative Plattendurchbiegung $\delta_{r}$
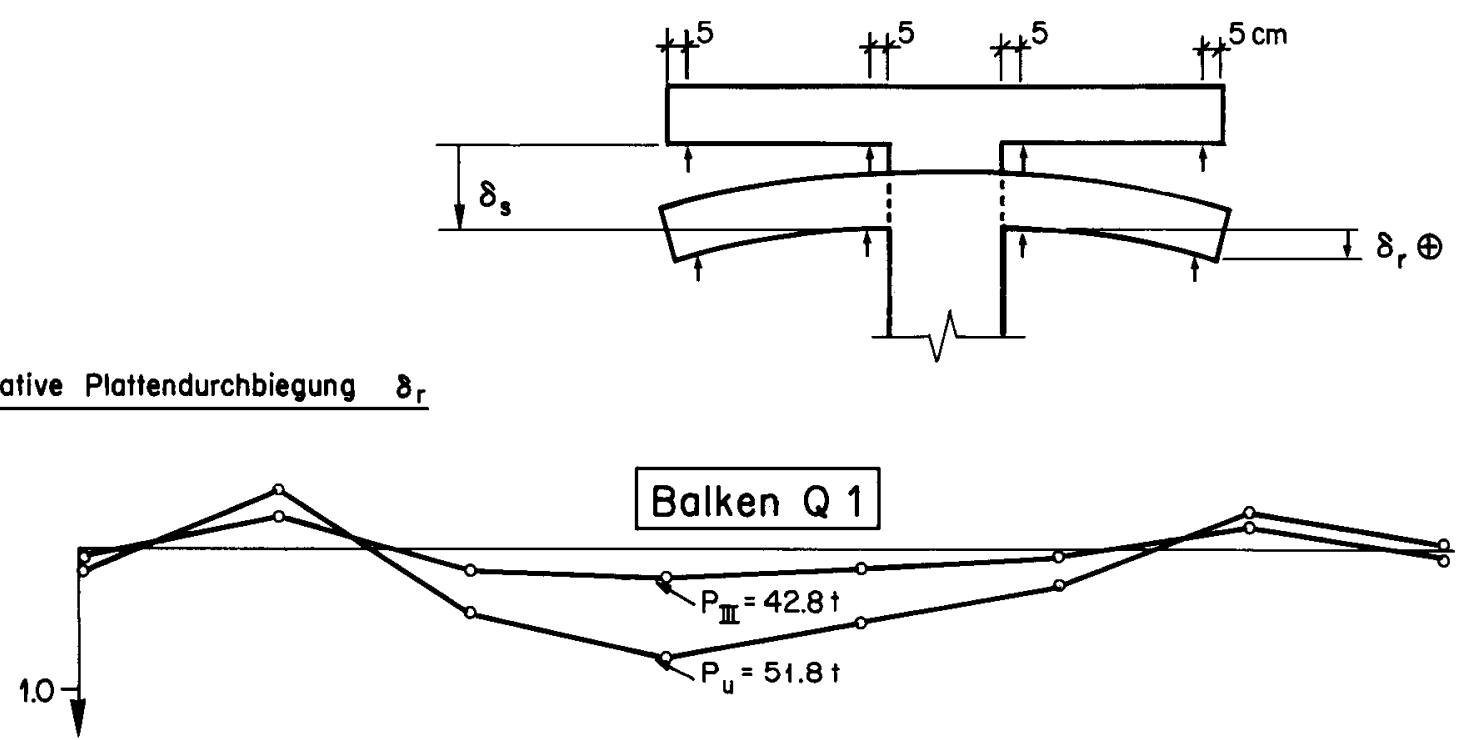

$\delta_{r}(\mathrm{~mm})$

Balken Q 4

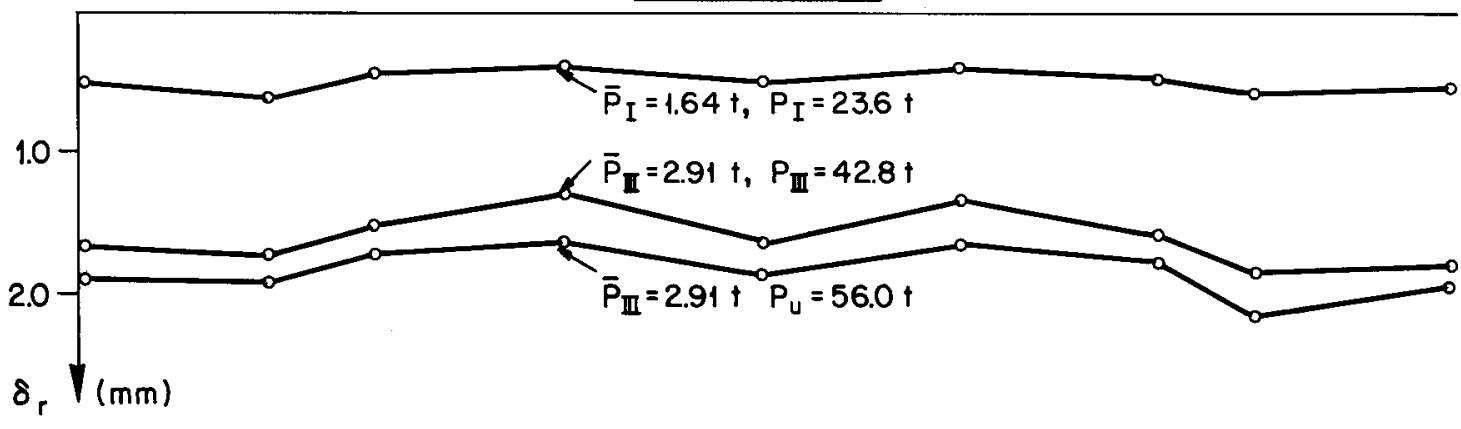

Bild 24: Beispiele für die Durchbiegungen längs der Balken 

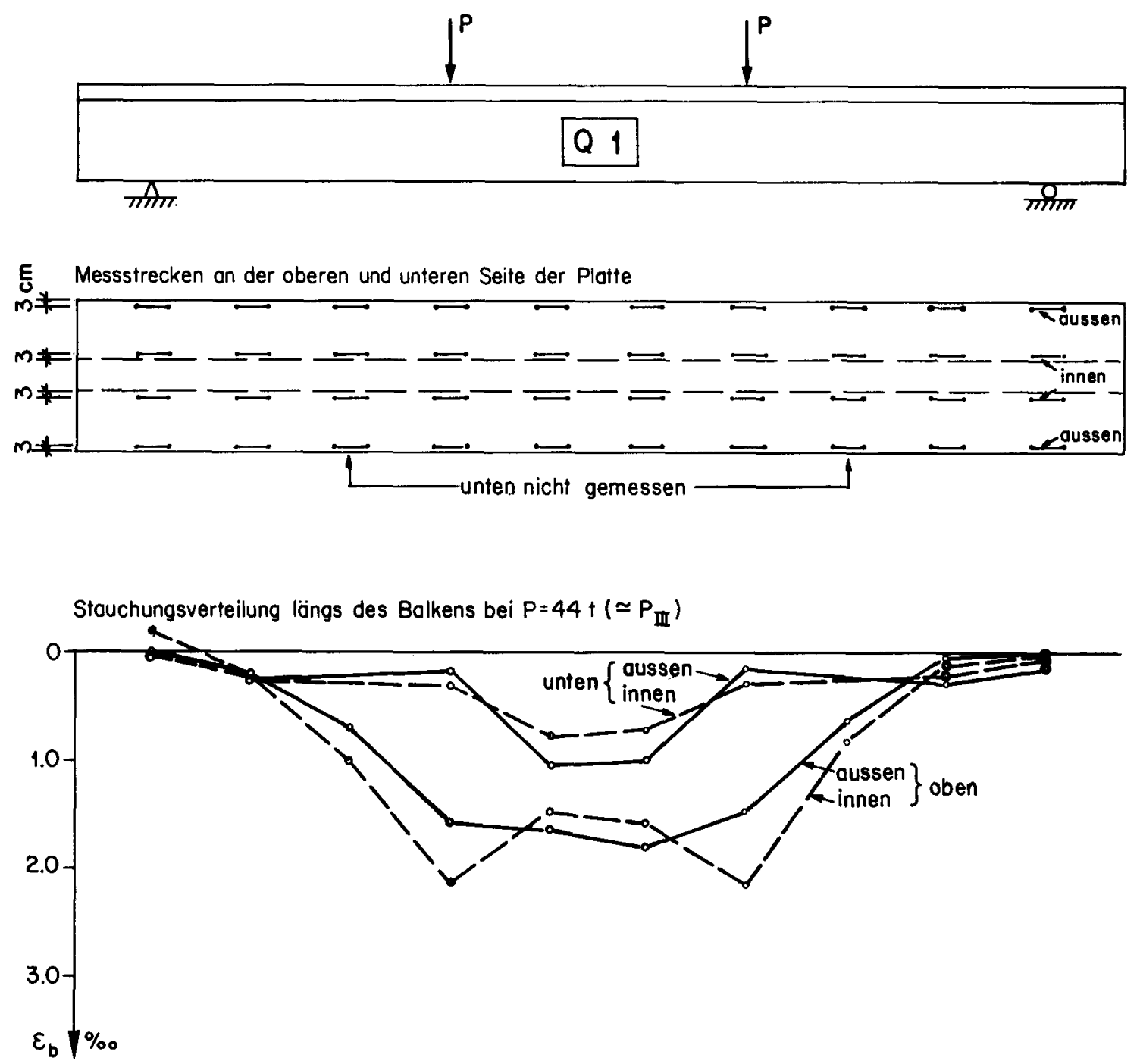

Stauchungsverteilung längs des Balkens bei $P_{u}=51.8 \dagger$

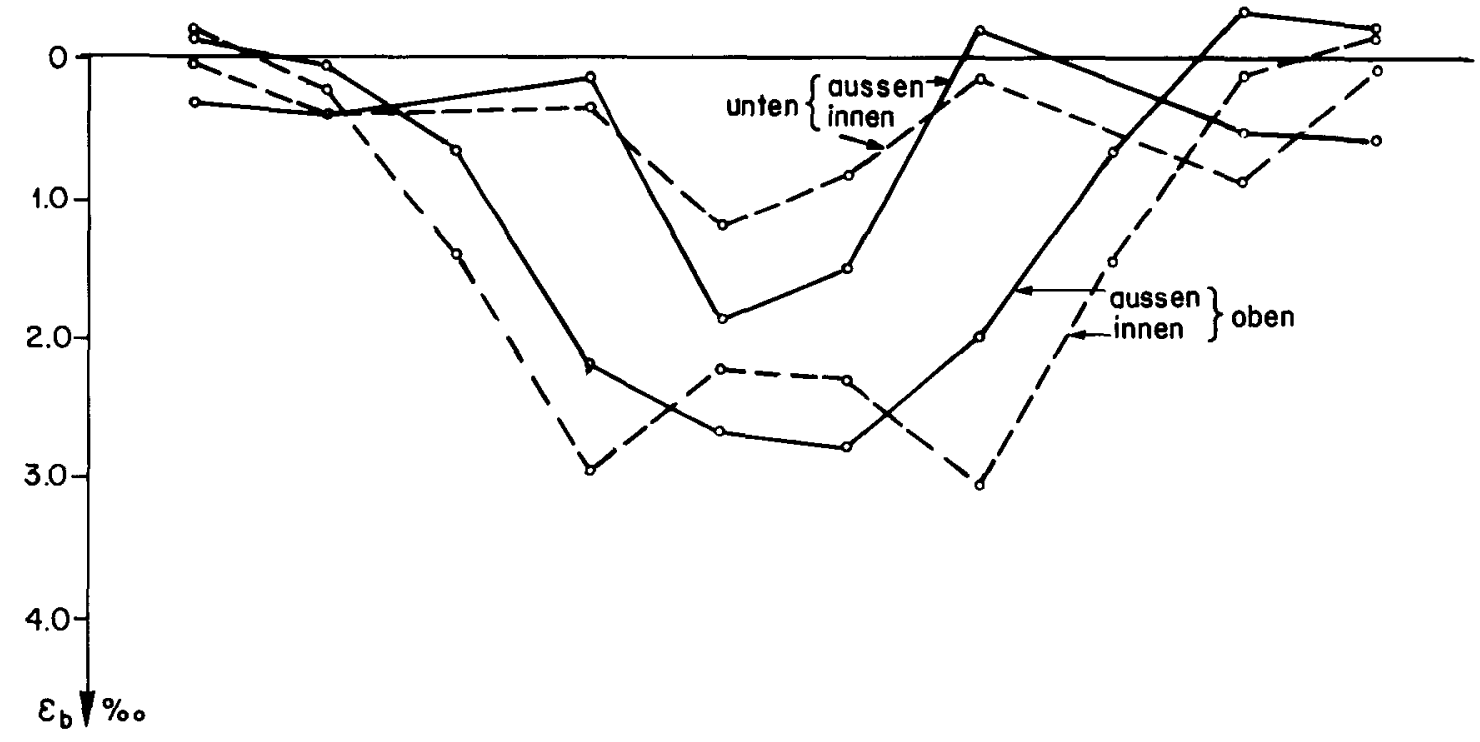

Bild 25: Betonstauchung der Platte bei Balken Q 1 

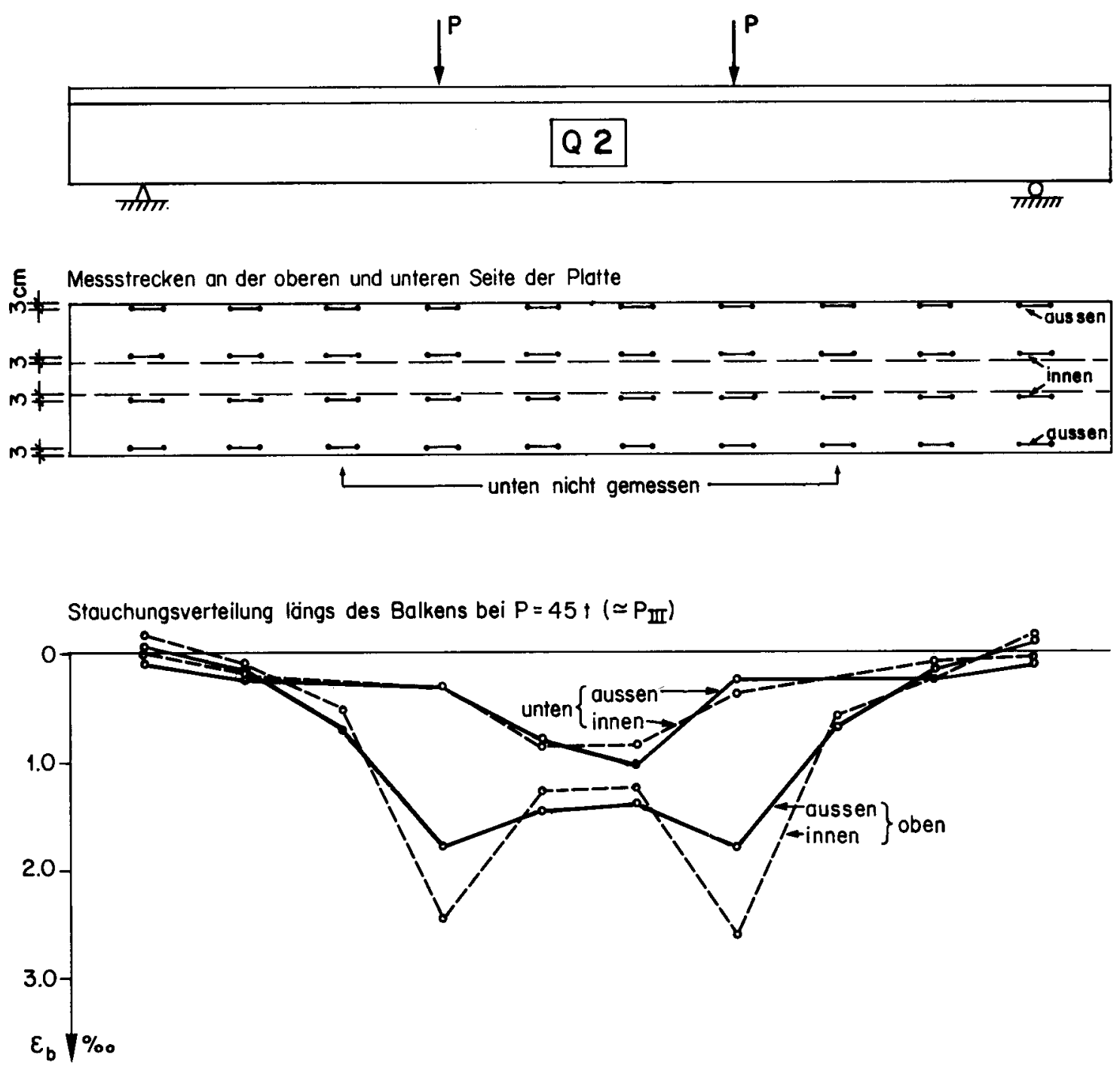

Stauchungsverteilung längs des Balkens bei Euststufe 16, $P=56+\left(\simeq P_{u}\right)$

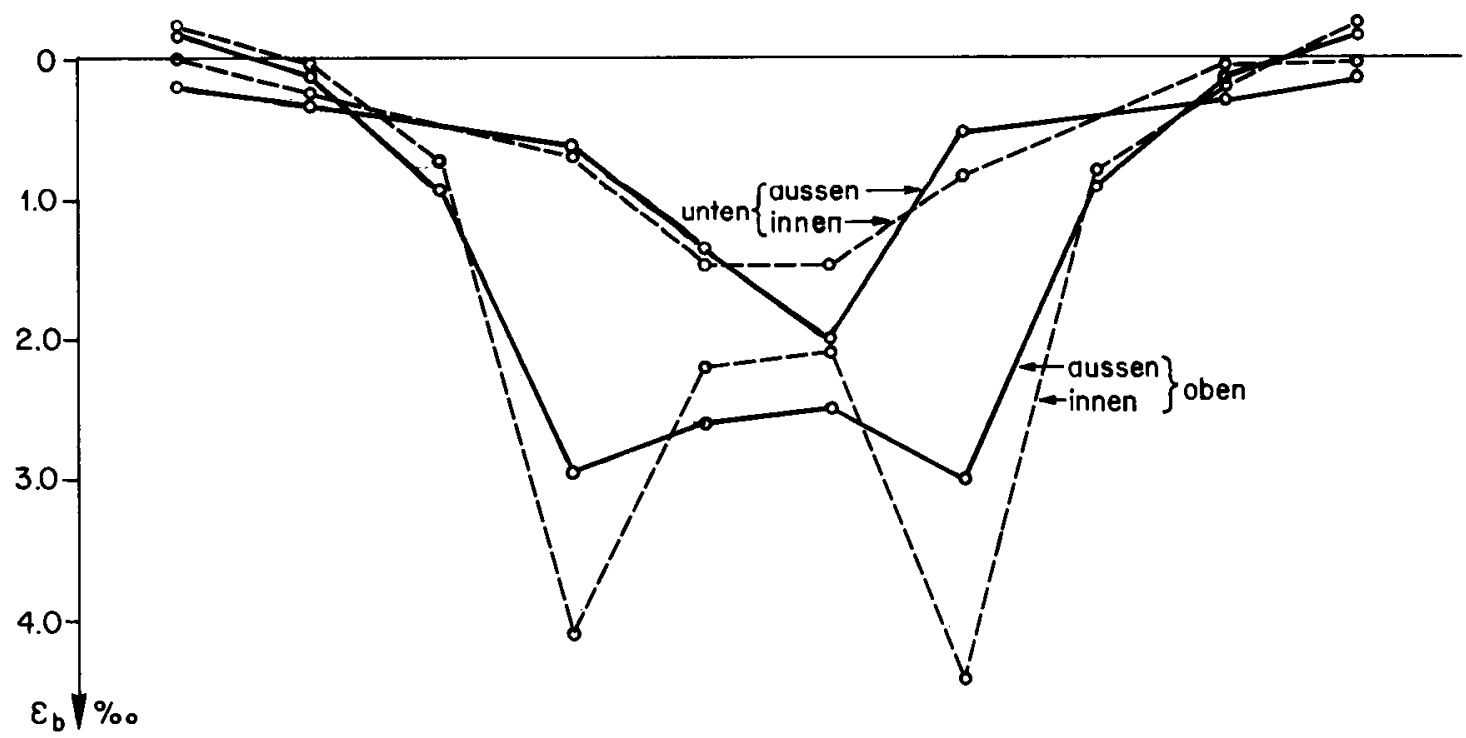

Bild 26: Betonstauchung der Platte bei Balken Q 2 

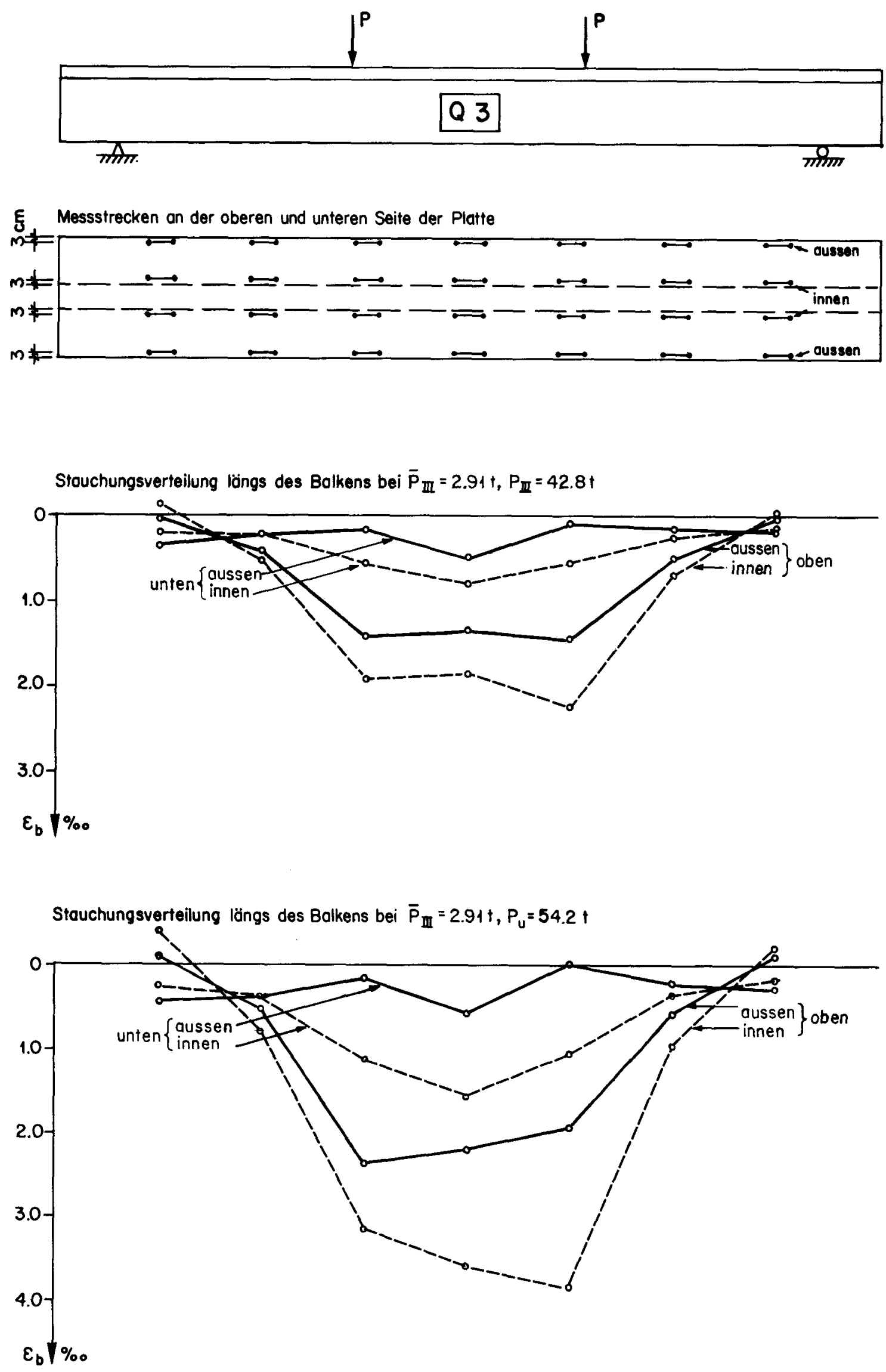

Bild 27: Betonstauchung der Platte bei Balken Q 3 

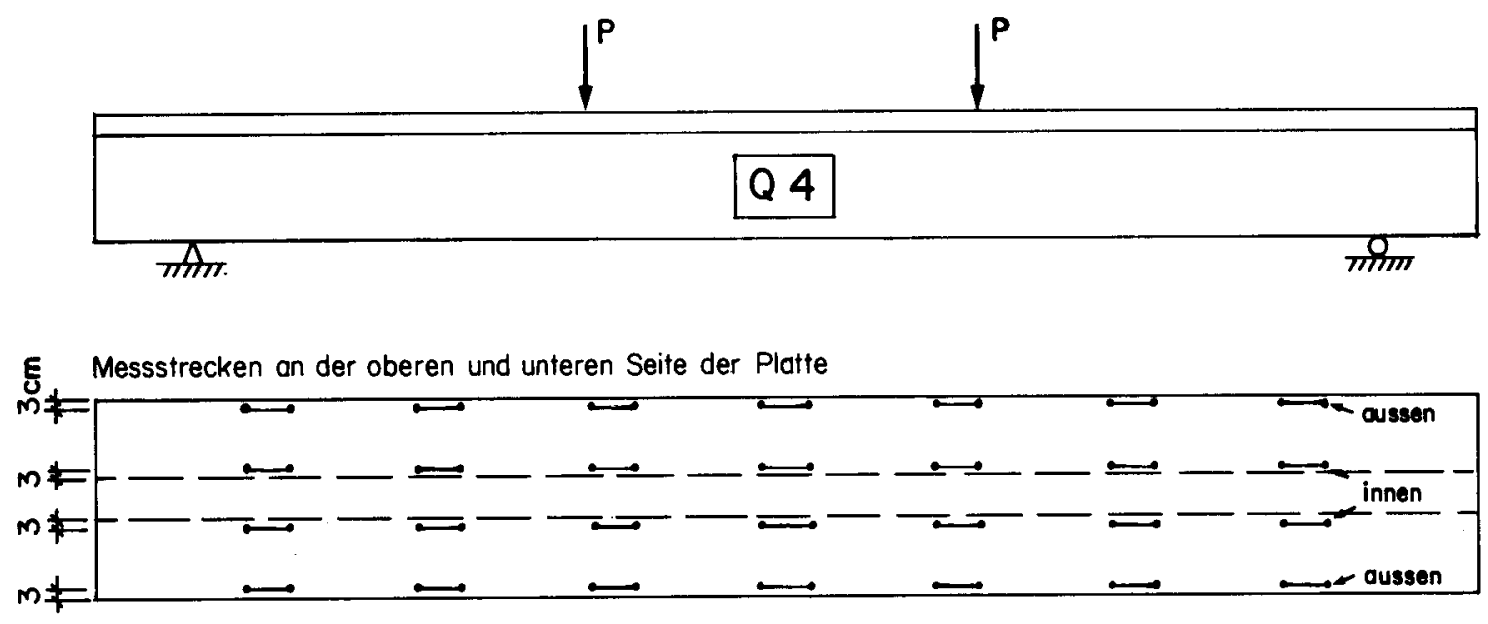

Stauchungsverteilung löngs des Balkens bei $\bar{P}_{\mathbb{I I}}=2.91 \uparrow, P_{\mathbf{I}}=42.8 \dagger$

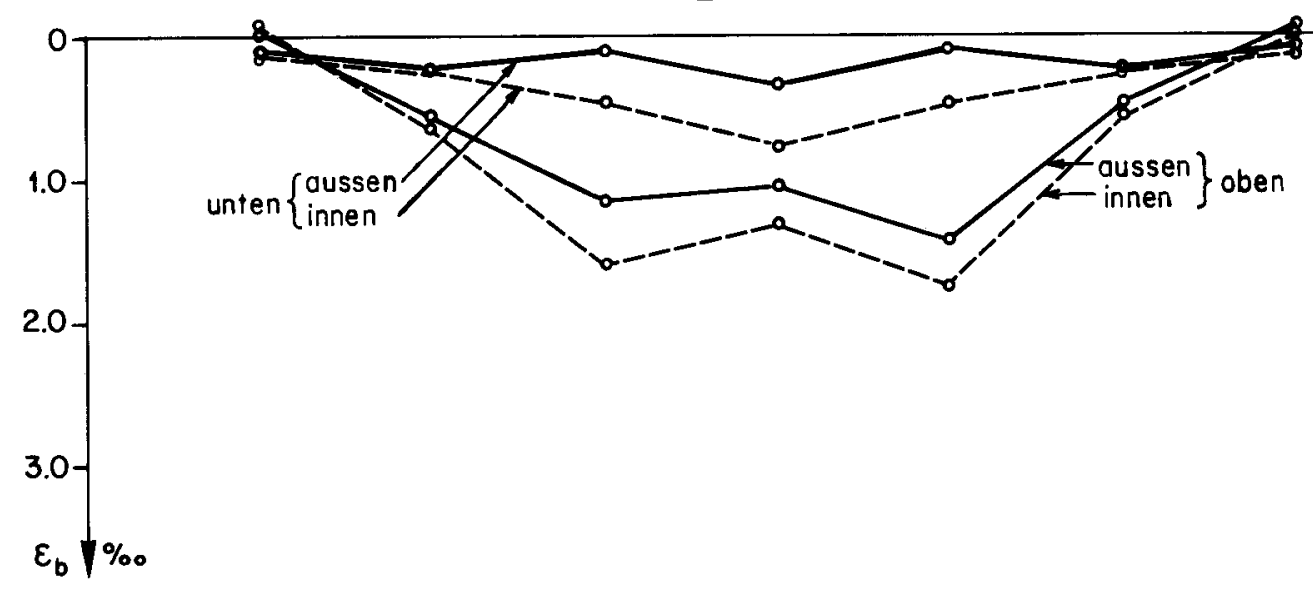

Stauchungsverteilung längs des Balkens bei $\bar{P}_{\text {III }}=2.91 \uparrow, P_{U}=56 \uparrow$

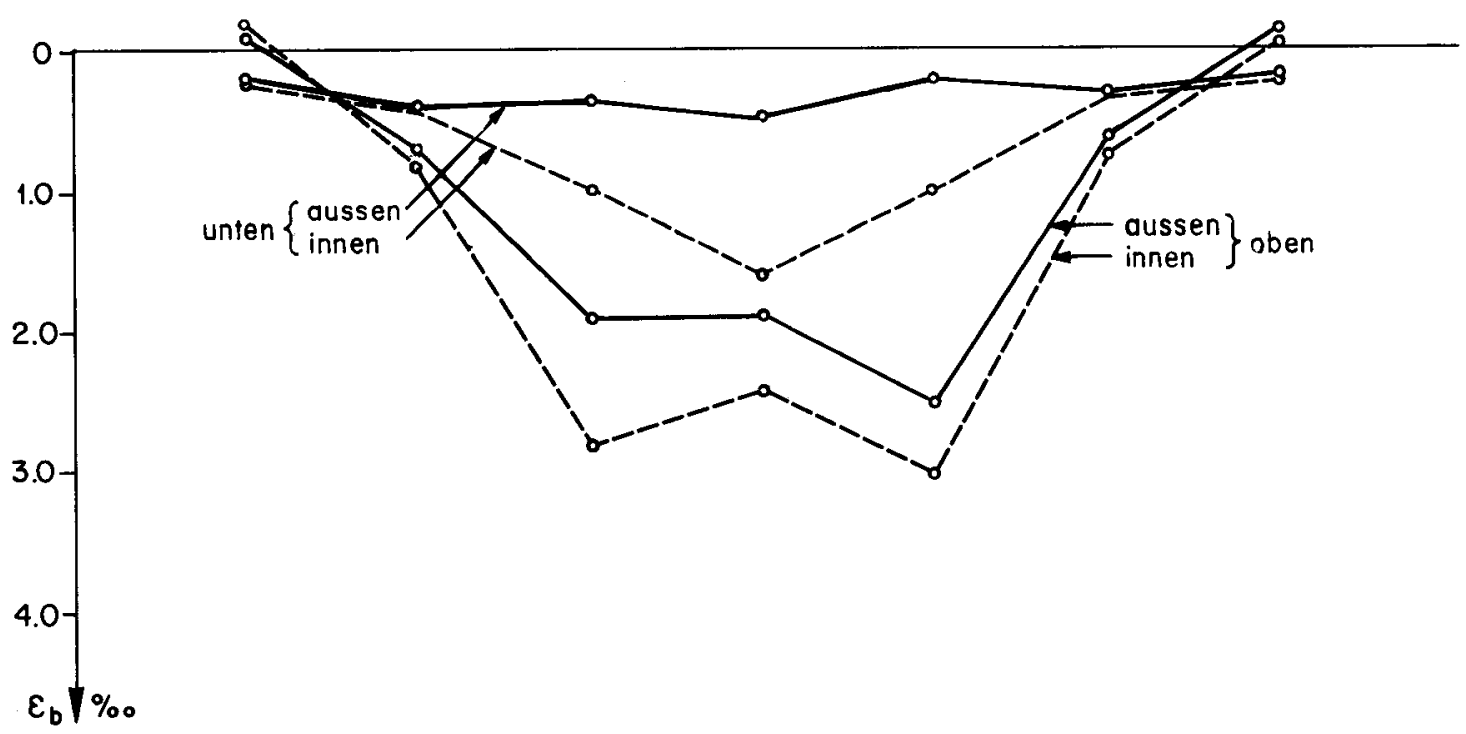

Bild 28: Betonstauchung der Platte bei Balken Q 4 

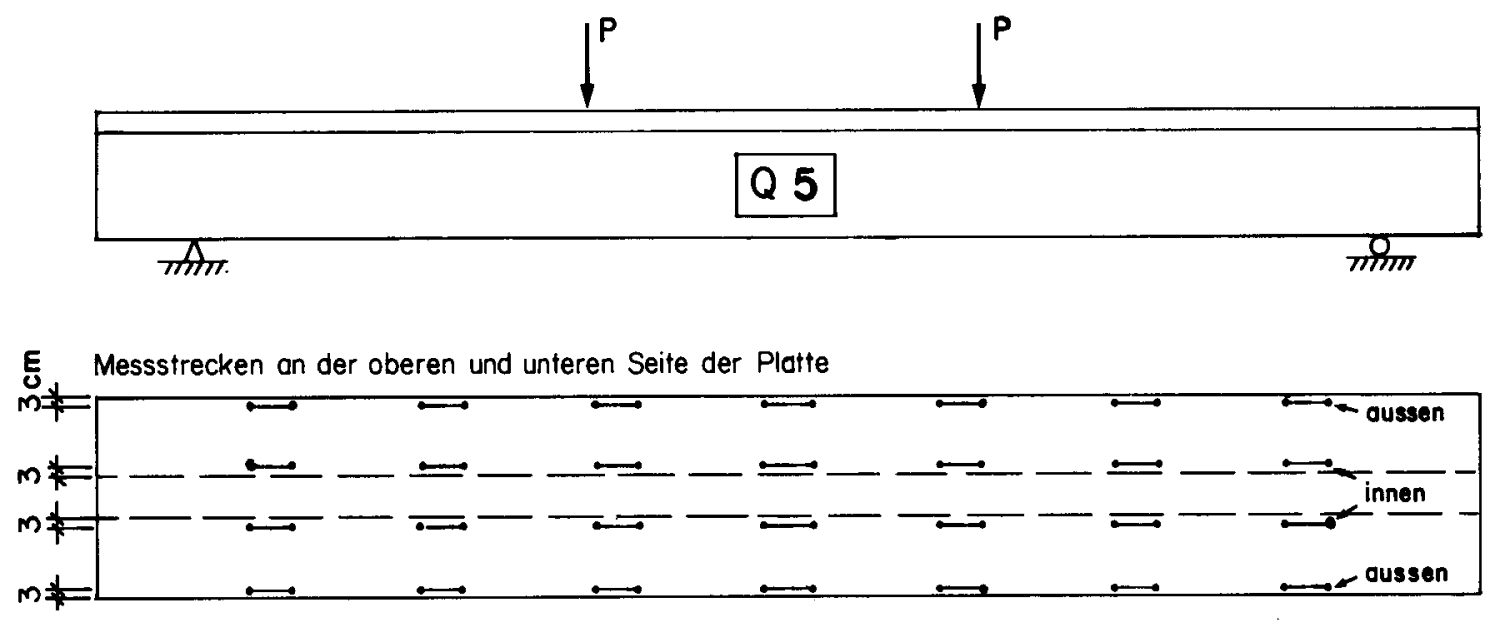

Stauchungsverteilung längs des Balkens bei $\bar{P}_{\text {II }}=2.91 \mathrm{t}, P_{\text {III }}=42.8 t$

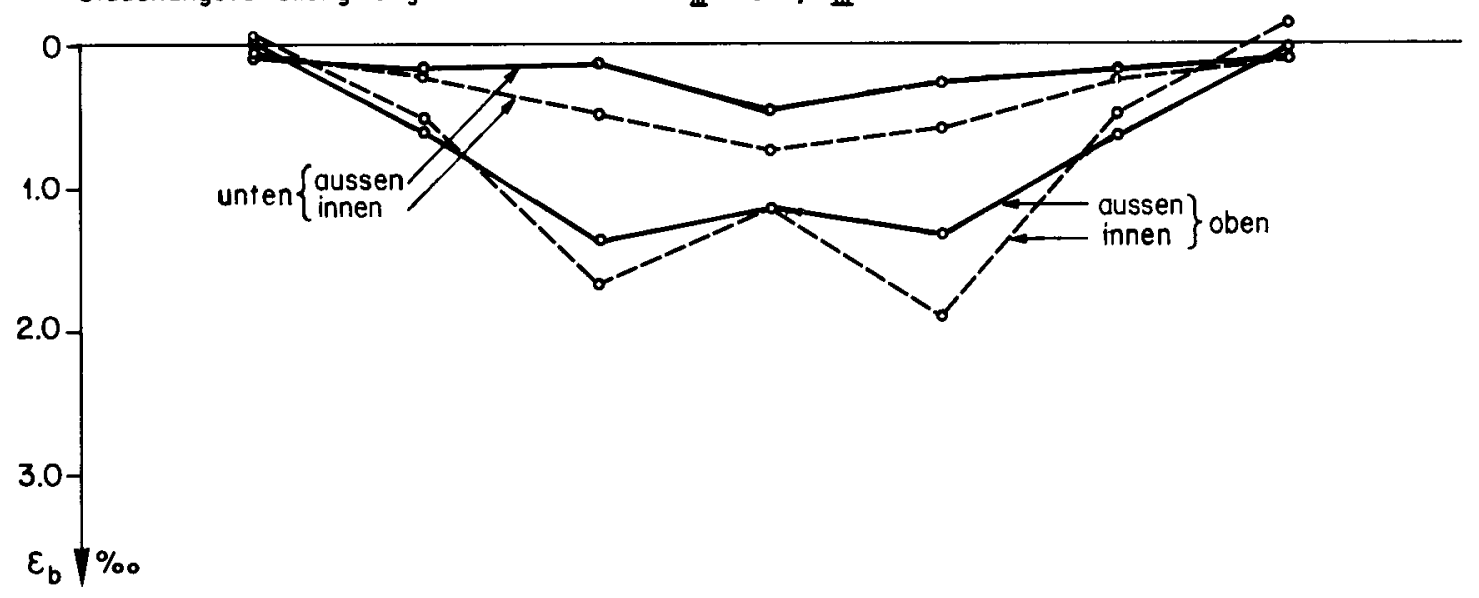

Stauchungsverteilung längs des Balkens bei $\bar{P}_{\text {III }}=2.91 \dagger, P_{U}=52.3 \dagger$

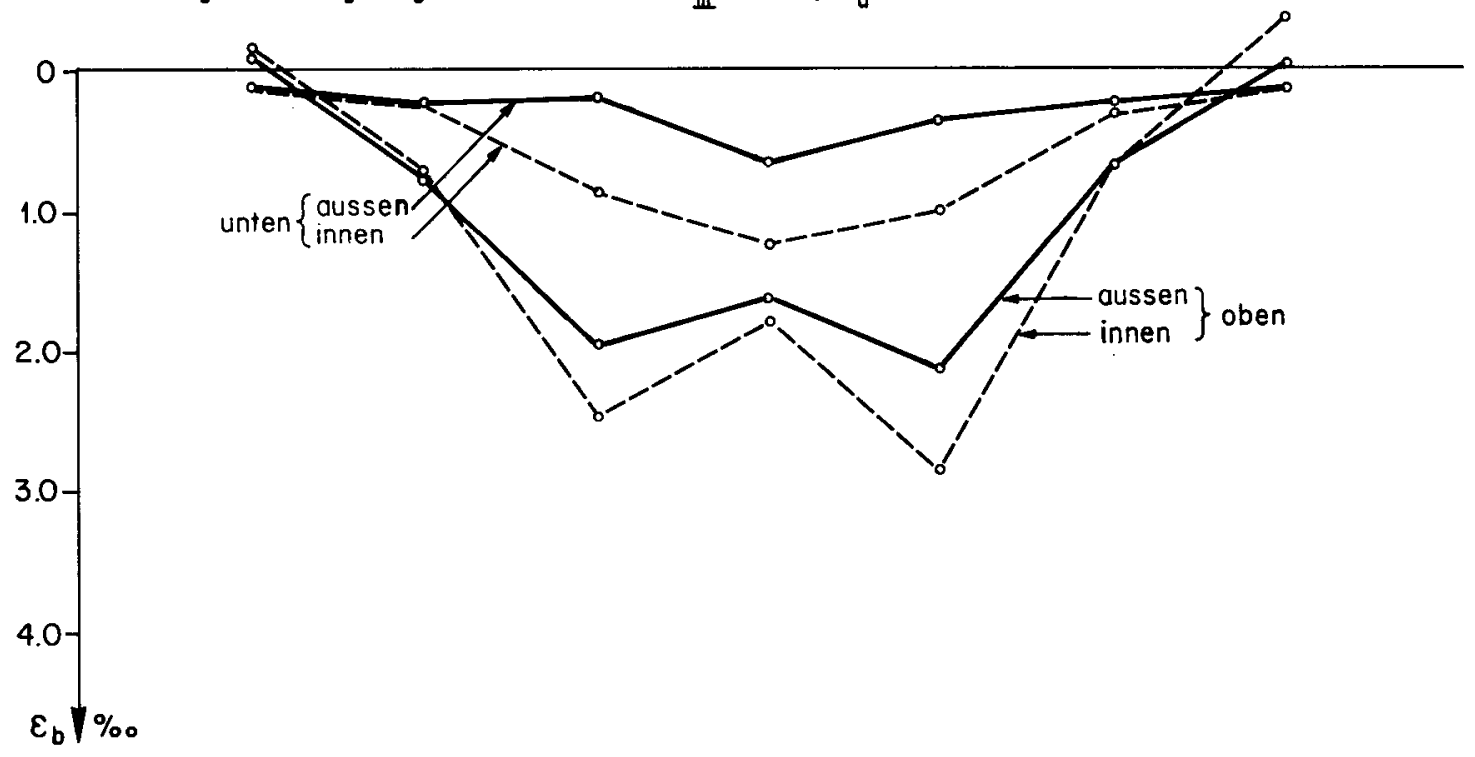

Bild 29: Betonstauchung der Platte bei Balken Q 5 

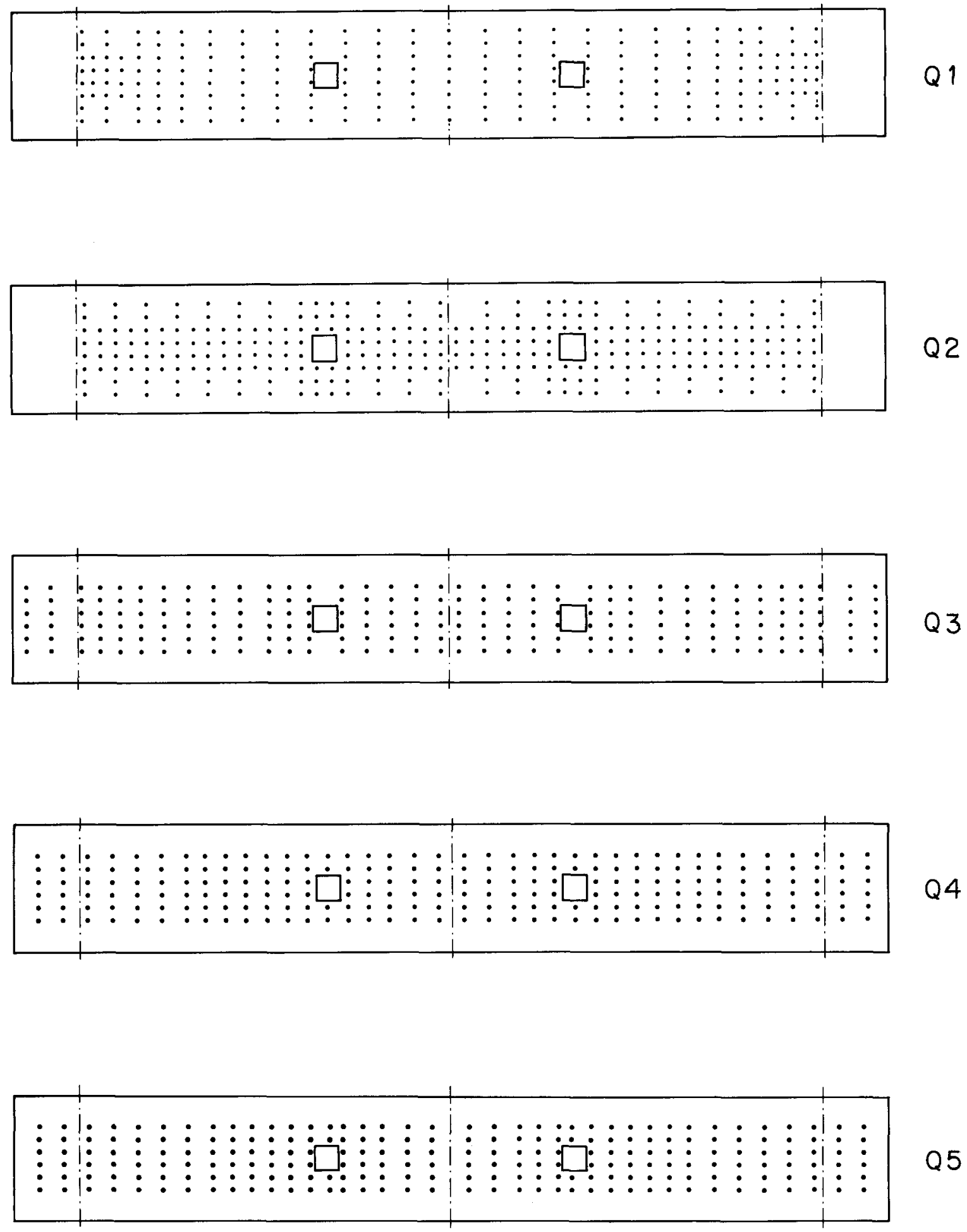

Bild 30: Messstellen an der Plattenarmierung (Oberseite) bei den Balken Q1 $\div$ Q5 


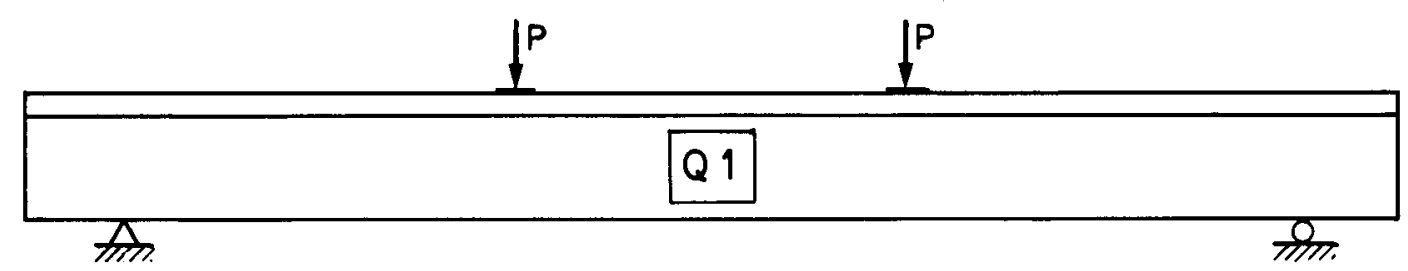

Obere $=$ untere Lage der Plattenarmierung
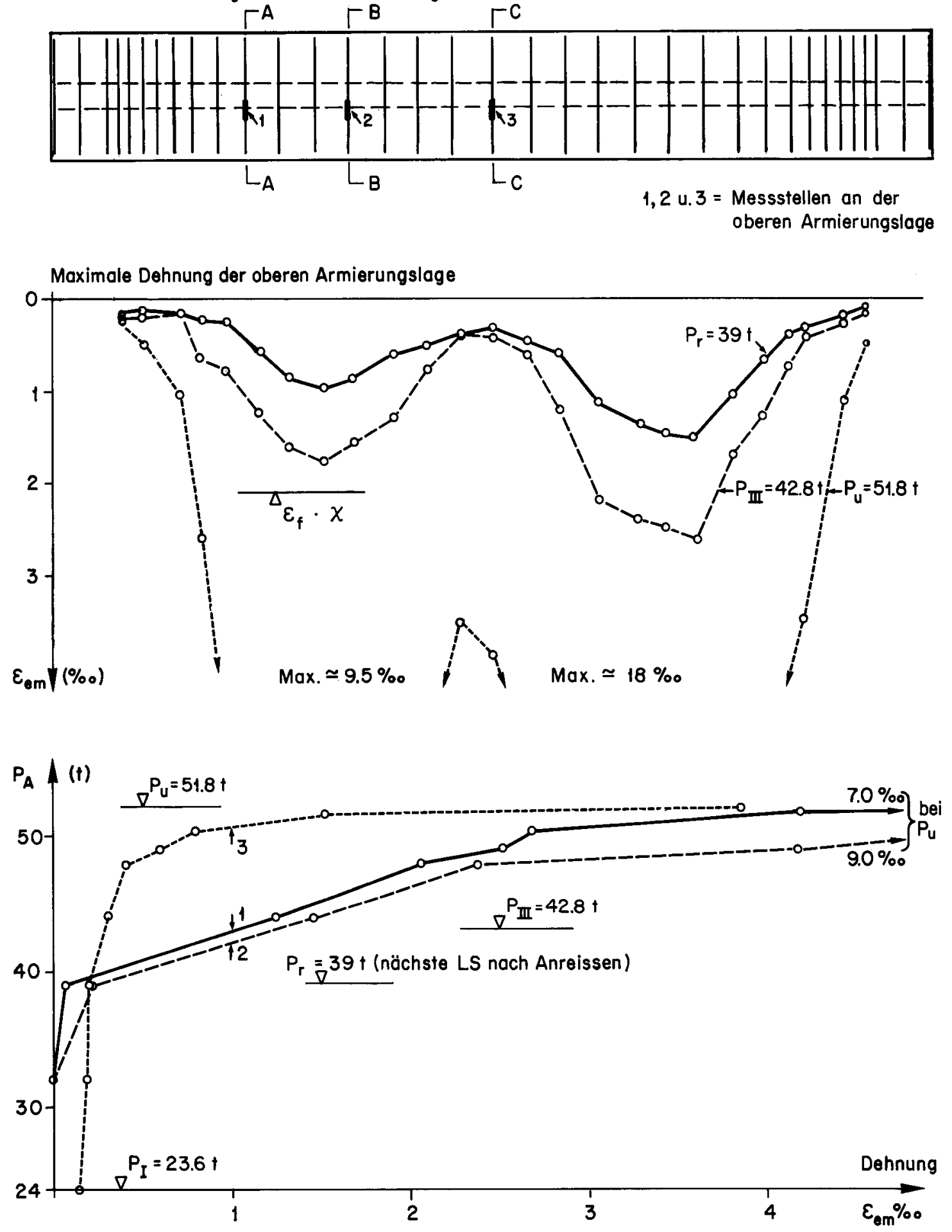

Verlauf der Dehnungen an den Messstellen 1, 2 und 3

Bild 31-a: Dehnung der Plattenarmierung bei Balken Q 1 


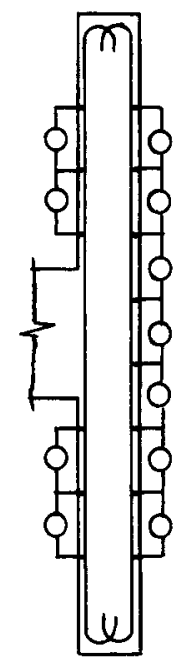

Q 1
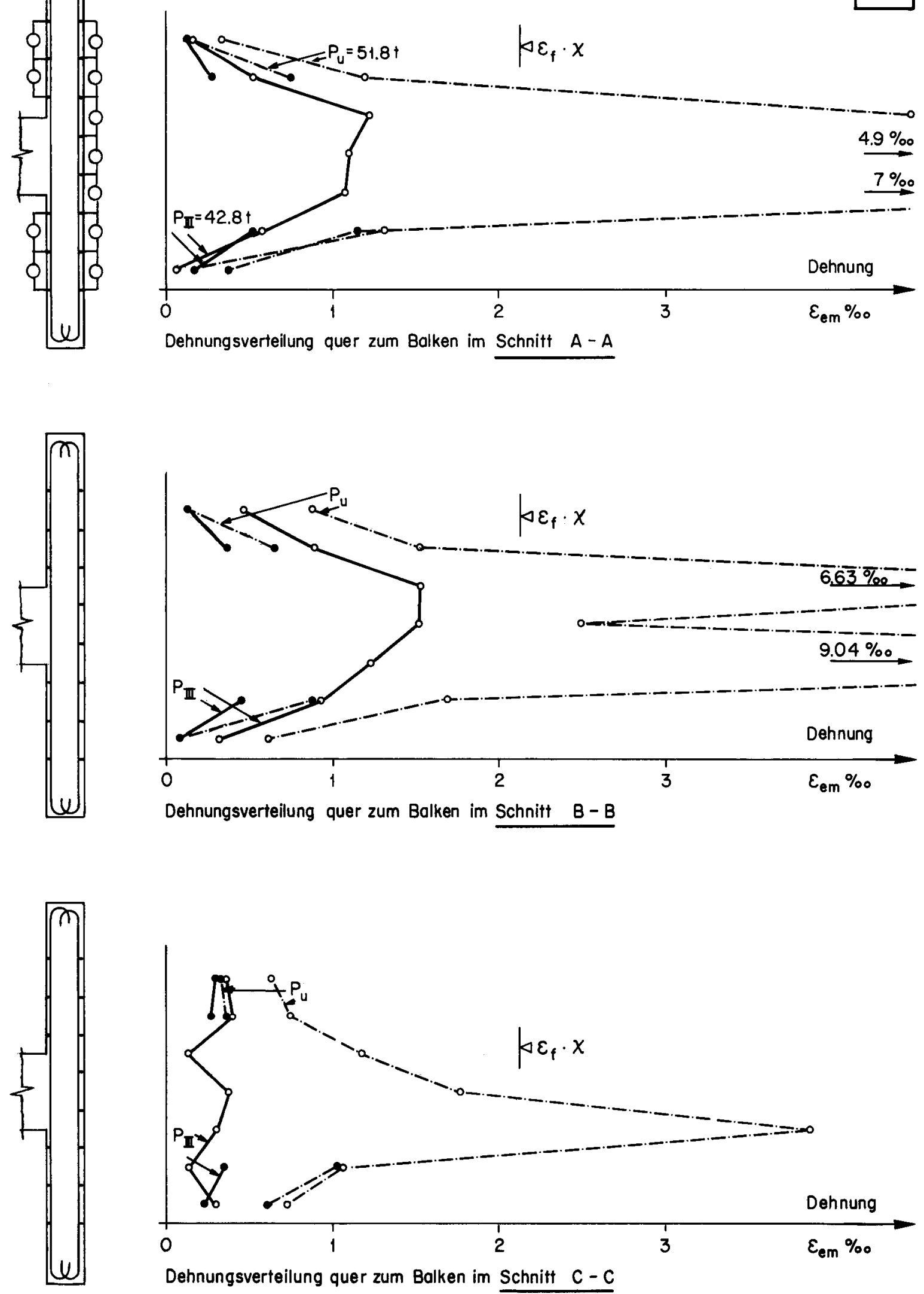

- obere Armierungslage - untere Armierungslage

Bild 31-b: Dehnung der Plattenarmierung bei Balken $Q 1$ 


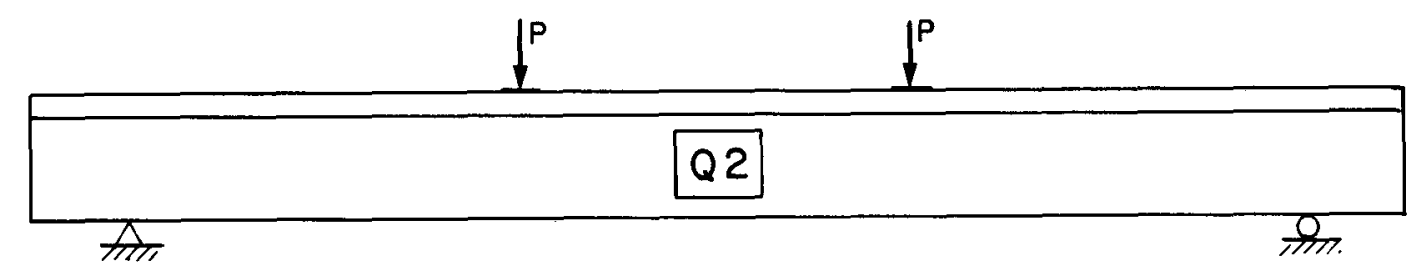

Obere $=$ untere Loge der Plattenarmierung
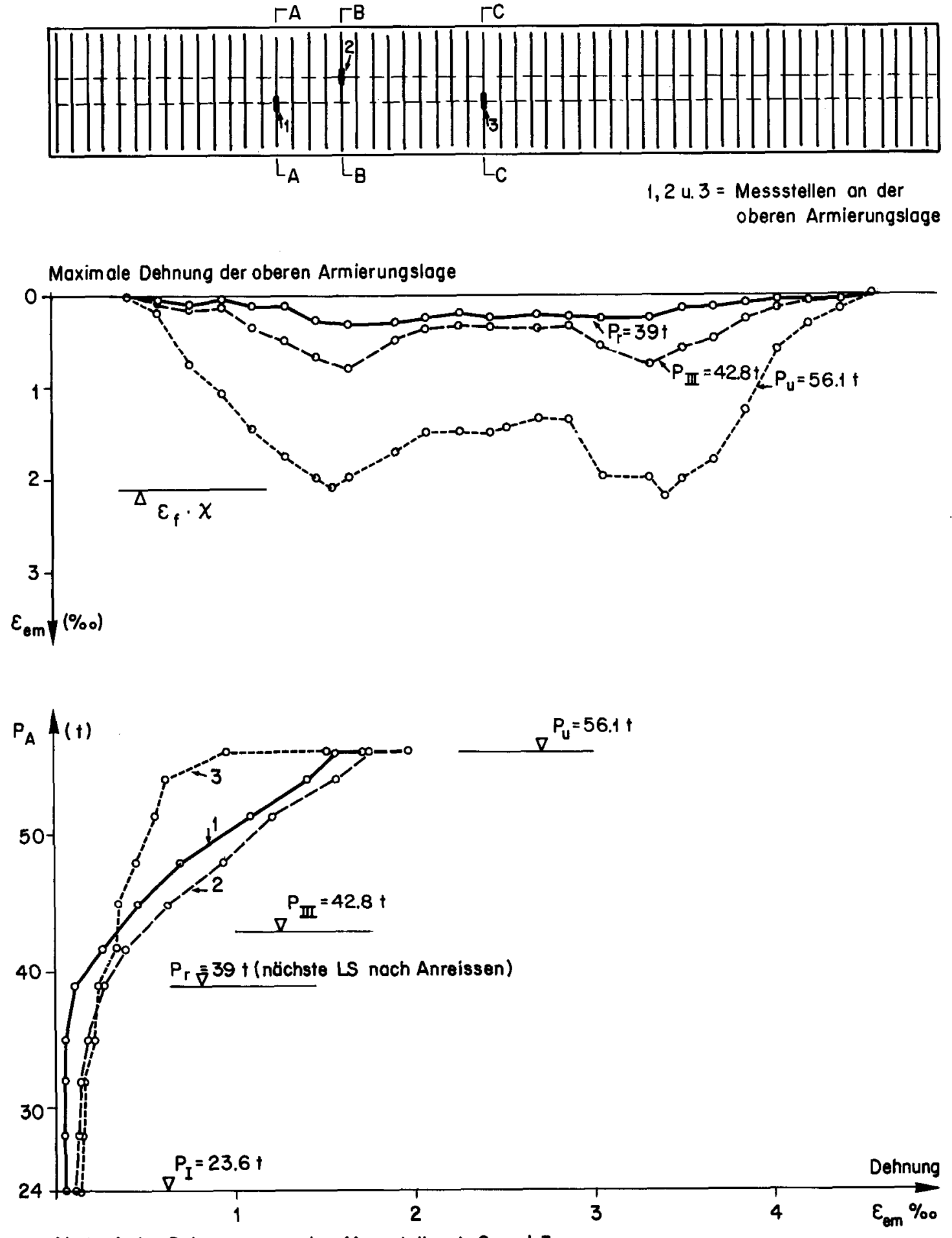

Verlauf der Dehnungen an den Messstellen 4, 2 und 3

Bild 32-a: Dehnung der Plattenarmierung bei Balken Q 2 

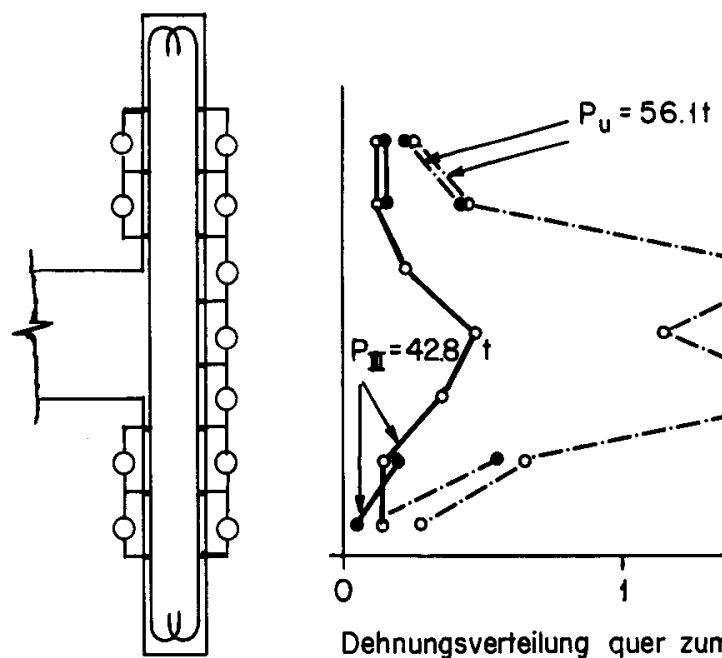
$\Delta \varepsilon_{f} \cdot x$
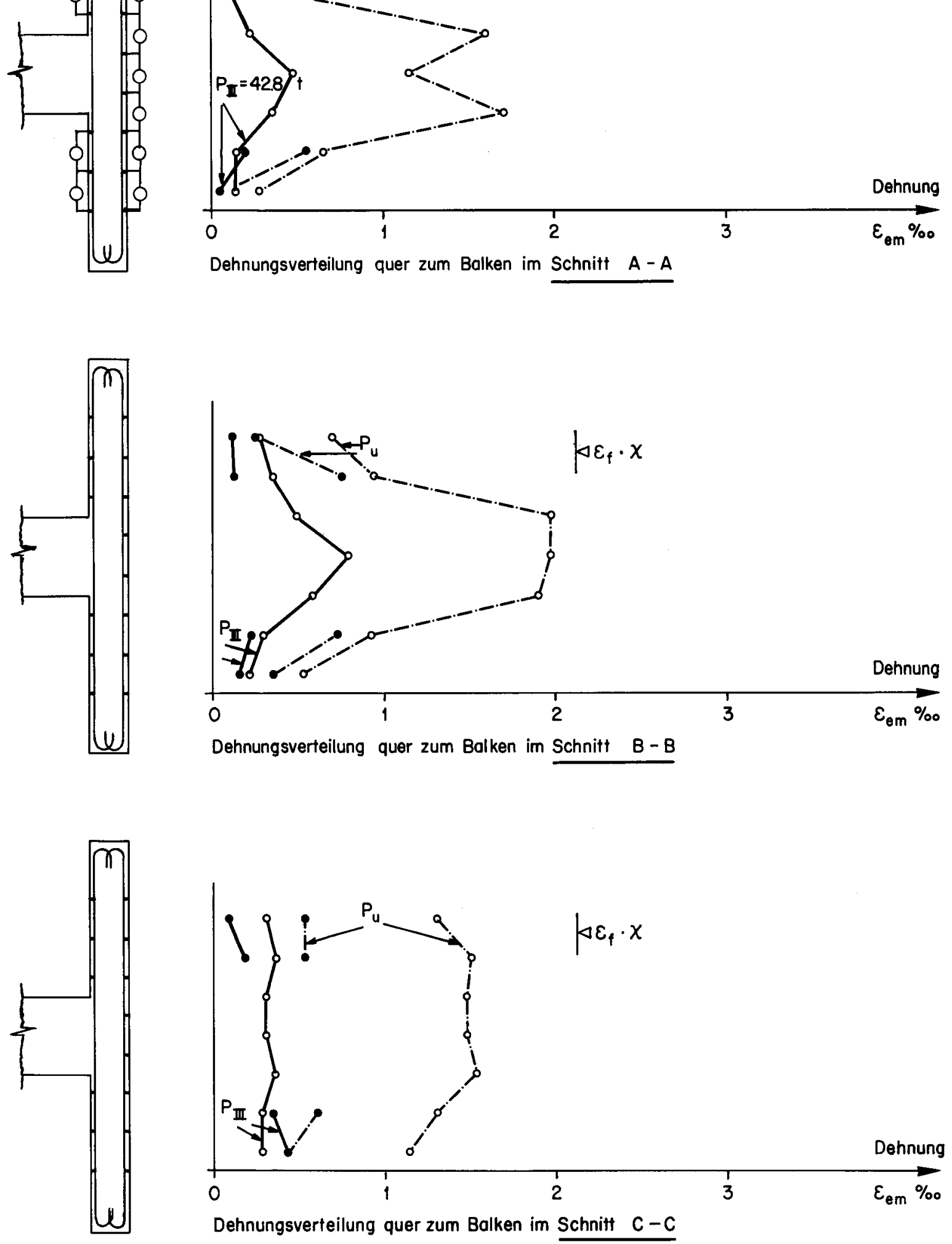

Bild 32-b: Dehnung der Plattenarmierung bei Balken $Q 2$ 


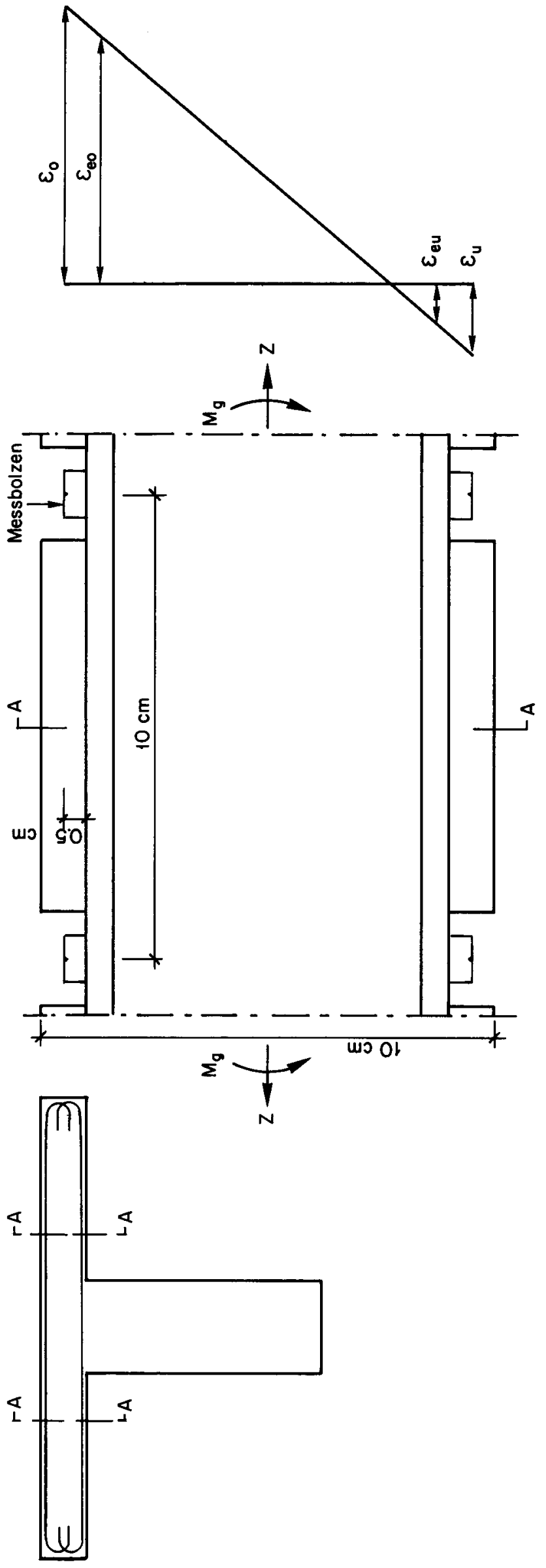

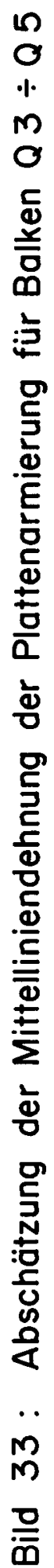



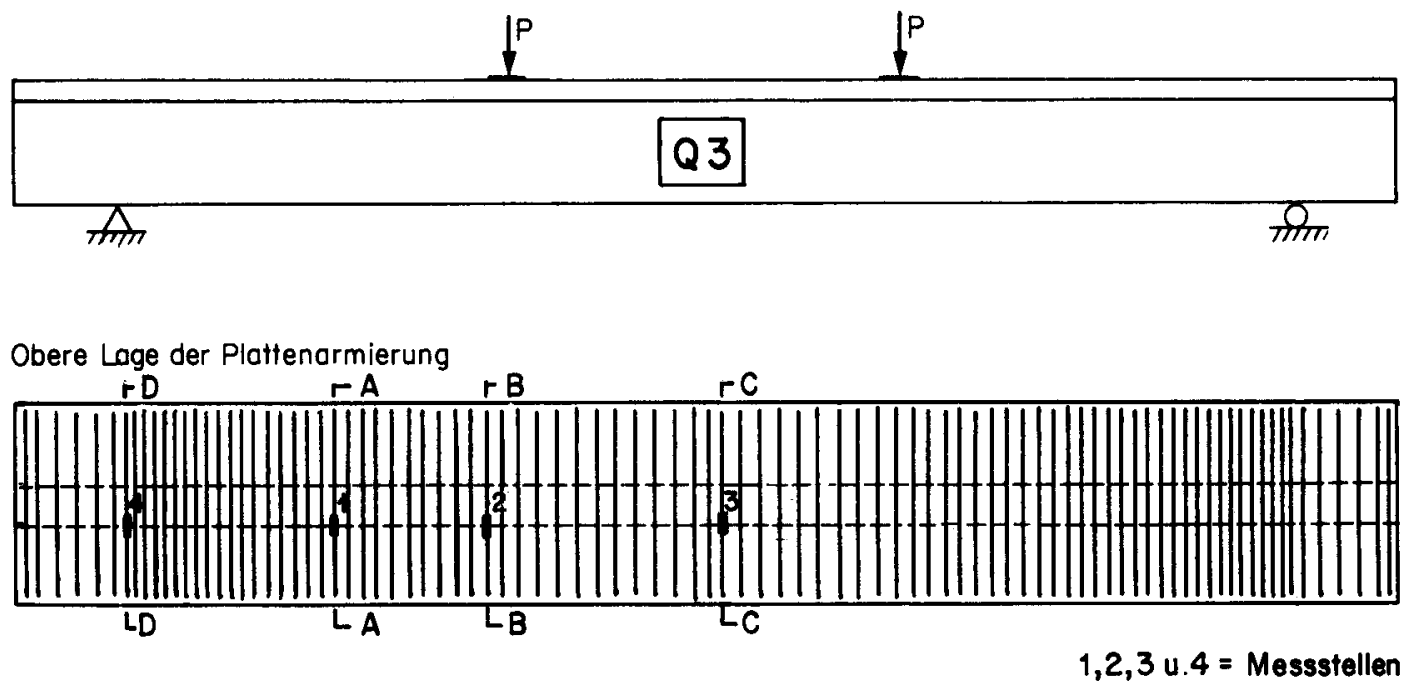

Untere Lage der Plattenarmierung
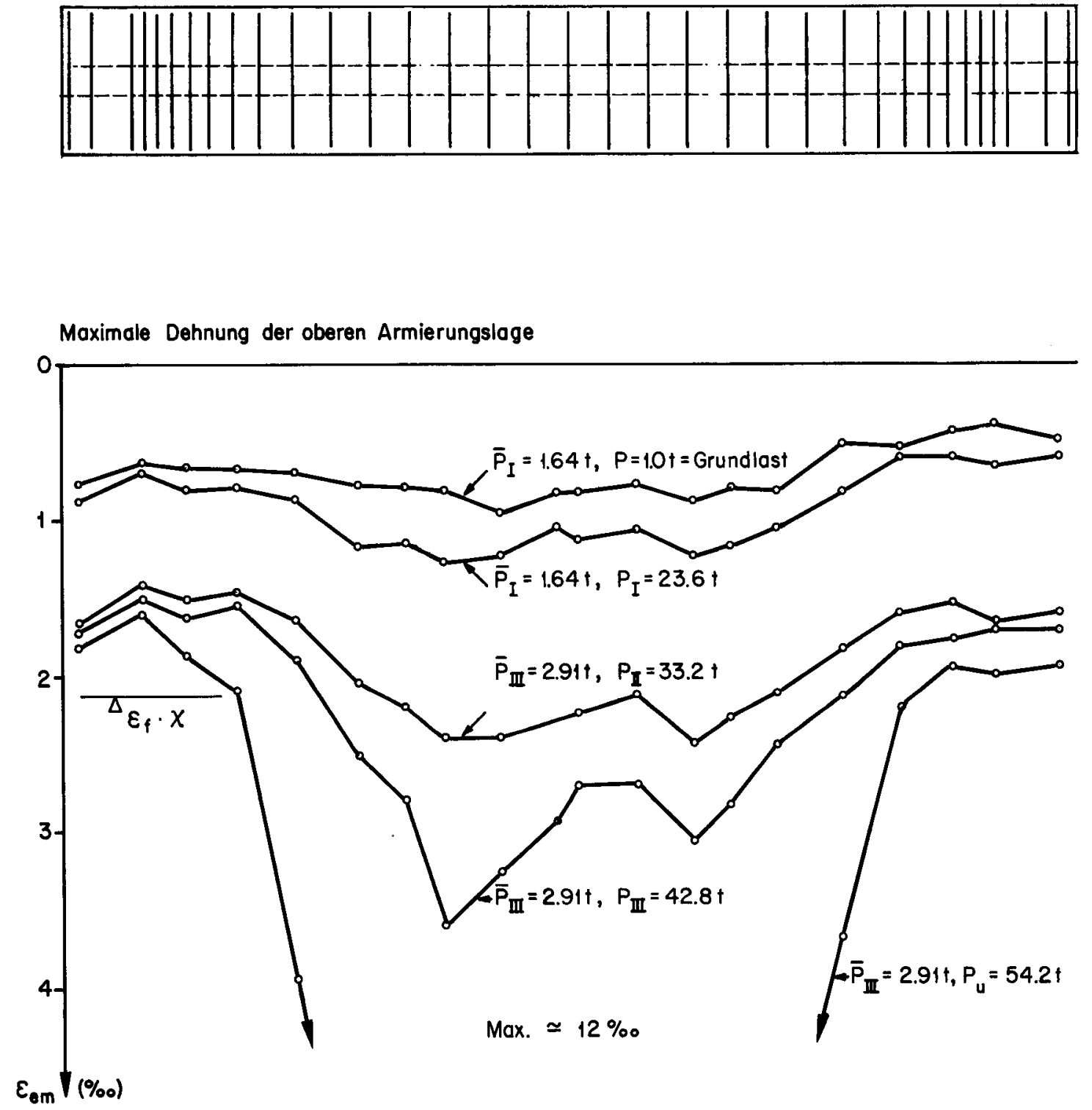

Bild 34-a: Dehnung der Plattenarmierung bei Balken Q 3 

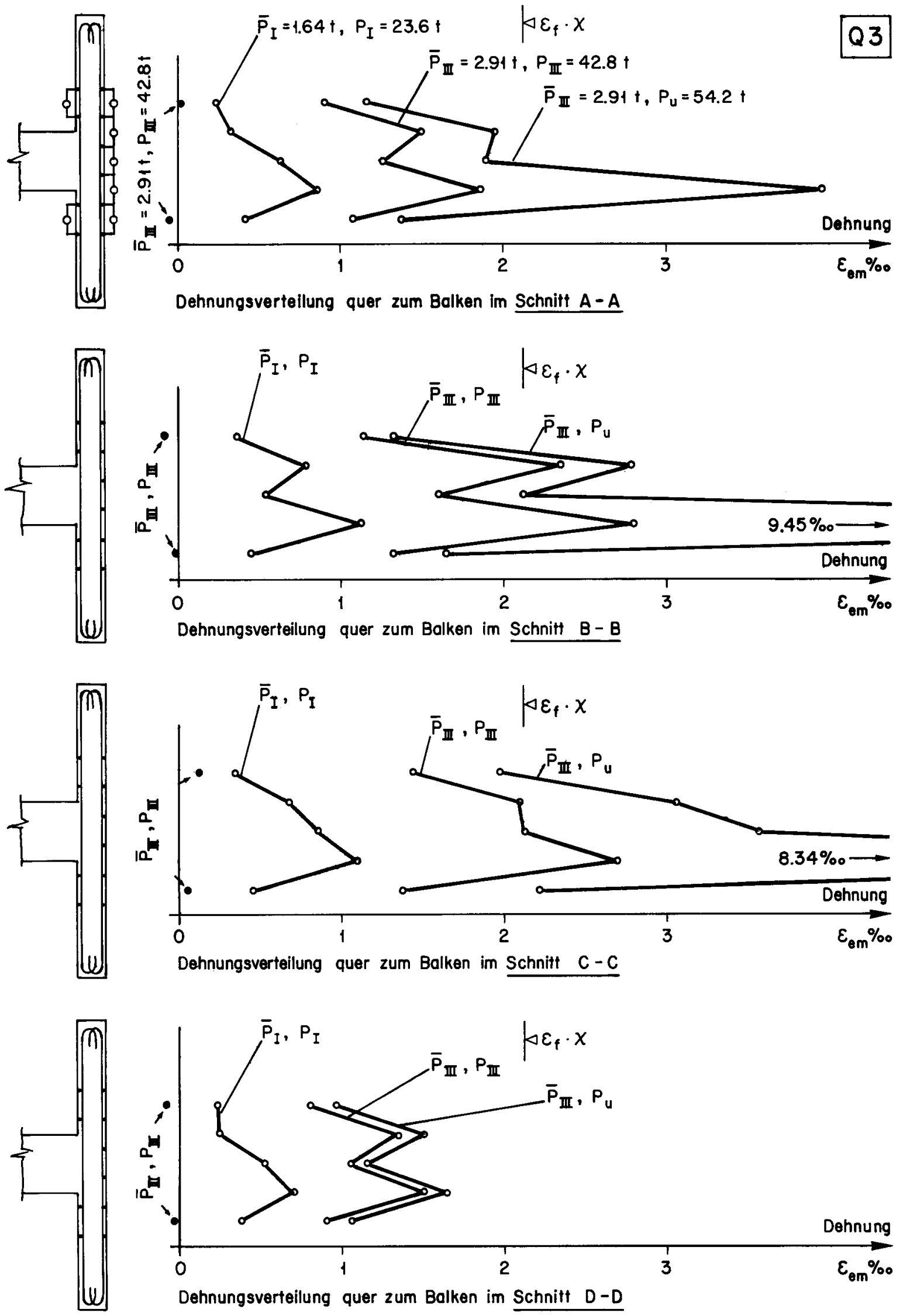

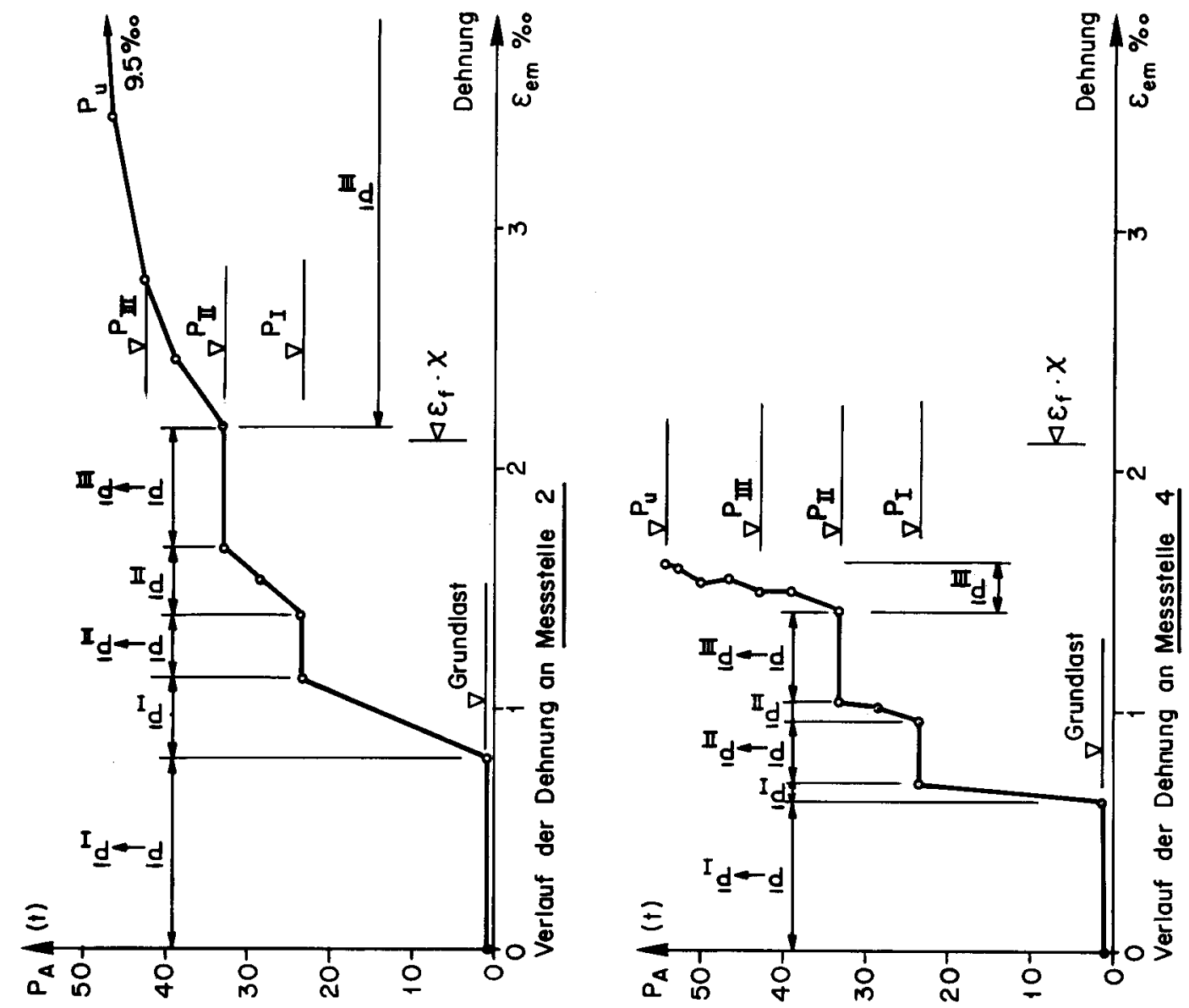

m
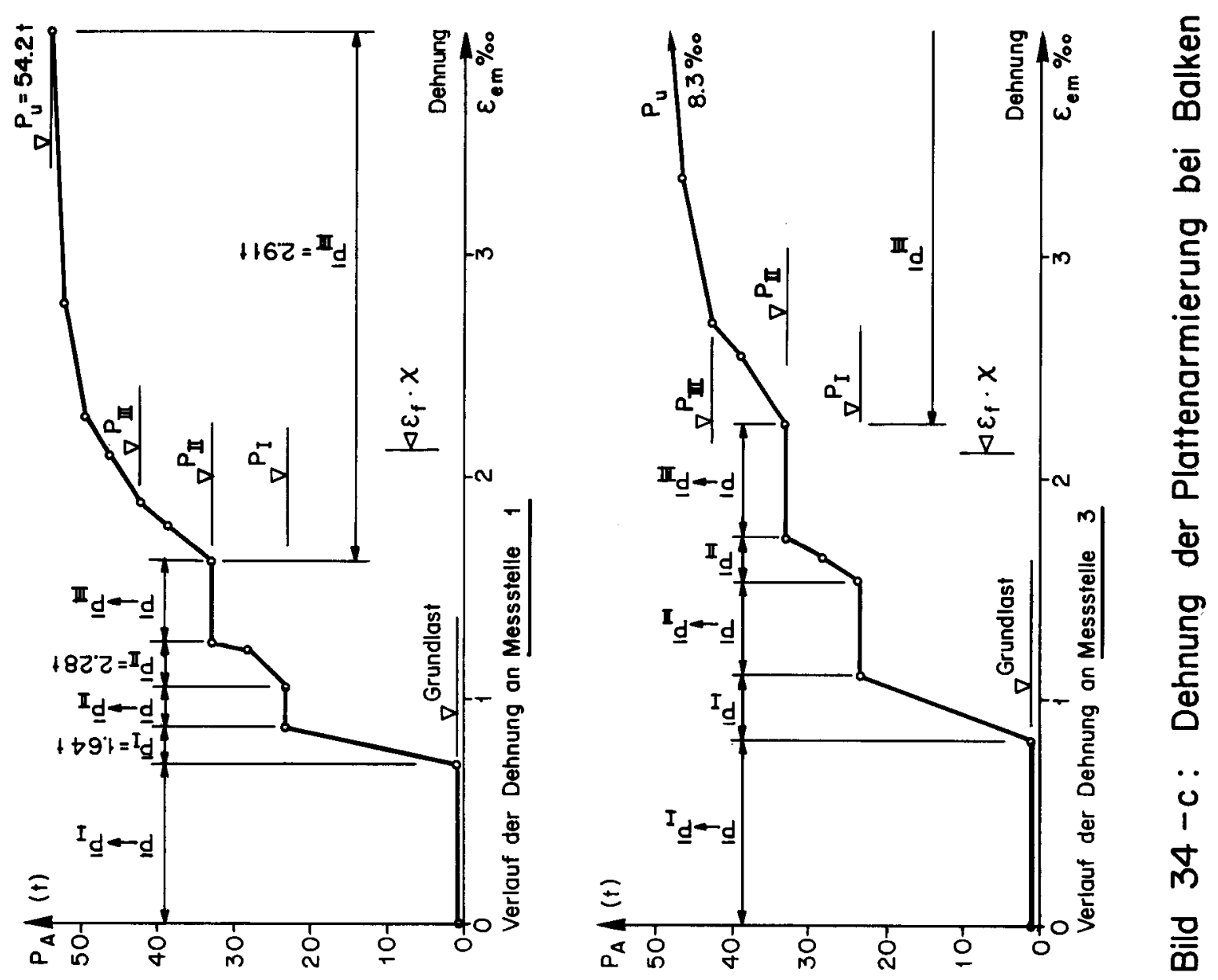

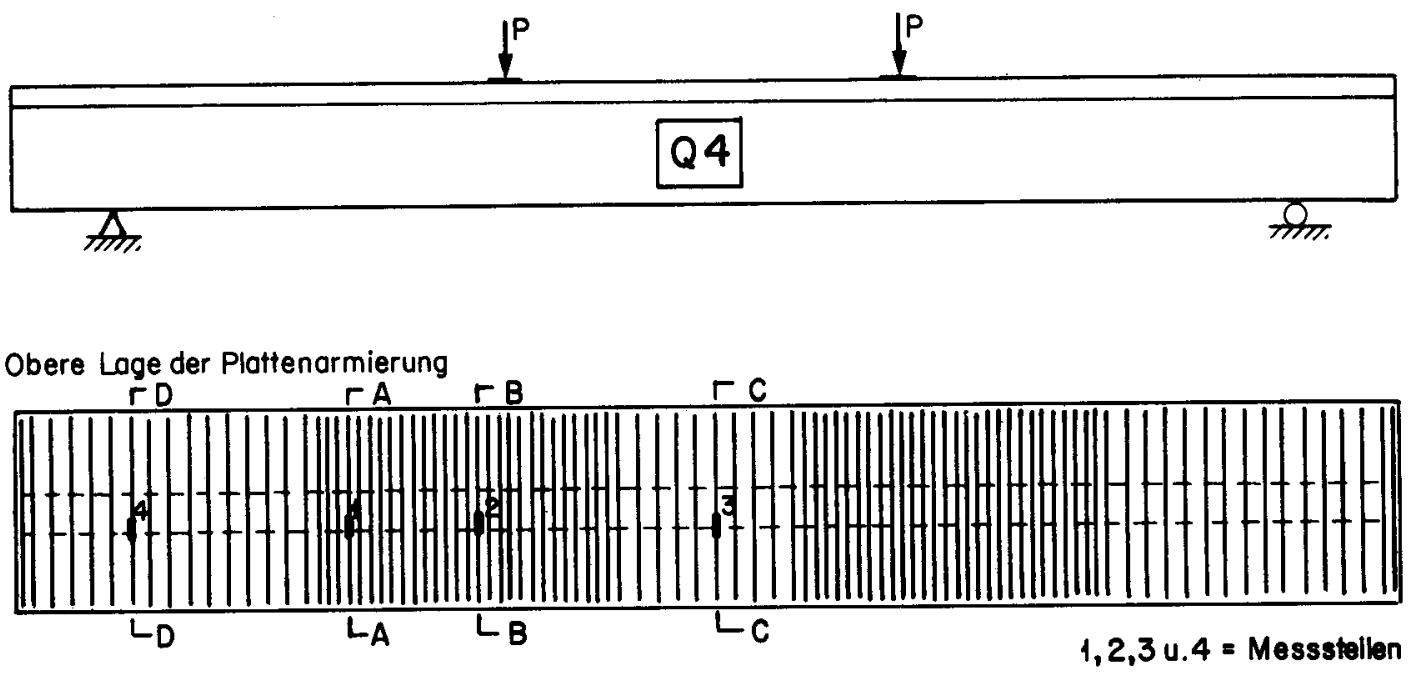

Untere Lage der Plattenarmierung

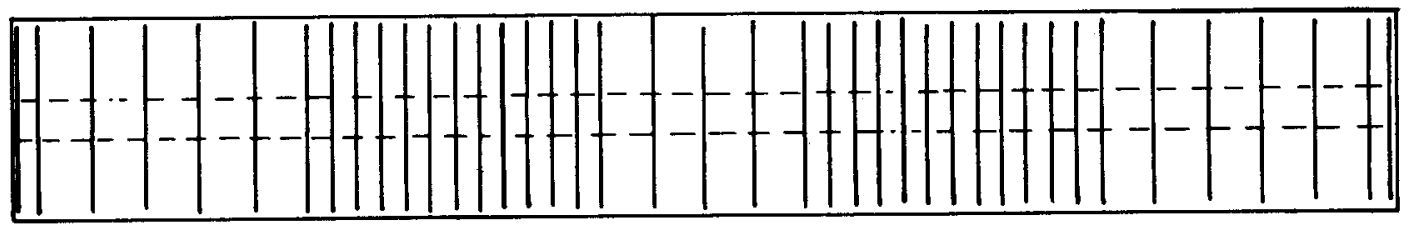

Maximale Dehnung der oberen Armierungslage

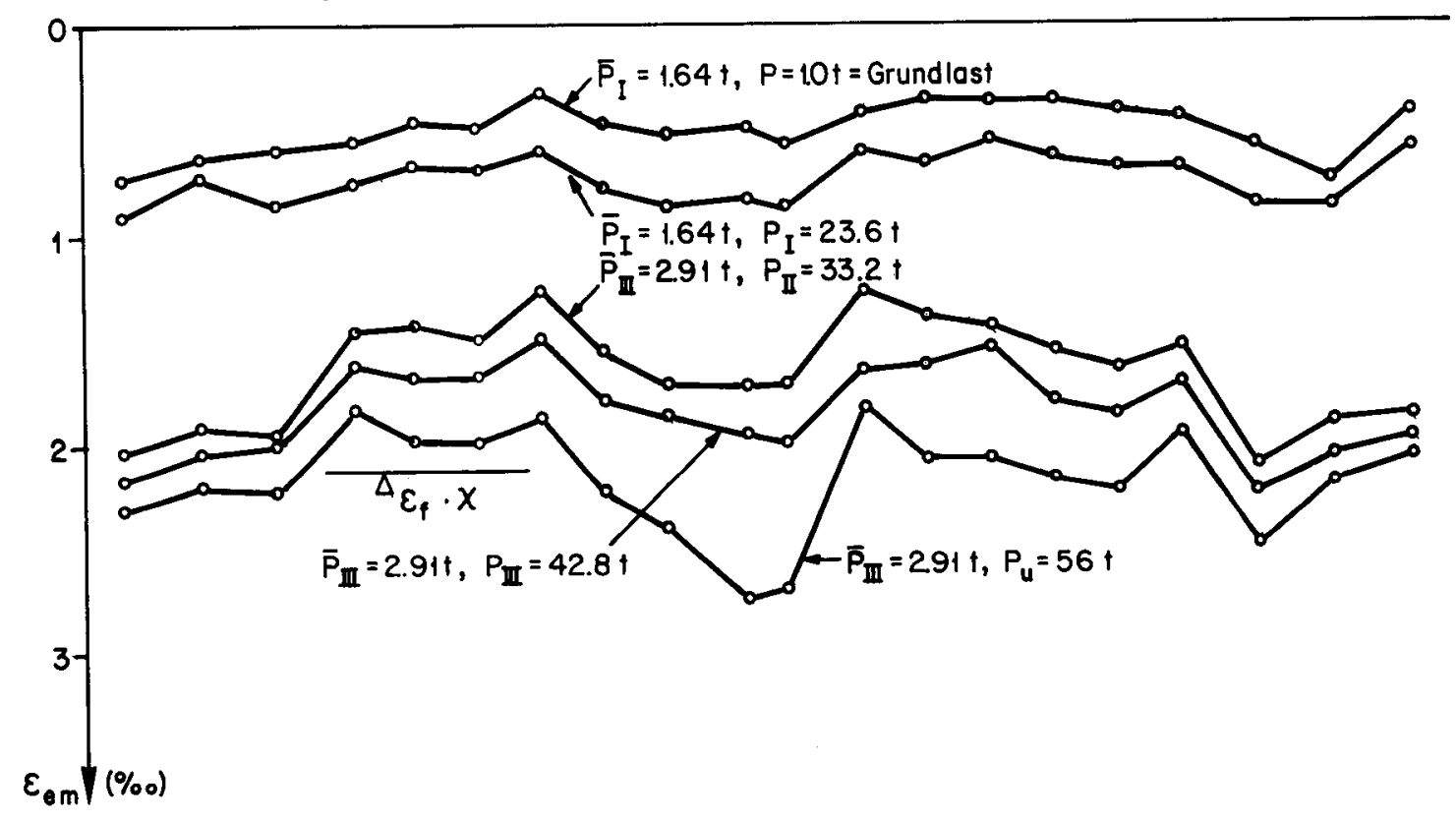

Bild 35-a: Dehnung der Plattenarmierung bei Balken $Q 4$ 

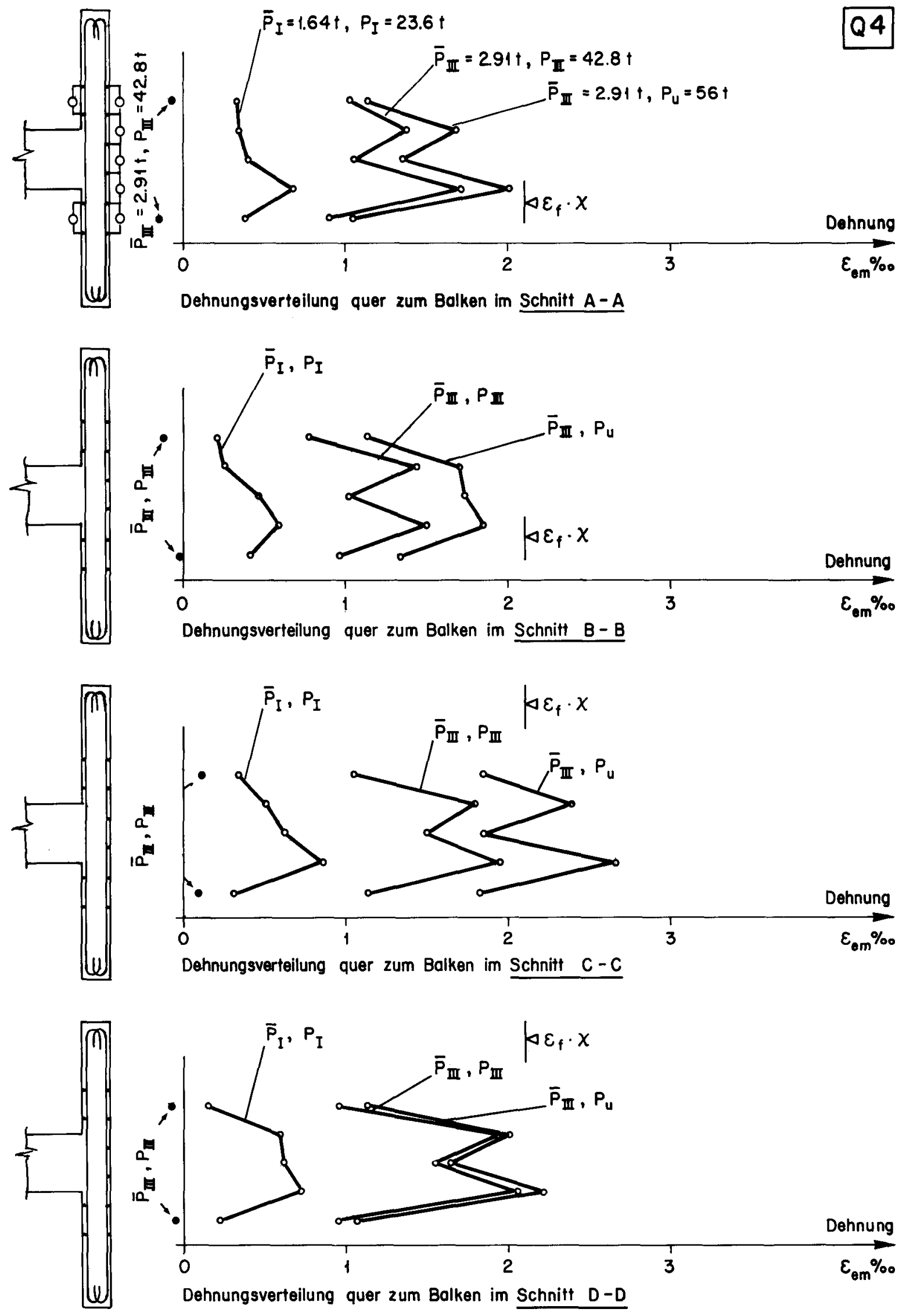

Bild 35-b: Dehnung der Plattenarmierung bei Balken Q 4 

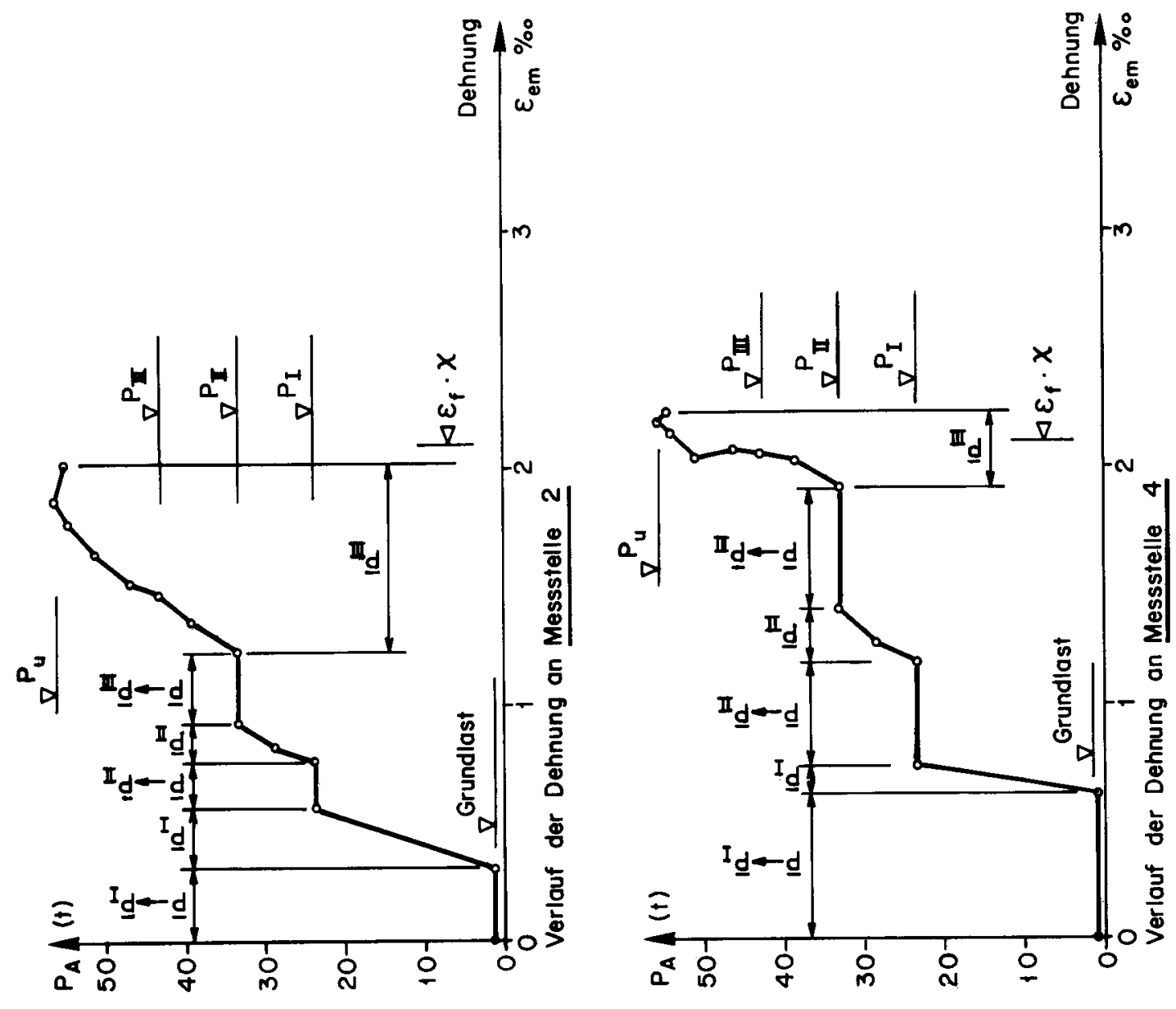

+
0

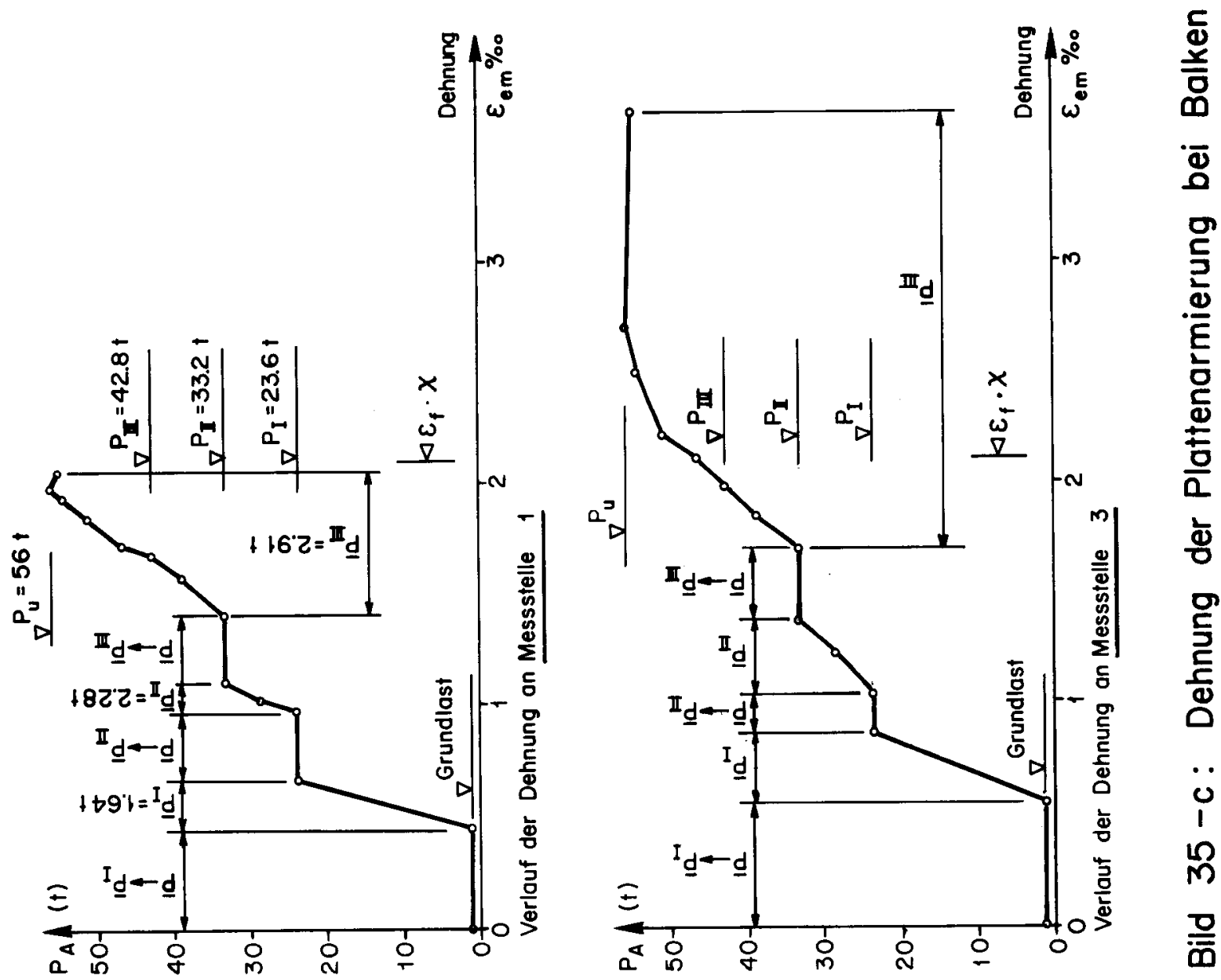



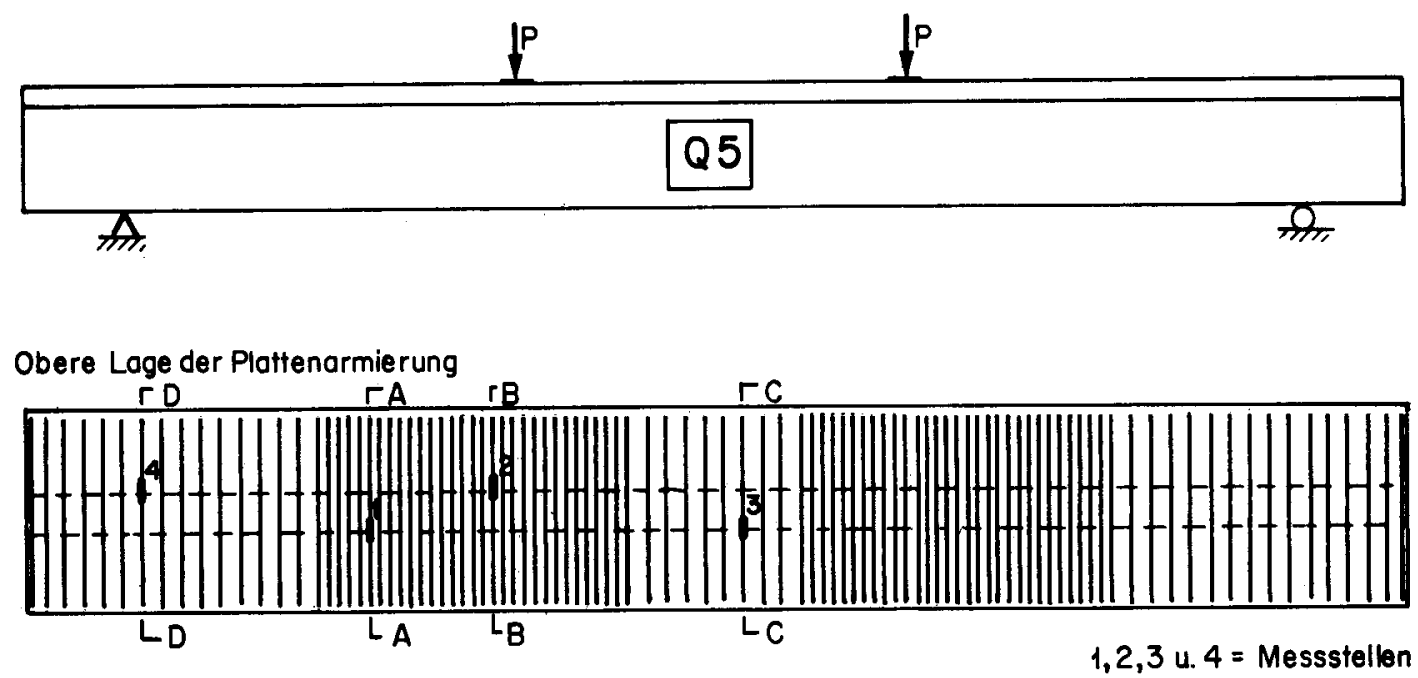

Untere Lage der Plattenarmierung
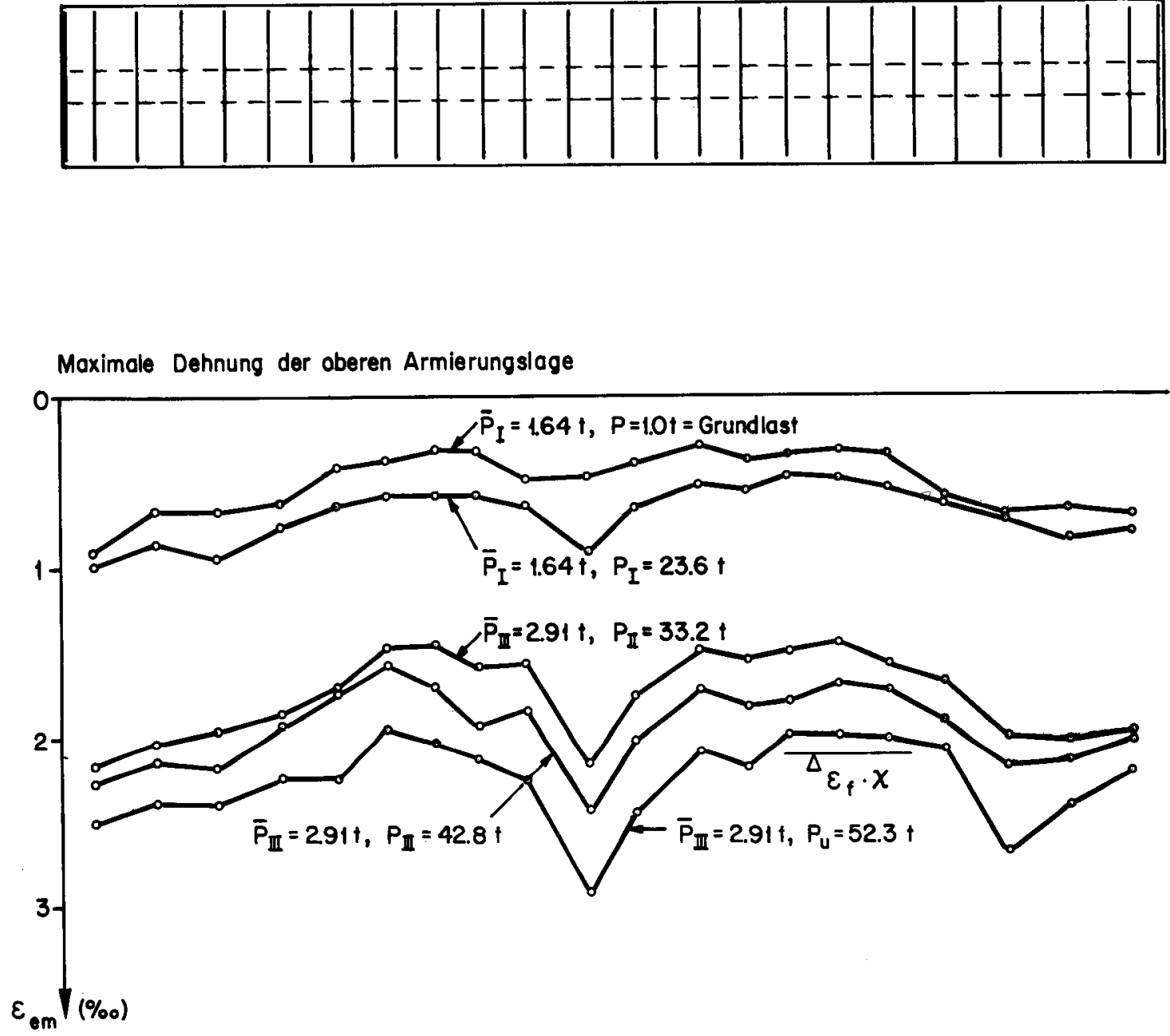

Bild 36-a: Dehnung der Plattenarmierung bei Balken Q 5 

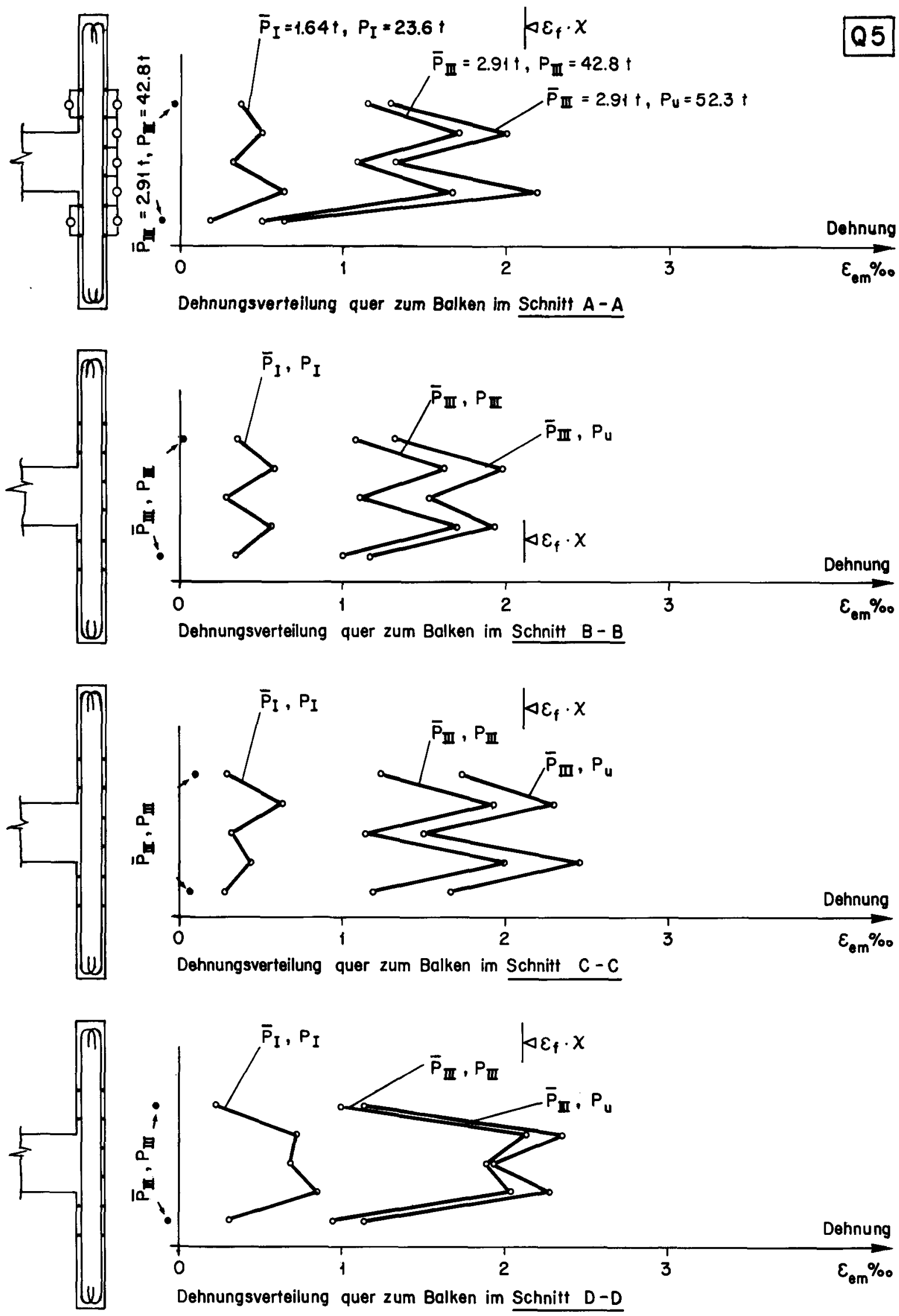

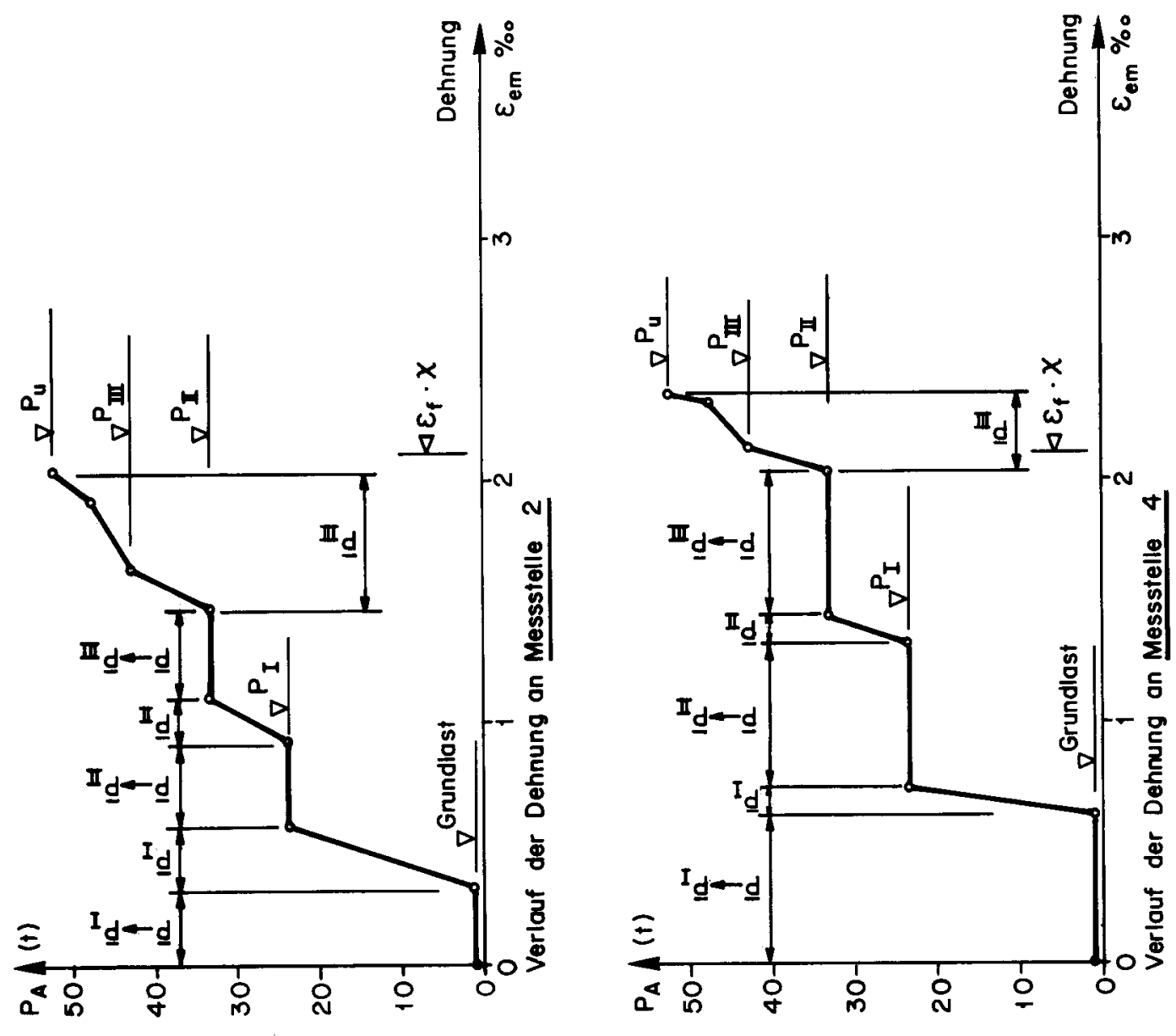

0
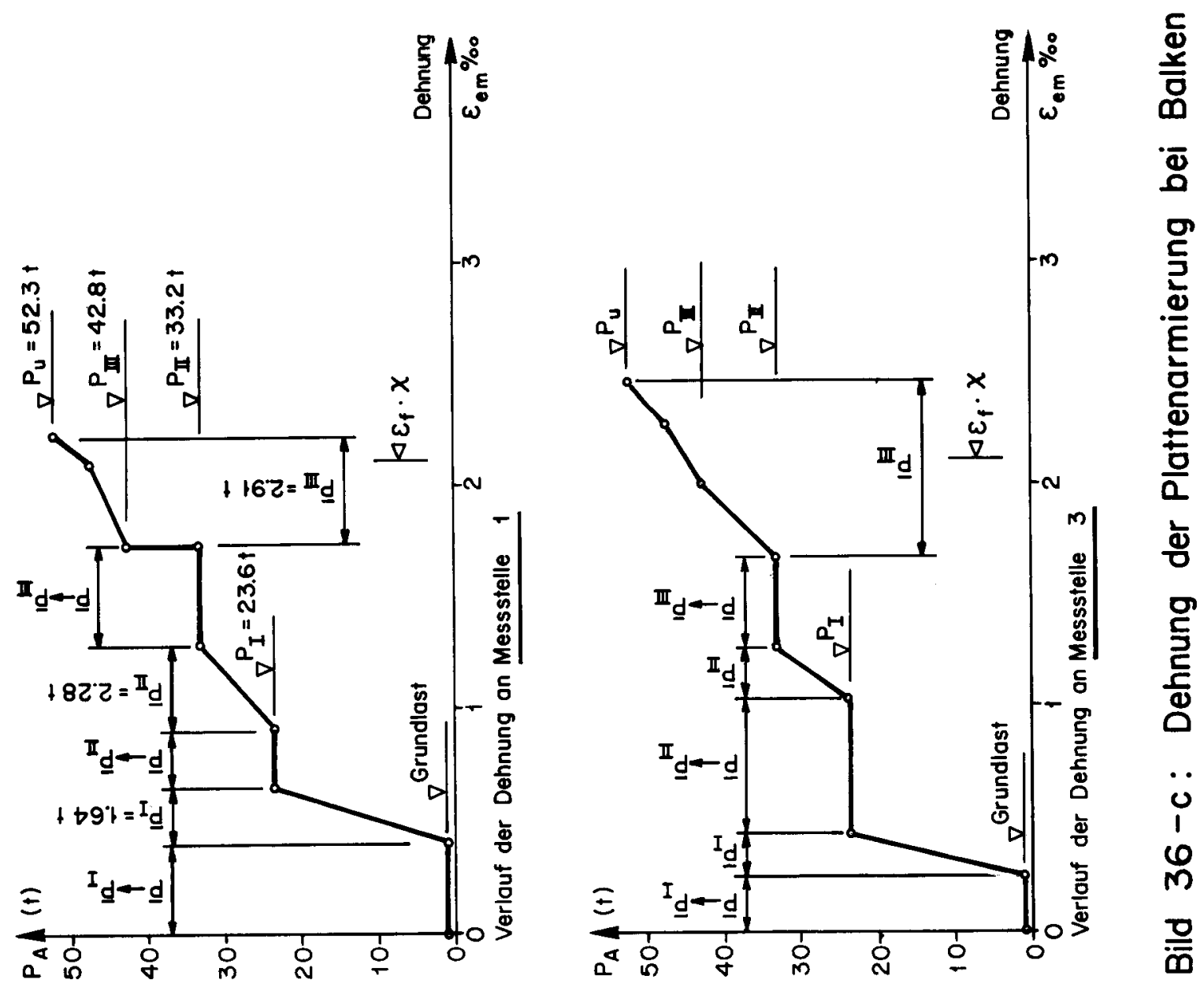


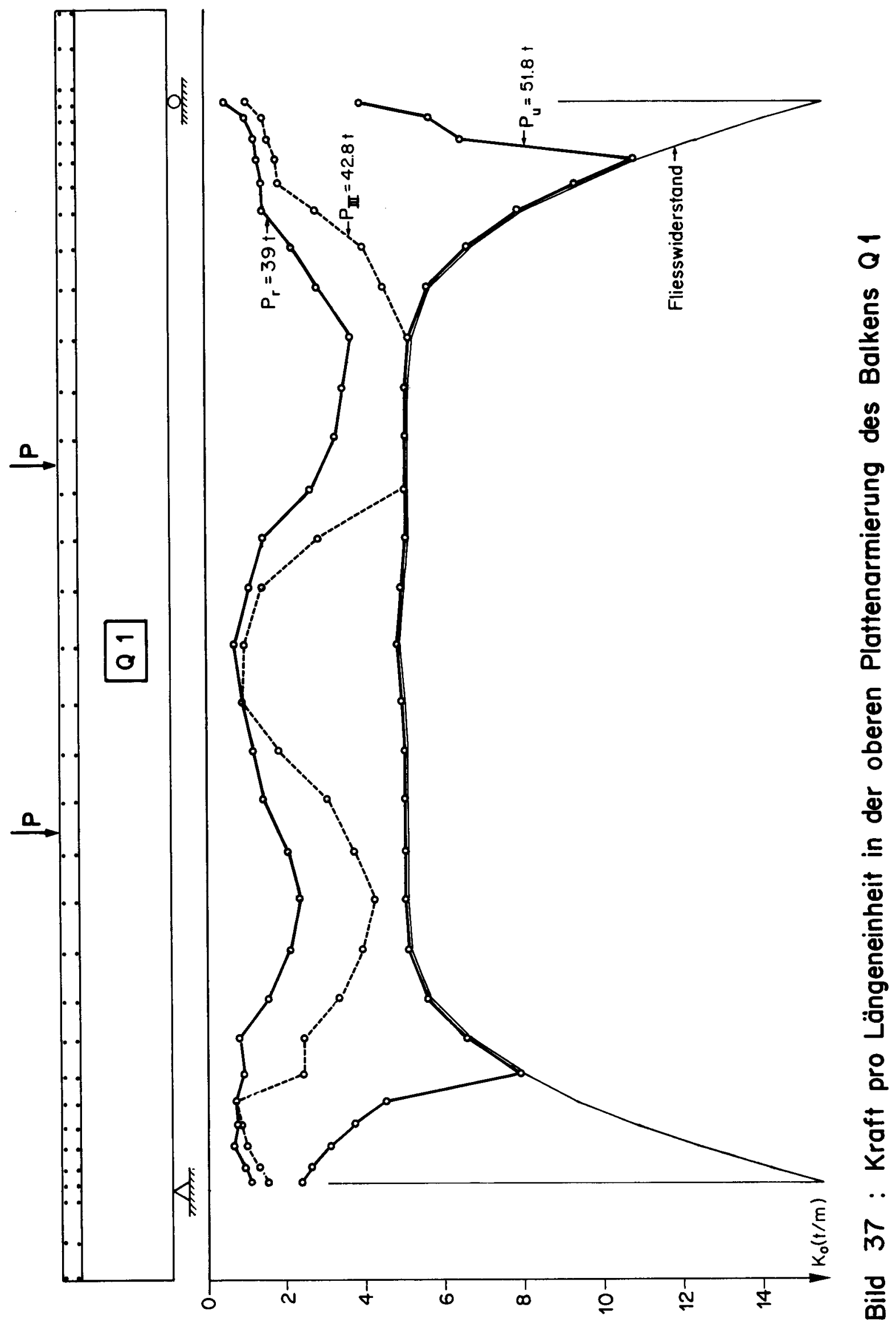




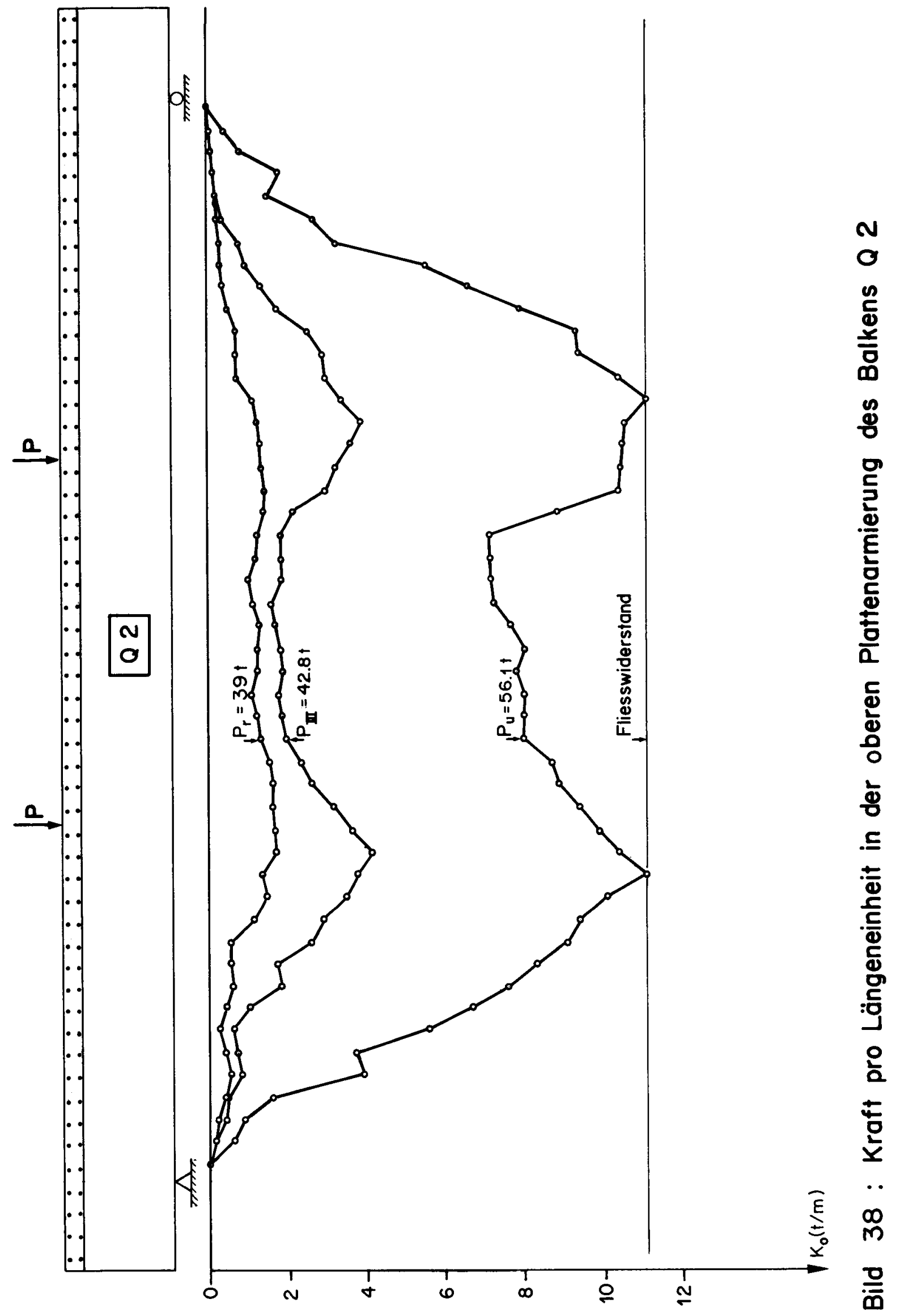




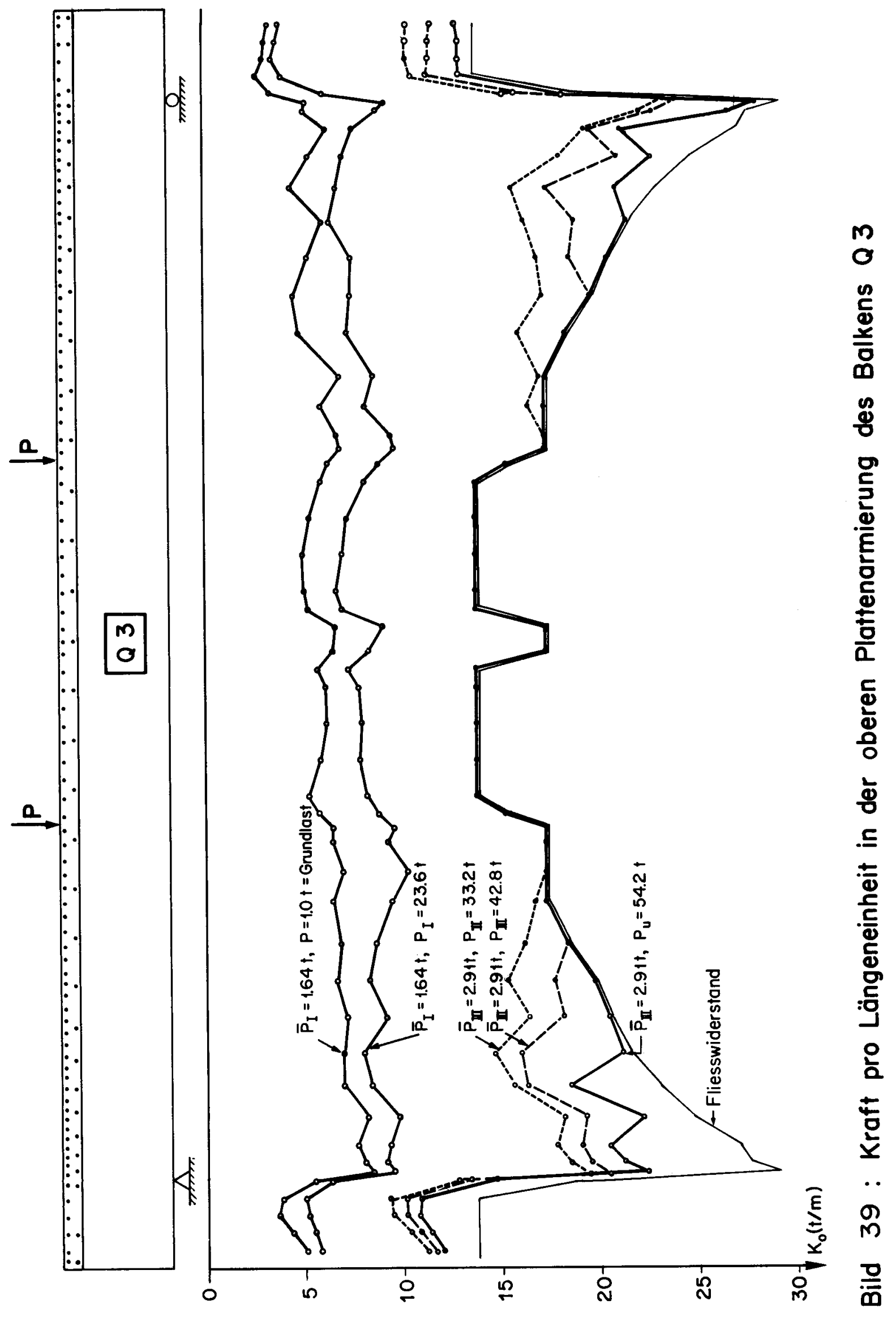




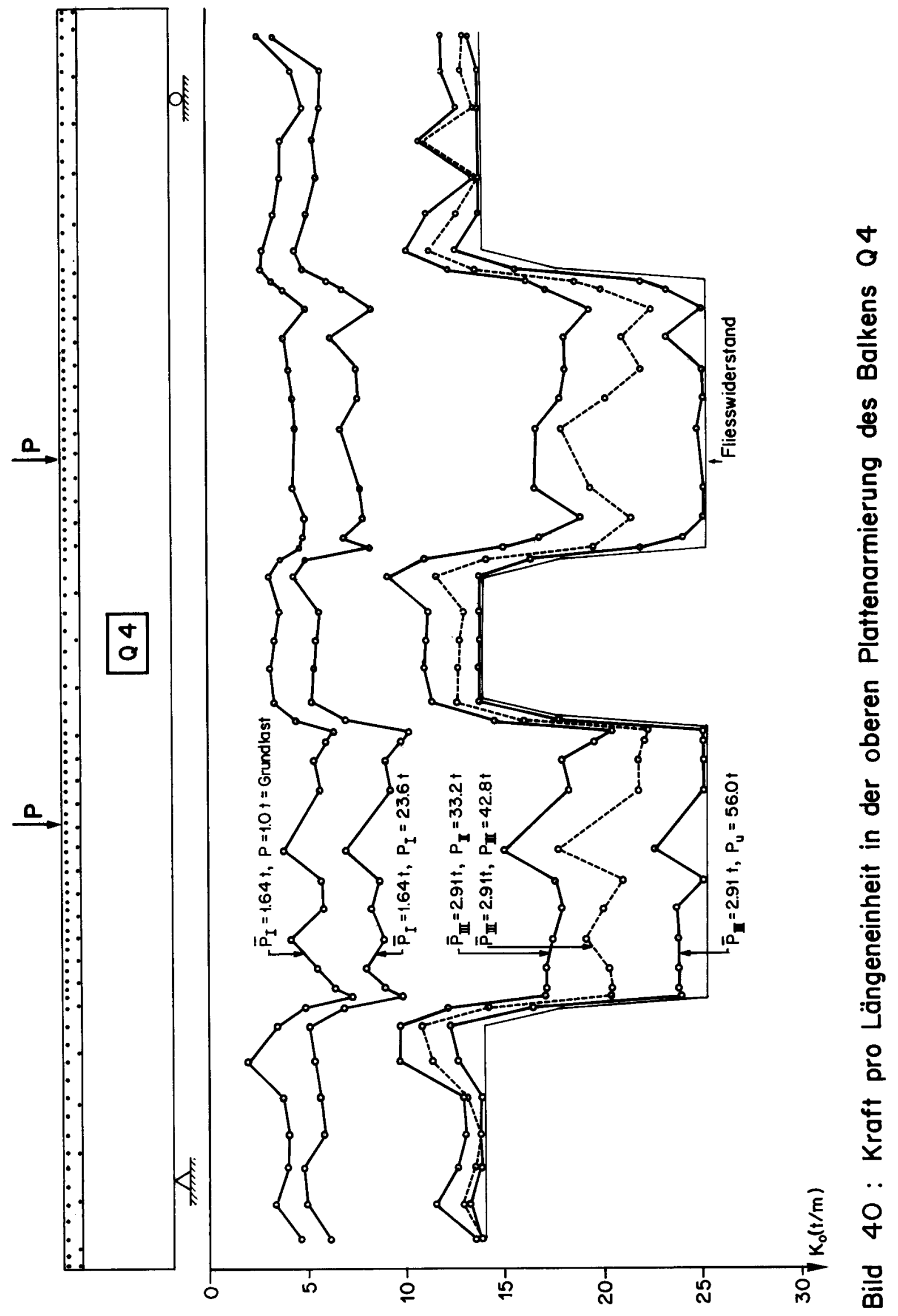




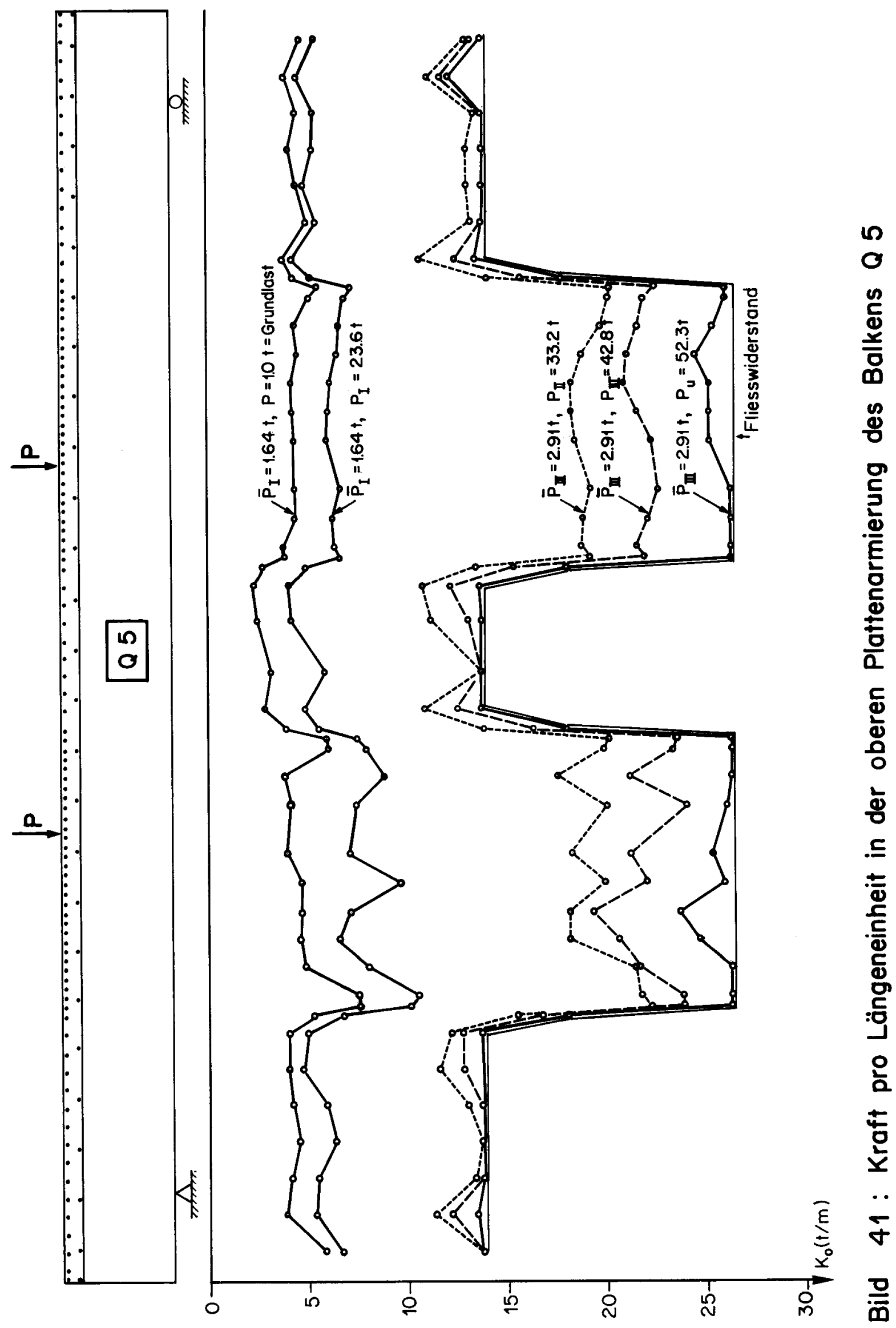




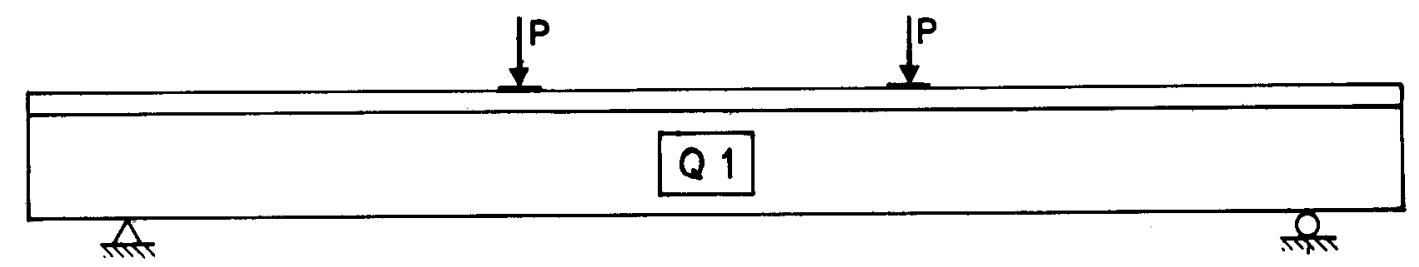

obere = untere Lage der Plattenarmierung

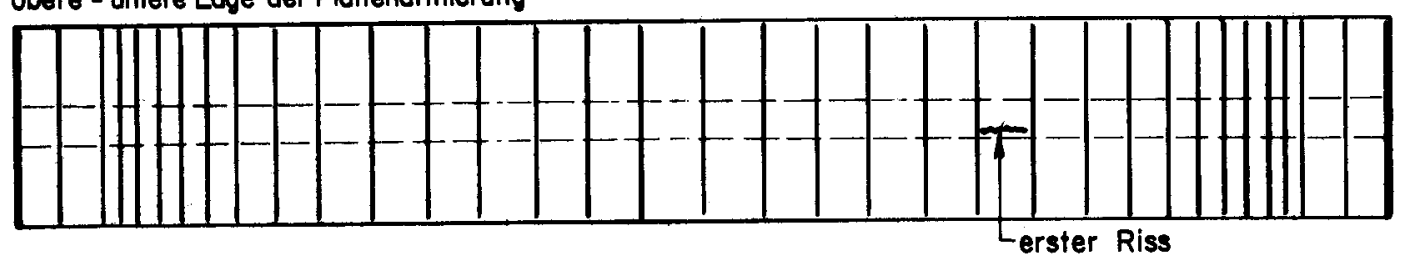

Maximale Rissbreite längs der Platte
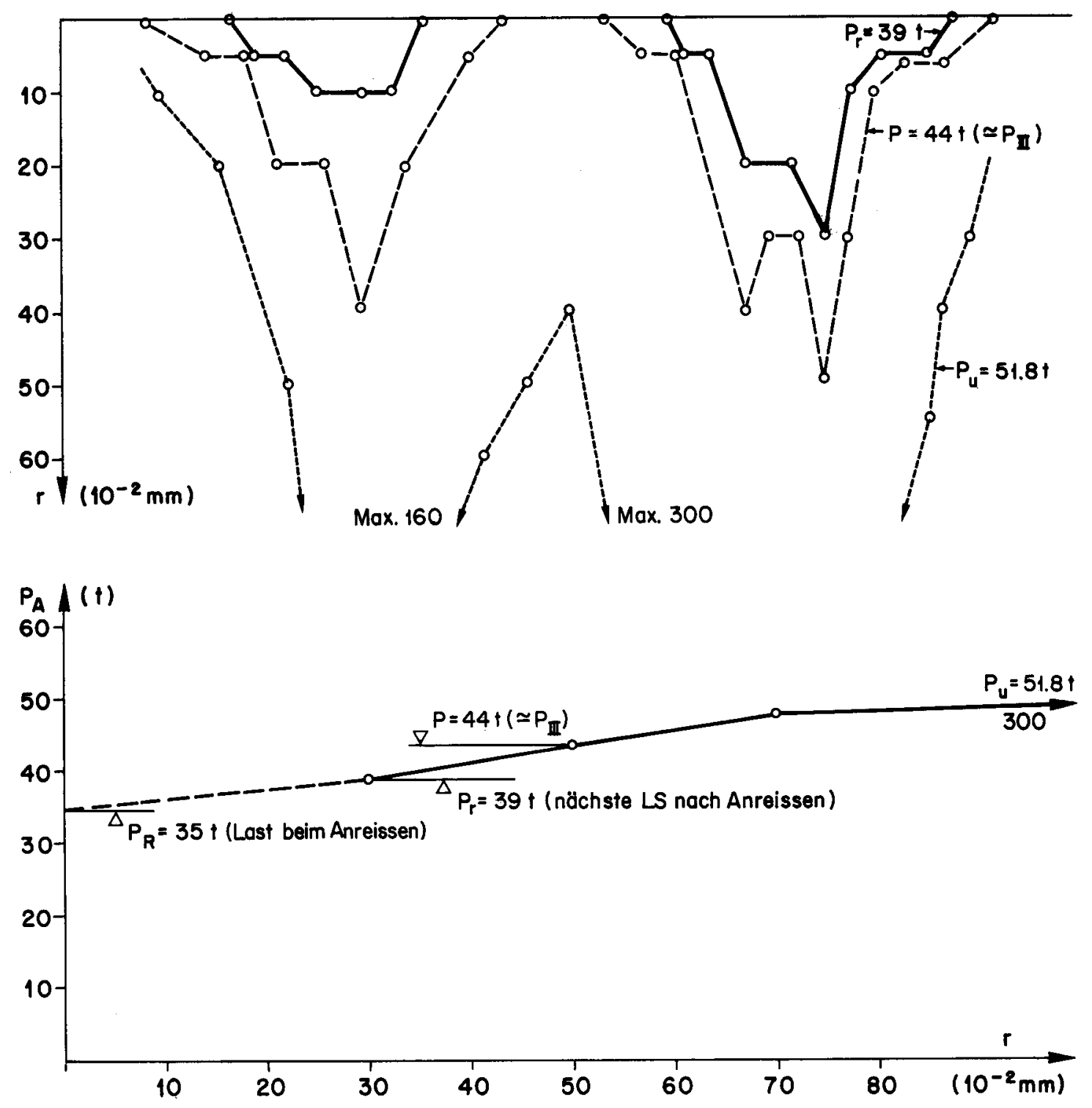

Maximale Rissbreite in Abhängigkeit der Lasł

Bild 42: Rissbreiten auf der Plattenoberseite bei Balken Q 1 


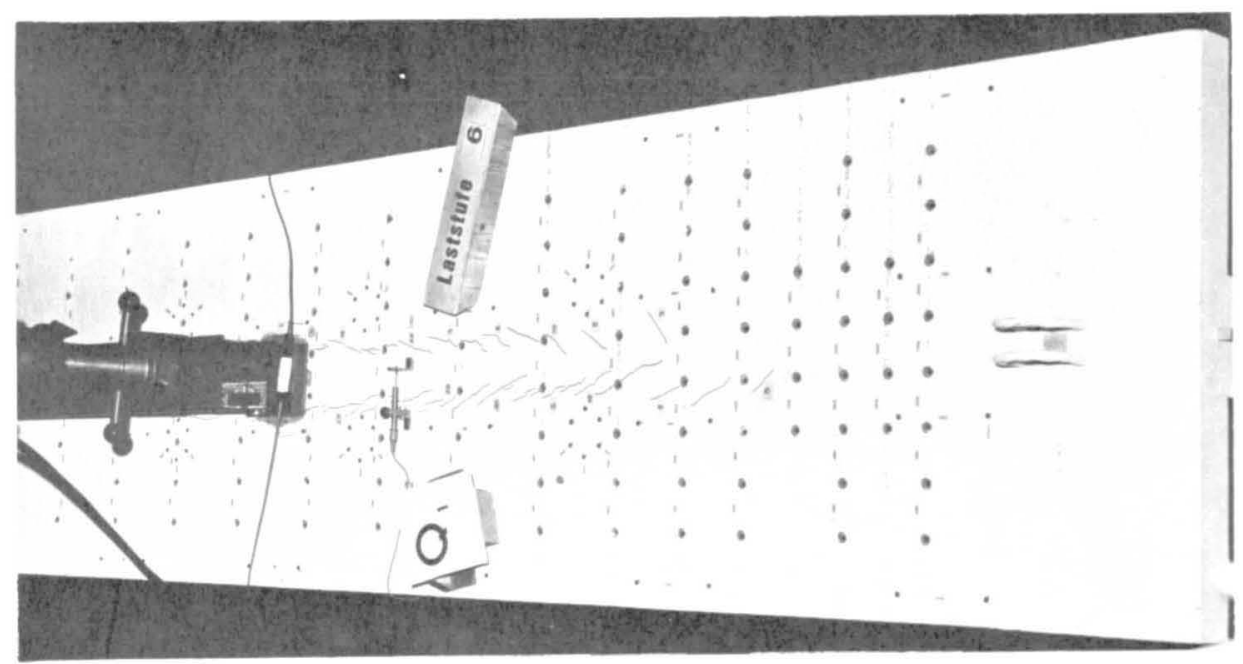

$P_{r}=39+$

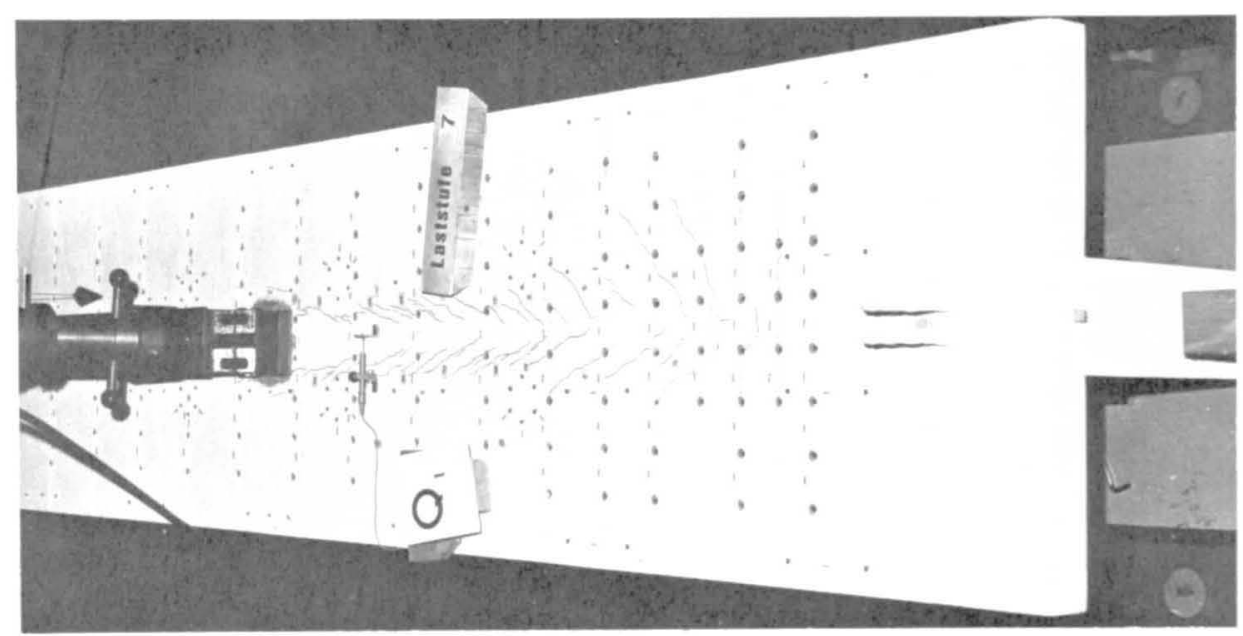

$\mathrm{P}=44 \dagger \approx \mathrm{P}_{\text {III }}$

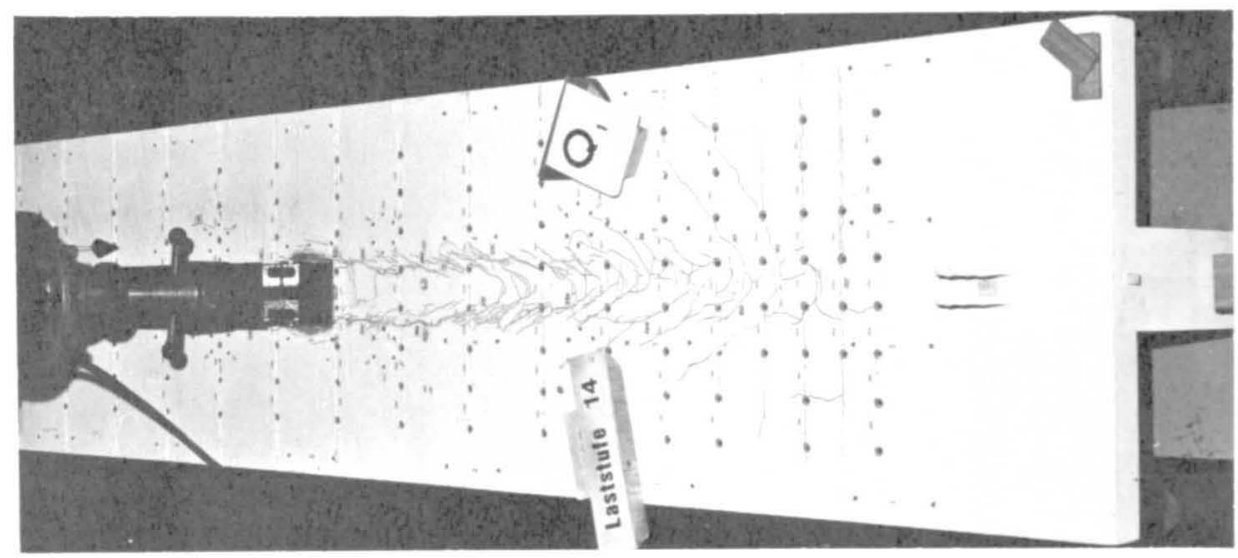

$P_{u}=51.8 \uparrow$

Bild 43: Rissbilder der Platte bei Balken Q1 (rechter Bereich). 


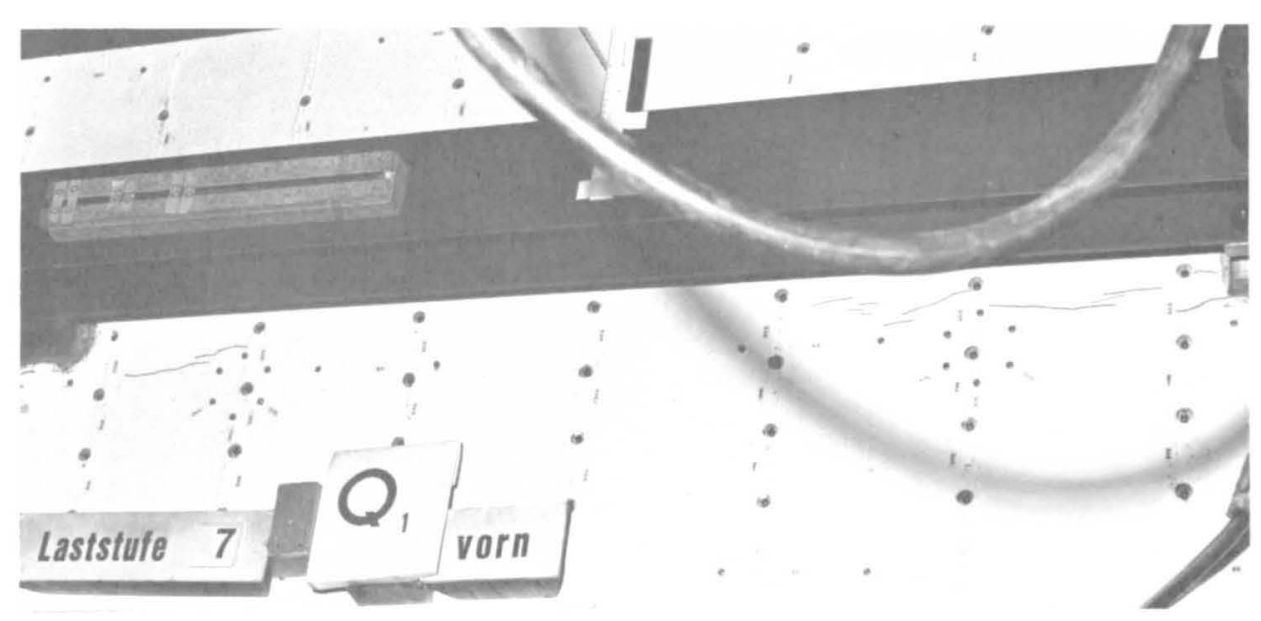

$\mathrm{P}=44 \uparrow \approx \mathrm{P}_{\text {III }}$

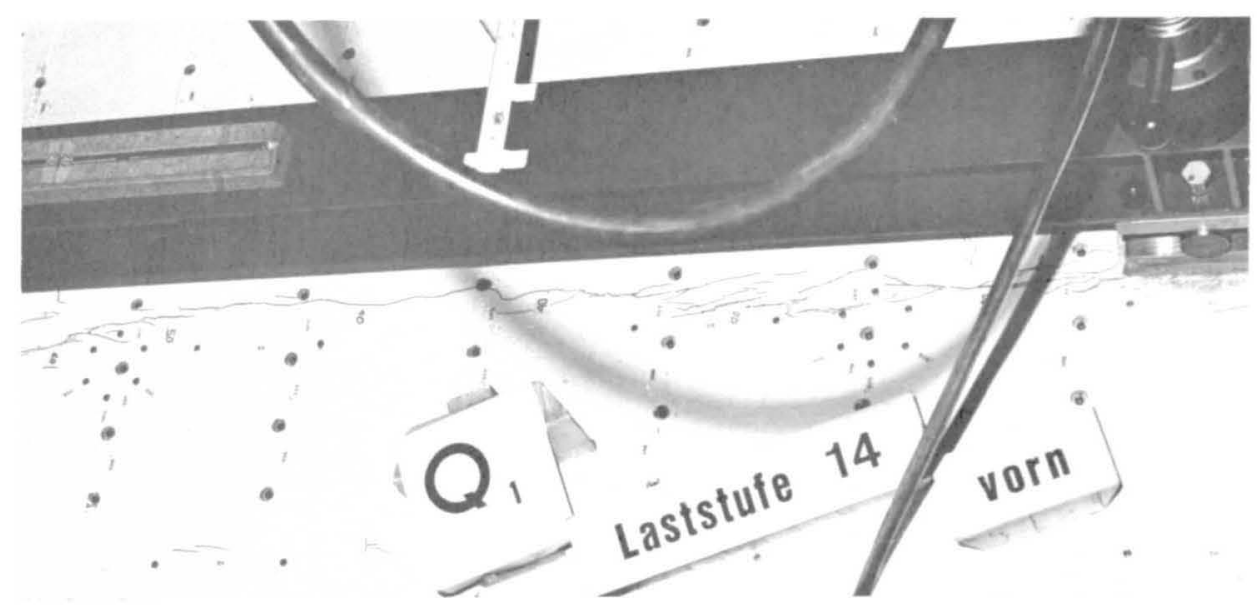

$P_{u}=51.8 t$

Bild 44 : Rissbilder der Platte bei Balken Q1 (Bereich zwischen den zwei Lasten ) 


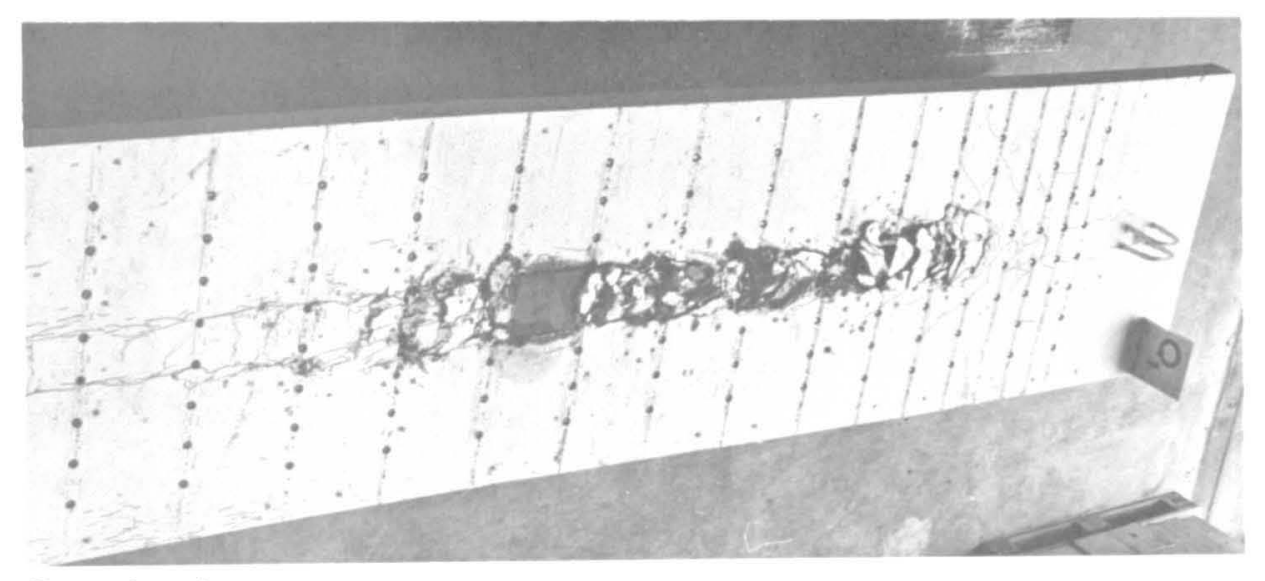

Plattenoberseite

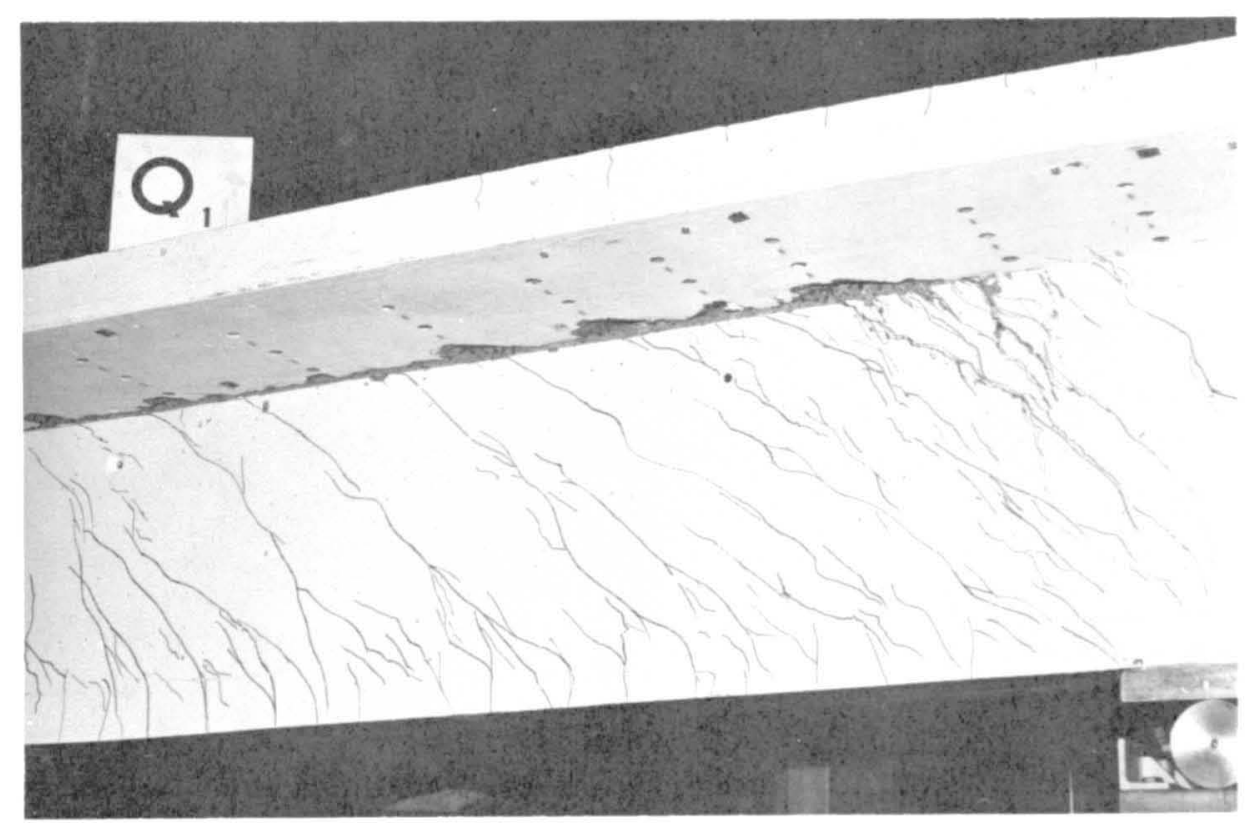

Plattenunterseite

Bild 45: Bruchbilder der Platte bei Balken Q 1 


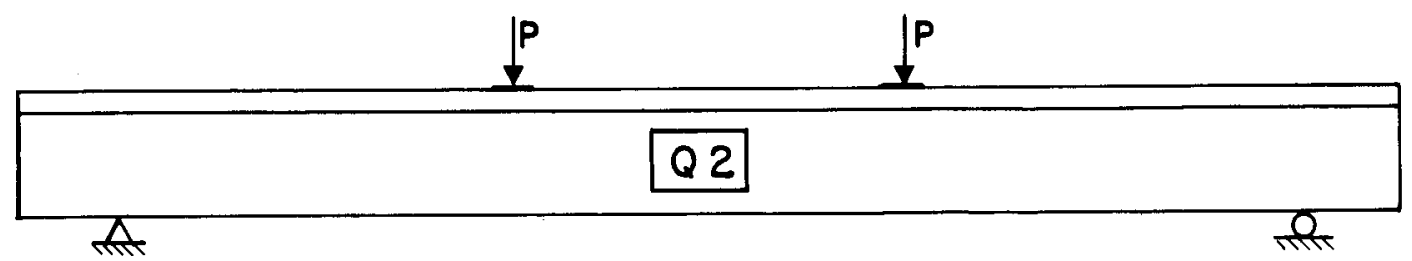

obere $=$ untere Lage der Plattenarmierung

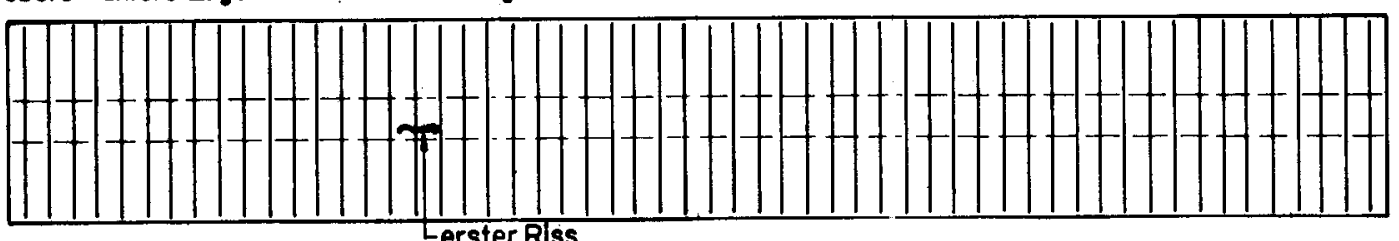

terster Riss

Maximale Rissbreite längs der Platte
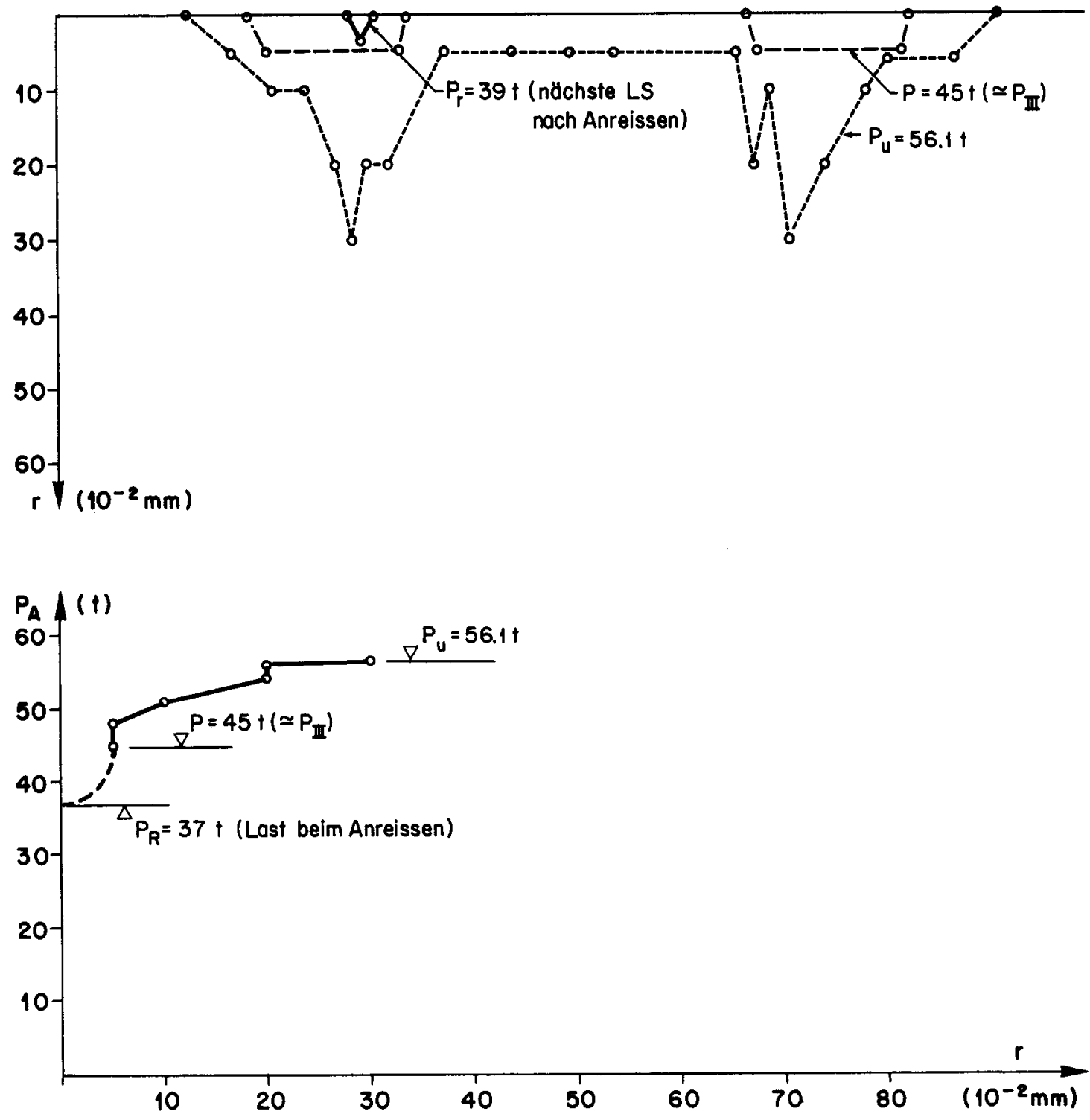

Maximale Rissbreite in Abhängigkeit der Last

Bild 46: Rissbreiten auf der Plattenoberseite bei Balken Q2 


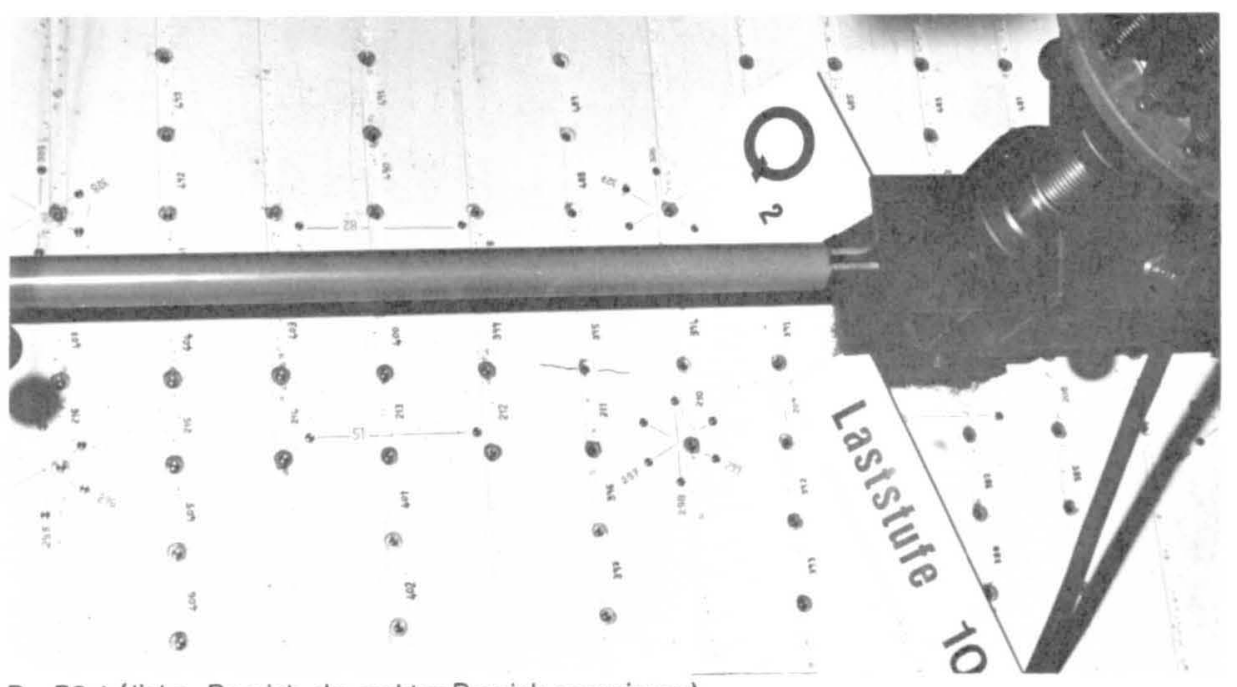

$P_{r}=39 \dagger($ linker Bereich, da rechter Bereich ungerissen)
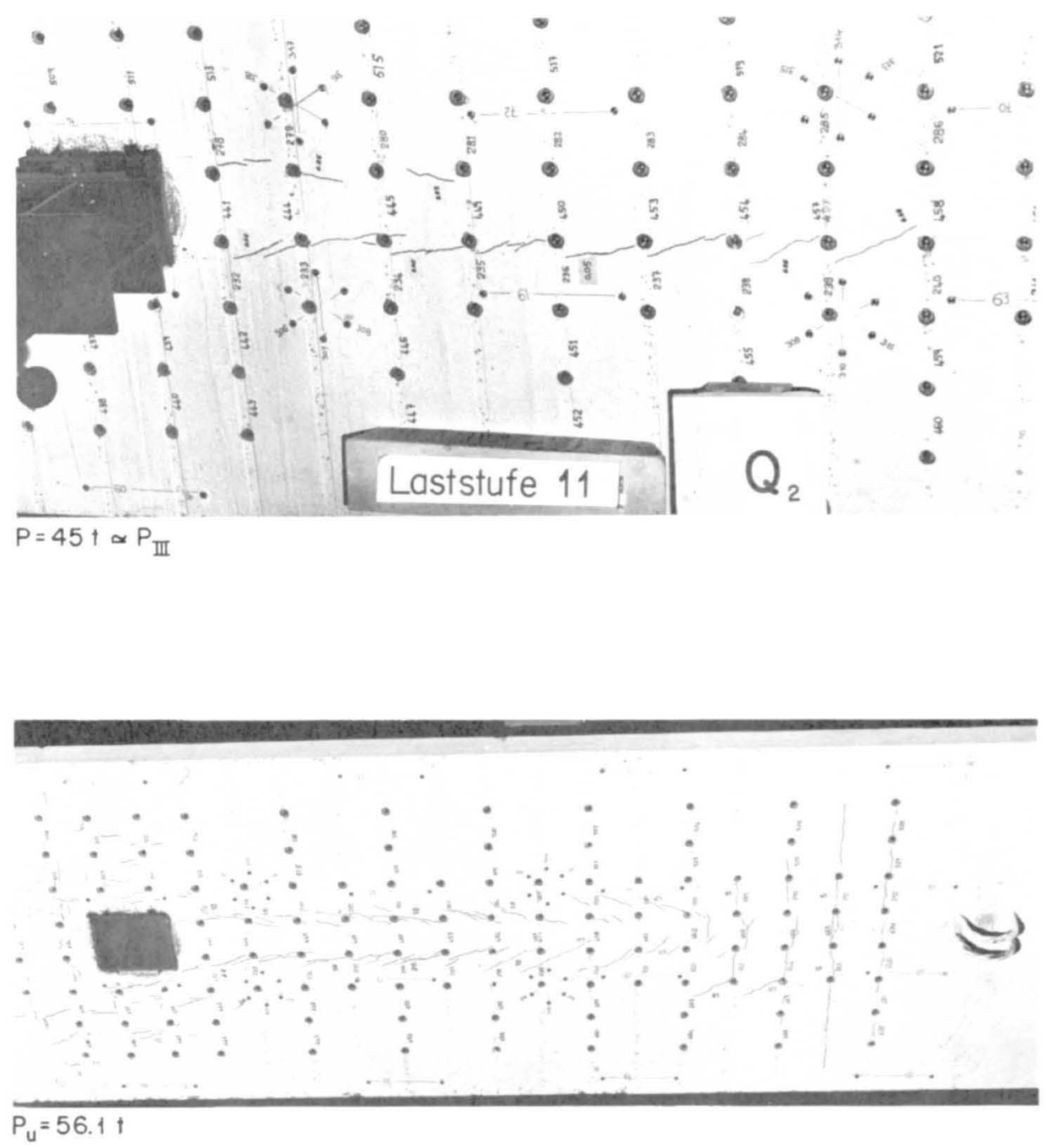

Bild 47 : Rissbilder der Platte bei Balken Q 2 (rechter Bereich). 
$\left.\begin{array}{l}P_{r}=39 t \\ P=45 t\end{array}\right\}$ in diesem Bereich noch keine Risse

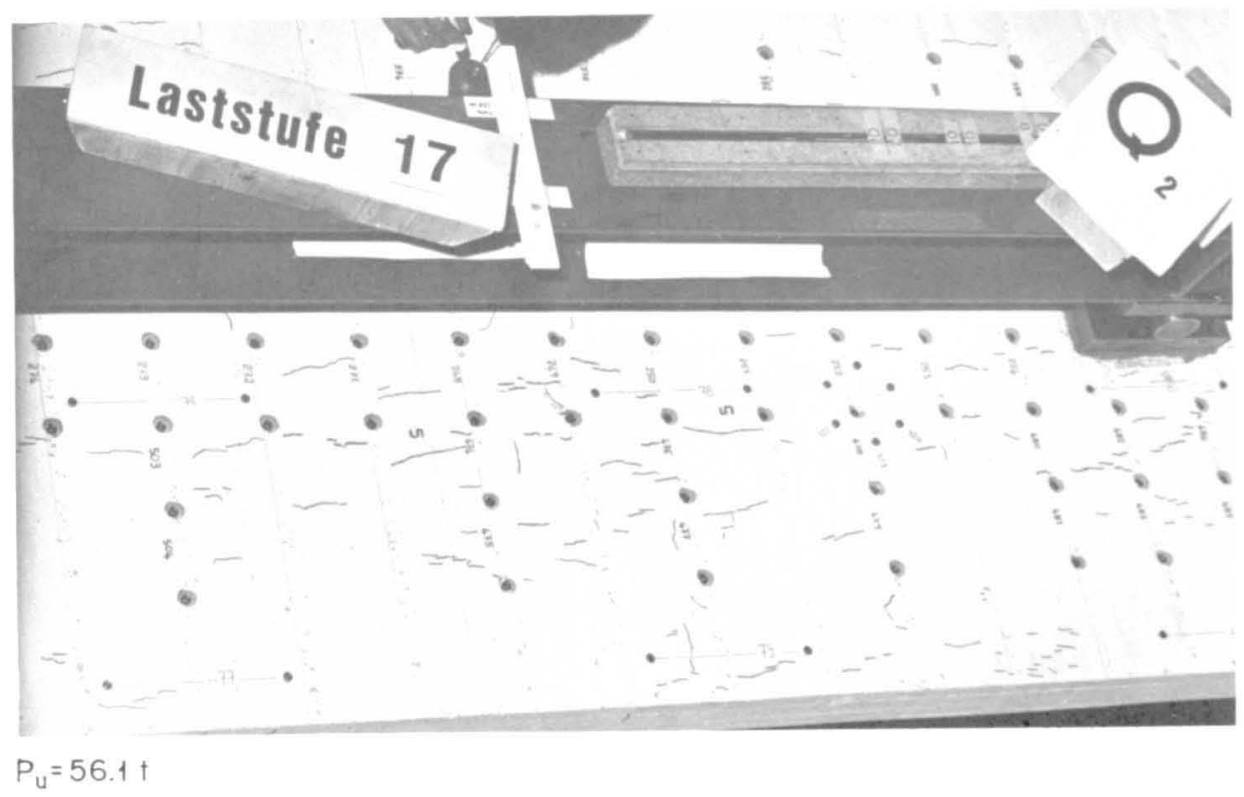

Bild 48 : Rissbild der Platte bei Balken Q2 (Bereich zwischen den zwei Lasten ) 


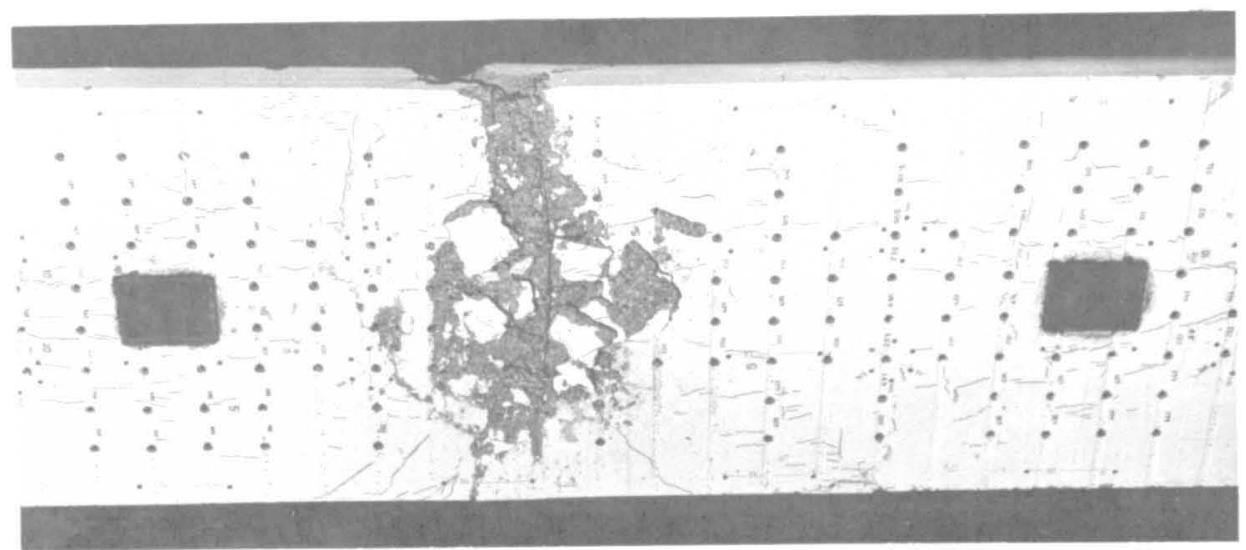

Plattenoberseite

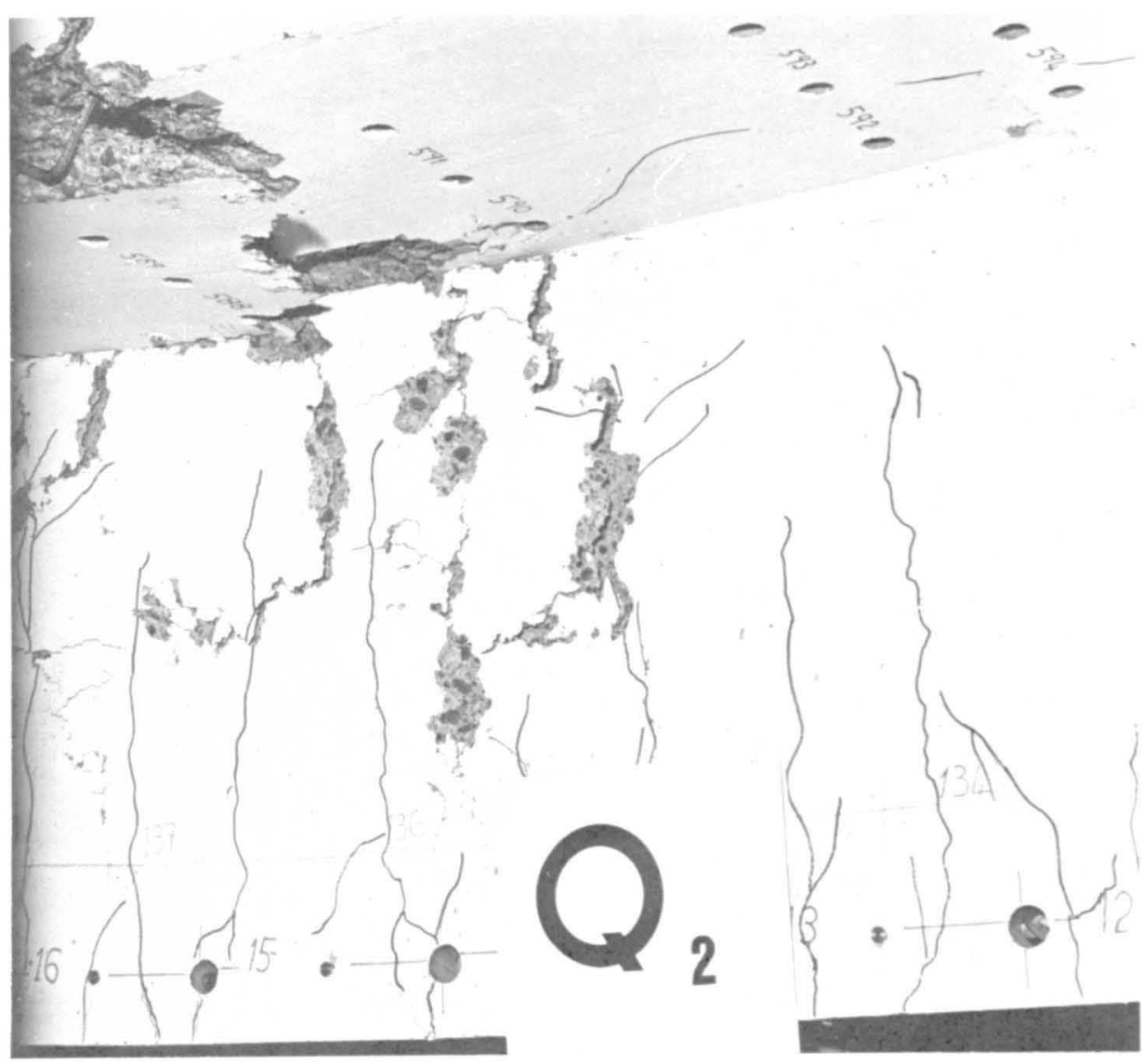

Plattenunterseite

Bild 49: Bruchbilder der Platte bei Balken Q 2 


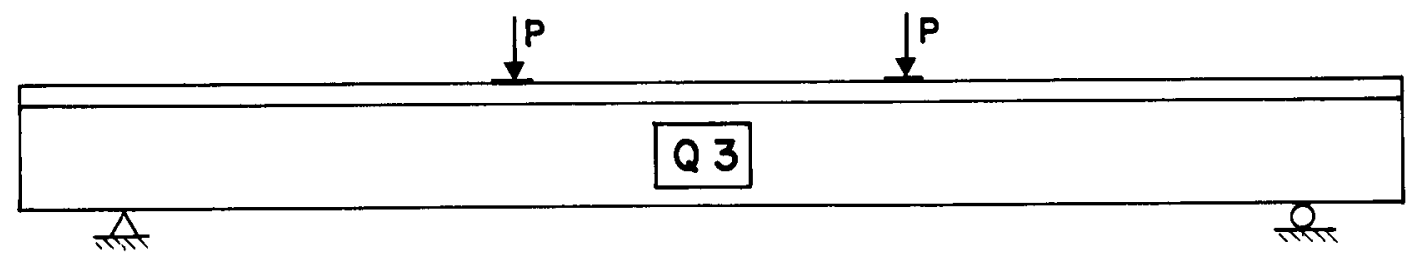

obere Lage der Plattenarmierung

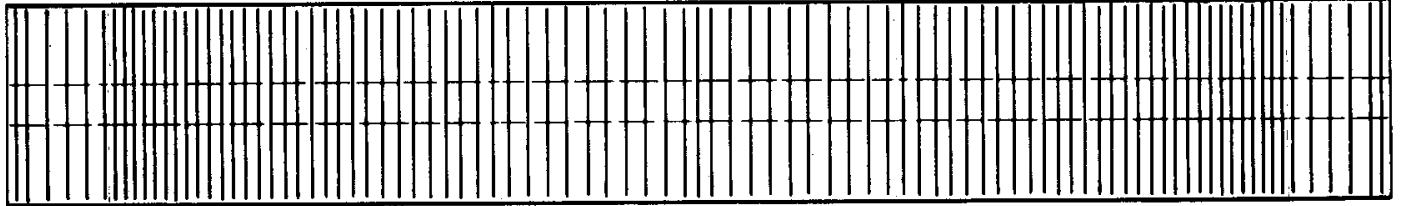

Maximale Rissbreite längs der Platte
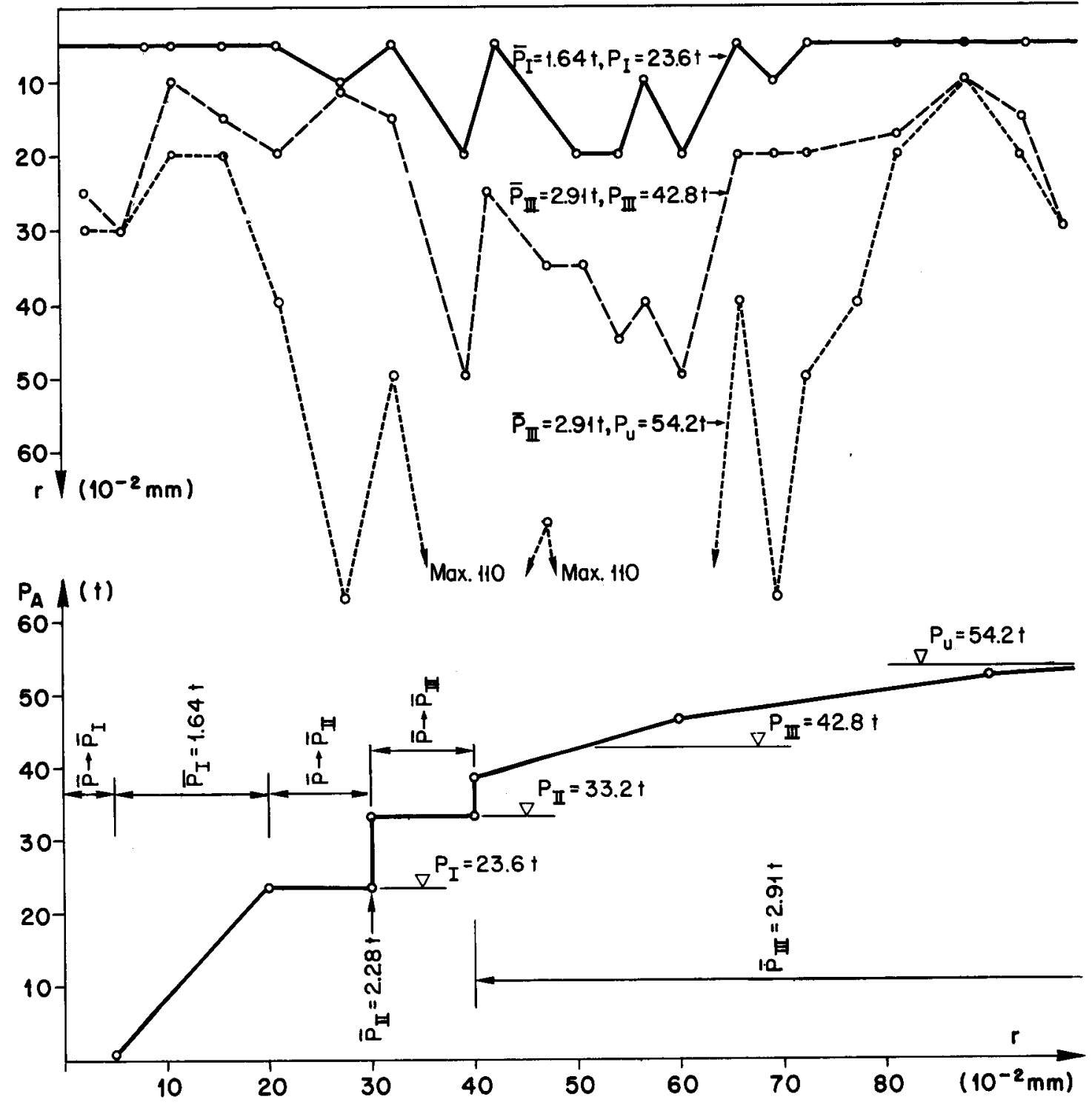

Moximale Rissbreite in Abhängigkeit der Lasł

Bild 50: Rissbreiten auf der Plattenoberseite bei Balken Q 3 


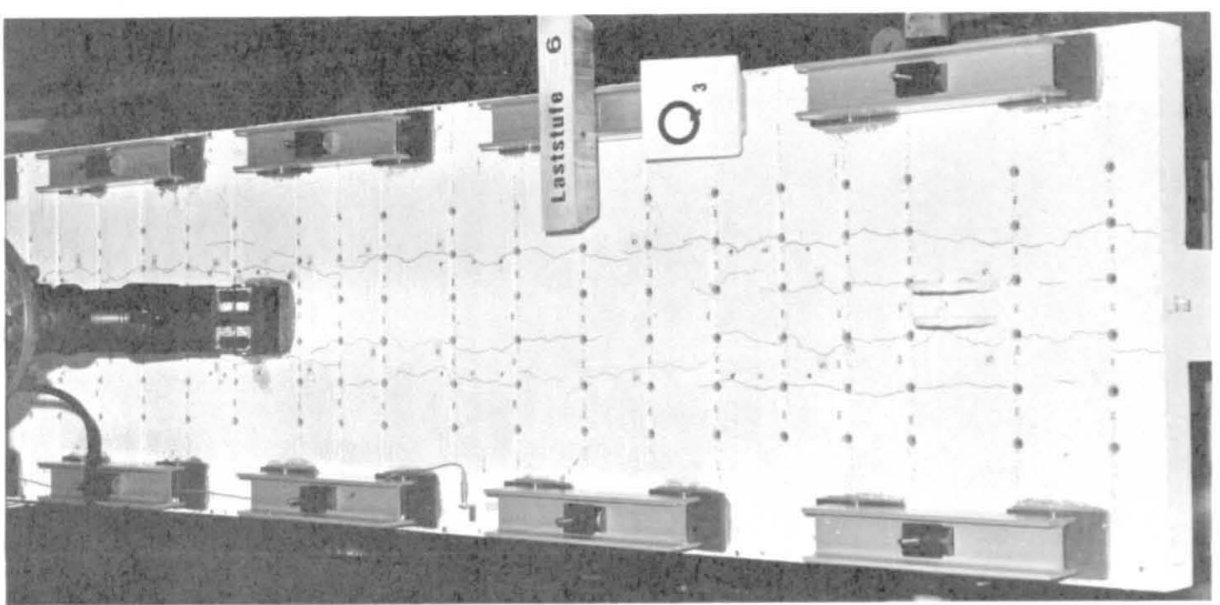

$\bar{P}_{I}=1.64 \dagger, P_{I}=23.6 \dagger$

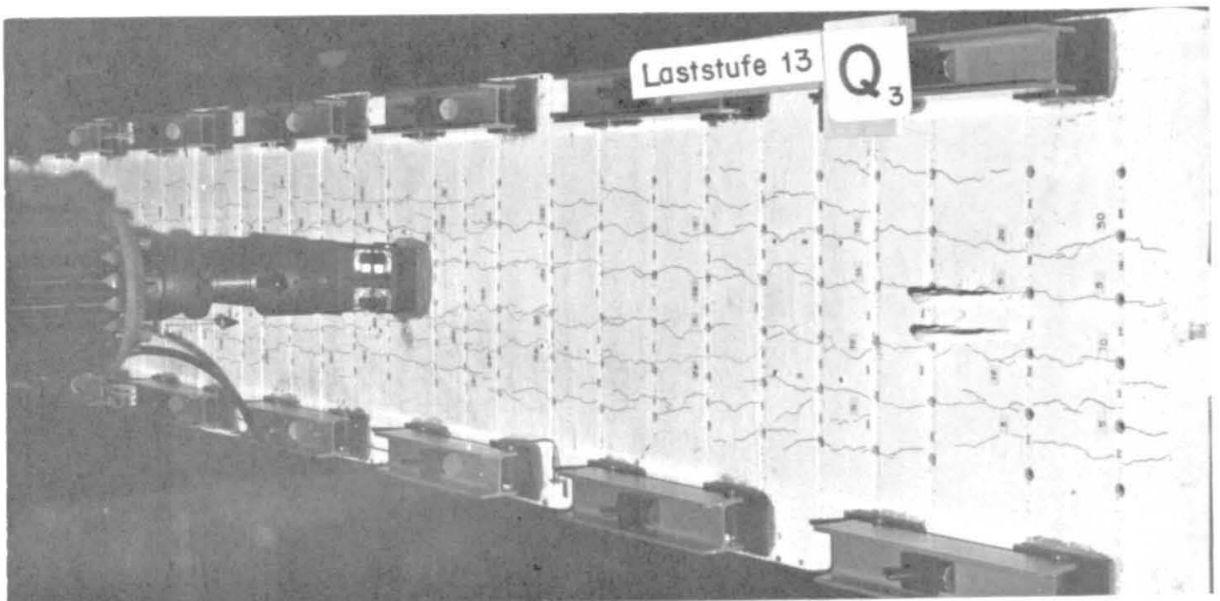

$\bar{P}_{\text {III }}=2.91 \dagger, P=46.8 \dagger$

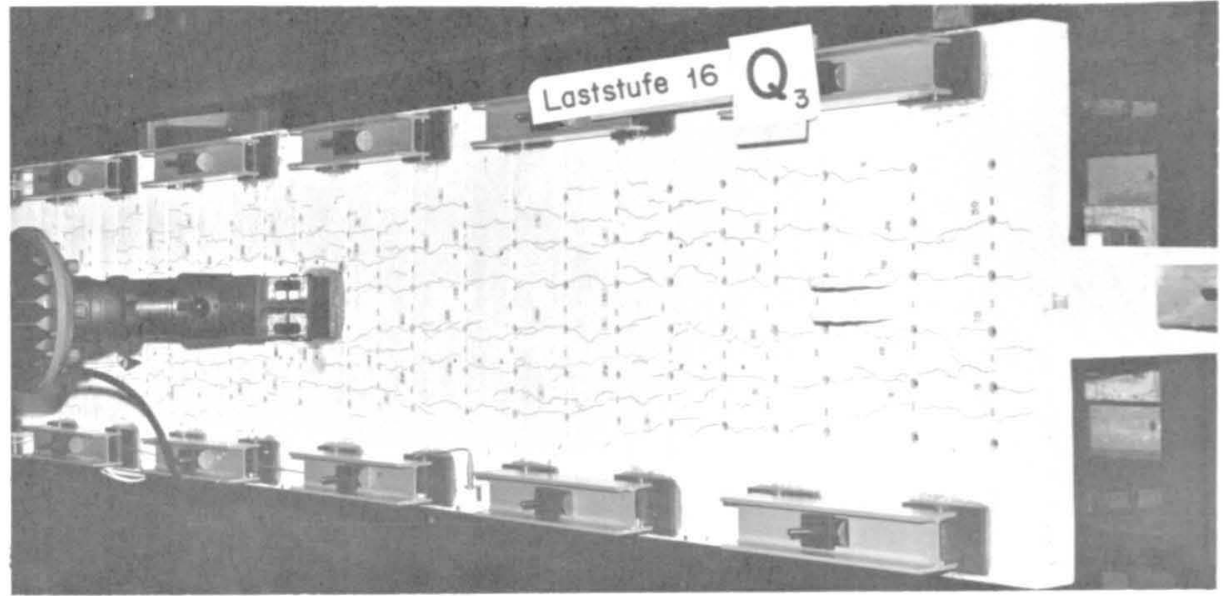

$\bar{P}_{\text {III }}=2.91 \mathrm{t}, P_{\mathrm{u}}=54.2$

Bild 51 : Rissbilder der Platte bei Balken Q 3 (rechter Bereich) 

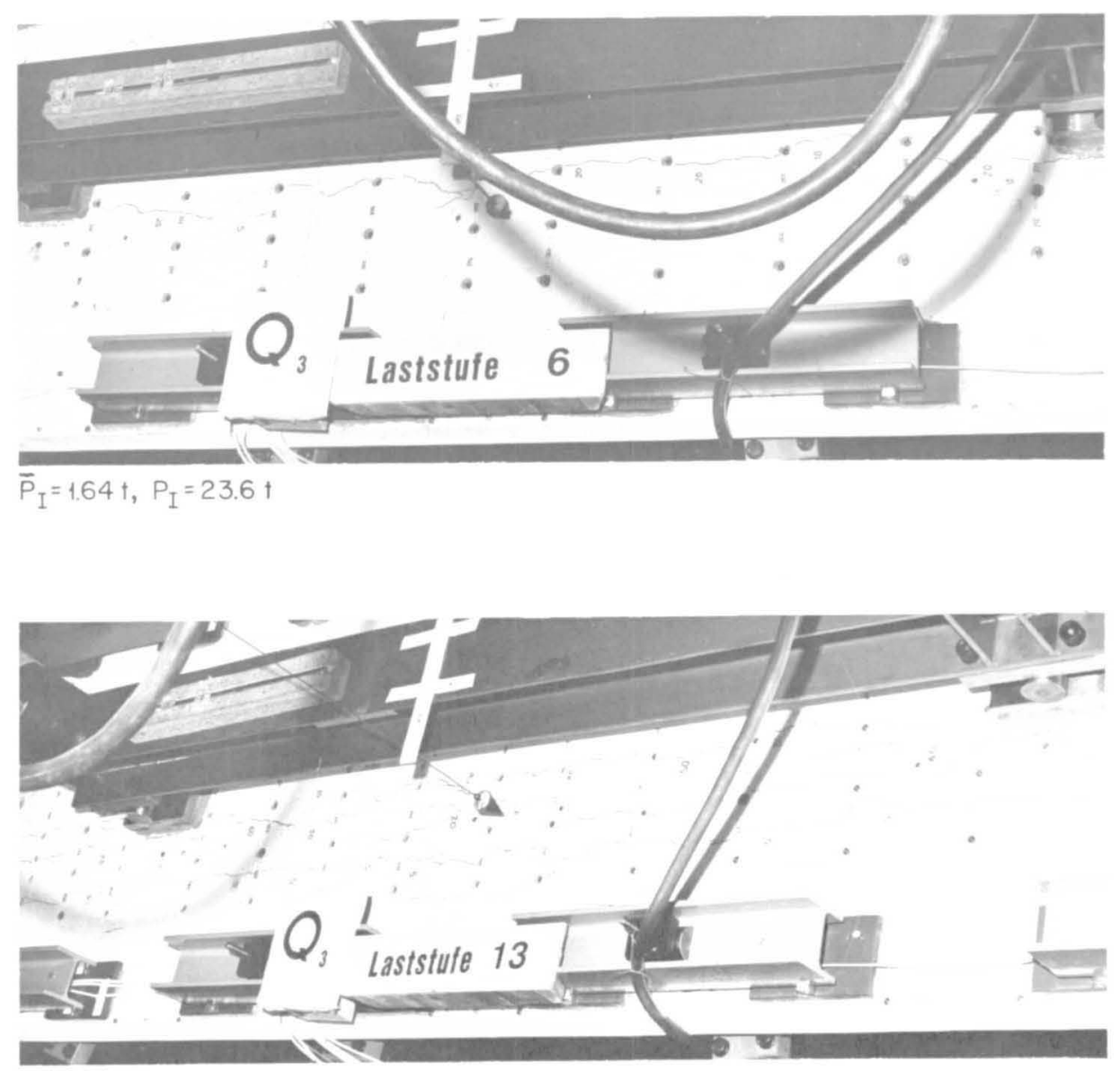

$\overline{\mathrm{P}}_{\text {III }}=2.91 \dagger, \mathrm{P}=46.8 \dagger$

Bild 52 : Rissbilder der Platte bei Balken Q3 (Bereich zwischen den zwei Lasten)

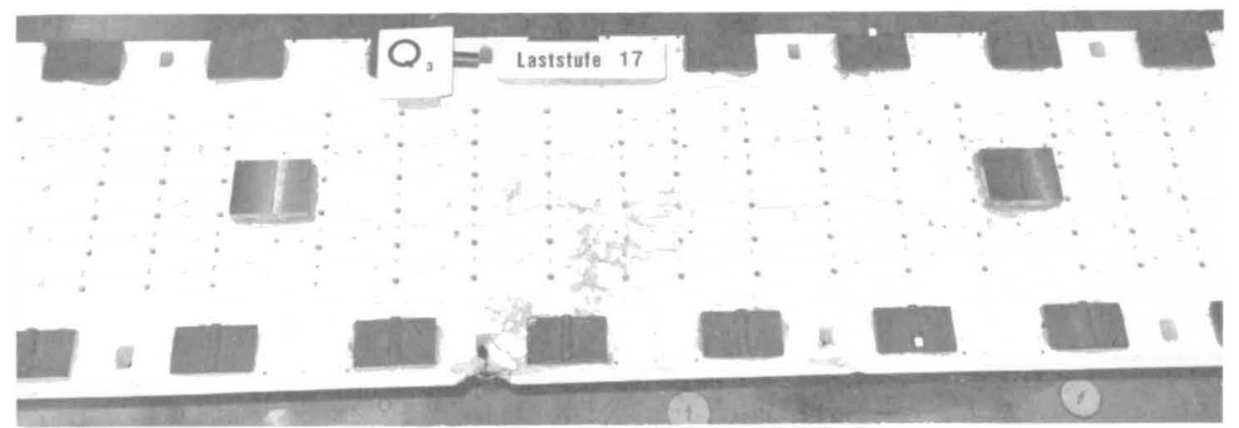

Bild 53: Bruchbild der Platte bei Balken Q 3 


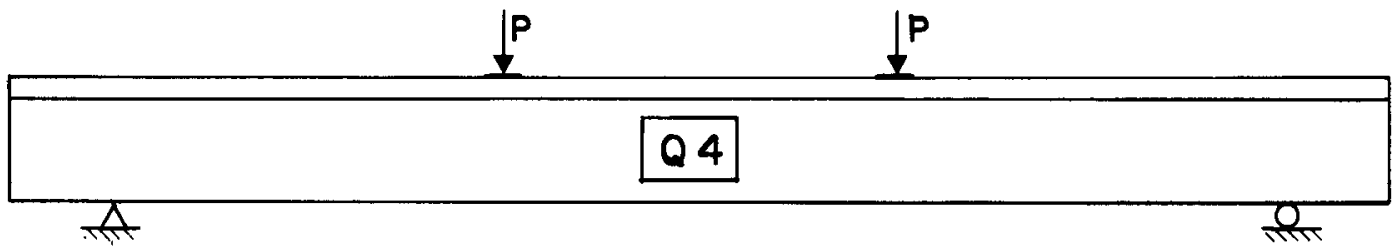

obere Loge der Plattenarmierung

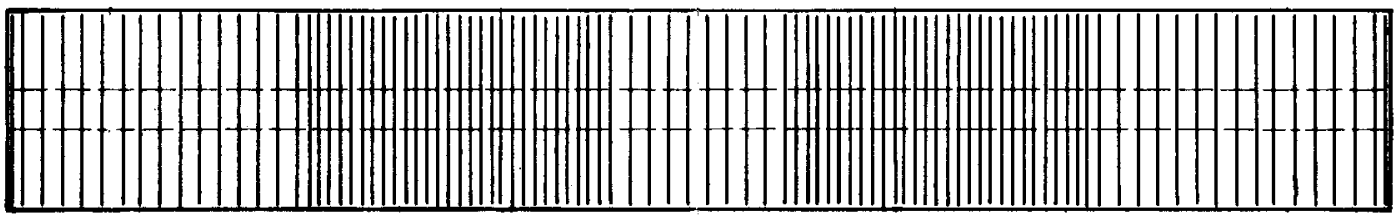

Maximale Rissbreite löngs der Platte
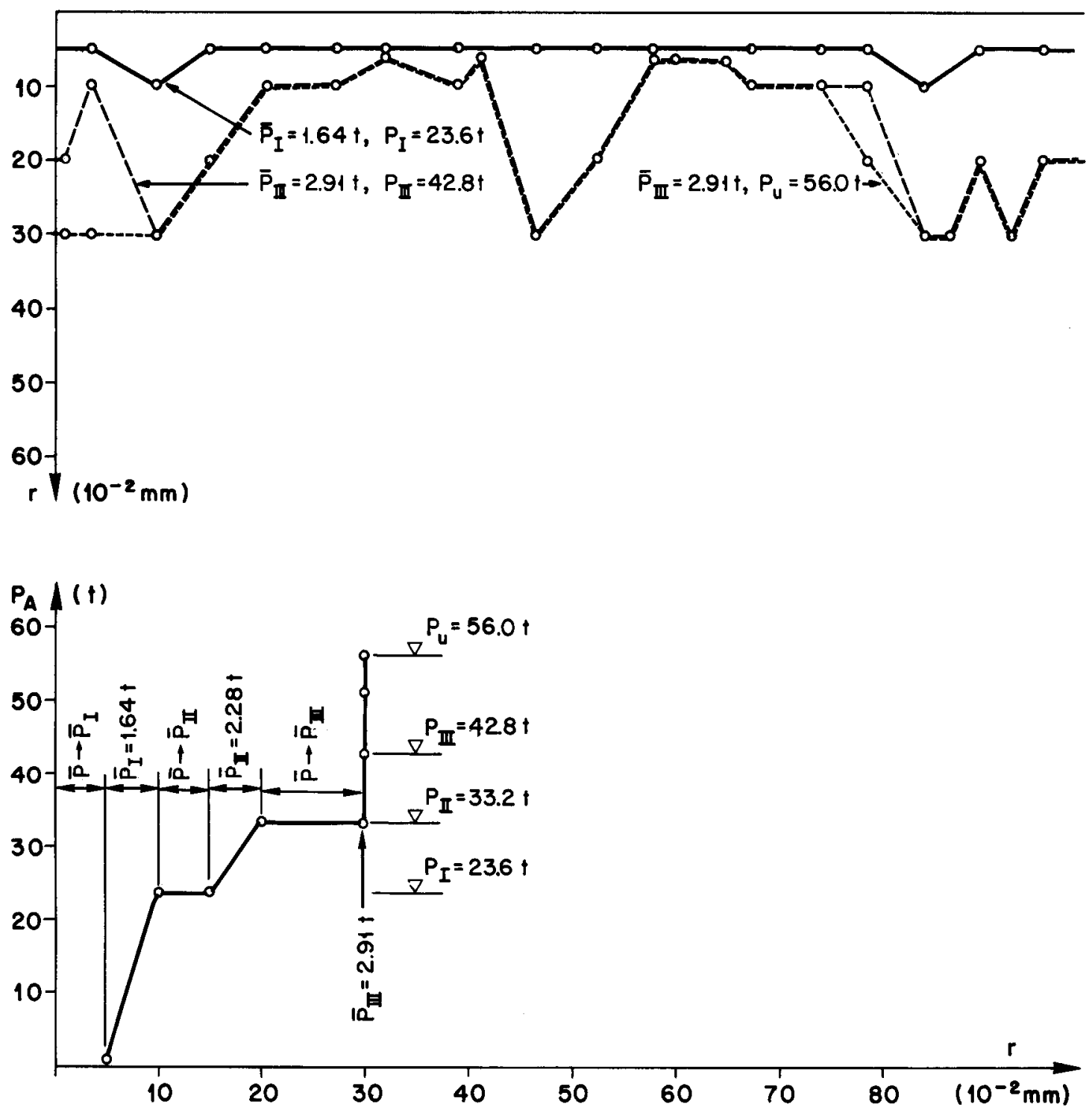

Maximale Rissbreite in Abhängigkeit der Lasł

Bild 54: Rissbreiten auf der Plattenoberseite bei Balken Q 4 


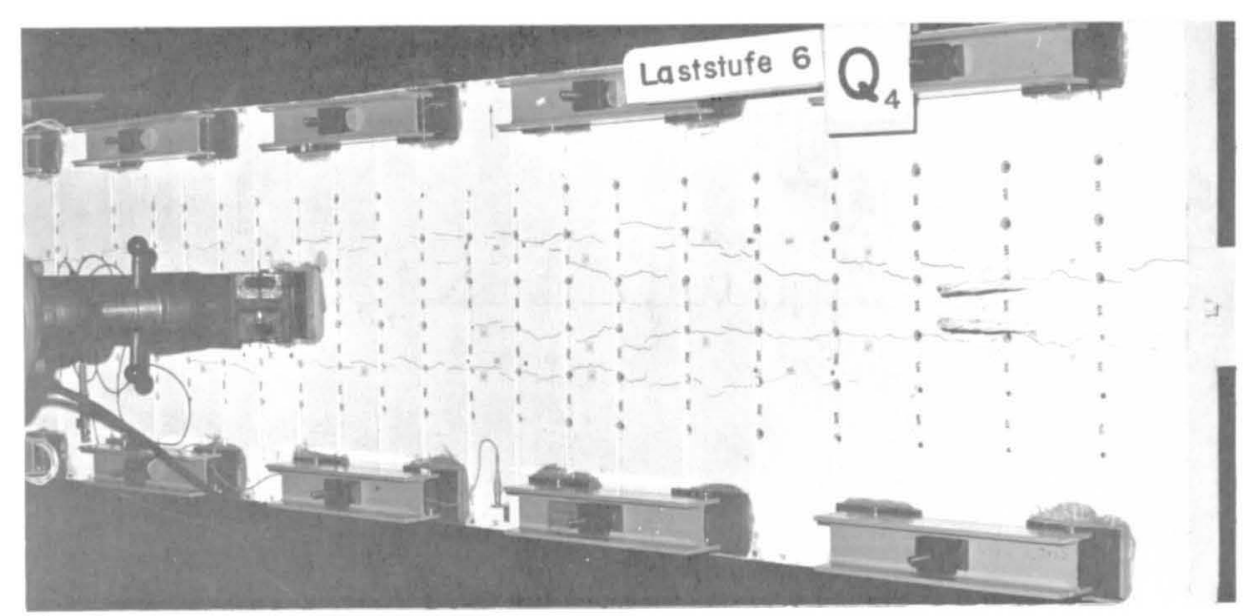

$\bar{P}_{I}=1.64 t, P_{I}=23.6 t$

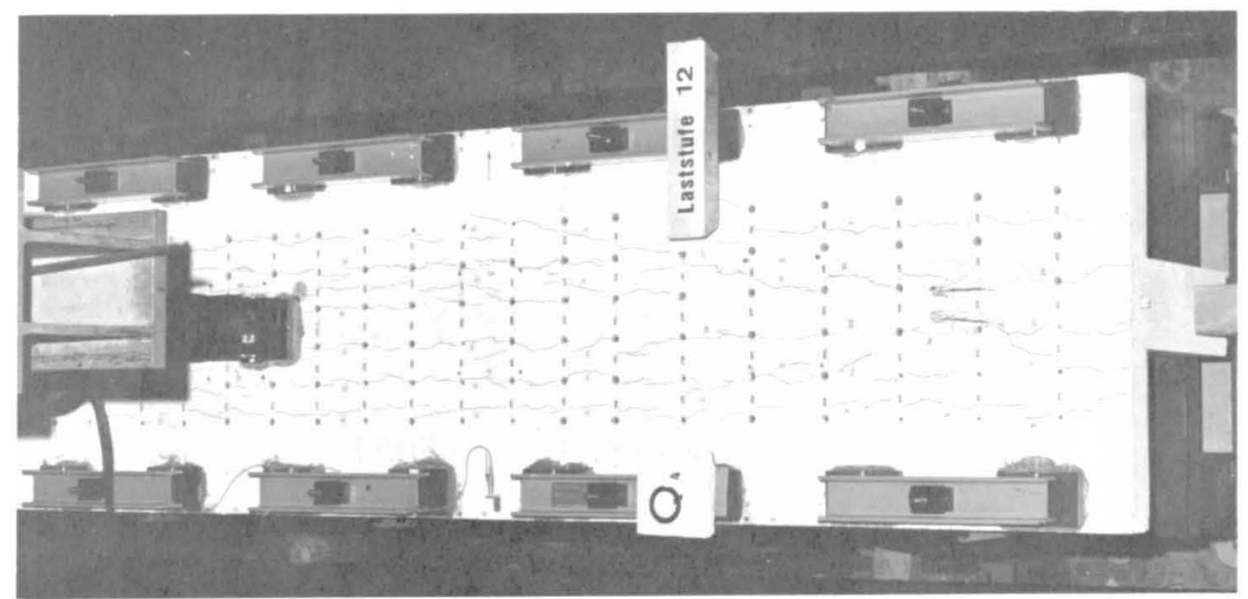

$\bar{P}_{\text {III }}=2.91 \uparrow, P_{\text {II }}=42.8 t$

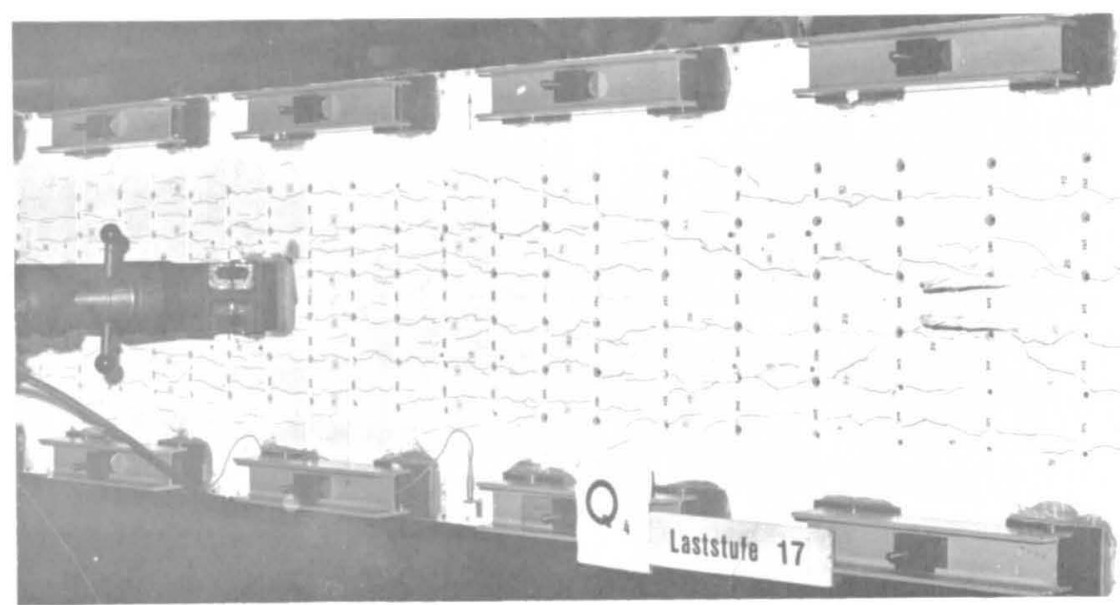

$\bar{P}_{\text {III }}=2.91 \dagger, P_{u}=56 \dagger$

Bild 55: Rissbilder der Platte bei Balken Q 4 (rechter Bereich) 


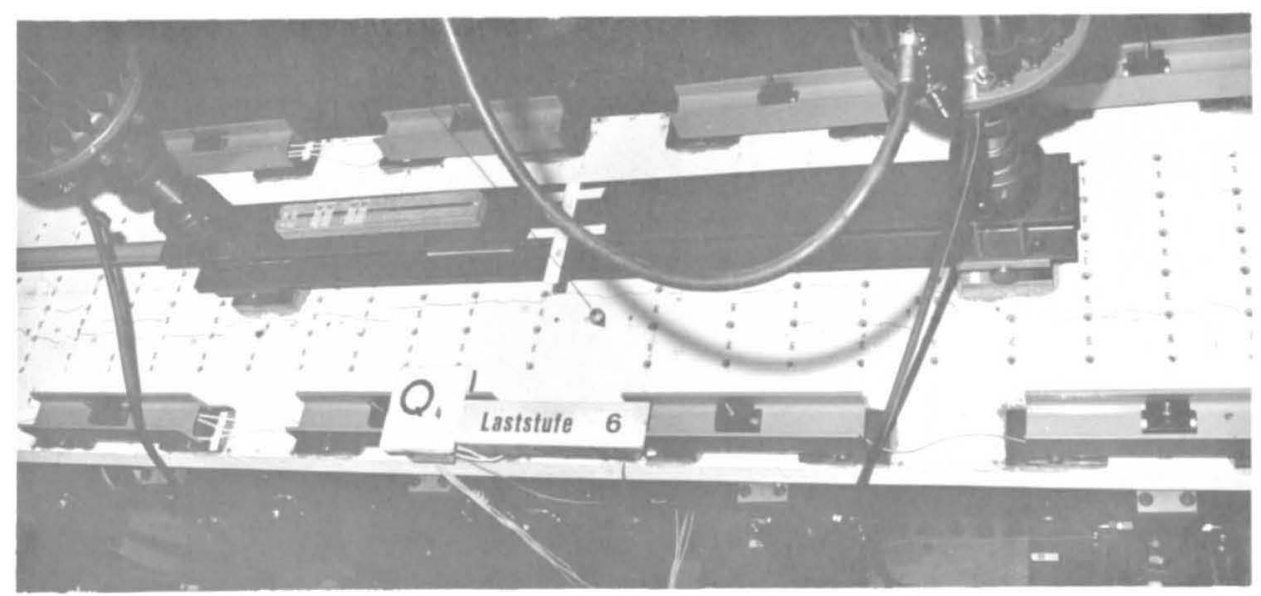

$\bar{P}_{I}=1.64 t, P_{I}=23.6 t$

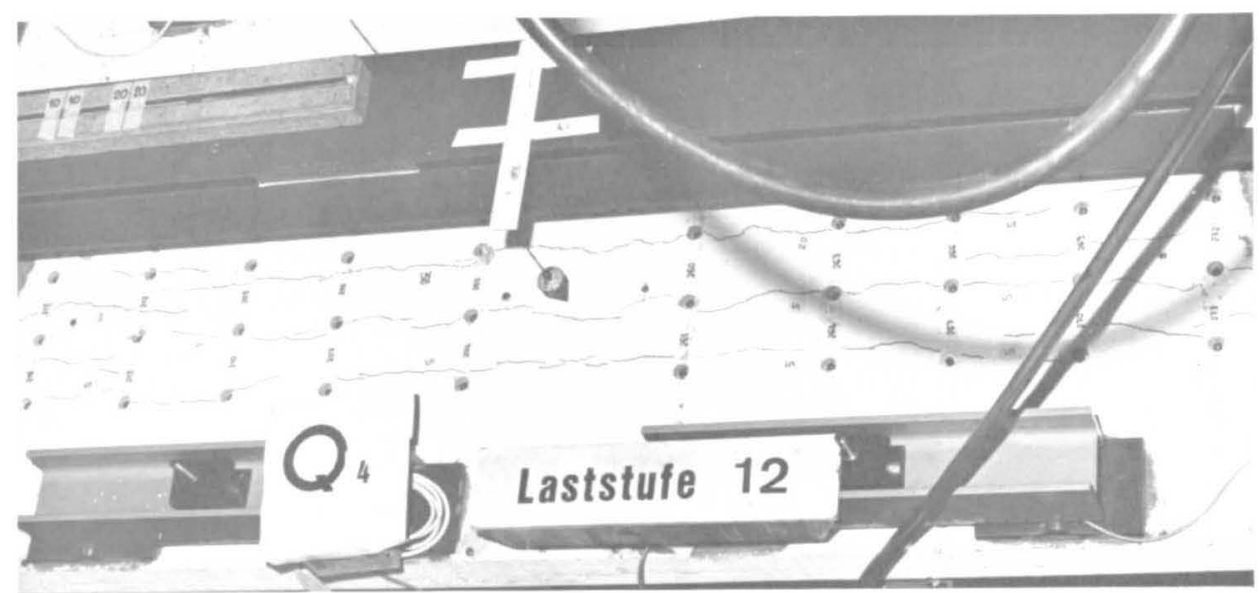

$\overline{\mathrm{P}}_{\text {III }}=2.91 \dagger, \mathrm{P}_{\text {III }}=42.8 \dagger$

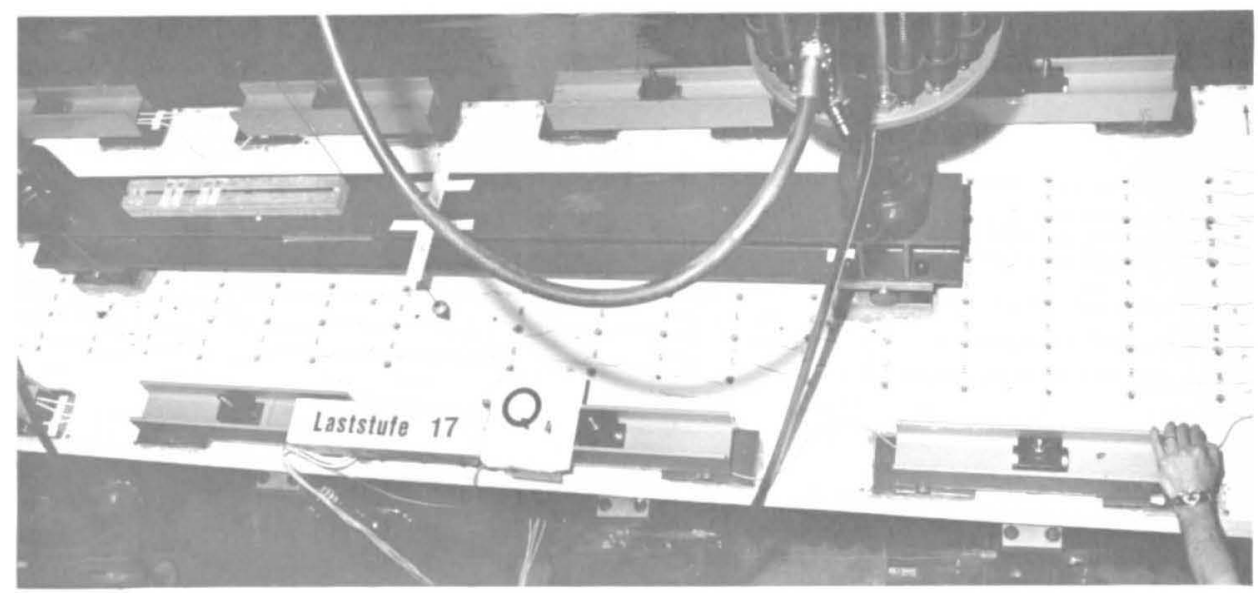

$\bar{P}_{\text {III }}=2.91 \dagger, P_{u}=56 \dagger$

Bild 56: Rissbilder der Platte bei Balken Q 4 (Bereich zwischen den zwei Lasten ) 


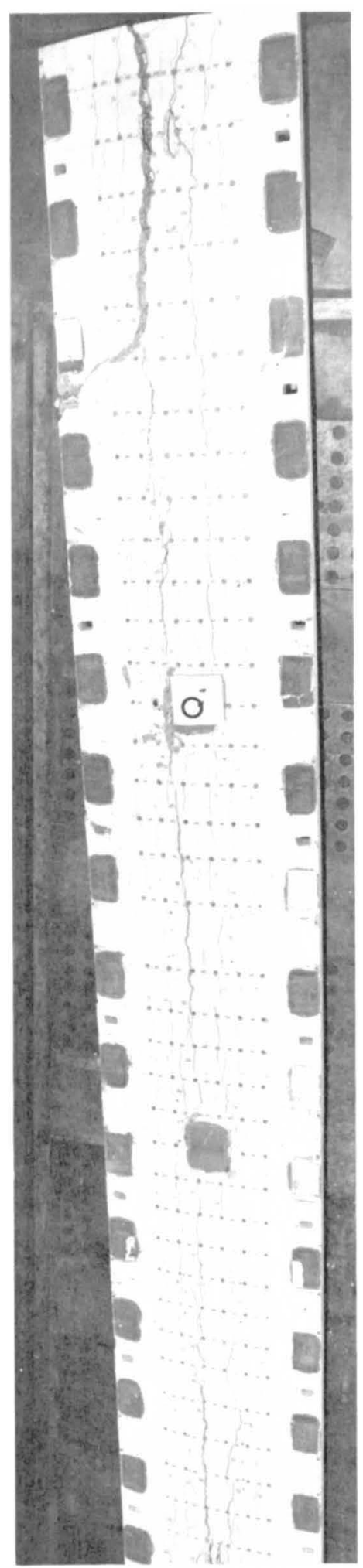

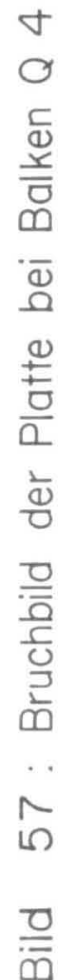




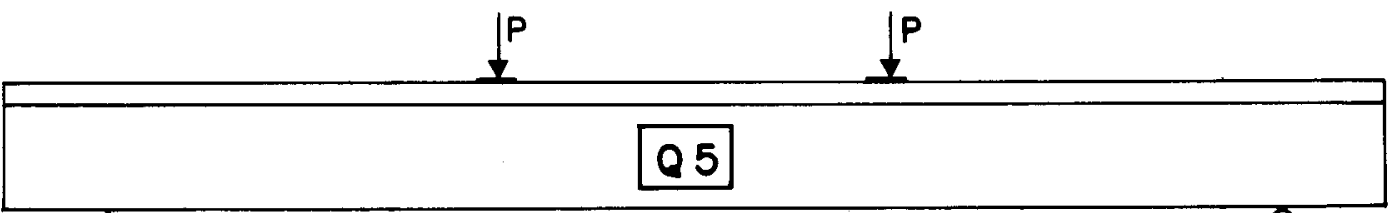

An

obere Lge der Plattenarmierung

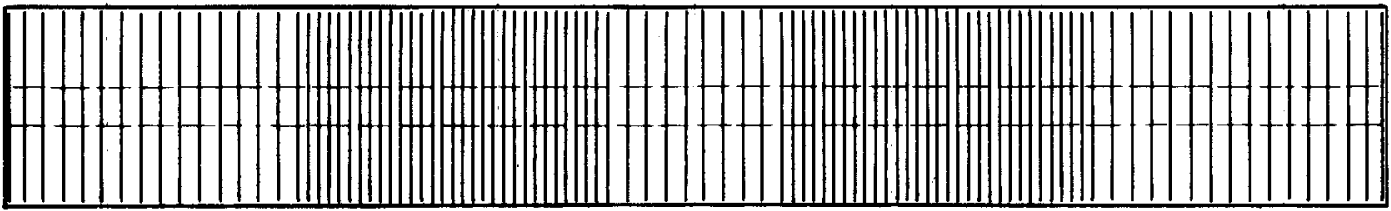

Maximale Rissbreite längs der Platte
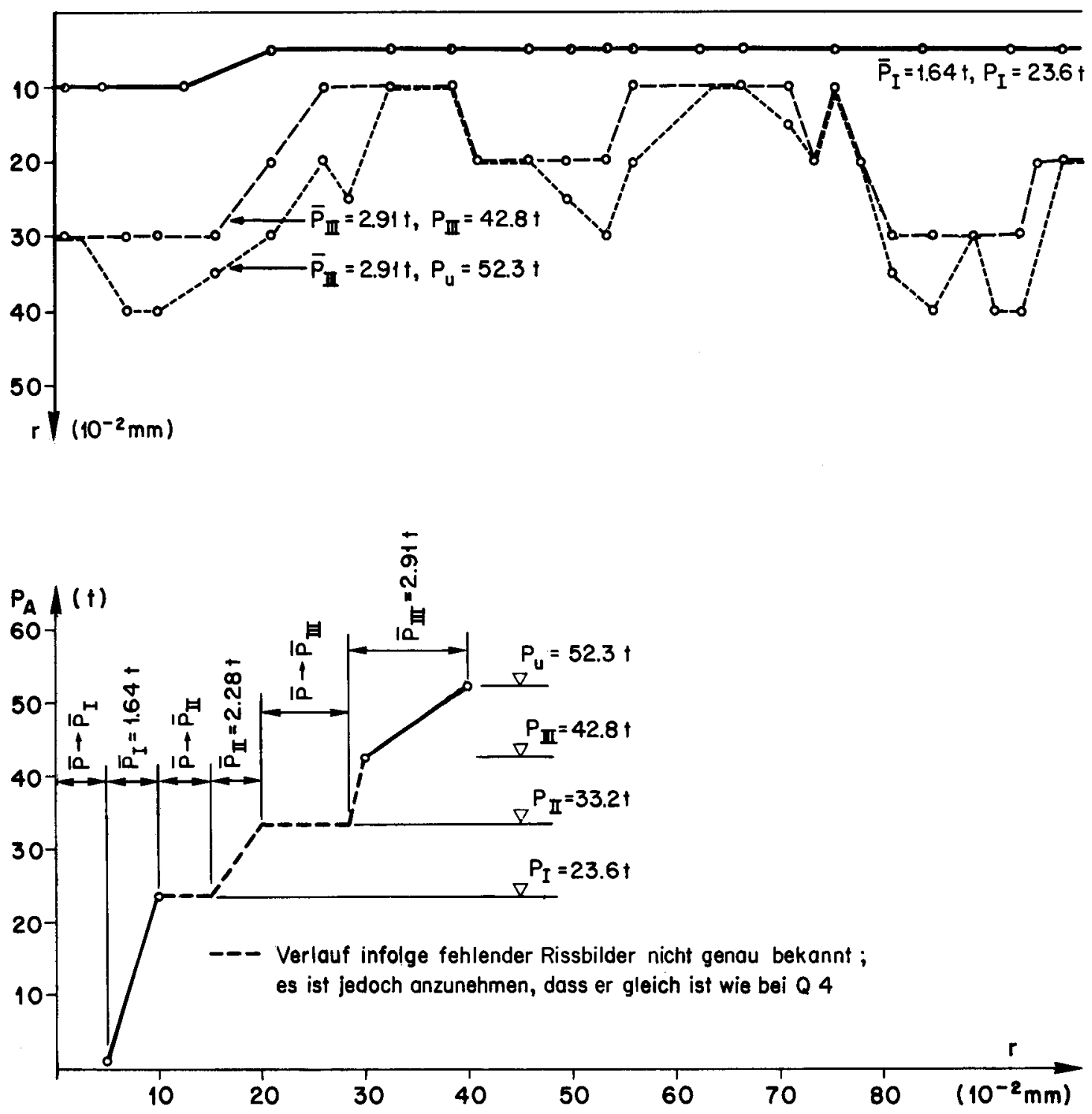

Maximale Rissbreite in Abhöngigkeit der Lasł

Bild 58 : Rissbreiten auf der Plattenoberseite bei Balken Q 5 


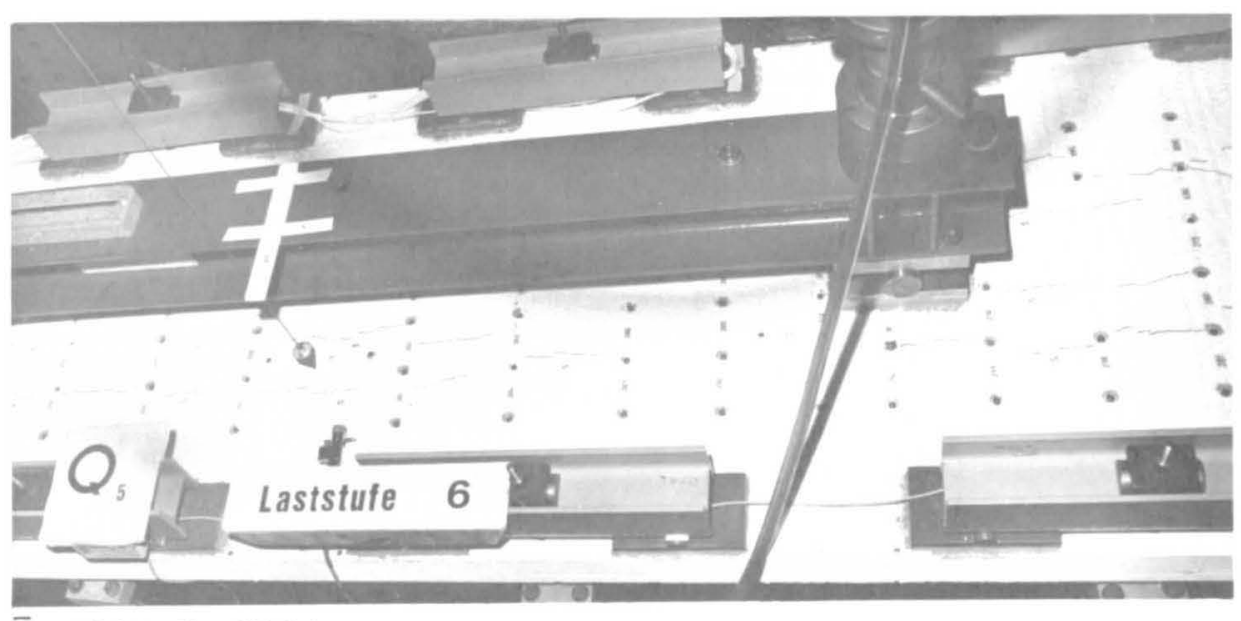
$\bar{P}_{I}=1.64 t, P_{I}=23.6 t$

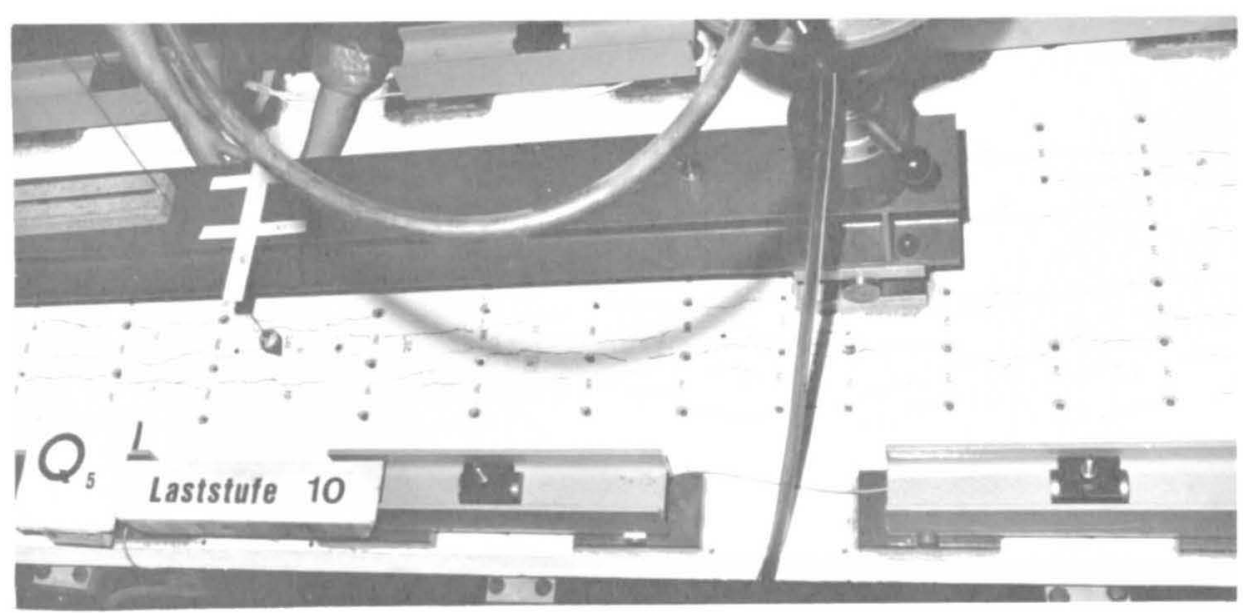

$\bar{P}_{\text {III }}=2.91 \dagger, \quad P_{\text {III }}=42.8 \dagger$

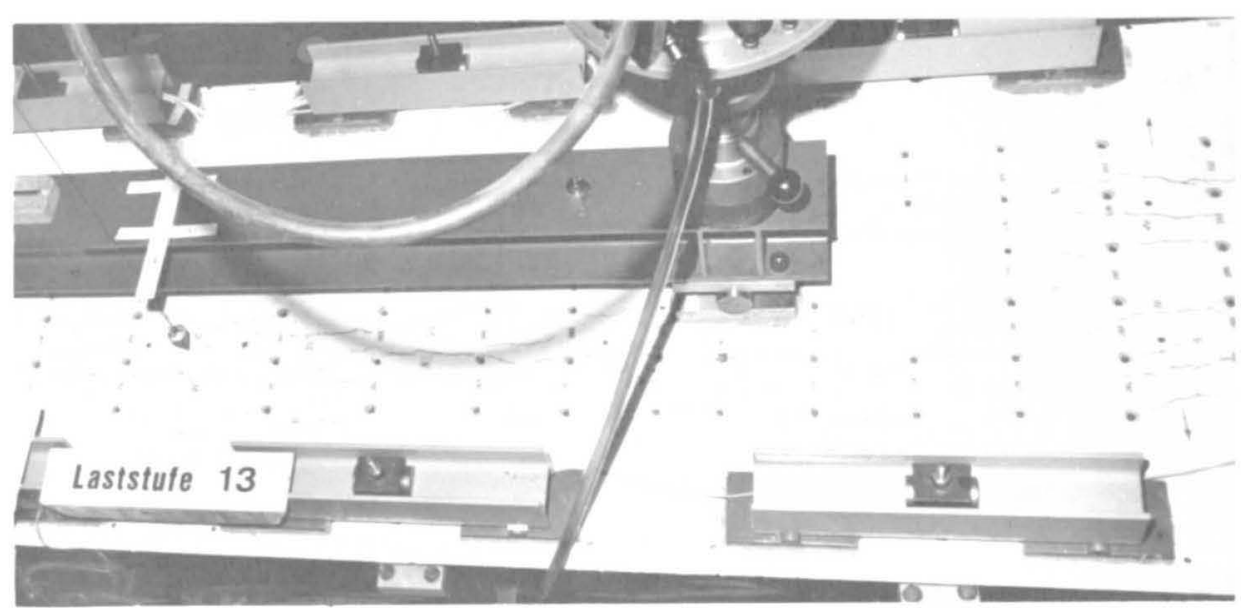

$\bar{P}_{\text {III }}=2.911, \quad P_{u}=52.3 \dagger$

Bild 60: Rissbilder der Platte bei Balken Q 5 (Bereich zwischen den zwei Lasten ) 

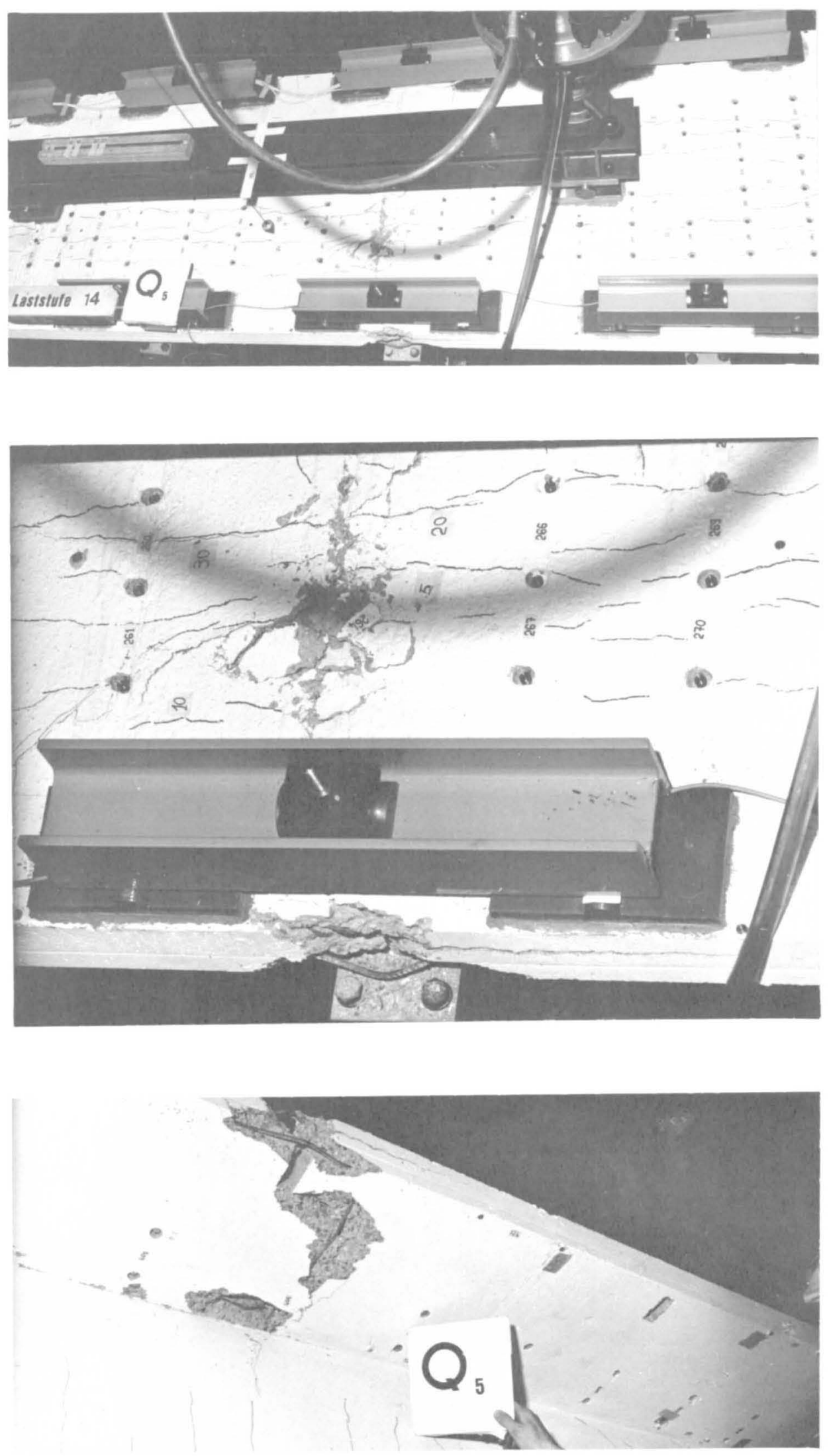

Bild 61: Bruchbilder der Platte bei Balken Q 5 

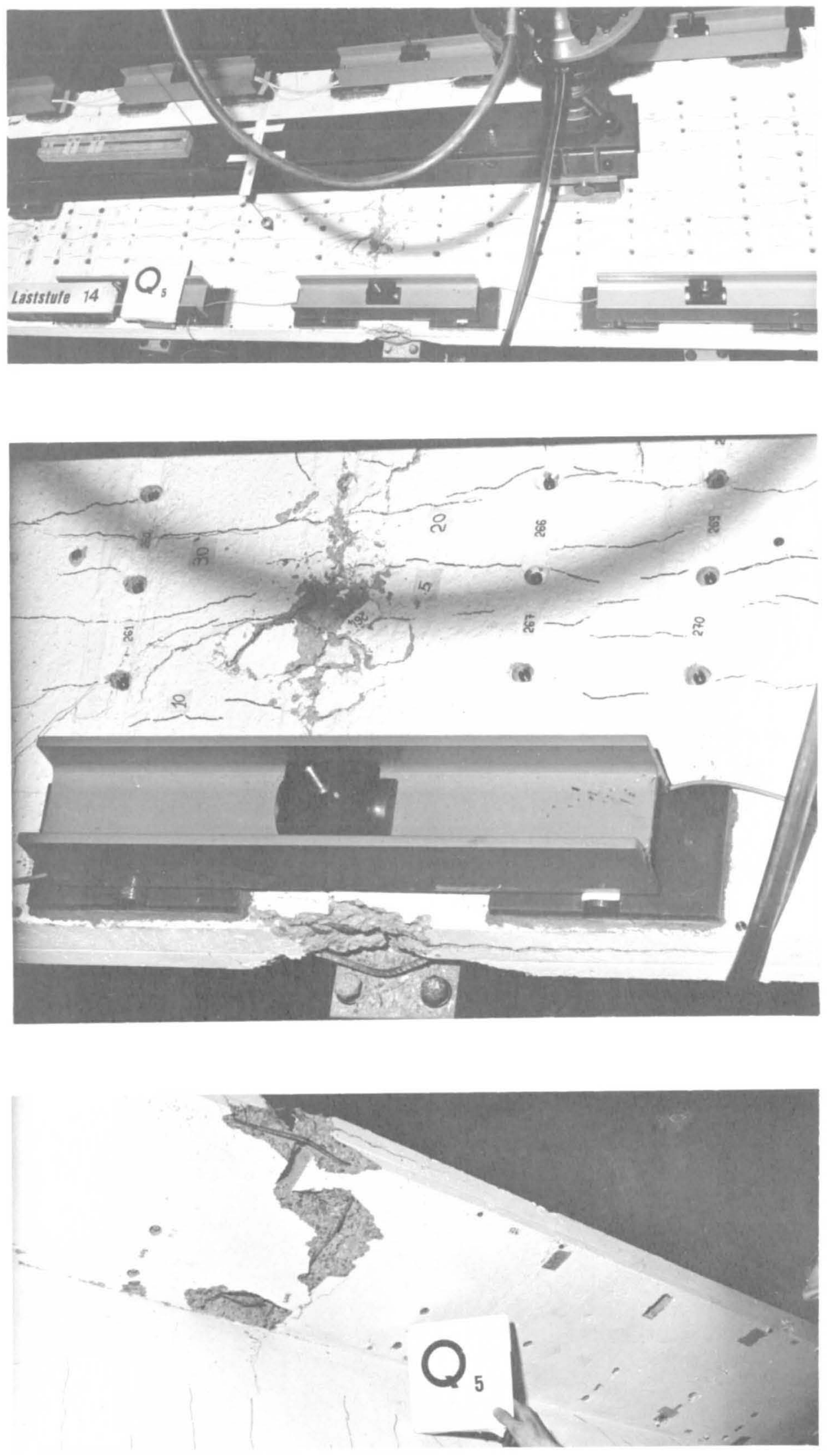

Bild 61: Bruchbilder der Platte bei Balken Q 5 


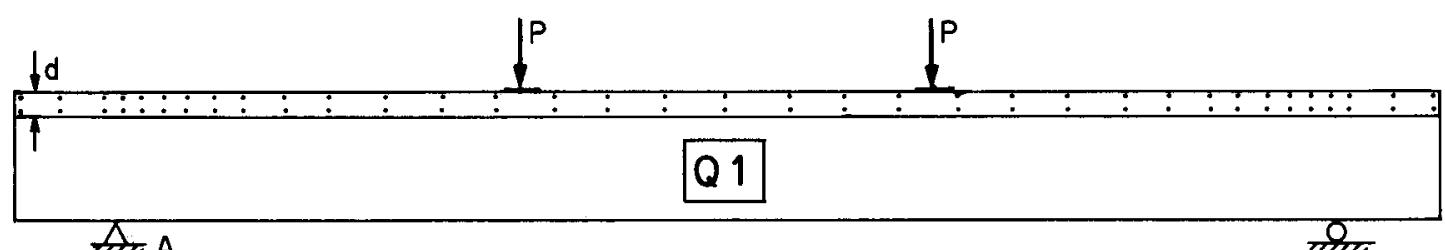

मीलि A

min?
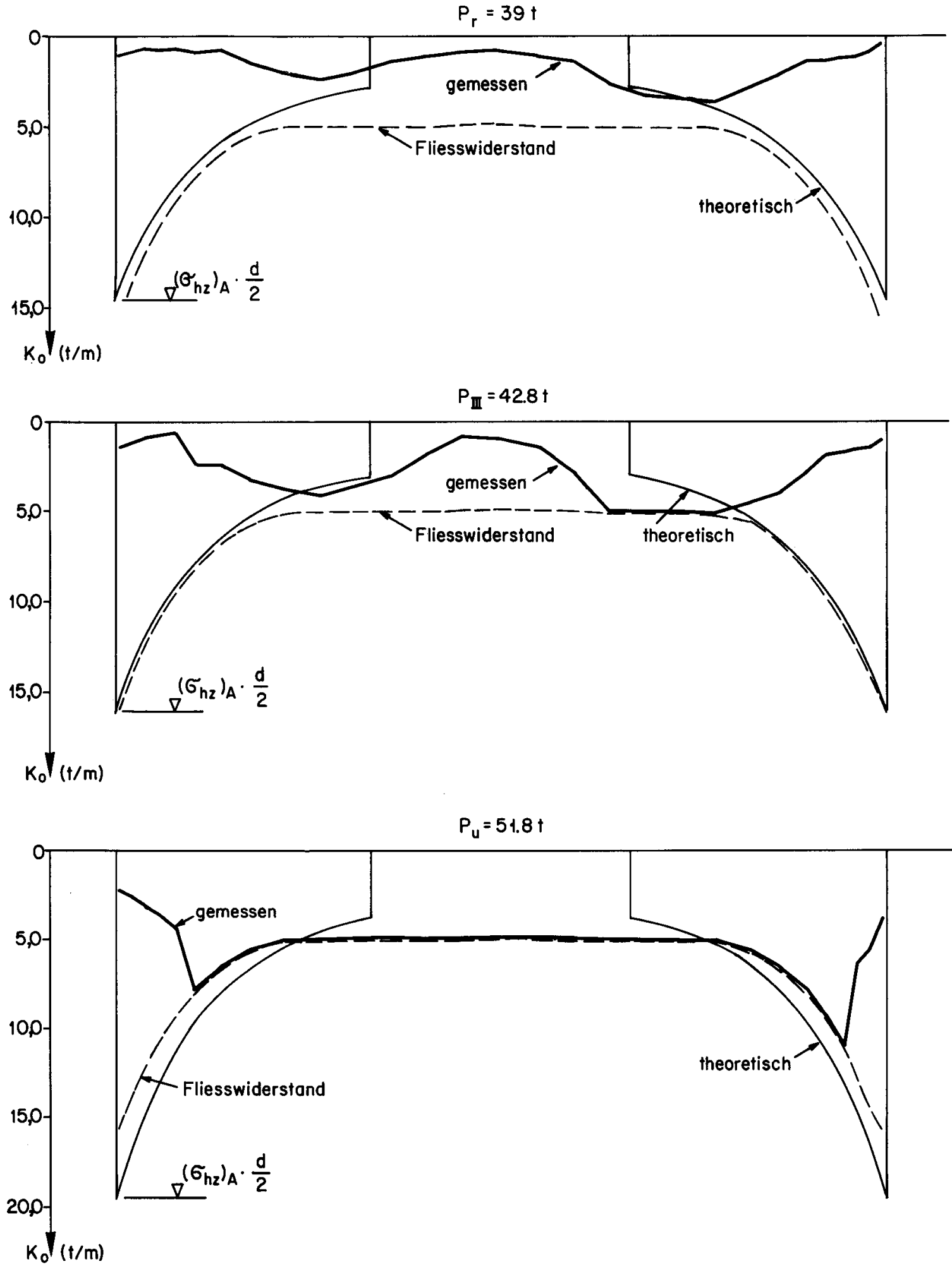

Bild 62: Theoretische und gemessene Zugkraft pro Längeneinheit in der oberen Lage der Plattenarmierung bei Balken Q 1 


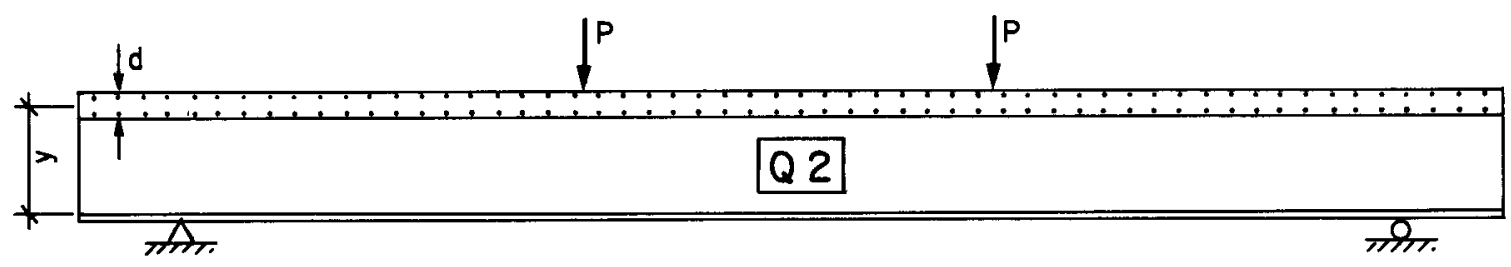

$P_{r}=39 t$
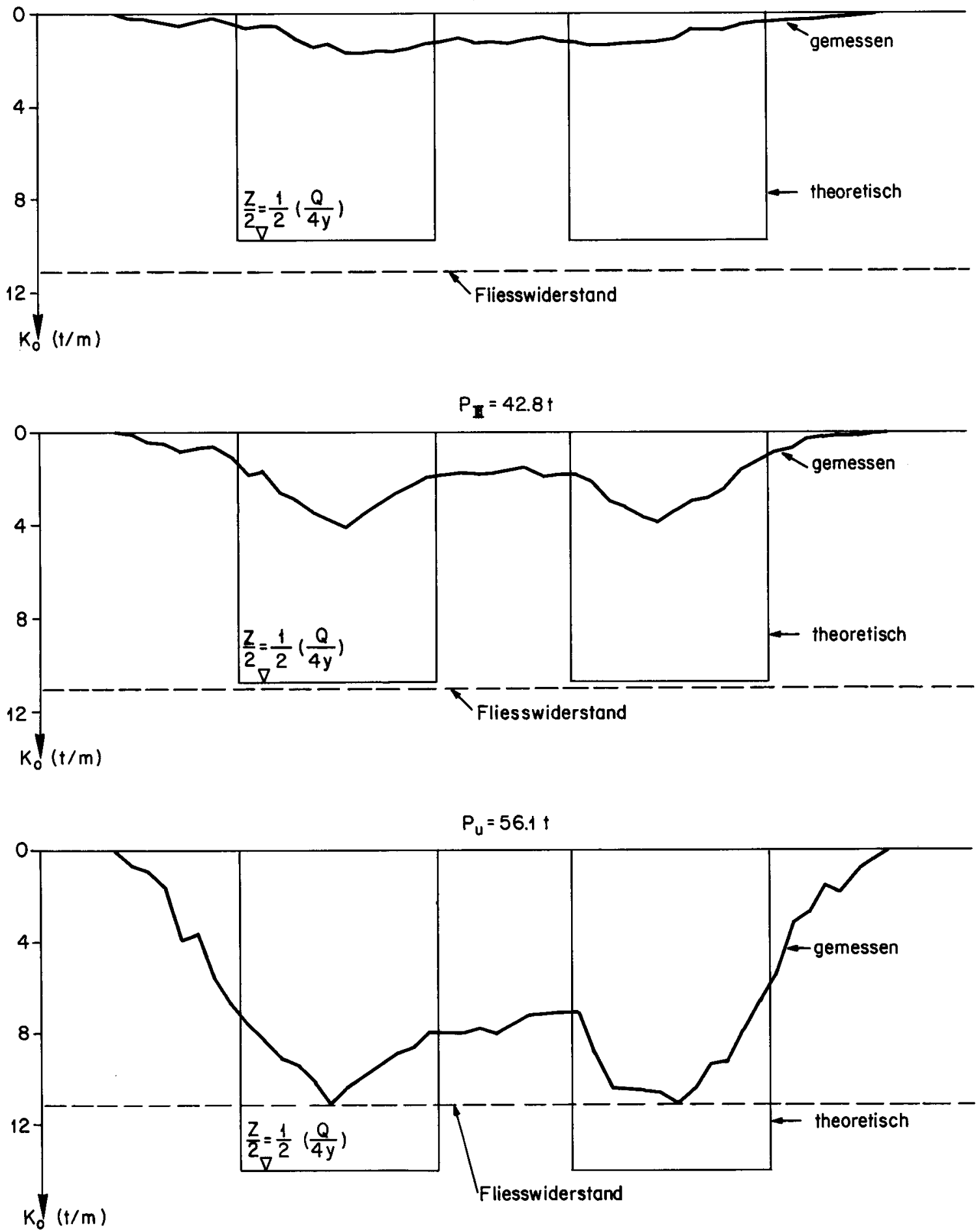

Bild 63: Theoretische und gemessene Zugkraft pro Längeneinheit in der oberen Lage der Plattenarmierung bei Balken $Q 2$ 


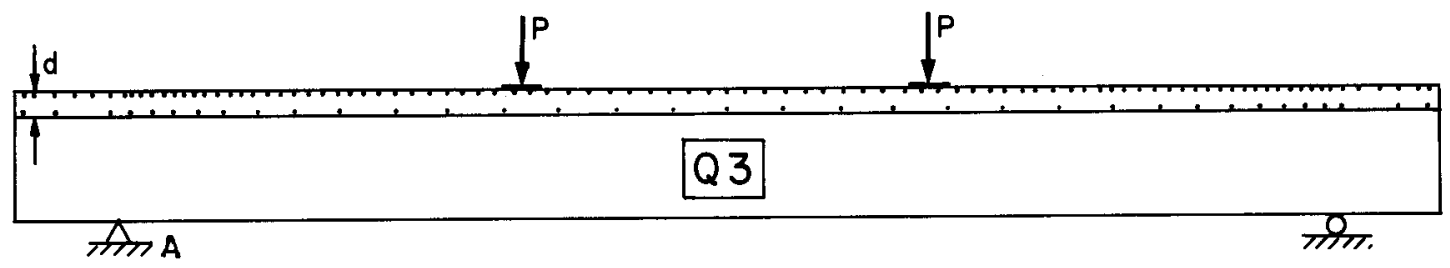

$\bar{P}_{I}=1.64 t, P_{I}=23.6 t$
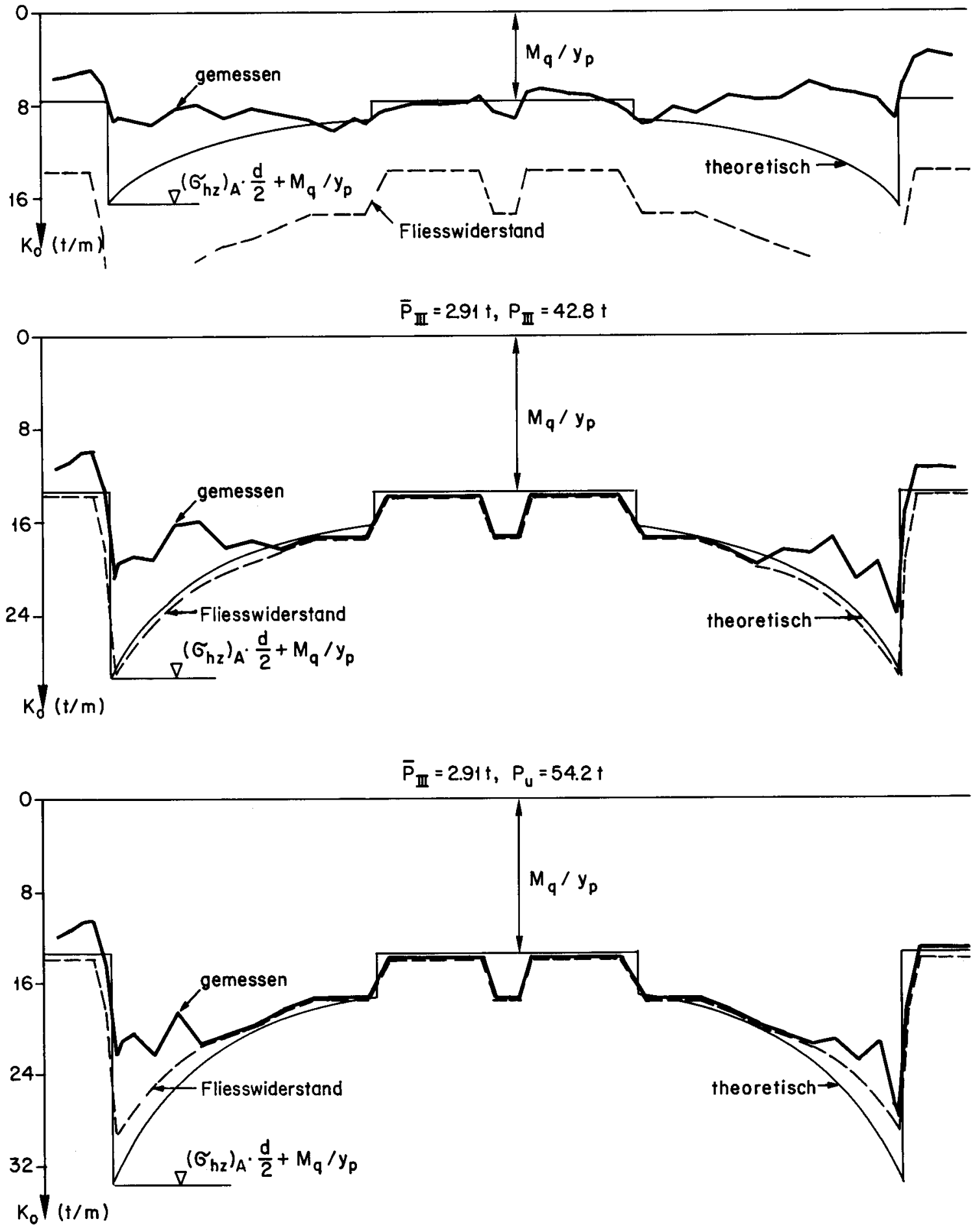

Bild 64: Theoretische und gemessene Zugkraft pro Längeneinheit in der oberen Lage der Plattenarmierung bei Balken Q 3 


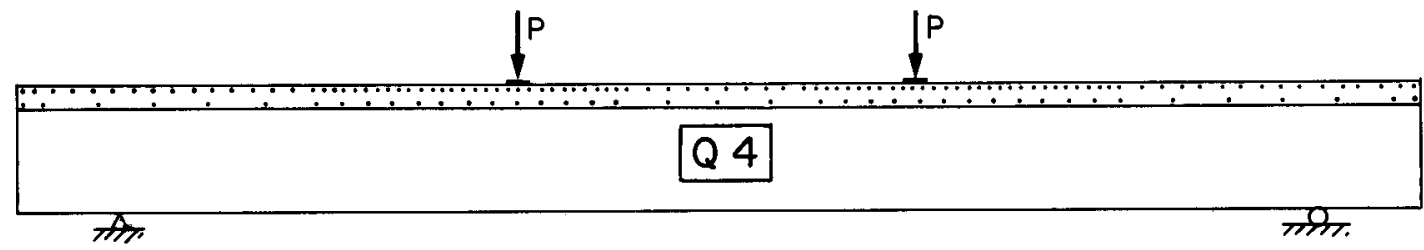

$\bar{P}_{I}=1.64 \uparrow, P_{I}=23.6 \dagger$

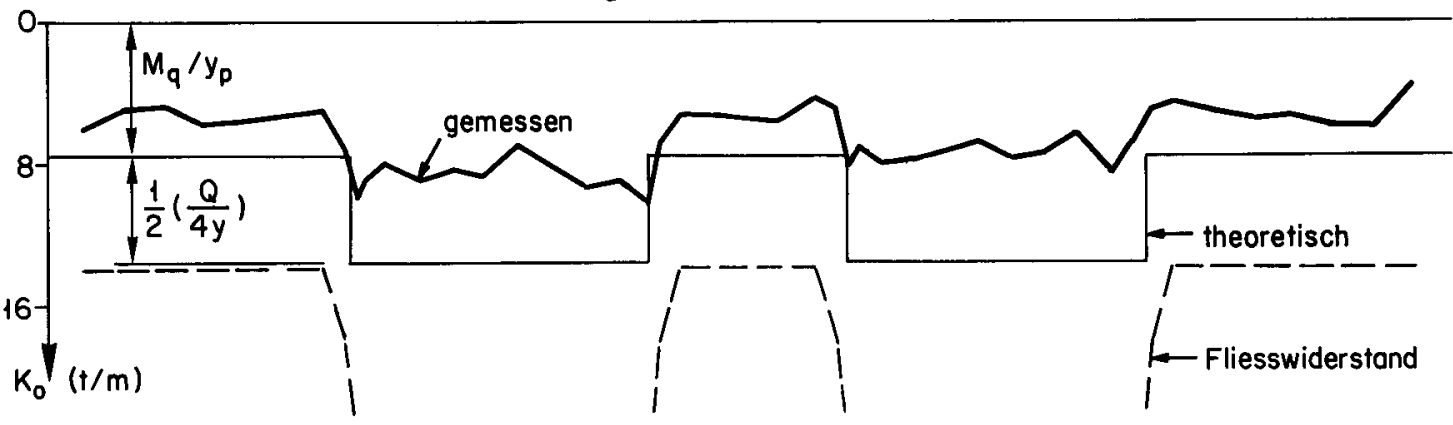

$\bar{P}_{\text {II }}=2.91 \dagger, P_{\text {III }}=42.8 \dagger$

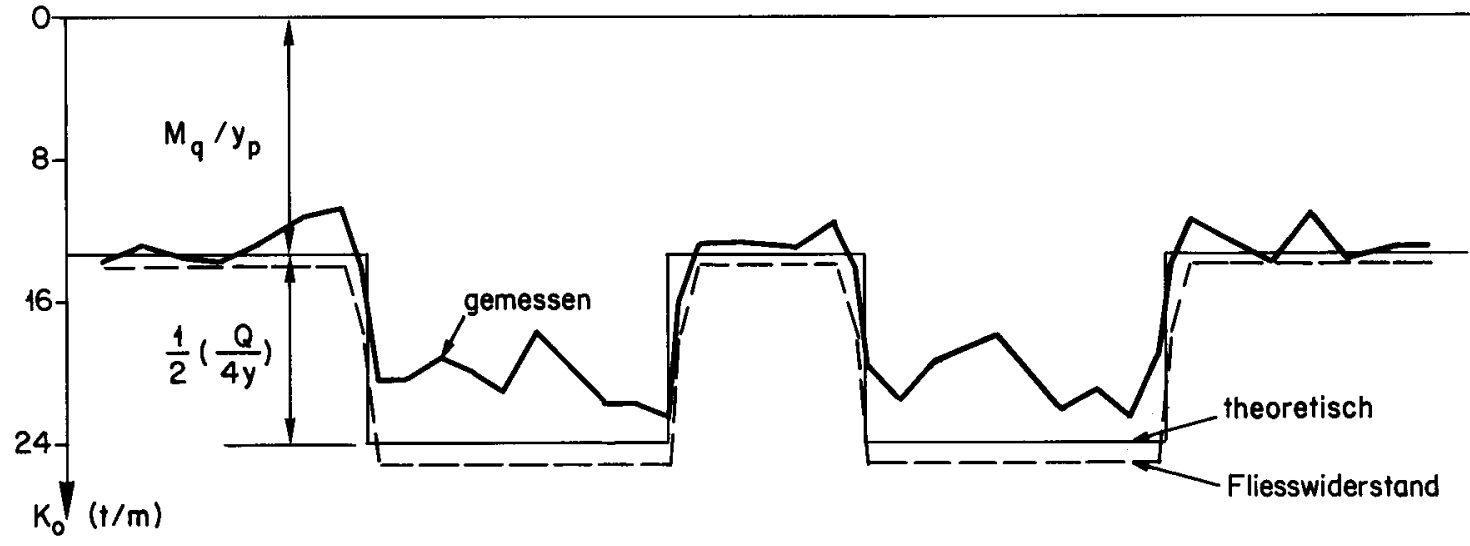

$\bar{P}_{\text {II }}=2.91 \uparrow, P_{u}=56.0 \dagger$

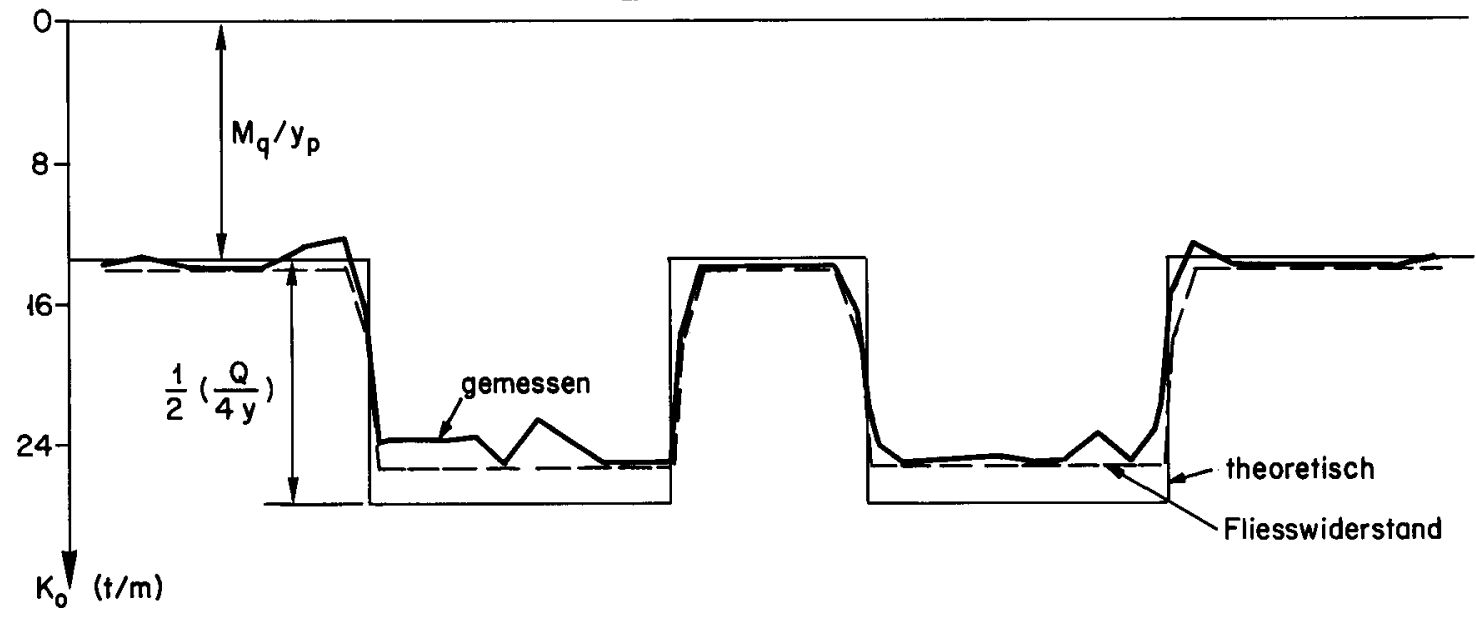

Bild 65: Theoretische und gemessene Zugkraft pro Längeneinheit in der oberen Lage der Plattenarmierung bei Balken Q 4 


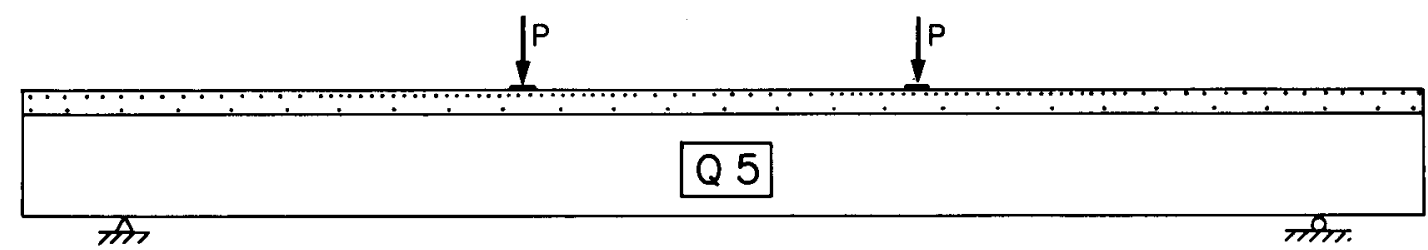

$\bar{P}_{I}=1.64 t, P_{I}=23.6 t$

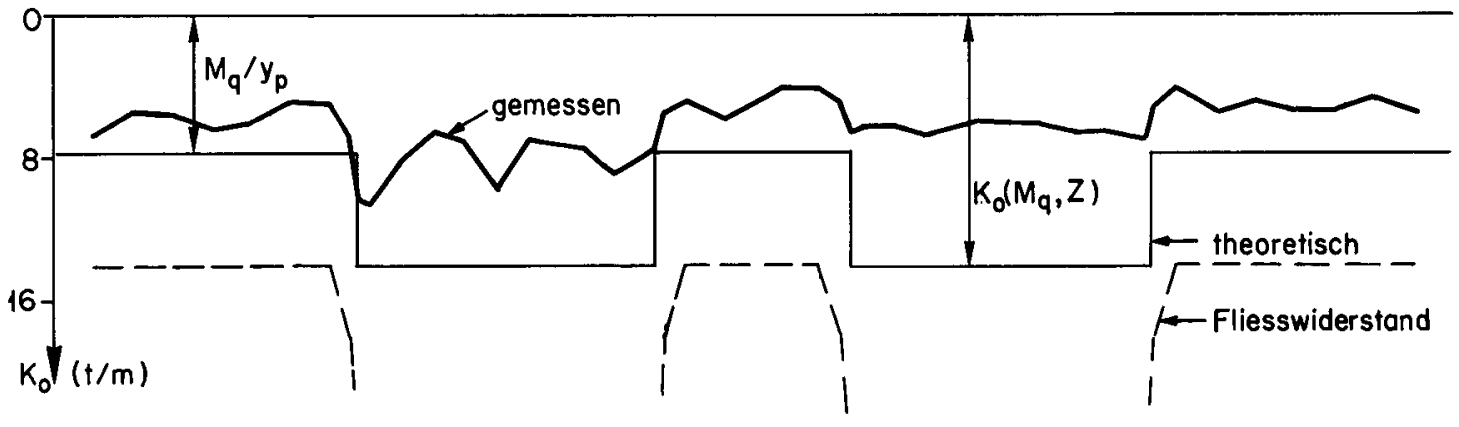

$\bar{P}_{\text {III }}=2.91+P_{\text {III }}=42.8 \dagger$

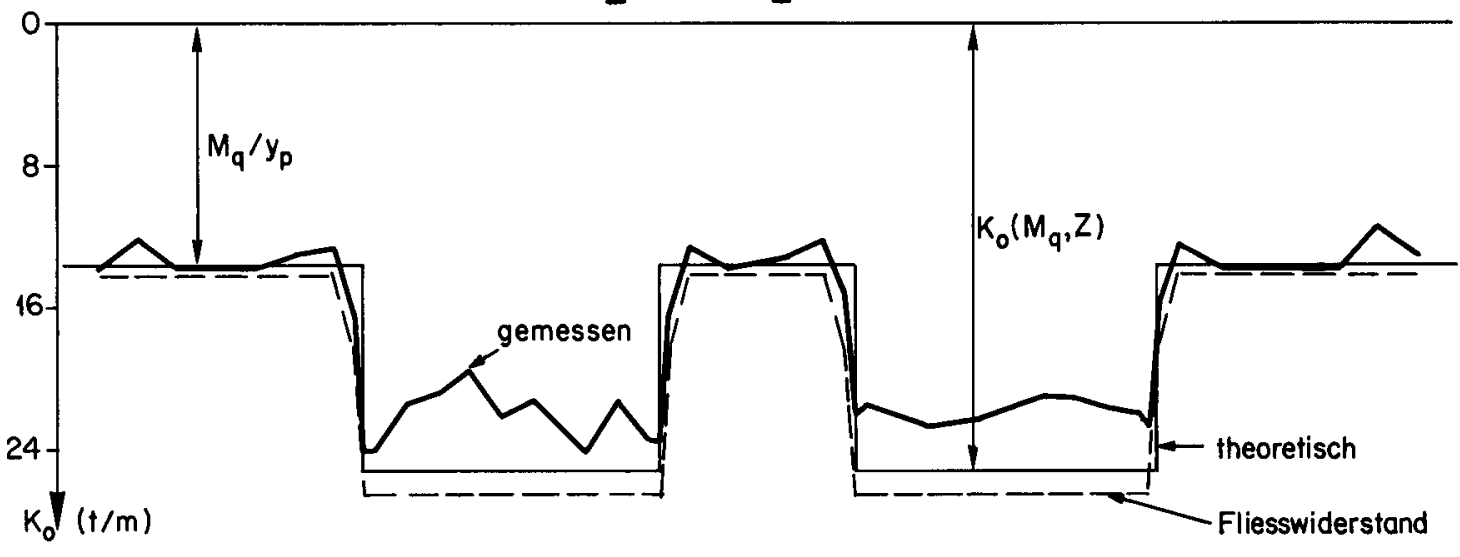

$\bar{P}_{\text {III }}=2.91 \mathrm{t}, P_{u}=52.3 \dagger$

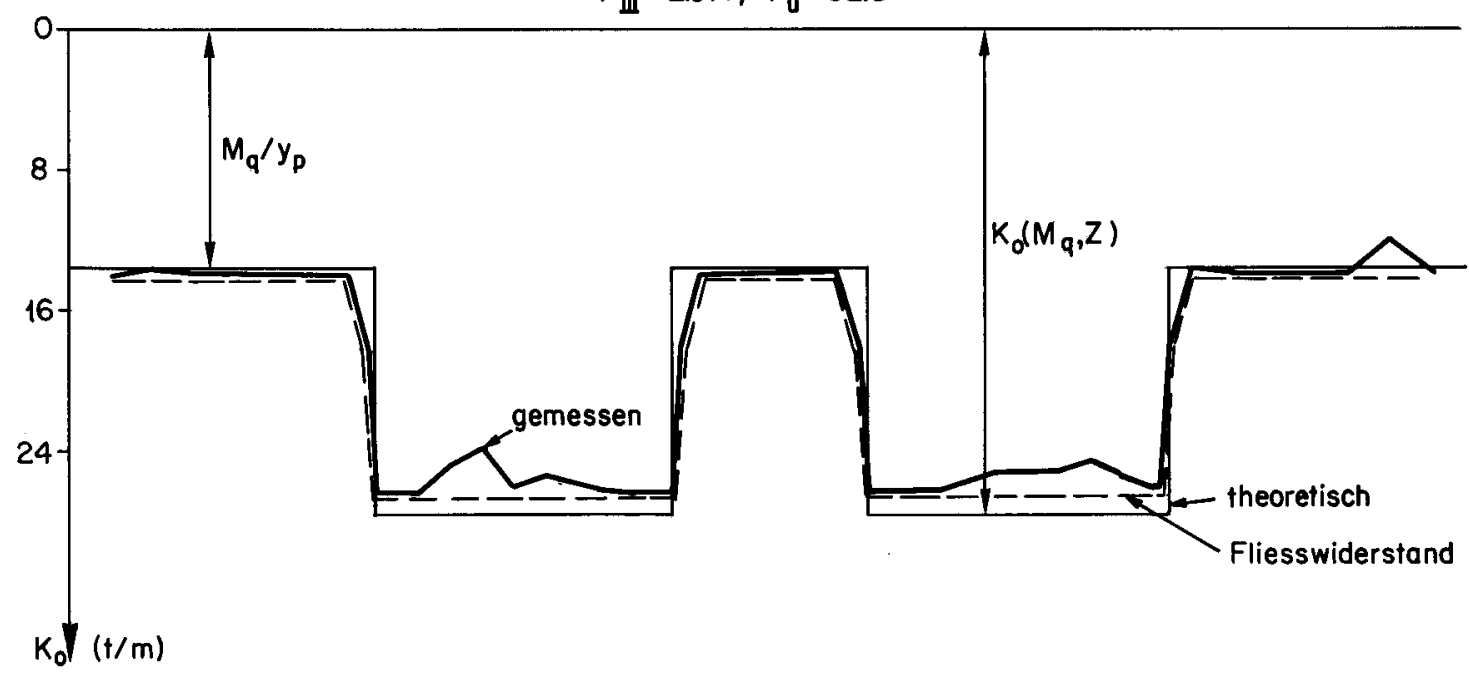

Bild 66 : Theoretische und gemessene Zugkraft pro Längeneinheit in der oberen Lage der Plattenarmierung bei Balken Q 5 
Versuche zur Bestimmung des Verbundkoeffizienten $\kappa$

Der Verlauf der Stahldehnung in einem Risselement ist in Bild 67 schematisch aufgezeichnet. Die Stahldehnung ist im Rissquerschnitt am grössten ( $\varepsilon_{\text {e max }}$ ), rechts und links davon nimmt sie infolge der durch das Ausziehen des Stahls aus dem Beton entstehenden Verbundspannungen ab. Bei der Ermittlung der Stahldehnungen mit Deformetern wird eine mittlere Dehnung $\varepsilon_{\text {em }}$ über die Messstrecke a gemessen, wobei

$$
\varepsilon_{e m}=\frac{1}{a} \int_{0}^{a} \varepsilon_{e}(x) \cdot d x
$$

Das Verhältnis

$$
\kappa=\frac{\varepsilon_{\text {em }}}{\varepsilon_{\text {e max }}}
$$

wird als Verbundkoeffizient bezeichnet. Dieser ist abhängig von zahlreichen Parametern, sa von Rissabstand, Stabdurchmesser, Höhe der Stahlbeanspruchung, Verlauf der Verbundspannungen längs der Zugarmierung, Form des $\sigma-\varepsilon-D i a g r a m m e s ~ d e s ~ S t a h l s$, Betoneigenschaften, usw.

Die in der Platte bei den Trägern Q1 bis Q5 gemessenen Dehnungen sind mittlere Dehnungen $\varepsilon_{e m}$. Die maximale Spannung im Stahl entspricht aber $\varepsilon_{e}$ max, und folglich benötigte man Angaben über den Verbundkoeffizienten $\kappa$. Zur Bestimmung dieses Koeffizienten wurden vier Nebenversuche 21 bis $Z 4$ durchgeführt: Jeweils zwei Stäbe aus dem Plattenarmierungsstahl $\oslash 6 \mathrm{~mm}$ wurden in einem Betonkörper $10 \times 10 \times 66 \mathrm{~cm}$ einbetoniert (Bild 68). Die Kärper $Z 1$ und $Z 2$ wiesen je einen einzigen vorgegebenen Riss auf, der durch Einbetonieren einer dünnen Metallscheibe erzeugt wurde. Bei Z1 lag dieser Riss in einem Querschnitt mit Messpunkten, bei $z 2$ in der Mitte zwischen den Messpunkten. Bei $Z 3$ und $Z 4$ wurden viele vorgegebene Risse im Abstand von $10 \mathrm{~cm}$ angeordnet. Bei Z3 fielen die vorgegebenen Risse mit den Querschnitten mit Messpunkten zusammen, bei Z4 lagen sie in der Mitte zwischen diesen Querschnitten.

Die Versuchskörper wurden in einer Zugmaschine zentrisch gezogen (Bild 69). An den oberen freien Strecken der Stahlstäbe wurde die Stahldehnung mit Hilfe von zwei elektronischen Dehnungsgebern gemessen. Der Mittelwert dieser zwei Dehnungen wurde als $\varepsilon_{e \text { max }}$ angesehen. Bei maximalen Dehnungen van 1.143\%0, $1.668 \% 0,1.991 \% 0$ und $2.185 \% 0$ wurden jeweils die mittleren Dehnungen entlang der vier Zugkörper auf übliche Weise gemessen (Bild 68). Im Fliessbereich (Dehnung bei Fliessbeginn $=2.34 \%$ ) wurden die Versuche nicht weitergeführt, da $k$ dort keinen Einfluss auf die Stahlspannung mehr hat.

Bei den Messungen konnte je nach Rissabstand und Lage der Risse in bezug auf die Messstrecke zwischen sechs Typen unterschieden werden (Bild 71). Bei Typ 1 (Rissabstand $=15 \mathrm{~cm}$ ) wurden die Messungen unmittelbar neben einem zufälligen Riss durchgeführt; bei Typ 2 (Rissabstand $=10 \mathrm{~cm}$ ) wurden sie über einem vorgegebenen Riss vorgenommen. Bei einem Rissabstand von $20 \mathrm{~cm}$ wurde zwischen Messungen neben zufälligen Rissen (Typ 3) und solchen neben vorgegebenen Rissen (Typ 3') differenziert. Typ 4 und Typ 4' 
weisen Rissabstände von $10 \mathrm{~cm}$ auf, wobei die Messstrecke bei Typ 4 mit einem zufälligen und einem vorgegebenen Riss abgegrenzt ist. Bei Typ 4' sind beide Risse vorgegeben.

Der Mittelwert des Verbundkoeffizienten $k$ ist für diese sechs Typen bei den verschiedenen $\varepsilon_{e} \max$ in Bild 71 angegeben. Aus dem Vergleich zwischen Typ 3 und Typ 3' sowie Typ 4 und Typ 4' sieht man, dass $k$ bei vorgegebenen Rissen höher liegt als bei zufällig entstandenen Rissen.

Die Uebertragung der gewonnenen $\kappa$-Werte auf die Platte bei den Balkenversuchen ist sehr schwierig. Immerhin kann man aus den Rissebildern der Platten ablesen, dass bei den Balken Q3, Q4 und Q5 die Längsrisse in der Platte im allgemeinen durch die Messpunkte verlaufen (z.B. Bild 51). Da die Risse in der Platte zufällig entstehen, sind sie am ehesten vergleichbar mit den Rissen bei Typ 4: Rissabstand $10 \mathrm{~cm}$ und Risse bej den Messpunkten. Für diesen Fall liegt $\kappa$ zwischen 0.84 und 0.88 .

Bei den Balken $Q 1$ und Q2 treten entweder mehrere Risse innerhalb der Messstrecke auf (Bild 43), oder es grenzen zwei Risse die Messstrecke ab, also wiederum etwa wie bei Typ 4 .

Bei den Platten der Versuchsträger haben indessen zwei zusätzliche Aspekte auch noch einen Einfluss auf den Wert von k: Erstens ist eine Druckspannung aus der Längsbiegung vorhanden, welche die Verbundeigenschaften zwischen Beton und Stahl verbessert; somit wird $k$ bei der Platte kleiner als bei Typ 4. Dieser Effekt dürfte allerdings nicht wesentlich sein. Andererseits entstehen mit zunehmender Belastung weitere Risse innerhalb der Messstrecken, so dass $k$ grösser wird.

Aufgrund der Resultate dieser Nebenversuche und der angeführten Erwägungen wurde zur Abschätzung der maximalen Dehnungen und damit zur Ermittlung der Spannungen und Kräfte in der Plattenarmierung bei allen fünf Balken Q1 bis Q5 ein $k$-Wert von 0.9 verwendet. 

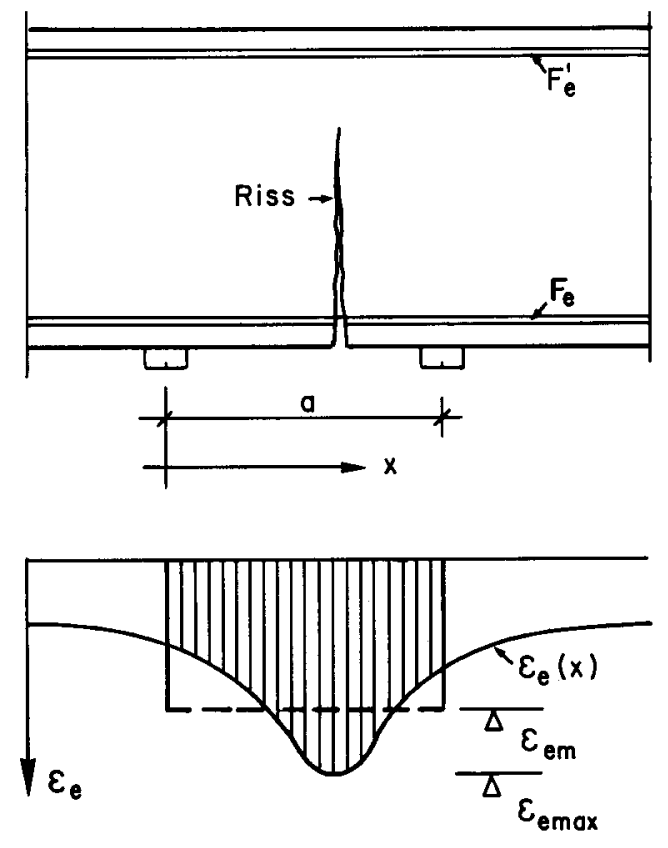

$\varepsilon_{e m}=\frac{1}{0} \int_{0}^{0} \varepsilon_{e}(x) \cdot d x$

$\kappa=\frac{\varepsilon_{\mathrm{em}}}{\varepsilon_{\mathrm{e} \text { max }}}$

Bild 67 : Risselement
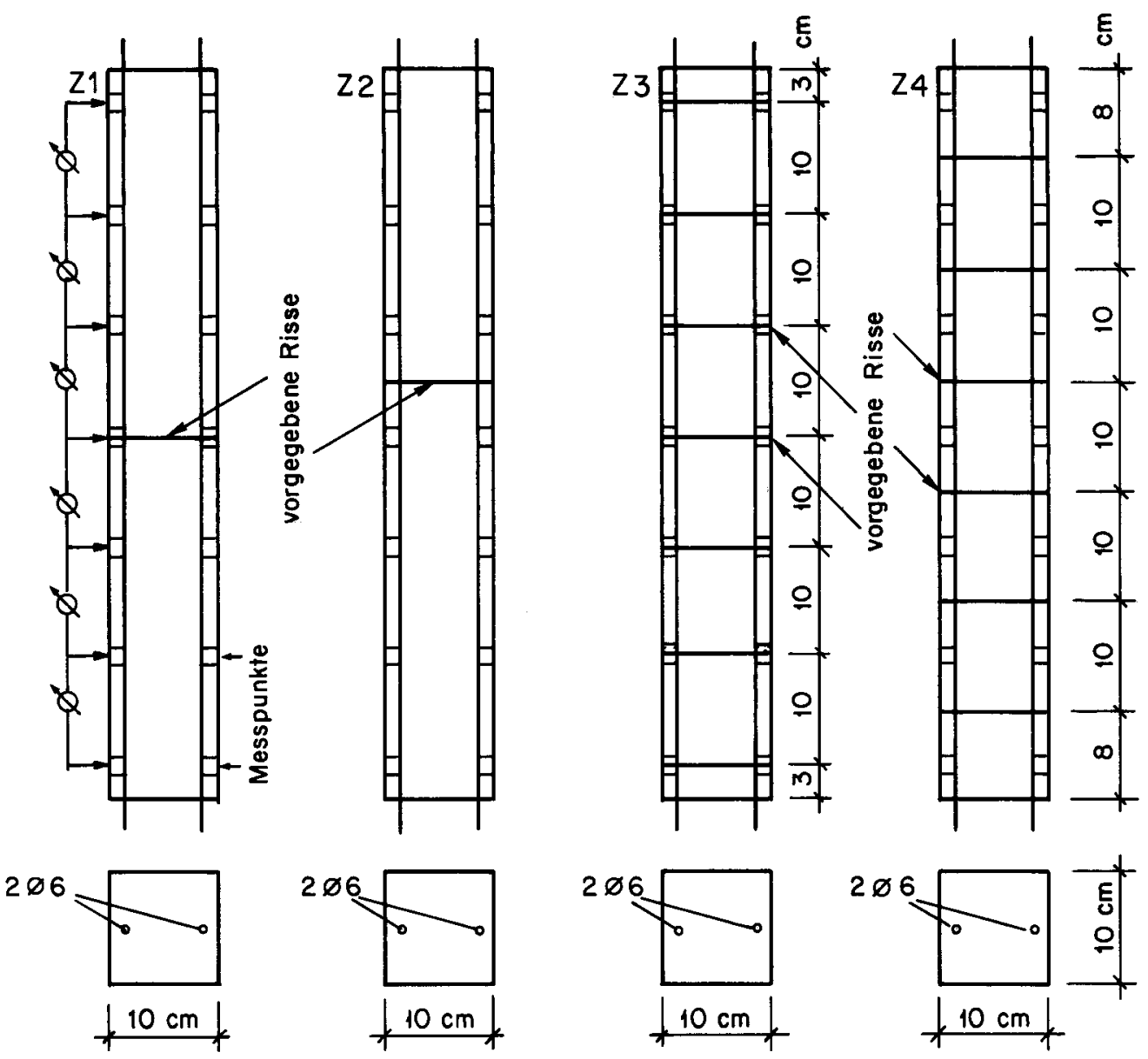

Bild 68: Versuchskörper zur Bestimmung von $k$ 


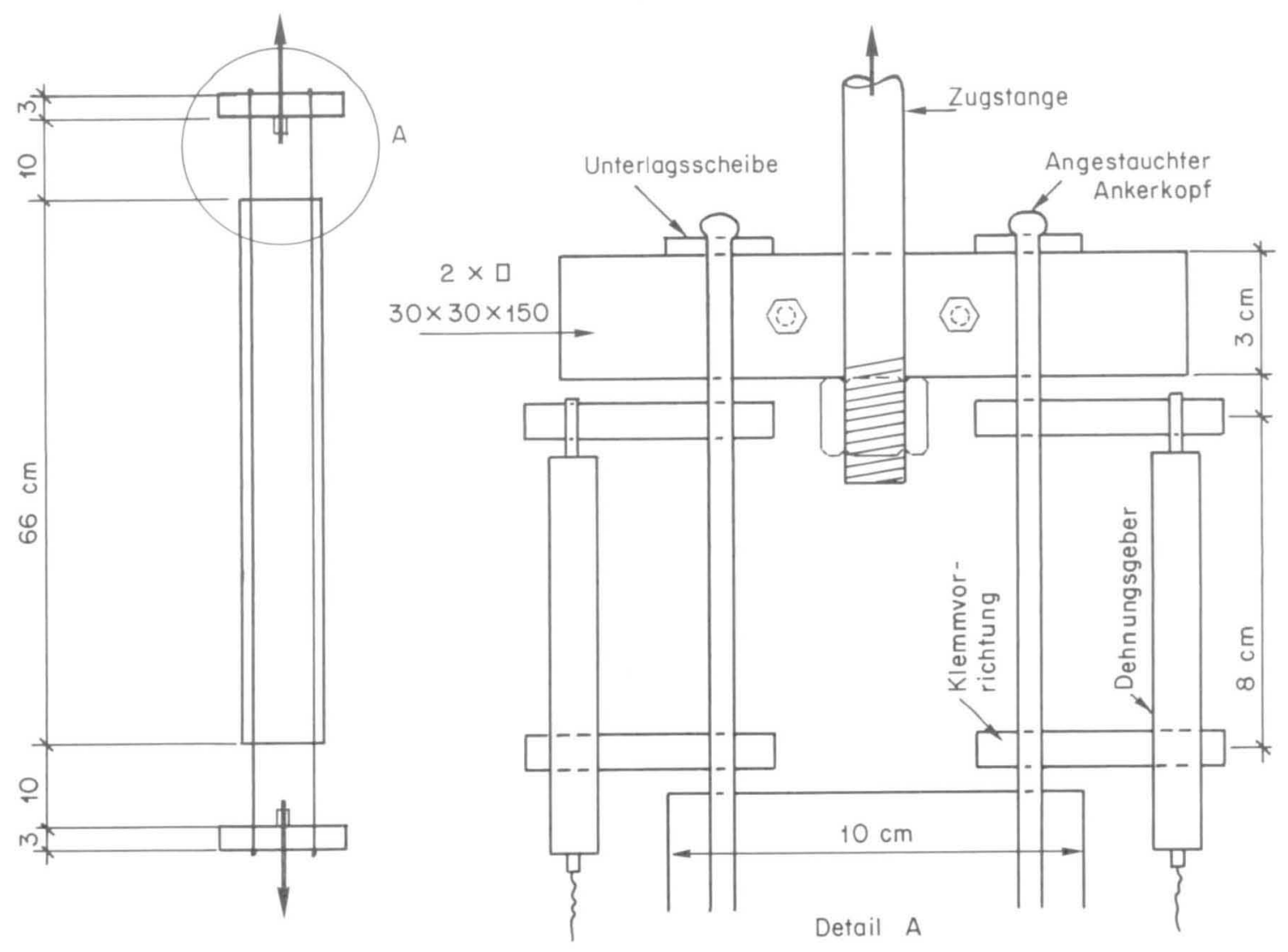

Bild 69: Zug-und Messvorrichtungen
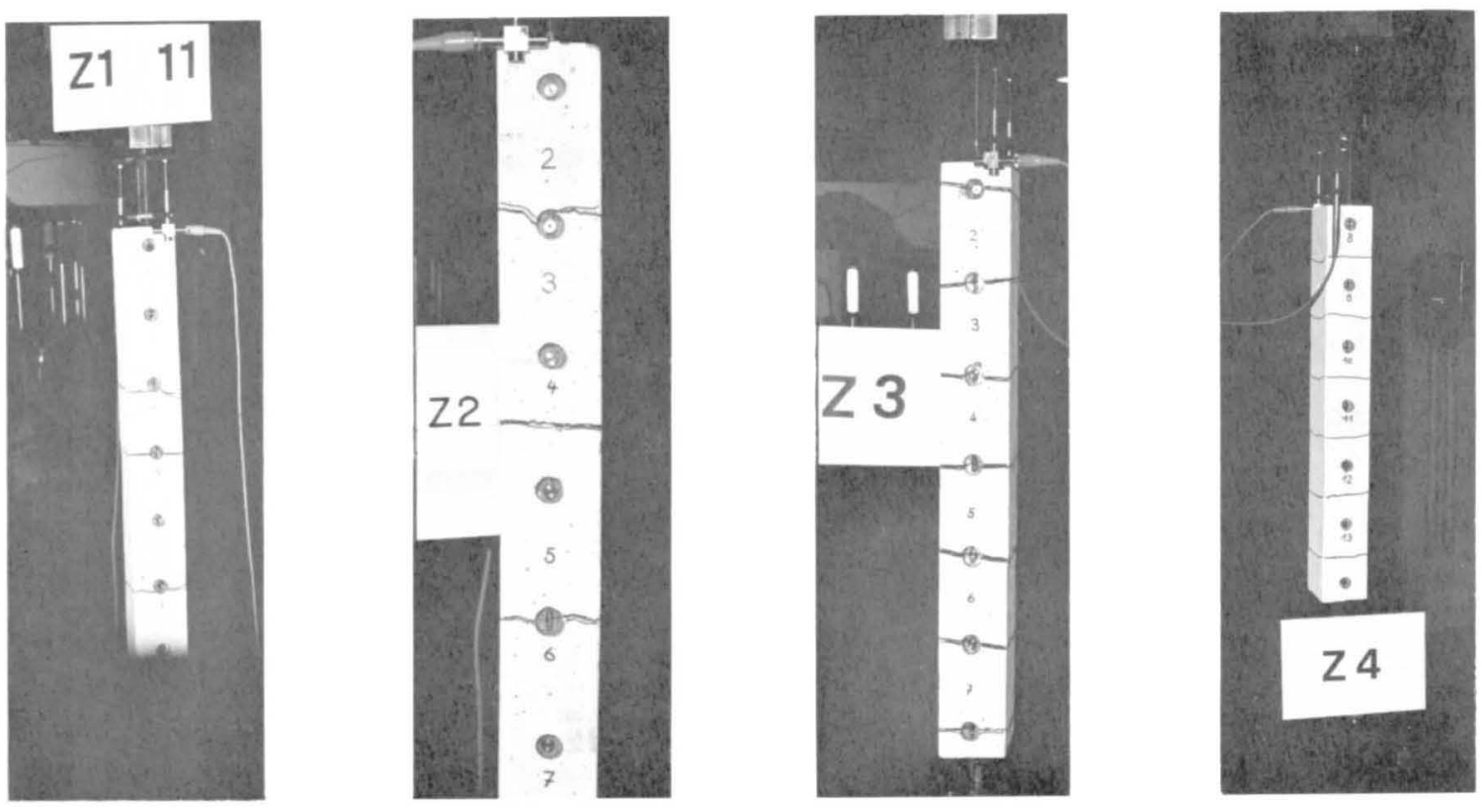

Bild 70: Versuchskörper nach dem Prüfen 


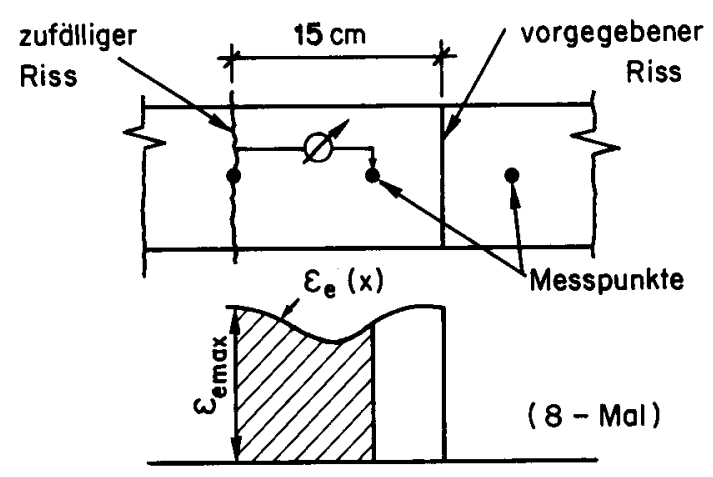

Typ 1

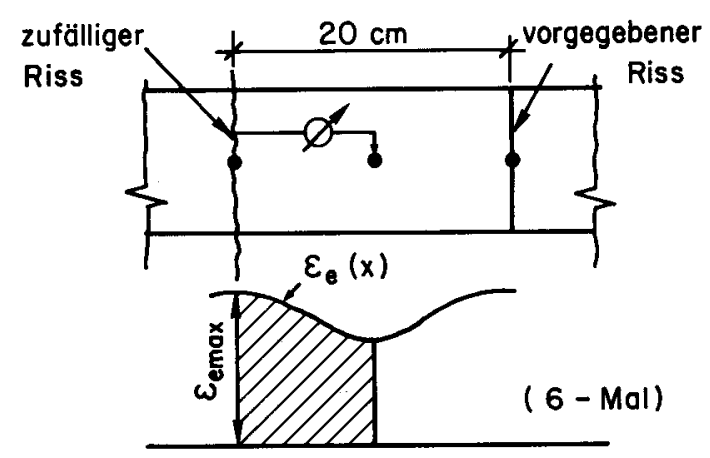

$\underline{\text { Typ } 3}$

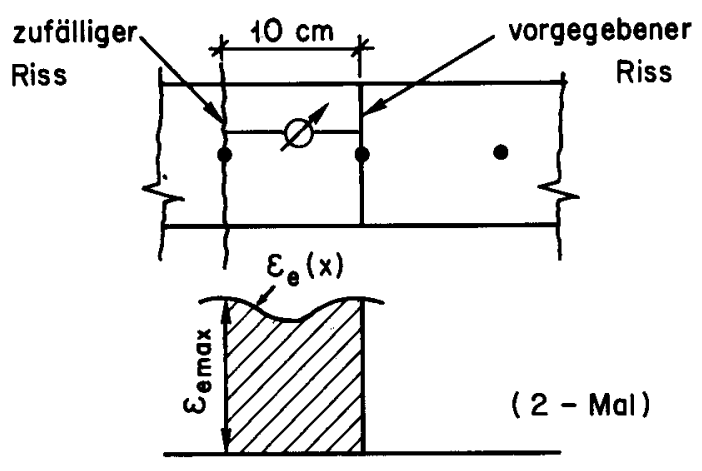

Typ 4

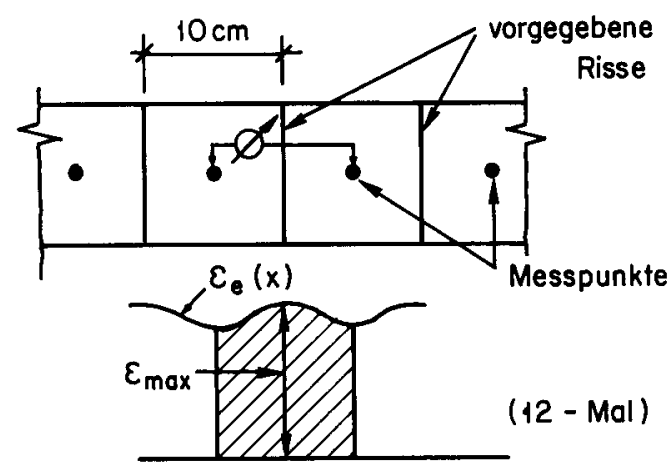

Typ 2

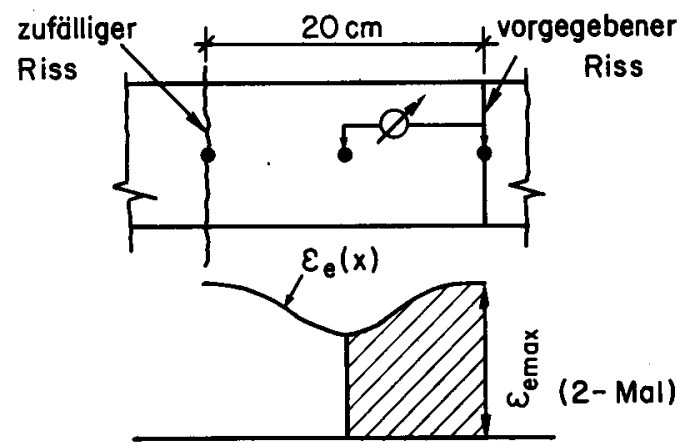

Typ 3'

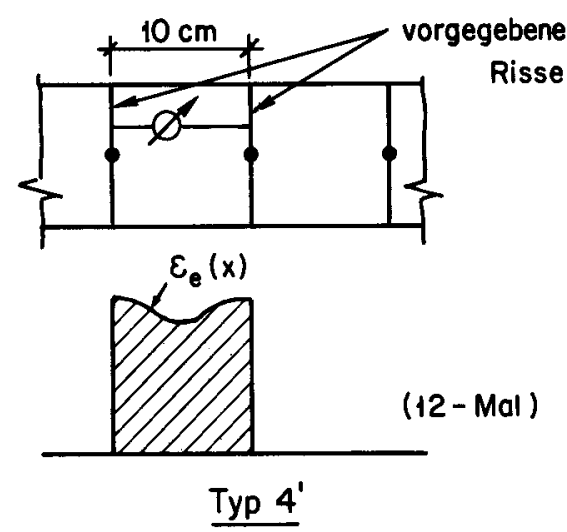

\begin{tabular}{|c|c|c|c|c|c|c|c|}
\hline \multirow{2}{*}{$\begin{array}{c}\varepsilon_{\text {emax }} \\
\% \circ\end{array}$} & $\begin{array}{c}\sigma_{e} \\
t / \mathrm{cm}^{2}\end{array}$ & \multicolumn{6}{|c|}{ Verbundkoeffizient $\kappa$} \\
\cline { 3 - 8 } & & Typ 1 & Typ 2 & Typ 3 & Typ 3' & Typ 4 & Typ 4' \\
\hline 1,143 & 2,400 & - & 0,86 & - & - & - & 0,87 \\
1,668 & 3,50 & 0,86 & 0,91 & 0,67 & 0,79 & 0,84 & 0,93 \\
1,991 & 4,18 & 0,85 & 0,92 & 0,73 & 0,84 & 0,88 & 0,94 \\
2,185 & 4,59 & 0,89 & 0,91 & 0,74 & 0,90 & 0,85 & 0,96 \\
\hline
\end{tabular}

Bild 71: Auswertung der $\kappa$-Versuche 